\title{
Elektromagnetische Arraymessungen im Rheinischen Schiefergebirge: Modelle der elektrischen Leitfähigkeit der Erdkruste und des oberen Mantels mit Verbindungen zum Eifelvulkanismus
}

\author{
Dissertation \\ zur Erlangung des Doktorgrades \\ der Mathematisch-Naturwissenschaftlichen Fakultäten \\ der Georg-August-Universität zu Göttingen
}

\author{
vorgelegt von \\ Jörg Leibecker \\ aus Osnabrück
}

Göttingen 2000 
D7

Referent:

Prof. Dr. Karsten Bahr

Korreferent:

Prof. Dr. Ulrich Christensen

Tag der mündlichen Prüfung:

20.04.2000 
In den Wissenschaften ist viel Gewisses, sobald man sich von den Ausnahmen nicht irremachen läßt und die Probleme zu ehren weiß.

Johann Wolfgang von Goethe 


\section{Inhaltsverzeichnis}

$\begin{array}{ll}\text { Einleitung } & 1\end{array}$

1 Grundlagen der elektromagnetischen Tiefenforschung $\quad 4$

1.1 Das anregende Feld . . . . . . . . . . . . . . . . . . . . 4

1.2 Physikalische Grundlagen . . . . . . . . . . . . . . 5

1.3 Herleitung und Skalierung der Helmholtzgleichung . . . . . . . . . . . 7

1.4 Verhalten der Feldgrößen an Grenzflächen . . . . . . . . . . . . . . . 9

1.5 Induktion im homogenen Halbraum . . . . . . . . . . . . . . . 10

1.6 Magnetotellurik . . . . . . . . . . . . . . . . . . . . 14

1.7 Erdmagnetische Tiefensondierung . . . . . . . . . . . . . . . 16

1.8 Verzerrung elektromagnetischer Felder durch lokale Störkörper . . . . . . 20

2 Strukturbestimmungen $\quad \mathbf{2 4}$

2.1 Modellvorstellungen in der elektromagnetischen Tiefenforschung . . . . 25

2.1.1 Die Nebendiagonalform des Impedanztensors . . . . . . . . . . . 25

2.1.2 Anisotropie der Leitfähigkeit . . . . . . . . . . . . . . . . . . 26

2.1.3 Regionale Induktion und galvanische Verzerrung . . . . . . . . . 28

2.2 Rotationsinvariante Maßzahlen . . . . . . . . . . . . . . . . . . 29

2.2.1 Anwendungen in der Magnetotellurik . . . . . . . . . . . . . 31

2.2.2 Anwendungen in der erdmagnetischen Tiefensondierung . . . . . 34

2.3 Rotationswinkel . . . . . . . . . . . . . . . . . . . 38

2.3.1 Behandlung des Impedanztensors . . . . . . . . . . . . . . . . . . . . . . . . . . 48

2.3.2 Behandlung des Perturbationstensors . . . . . . . . . . . . 40

2.4 Beiträge zur Analyse des Perturbationstensors . . . . . . . . . . . . . . 40

2.4.1 Zur Wahl der Referenzstation . . . . . . . . . . . . . . 40

2.4.2 Vorschlag zur Simulation des magnetischen Normalfeldes . . . . 44

2.4.3 Einfluß der Verzerrung auf das magnetische Horizontalfeld . . . . 45

3 Die Feldmessungen $\quad 49$

3.1 Zur Geologie des Rheinischen Schiefergebirges . . . . . . . . . . . . . 49

3.1.1 Überblick über die geologische Entwicklung . . . . . . . . . . . 49

3.1.2 Die Hebung des Rheinischen Schildes . . . . . . . . . . . . . . . . 50

3.1.3 Das europäische Riftsystem . . . . . . . . . . . . 51 
3.1.4 Quartärer Vulkanismus in der Eifel . . . . . . . . . . . . . . 52

3.2 Die Meßkampagne . . . . . . . . . . . . . . . . . . . 54

3.2.1 Zur Auswahl der Stationsstandorte . . . . . . . . . . . . . . 54

3.2 .2 Die Meßapparatur . . . . . . . . . . . . . . . 55

3.2.3 Durchführung der Feldmessungen . . . . . . . . . . . . . . . . 56

3.2.4 Aufzeichnung elektromagnetischer Zeitreihen . . . . . . . . . . . 59

3.3 Datenanalyse . . . . . . . . . . . . . . . . 60

3.3.1 Vorbereitung der Rohdaten . . . . . . . . . . . . . . . . . 60

3.3.2 Berechnung von geglätteten Spektren . . . . . . . . . . . . . 61

3.3.3 Schätzung der Übertragungsfunktionen . . . . . . . . . . . . . 62

4 Ergebnisse der Datenanalyse $\quad 64$

4.1 Überblick . . . . . . . . . . . . . . . . . . . . . . . . 64

$4.1 .1 \rho^{*}\left(z^{*}\right)$-Darstellung . . . . . . . . . . . . . . 64

4.1.2 Phase der Impedanz und scheinbarer spezifischer Widerstand . . . 66

4.1.3 Bestimmung von Vorzugsrichtungen . . . . . . . . . . . . . 69

4.2 Daten zur Kruste . . . . . . . . . . . . . . . . . . . 72

4.2.1 Rotationsinvariante Maßzahlen des Perturbationstensors . . . . . 72

4.2.2 Magnetische Übertragungsfunktionen . . . . . . . . . . . . . . . 74

4.2.3 Lokale Induktionspfeile und Perturbationspfeile bei $256 \mathrm{~s}$. . . . 78

4.2.4 $\rho^{*}\left(z^{*}\right)$-Darstellung und Phasenaufspaltung bei kurzen Perioden . 81

4.3 Daten zum oberen Mantel . . . . . . . . . . . . . . . . . 84

4.3.1 Phasenaufspaltung bei langen Perioden . . . . . . . . . . . 84

4.3.2 Analyse des Perturbationstensors . . . . . . . . . . . . . . . 89

4.3 .3 Lokale Induktionspfeile . . . . . . . . . . . . . . . . . . . 89

4.4 Zusammenstellung der wichtigsten Resultate . . . . . . . . . . . . . 92

5 Interpretation der Daten durch dreidimensionale Modelle $\quad 93$

5.1 Zur Modellentwicklung . . . . . . . . . . . . . . . . . . . . . . 93

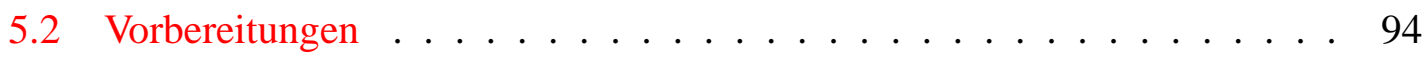

5.2.1 Sichtung der Daten . . . . . . . . . . . . . . . . . 94

5.2.2 Das Hintergrundmodell . . . . . . . . . . . . . . . . . 95

5.2.3 Zur Modellierung einer anisotropen Schicht . . . . . . . . . . . . . . . . 96

5.2.4 Diskretisierung des 3D-Modellkernes . . . . . . . . . . . . 100

5.3 Ein Modell der Leitfähigkeitsverteilung unter der Eifel . . . . . . . . . . 101

5.4 Die Modellantwort . . . . . . . . . . . . . . . . . . . . . . . 104

5.4.1 Die Anomalie im magnetischen Feld . . . . . . . . . . . . . . . . 104

5.4.2 Die Anpassung der Phase der Impedanz . . . . . . . . . . . . . . 107

5.5 Ein lokales Modell der Leitfähigkeitsverteilung bei Station DIE . . . . . . 111

5.6 Flächenhafte Verteilung der Induktionspfeile bei langen Perioden . . . . . 115

$\begin{array}{ll}\text { Diskussion } & 118\end{array}$ 
$\begin{array}{ll}\text { Literaturverzeichnis } & 125\end{array}$

$\begin{array}{lr}\text { A Anhang } & \mathbf{1 3 5}\end{array}$

A.1 Stationskoordinaten . . . . . . . . . . . . . . . . 135

A.2 Elemente des Perturbationstensors nach Wechsel der Bezugsstation . . . . 136

A.3 Nachträge zum Prozessing . . . . . . . . . . . . . . . . 136

A.3.1 Gewichtsfunktionen . . . . . . . . . . . . 136

A.3.2 Explizite Ausdrücke für alle Übertragungsfunktionen . . . . . . . 137

A.3.3 Gewichtete Stapelung der geglätteten Spektren . . . . . . . . . . 138

A.4 Göttinger Datenformate . . . . . . . . . . . . . . . . . . . 139

A.4.1 *.mtt-Format . . . . . . . . . . . . . 139

A.4.2 *.gdt-Format . . . . . . . . . . . . 139

A.5 Verwendete Programme . . . . . . . . . . . . . . . . . . . 140

A.5.1 Datenerfassung und Prozessing . . . . . . . . . . . . 140

A.5.2 Berechnung der Darstellungsgrößen mit emdisp . . . . . . . . . 140 


\section{Einleitung}

„Die Eyffel hat ihres Gleichen in der Welt nicht; ... und ihre Kenntnis kann gar nicht umgangen werden, wenn man eine klare Ansicht der vulcanischen Erscheinungen auf Continenten erhalten will.“

Dies schrieb der deutsche Geologe Leopold von Buch in einem Brief an Johann Steininger im Jahre 1820. Die Eifel, im westlichen Rheinischen Schiefergebirge zwischen Rhein und Ardennen gelegen, hat seit dieser Zeit tatsächlich eine bedeutende Rolle in der geologischen und paläontologischen Forschung gespielt.

Neben der Bedeutung als wichtige Fundstelle für Fossilien lag der zweite geowissenschaftliche Schwerpunkt bei vulkanologischen Fragestellungen. Die berühmtesten Vulkanformen der Eifel, die Maare, gelten weltweit als Musterbeispiele dieses Typs. Einige vulkanische Bildungen sind mit einem Alter von rund 10.000 Jahren die jüngsten in Mitteleuropa.

Es gibt derzeit weltweit ca. 500 - 600 aktive Vulkane ${ }^{1}$. Etwa $95 \%$ davon befinden sich in der Nähe von konvergierenden oder divergierenden Plattengrenzen. Nur rund 5\% befinden sich abseits der Ränder auf einer Platte. Die Vulkane der Eifel gehören zu letzterer Gruppe, sie liegen auf der Eurasischen Kontinentalplatte. Man spricht hier von einem Intraplattenvulkanismus. Während der Vulkanismus an den mittelozeanischen Rücken und an konvergierenden Plattengrenzen durch die Theorie der Plattentektonik recht gut erklärt werden konnte, stellte er weit von Plattengrenzen entfernt auftretend zunächst ein Problem dar.

Einen Erklärungsansatz bietet die Vorstellung von den sogenannten Mantelplumes ${ }^{2}$ (WILsON, 1963; MORGAN, 1972). Man versteht darunter heißes Material, das in einem relativ eng begrenzten Strahl $^{3}$ im Erdmantel aufsteigt. Diese vertikalen, zylinderförmigen Strömungen sind wahrscheinlich ortsfest im Mantel, so daß sich auf einer Lithosphärenplatte, die sich über den Mantelplume hinwegbewegt, eine Spur von zunehmend älteren Vulkanen bilden kann. Das bekannteste Beispiel dafür findet man in der Inselkette, zur der Hawaii gehört.

Ein solcher Mantelplume wird von DUNCAN et al. (1972) als Erklärung für die Vulkan-

\footnotetext{
${ }^{1}$ Die Vulkane auf dem Ozeanboden sind dabei nicht mitgezählt.

${ }^{2}$ Der Begriff Manteldiapir wird im folgenden synonym verwendet.

${ }^{3}$ Der Durchmesser liegt in einer Größenordnung von etwa 100 km.
} 
gebiete in Zentraleuropa vorgeschlagen.

Wahrscheinlich eng mit dem Vulkanismus verbunden ist die bis heute andauernde Hebung des Rheinischen Schildes um bis zu $1.6 \mathrm{~mm}$ pro Jahr (MÄLZER, 1983). Im Rahmen des Schwerpunktprogramms der Deutschen Forschungsgemeinschaft ,Vertikalbewegung und ihre Ursachen am Beispiel des Rheinischen Schildes“ (1976 - 1982) wurde jenes Phänomen intensiv untersucht. In diesem Zusammenhang wurde auch die Frage nach einem Mantelplume als mögliche Ursache erneut erörtert (NEUGEBAUER et al., 1983). Mehrere Untersuchungen zeigten nun allerdings, daß die zentraleuropäischen Vulkangebiete keine eindeutige Altersprogression aufweisen, wie man es bei dem Modell eines stationären Mantelplumes unter einer sich bewegenden Lithosphärenplatte erwarten würde (LIPPOLT, 1983).

Mit Hilfe der seismischen Tomographie fanden RAIKES \& BONJER (1983) eine Zone verringerter seismischer Kompressionswellengeschwindigkeit unter dem westlichen Rheinischen Schiefergebirge in einer Tiefe von $50-200 \mathrm{~km}$. Die Autoren vermuten dort einen Bereich im Mantel, der partiell aufgeschmolzen ist.

Großräumige geochemische Untersuchungen aus jüngerer Zeit von magmatischen Gesteinen aus Zentral- und Westeuropa sowie einigen Inseln im östlichen Atlantik zeigen, daß möglicherweise ein gemeinsames Reservoir in der Asthenosphäre existiert, aus der diese Vulkangebiete gespeist werden (HOERNLE et al., 1995). Ein tiefer Mantelplume ist zur Erklärung der Daten nicht erforderlich, wird aber auch nicht ausgeschlossen (WILSON \& Downes, 1992). Als Quelle kommt statt dessen möglicherweise eine großräumige Aufströmumg im oberen Erdmantel in Betracht, die von HoERnLE et al. (1995) mittels seismischer Tomographie nachgewiesen wurde. In einer Tiefe von $100 \mathrm{~km}$ erstreckt sie sich über eine Fläche von $2500 \mathrm{~km} \times 4000 \mathrm{~km}$ bis nach Nordafrika. Sie taucht in Richtung Südwesten ab und kann noch in Tiefen von etwa $500 \mathrm{~km}$ nachgewiesen werden. Das Auflösungsvermögen dieser Untersuchungen reicht jedoch nicht aus, um lokale Anomalien unter den Vulkangebieten zu erkennen.

Im Rahmen eines kleinräumigen teleseismischen Experiments mit entsprechend höherer Auflösung zeigten GRANET et al. (1995), daß unter dem französischen Massif Central wahrscheinlich ein Mantelpume existiert. Sie vermuten auch unter mehreren anderen variszischen Gebirgen Zentral- und Westeuropas kleine Manteldiapire, deren Quelle die von HOERNLE et al. beschriebene Schicht sein könnte.

In diesem Zusammenhang geriet auch die Frage nach einem Mantelplume unter dem Rheinischen Schiefergebirge wieder in das Zentrum des Interesses. Es wurden umfangreiche Projekte gestartet, an denen verschiedene geowissenschaftliche Disziplinen beteiligt waren. Im Rheinischen Schiefergebirge und in dessen Umgebung wurden mehrere großangelegte Feldexperimente durchgeführt. Dazu gehörten unter anderem seismologische Messungen (RITTER et. al, 2000) und ein elektromagnetisches Experiment, dessen Ergebnisse in der vorliegenden Arbeit vorgestellt werden.

Bei den hier verwendeten Verfahren werden die natürlichen Variationen des Erdmagnetfeldes ausgenutzt, um durch Messung der induzierten elektrischen und magnetischen Felder an der Erdoberfläche die räumliche Leitfähigkeitsverteilung im Untergrund zu bestimmen. Geht man davon aus, daß ein Mantelplume partielle Schmelzen enthält, die 
gegenüber dem umgebenden Material eine erhöhte elektrische Leitfähigkeit aufweisen, so sollten die Methoden der elektromagnetischen Tiefenforschung ein geeignetes Mittel darstellen, um weitere Beiträge zur Klärung der Plumehypothese liefern zu können.

Im Rheinischen Schiefergebirge wurden in der Vergangenheit bereits mehrfach elektromagnetische Untersuchungen durchgeführt. Die Messungen fanden allerdings auf Profilen statt, die sich hauptsächlich auf dessen östlichen Teil konzentrierten (JöDICKE et al., 1983; VOLBERS et al., 1990). Die Interpretationen beschränkten sich im wesentlichen auf die Resultate eindimensionaler Inversionsrechnungen, zweidimensionale Modelle wurden nur in Einzelfällen berechnet. Darüberhinaus konnten kaum signifikante Aussagen über die Struktur des oberen Mantels getroffen werden.

Im Rahmen des von der Deutschen Forschungsgemeinschaft geförderten Projektes „Elektromagnetische Abtastung des oberen Mantels unter der Eifel" wurde mit Hilfe eines großräumigen Stationsnetzes nun erstmals ein Datensatz bereitgestellt, der eine dreidimensionale Interpretation ermöglicht. Insgesamt 30 Stationen bedeckten eine Fläche von mehr als $14.000 \mathrm{~km}^{2}$ und gestatteten die Entwicklung eines Modells der dreidimensionalen Leitfähigkeitsverteilung unter dem Rheinischen Schiefergebirge. Das Stationsnetz wurde dabei so gestaltet, daß eine Auflösung des oberen Erdmantels möglich war.

Einige Ergebnisse wurden bereits in Arbeiten, die im Rahmen dieses Meßprojektes entstanden, vorgestellt (HÖNIG, 1998; LEIBECKER \& KURAS, 1998; SOYER, 1998).

In der vorliegenden Arbeit werden zunächst die Grundlagen der elektromagnetischen Tiefenforschung erläutert. Die Möglichkeiten und Grenzen der Erkundung von inhomogenen Leitfähigkeitsstrukturen im Untergrund werden diskutiert. Dabei werden zur Analyse der magnetischen Daten einige neue Ansätze vorgestellt und erläutert.

Nach einer kurzen Einführung in die geologische Entwicklung des Meßgebiets wird anschließend das Design des Meßarrays präsentiert. Es folgt eine Beschreibung der verwendeten Methoden zur Aufzeichnung und Verarbeitung der elektromagnetischen Zeitreihen. Die wichtigsten Resultate der Analyse der gewonnenen Daten werden ausführlich erörtert. Abschließend werden dreidimensionale Modelle der Leitfähigkeitsverteilung unter dem Rheinischen Schiefergebirge vorgestellt und diskutiert. Sie bieten eine Erklärung für die wesentlichen Merkmale in den beobachteten magnetischen und elektrischen Daten. 


\section{Grundlagen der elektromagnetischen Tiefenforschung}

\subsection{Das anregende Feld}

Das erdmagnetische Hauptfeld wird von Schwankungen überlagert, die ihren Ursprung nicht im Erdinneren haben. Sie werden als geomagnetische Variationen bezeichnet.

Die Ursache dieser Fluktuationen sind elektrische Ströme in der Ionosphäre und Magnetosphäre. Diese sind nicht beständig und entstehen durch die solare Strahlung sowie die Wechselwirkung des solaren Windes mit der Magnetosphäre. In Tabelle 1.1 sind einige geomagnetische Variationen aufgelistet. Sie umfassen einen Periodenbereich von mehreren Zehnerpotenzen, wobei die größten beobachteten Amplituden in mittleren Breiten von wenigen $100 \mathrm{nT}$ im Vergleich zum Hauptfeld (am Äquator $\approx 31.000 \mathrm{nT}$ ) sehr klein sind.

Diese magnetischen Wechselfelder induzieren in der leitfähigen Erde sekundäre Stromsysteme. Durch Messung der elektrischen und magnetischen Feldgrößen an der Erdoberfläche kann mit den Methoden der elektromagnetischen Tiefenforschung die Leitfähigkeitsverteilung im Erdinneren bestimmt werden.

Es kann davon ausgegangen werden, daß die Anregung nur durch tangential-elektrische Quellenfelder (TE-Mode ${ }^{1}$ ) erfolgt (SCHMUCKER \& WEIDELT, 1975), so daß $E_{z}=0$ ist. Vertikale Ströme werden etwa durch Blitze in der unteren Atmosphäre erzeugt. Die dabei angeregten Schwingungen sind allerdings so kurzperiodisch, daß sie im Rahmen dieser Arbeit keine Rolle spielen.

\footnotetext{
${ }^{1}$ Jedes divergenzfreie Vektorfeld $\nabla \cdot \mathbf{F}=0$ läßt sich durch die Rotation eines Vektorpotentials darstellen: $\mathbf{F}=\nabla \times \mathbf{P}$.

Für die Diffusion in $z$-Richtung kann $\mathbf{P}$ in zwei linear unabhängige Teillösungen $\mathbf{P}_{1}=\psi \mathbf{e}_{z}$ und $\mathbf{P}_{2}=$ $\nabla \times \phi \mathbf{e}_{z}$ mit den Skalarpotentialen $\psi$ und $\phi$ aufgespalten werden. Dann wird $\mathbf{F}=\nabla \times \nabla \times\left(\phi \mathbf{e}_{z}\right)+$ $\nabla \times\left(\psi \mathbf{e}_{z}\right)=\mathbf{F}_{p}+\mathbf{F}_{t}$. Dabei bezeichnet $\mathbf{F}_{p}$ ein poloidales Feld und $\mathbf{F}_{t}$ ein toroidales Feld (ohne $z-$ Komonente). Die TE-Mode eines Feldes setzt sich dann zusammen aus dem toroidalen elektrischen Feld $\mathbf{E}_{t}$ und der zugehörigen poloidalen magnetischen Flußdichte $\mathbf{B}_{t}$. Die TM-Mode wird analog gebildet $\left(B_{z}=0\right)$.
} 


\begin{tabular}{|c|c|c|c|}
\hline Quellregion & Beispiel & Periode $^{2}$ & Amplitude $^{3}$ \\
\hline \multirow[t]{2}{*}{ Ionosphäre (E-Schicht) } & $\begin{array}{l}\text { Solare tägliche Variationen } \\
\text { (S bzw. Sq an magnetisch } \\
\text { ruhigen Tagen) }\end{array}$ & $1 \mathrm{~d}$ & $30-60 \mathrm{nT}$ \\
\hline & $\begin{array}{l}\text { Solar flare Effekte (sfe): } \\
\text { Kurzzeitige Verstärkung der } \\
\text { Sq-Stromsysteme }\end{array}$ & $10-20 \mathrm{~min}$ & $10 \mathrm{nT}$ \\
\hline \multirow[t]{2}{*}{$\begin{array}{l}\text { Ionosphäre, Magneto- } \\
\text { sphäre, Polarlichtzone }\end{array}$} & $\begin{array}{l}\text { Polare magnetische Stürme } \\
\text { (DP) }\end{array}$ & $10-120 \mathrm{~min}$ & $100 \mathrm{nT}$ \\
\hline & Polare Teilstürme (bay) & $30-120 \mathrm{~min}$ & $20-100 \mathrm{nT}$ \\
\hline \multirow[t]{3}{*}{ Magnetosphäre } & $\begin{array}{l}\text { Sudden storm commence- } \\
\text { ment (ssc): Starke Wechsel- } \\
\text { wirkung von solarem Wind } \\
\text { mit der Magnetopause }\end{array}$ & $2-5 \min$ & $10-100 \mathrm{nT}$ \\
\hline & $\begin{array}{l}\text { Very low frequency emissi- } \\
\text { ons (VLF) }\end{array}$ & $10^{-5}-10^{-3} \mathrm{~s}$ & $<0.1 \mathrm{nT}$ \\
\hline & $\begin{array}{l}\text { Ultra low frequency emissi- } \\
\text { ons (ULF) }\end{array}$ & $1-600 \mathrm{~s}$ & $0.1-10 \mathrm{nT}$ \\
\hline Atmosphäre, Gewitter & $\begin{array}{l}\text { Extra low frequency emissi- } \\
\text { ons (ELF) }\end{array}$ & $10^{-3}-10^{-1} \mathrm{~s}$ & $<0.1 \mathrm{nT}$ \\
\hline
\end{tabular}

Tabelle 1.1: Geomagnetische Variationen (vgl. SchMUCKER, 1984). Die Schwankungen mit Perioden $<600$ s werden auch als Pulsationen bezeichnet.

\subsection{Physikalische Grundlagen}

Die wichtigsten Beobachtungsgrößen in der elektromagnetischen Tiefenforschung sind die magnetische Flußdichte $\mathbf{B}$, in $\mathrm{Vsm}^{-2}=1 \mathrm{~T}^{4}$ und das elektrische Feld $\mathbf{E}$, in $\mathrm{Vm}^{-1}$. Sie werden üblicherweise an einem bestimmten Ort $(x, y, z)$ als Zeitreihen aufgezeichnet. Dabei sollen folgende Konventionen hinsichtlich der Koordinaten gelten: Die $x$-Richtung entspricht magnetisch Nord, die $z$-Richtung zeigt positiv nach unten. Alle Drehungen werden von „Nord nach Ost“ durchgeführt, das heißt, in Richtung der $z$-Achse in mathematisch positiver Rotationsrichtung (Abbildung 1.1).

\footnotetext{
${ }^{2}$ Grundperiode bei regelmäßigen Variationen, Periodenbereich bei unregelmäßigen Variationen.

${ }^{3}$ Amplitude in mittleren Breiten.

${ }^{4}$ Geomagnetische Variationen werden üblicherweise in Nanotesla gemessen: $1 \mathrm{nT}=10^{-9} \mathrm{~T}$. Eine ältere, zum Teil noch verwendete Einheit ist das Gauss: $1 \mathrm{G}=10^{5} \gamma(\mathrm{Gamma})=10^{-4} \mathrm{~T}$, also $1 \gamma=1 \mathrm{nT}$.
} 


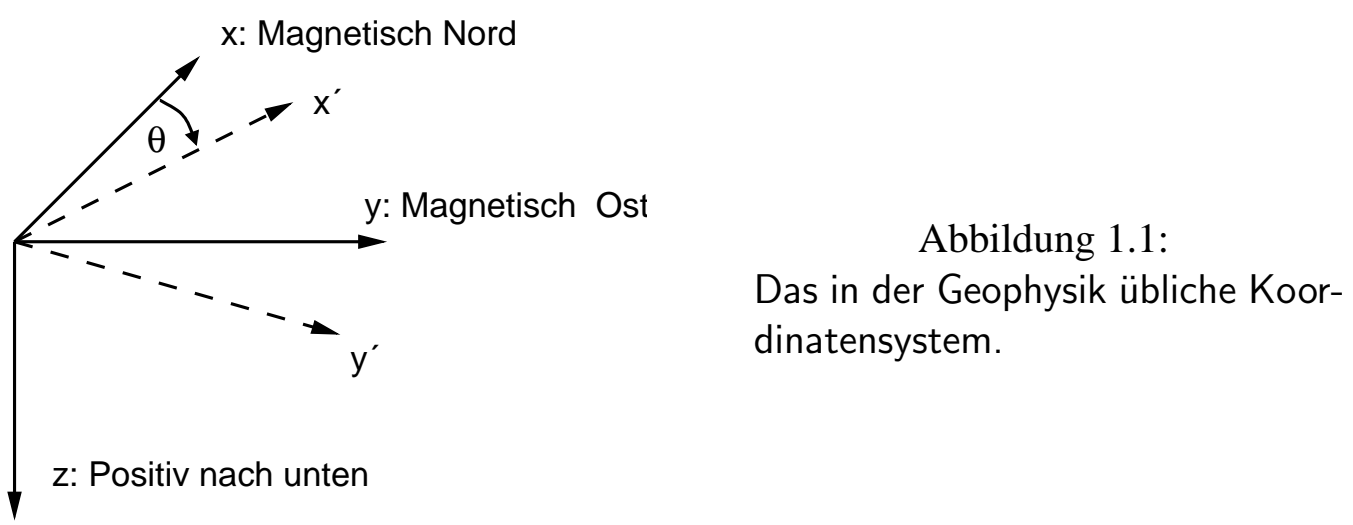

Alle gemessenen Feldgrößen stellen sich als nichtperiodische Vorgänge im Orts-ZeitBereich dar. Durch eine Fouriertransformation in den Frequenzbereich können einige Betrachtungen wesentlich vereinfacht und übersichtlicher dargestellt werden: $\mathbf{F}(\mathbf{r}, t) \rightarrow$ $\tilde{\mathbf{F}}(\mathbf{r}, \omega)$ mit $\mathbf{F}=\mathbf{E}, \mathbf{B}$. Dabei geht die Zeitabhängigkeit in eine Abhängigkeit von der Kreisfrequenz $\omega=2 \pi f$ über. Das Fourierintegral ist:

$$
\begin{aligned}
\mathbf{F}(\mathbf{r}, t) & =\frac{1}{\sqrt{2 \pi}} \int_{-\infty}^{\infty} \tilde{\mathbf{F}}(\mathbf{r}, \omega) e^{-i \omega t} d \omega \quad \text { mit } \\
\tilde{\mathbf{F}}(\mathbf{r}, \omega) & =\frac{1}{\sqrt{2 \pi}} \int_{-\infty}^{\infty} \mathbf{F}(\mathbf{r}, t) e^{i \omega t} d t
\end{aligned}
$$

Die Funktion im Zeitbereich $\mathbf{F}(\mathbf{r}, t)$ wird so als eine Überlagerung komplexer harmonischer Schwingungen $e^{-i \omega t}$ mit den Koeffizienten oder Gewichten $\tilde{\mathbf{F}}(\mathbf{r}, \omega)$ dargestellt. Man bezeichnet $\tilde{\mathbf{F}}(\mathbf{r}, \omega)$ daher auch als spektrale Amplitudendichte oder Spektraldichte. Die zeitliche Ableitung geht in eine Multiplikation mit dem Faktor $(-i \omega)$ über. In der elektromagnetischen Tiefenforschung ist die Darstellung des komplexen Spektrums $\tilde{\mathbf{F}}(\mathbf{r}, \omega)$ anstelle der Zeitfunktion $\mathbf{F}(\mathbf{r}, t)$ üblich. Falls nicht ausdrücklich anders erwähnt, finden alle weiteren Betrachtungen im Frequenzbereich statt. Daher wird im folgenden die Tilde weggelassen.

Untersuchungsgegenstand ist hier die feste Erde, so daß zunächst die Annahmen eines linearen und isotropen Mediums gerechtfertigt sind. Die magnetische Flußdichte B ist dann über die magnetische Permeabilität $\mu_{r}$ und die magnetische Feldkonstante $\mu_{0}$ mit der magnetischen Feldstärke $\mathbf{H}$, in $\mathrm{Am}^{-1}$, verknüpft ${ }^{5}$ :

$$
\mathbf{B}=\mu_{0} \mu_{r} \mathbf{H} .
$$

Entsprechend ergibt sich das elektrische Feld $\mathbf{E}$ mit der Dielektrizitätskonstanten $\varepsilon_{0}$ und der relativen Dielektrizität $\varepsilon_{r}$ aus der dielektrischen Verschiebung $\mathbf{D}$, in $\mathrm{As} \mathrm{m}^{-2}$ :

$$
\mathbf{E}=\left(\varepsilon_{0} \varepsilon_{r}\right)^{-1} \mathbf{D} .
$$

\footnotetext{
${ }^{5}$ In der Geophysik ist die Verwendung von B üblich. Soweit Verwechslungen ausgeschlossen sind, wird im folgenden $\mathbf{B}$ als magnetisches Feld bezeichnet.
} 
Die Konstanten $\mu_{0}$ und $\varepsilon_{0}$ hängen vom verwendeten Maßsystem ab. Im (hier ausschließlich verwendeten) SI-System ${ }^{6}$ ist $^{7}$ :

$$
\begin{aligned}
& \mu_{0}=4 \pi \cdot 10^{-7} \quad \mathrm{VsA}^{-1} \mathrm{~m}^{-1} \quad \text { und } \\
& \varepsilon_{0}=(4 \pi)^{-1} c^{-2} \cdot 10^{7}=8.8543 \cdot 10^{-12} \quad \mathrm{AsV}^{-1} \mathrm{~m}^{-1}
\end{aligned}
$$

Damit lauten die Maxwellschen Gleichungen:

$$
\begin{aligned}
\nabla \times \mathbf{E}-i \omega \mathbf{B} & =0 & & \text { (Faraday) } \\
\nabla \cdot \mathbf{B} & =0 & & \\
\nabla \times \mathbf{B}+i \omega \varepsilon_{0} \varepsilon_{r} \mu_{0} \mu_{r} \mathbf{E} & =\mu_{0} \mu_{r} \mathbf{j} & & \text { (Ampère) } \\
\nabla \cdot \mathbf{E} & =q / \varepsilon_{0} \varepsilon_{r} & & \text { (Coulomb) }
\end{aligned}
$$

$q$ und $\mathbf{j}$ bezeichnen die freie Raumladungs- bzw. Stromdichte. Ein wichtiger Materialparameter in der elektromagnetischen Tiefenforschung ist der

spezifische elektrische Widerstand $\rho$, in $\mathrm{VmA}^{-1}=\Omega \mathrm{m}$.

Sein Kehrwert wird als elektrische Leitfähigkeit $\sigma=\frac{1}{\rho}$ bezeichnet. In einem quellenfreien Medium gilt

$$
\mathbf{j}=\sigma \cdot \mathbf{E}
$$

Aus dieser Formulierung des Ohmschen Gesetzes in lokalen Größen geht hervor, daß die elektrische Leitfähigkeit in einem Ohmschen Leiter eine Materialkonstante ist, die nicht von dessen Geometrie und insbesondere nicht von der örtlichen elektrischen Feldstärke abhängt. Es ist zu beachten, daß die elektrische Leitfähigkeit im allgemeinen nicht nur orts- sondern auch richtungsabhängig ist: $\sigma \rightarrow \underline{\sigma}$. Diese Anisotropie der elektrischen Leitfähigkeit wird später noch diskutiert.

\subsection{Herleitung und Skalierung der Helmholtzgleichung}

Aus den Maxwellschen Gleichungen lassen sich in einfacher Weise Differentialgleichungen für das elektrische und magnetische Feld herleiten. Rotationsbildung von (1.1) ergibt mit (1.3) und (1.5)

$$
\nabla \times(\nabla \times \mathbf{E})-i \omega \mu_{0} \mu_{r} \sigma \mathbf{E}+\omega^{2} \varepsilon_{0} \varepsilon_{r} \mu_{0} \mu_{r} \mathbf{E}=0 .
$$

Um das Gewicht der einzelnen Terme zu bestimmen, wird die Gleichung skaliert. Das heißt, sämtliche Koeffizienten werden in dimensionslose Größen überführt. Sei $L$ eine

\footnotetext{
${ }^{6} \mathrm{Im}$ älteren Gaußschen Maßsystem ist beispielsweise $\varepsilon_{0}=\mu_{0}=1$.

${ }^{7}$ Mit $1 / \mu_{0} \varepsilon_{0}=c^{2}$, wobei $c=2.998 \cdot 10^{8} \mathrm{~ms}^{-1}$ die Lichtgeschwindigkeit im Vakuum bezeichnet.
} 
charakteristische Länge des Induktionsproblems. Dann erhält man mit $l^{\prime}=l / L$ eine dimensionslose Variable, sofern $l$ die Dimension einer Länge hat.

Damit ist $(\nabla \times)=1 / L\left(\nabla^{\prime} \times\right)$, und aus (1.6) ergibt sich:

$$
\nabla^{\prime} \times\left(\nabla^{\prime} \times \mathbf{E}\right)-\underbrace{i \omega \sigma L^{2} \mu_{0} \mu_{r}}_{\zeta_{1}} \cdot \mathbf{E}+\underbrace{\omega^{2} L^{2} \varepsilon_{0} \varepsilon_{r} \mu_{0} \mu_{r}}_{\zeta_{2}} \cdot \mathbf{E}=0 .
$$

Zur Abschätzung werden folgende Annahmen gemacht:

- $L=100 \mathrm{~km}$ (Ausdehnung des Meßgebiets)

- $\omega=1 \mathrm{~Hz}$ (kürzeste noch auswertbare Periode)

- $\sigma=10^{-4} \mathrm{Sm}^{-1}$ (kleinste Leitfähigkeit bei z.B. kristallinem Gestein)

- Für die meisten Materialien der Erde sind die Annahmen $\varepsilon_{r} \approx 1$ und $\mu_{r} \approx 1$ erlaubt. Einzig der Wert der relativen Dielektrizität für Wasser weicht mit $\varepsilon_{r} \approx 80$ von dieser Näherung ab. Die nachfolgenden Abschätzungen werden davon jedoch nicht beeinflußt. Zur Vereinfachung wird im folgenden $\varepsilon=\varepsilon_{0} \varepsilon_{r}$ und $\mu=\mu_{0} \mu_{r}$ geschrieben.

Mit diesen Zahlenwerten erhält man $\zeta_{1} \approx 1.3$ und $\zeta_{2} \approx 1.1 \cdot 10^{-7}$, so daß $\zeta_{1} / \zeta_{2} \approx 1.2 \cdot 10^{7}$. Im Rahmen der hier durchgeführten Untersuchungen kann also der zweite Term in (1.6) vernachlässigt werden. Er entspricht dem Maxwellschen Verschiebungsstrom $(-i \omega \varepsilon \mu \mathbf{E})$ im Ampèreschen Gesetz ${ }^{8}$. Zusammen mit (1.5) erhält man aus (1.3)

$$
\nabla \times \mathbf{B}=\mu \sigma \mathbf{E},
$$

woraus durch Divergenzbildung folgt:

$$
\nabla \cdot \mathbf{j}=0 .
$$

Mit (1.5) wird daraus

$$
\nabla \cdot(\sigma \mathbf{E})=\sigma \nabla \cdot \mathbf{E}+(\nabla \sigma) \cdot \mathbf{E}=0 .
$$

Bei einer Betrachtung im Zeitbereich folgt aus $\partial \mathbf{D} / \partial t=0$ unmittelbar $\partial q / \partial t=0$. Dies entspricht der Stationaritätsbedingung der Magnetostatik.

Man bezeichnet diese Näherung deshalb auch als

\section{quasi-stationäre Näherung.}

Physikalisch bedeutet dies, daß sich $q$ während der Zeit $\Delta t=L / c$, die die elektromagnetischen Wellen benötigen, um das Meßgebiet zu durchlaufen, vernachlässigbar wenig ändert. Aus (1.6) wird dann:

$$
\nabla \times(\nabla \times \mathbf{E})-\mu i \omega \sigma \mathbf{E}=0 .
$$

Analog erhält man die entsprechende Differentialgleichung für das magnetische Feld:

$$
\nabla \times((\nabla \times \mathbf{B}) / \sigma)-\mu i \omega \mathbf{B}=0 .
$$

\footnotetext{
${ }^{8}$ In der ursprünglichen Formulierung des Ampèreschen Gesetzes war der Verschiebungsstrom nicht enthalten.
} 


\subsection{Verhalten der Feldgrößen an Grenzflächen}

Die Gleichungen (1.9) und (1.10) beschreiben das Verhalten der Feldgrößen in der Erde, sofern die Leitfähigkeit homogen oder zumindest eine stetige Funktion des Ortes ist. Im allgemeinen wird es jedoch mehrere aneinander angrenzende Regionen mit unterschiedlichen Leitfähigkeiten geben. Zumindest muß jedes Modell der Erde den näherungsweise nicht leitfähigen Luftraum über dem leitfähigen Untergrund einbeziehen. Daher muß das Verhalten der Felder an Grenzflächen berücksichtigt werden. Die Randbedingungen können aus den Maxwellschen Gleichungen hergeleitet werden. Dazu wird, wie in Abbildung 1.2 zu erkennen, an der Grenzfläche zwischen zwei Medien aus zwei Flächenelementen $f$ ein Volumenelement $\Delta V$ gebildet. Der Abstand zwischen beiden Flächenelementen wird dabei (beliebig) klein gewählt. Durch Anwendung des Gaußschen Satzes ${ }^{9}$ auf das Coulombsche Gesetz erhält man:

$$
\oint_{S(\Delta V)} d \mathbf{F} \cdot \mathbf{E}=f \mathbf{n} \cdot\left(\mathbf{E}_{2}-\mathbf{E}_{1}\right)=\int_{\Delta V} d V q / \varepsilon=Q / \varepsilon .
$$

Mit der Oberflächenladungsdichte $\tilde{q}=Q / f$ folgt daraus:

$$
\mathbf{n} \cdot\left(\mathbf{E}_{2}-\mathbf{E}_{1}\right)=\tilde{q} / \varepsilon \text {. }
$$

Eine entsprechende Anwendung des Gaußschen Satzes auf $\nabla \cdot \mathbf{B}=0$ ergibt:

$$
\mathbf{n} \cdot\left(\mathbf{B}_{2}-\mathbf{B}_{1}\right)=0 .
$$
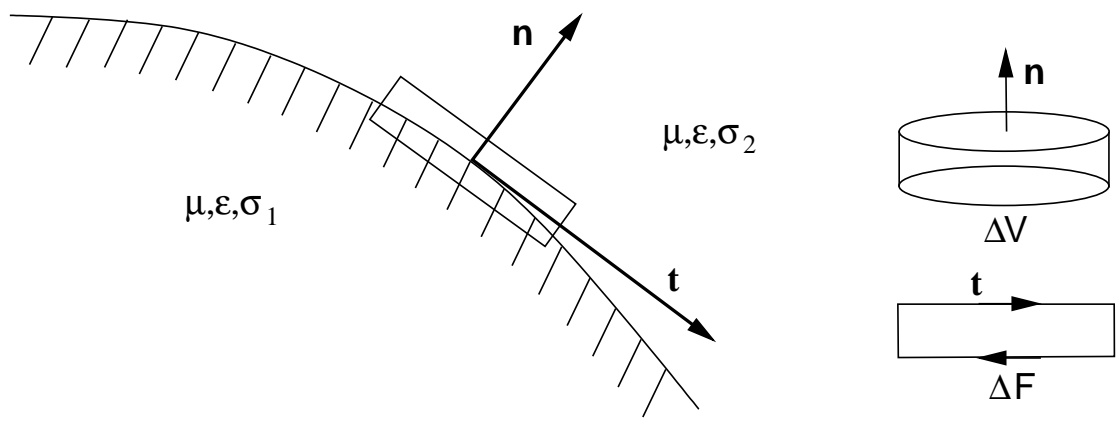

Abbildung 1.2: Schematische Darstellung einer Grenzfläche zwischen zwei Medien mit unterschiedlichen Leitfähigkeiten $\sigma_{1}$ und $\sigma_{2}$.

In ähnlicher Weise wird ein Flächenelement $\Delta F$ mit den Seitenlängen $l$ und beliebig kleinem Abstand gebildet. Die Anwendung des Stokesschen Satzes ${ }^{10}$ auf das Faradaysche

$$
\begin{gathered}
\int_{V} d V(\nabla \cdot \mathbf{A})=\oint_{S(V)} d \mathbf{F} \cdot \mathbf{A} \\
{ }_{F}^{10} d \mathbf{F} \cdot(\nabla \times \mathbf{A})=\oint_{C(F)} d \mathbf{r} \cdot \mathbf{A}
\end{gathered}
$$


und Ampèresche Gesetz führt zu:

$$
\begin{aligned}
& \mathbf{n} \times\left(\mathbf{B}_{2}-\mathbf{B}_{1}\right)=0 \quad \text { und } \\
& \mathbf{n} \times\left(\mathbf{E}_{2}-\mathbf{E}_{1}\right)=0 .
\end{aligned}
$$

Dabei wird vorausgesetzt, daß die Permeabilität und die relative Dielektrizitätskonstante in beiden Medien gleich sind. Darüberhinaus soll kein Oberflächenstrom vorhanden sein. Eine besondere Stetigkeitsbedingung, die allerdings nur bei Vernachlässigung der Verschiebungsströme, also bei Anwendung der quasi-stationären Näherung, Gültigkeit hat, ergibt sich aus (1.7). Die Normalkomponente der Stromdichte ist stetig, woraus

$$
\mathbf{n} \cdot\left(\sigma_{2} \mathbf{E}_{2}-\sigma_{1} \mathbf{E}_{1}\right)=0
$$

folgt. An der Grenzfläche springt die Normalkomponente des elektrischen Feldes gerade im reziproken Verhältnis der Leitfähigkeiten $E_{\perp}^{1} / E_{\perp}^{2}=\sigma_{2} / \sigma_{1}$.

\subsection{Induktion im homogenen Halbraum}

Die Induktionsgleichung soll zunächst für den Spezialfall eines homogenen isotropen Mediums gelöst werden. Dies hat seine Berechtigung darin, daß man sich die Erde aus einzelnen Bereichen zusammengesetzt denken kann, die zwar unterschiedliche Leitfähigkeiten besitzen, für sich genommen jedoch eine homogene Struktur aufweisen. Innerhalb eines solchen Blocks gilt:

$$
\nabla \sigma=0 .
$$

Aus (1.8) folgt dann unmittelbar

$$
\nabla \cdot \mathbf{E}=0
$$

In einem homogenen Medium ist also nicht nur $\mathbf{B}$, sondern auch $\mathbf{E}$ divergenzfrei. Das ist gleichbedeutend damit, daß keine freien Raumladungen existieren: $q=0$. Eine Akkumulation von Ladungen kann nur an Grenzflächen zwischen Regionen unterschiedlicher Leitfähigkeiten bzw. auf den Oberflächen homogener Teilbereiche auftreten. Die Induktionsgleichungen (1.9) und (1.10) vereinfachen sich damit zu der homogenen Helmholtzgleichung, die für das elektrische und magnetische Feld die gleiche funktionale Form aufweist:

$$
\nabla^{2} \mathbf{F}=-\mu \sigma i \omega \mathbf{F}
$$

mit $\mathbf{F}=\mathbf{E}, \mathbf{B}$. Sie beschreibt die Ausbreitung elektromagnetischer Wellen in einem leitfähigen homogenen Medium in Form eines Diffusionsvorganges. Mit der Annahme von senkrecht einfallenden ebenen Wellen wird (1.11) durch $\mathbf{F}(z)=\mathbf{F}_{0} e^{-i \kappa z}$ mit $\kappa^{2}=-\mu \sigma i \omega$ gelöst. Die Ausbreitungsrichtung der Welle ist die $z$-Richtung (positiv nach unten). 
Als Maßstabsgröße wird noch die Skintiefe $p$ definiert. Sie hat die Dimension einer Länge und gibt im Fall des homogenen Halbraums die Tiefe an, in der die Amplitude des Feldes auf $1 / e$ der Amplitude an der Erdoberfläche abgeklungen ist ${ }^{11}$ :

$$
p=\sqrt{\frac{2}{\omega \mu \sigma}} .
$$

Die Skintiefe gibt einen ersten Anhaltspunkt für die erreichbare Erkundungstiefe mit Hilfe von elektromagnetischen Methoden. In Abbildung 1.3 sind für einige typische Perioden die Skintiefen in Abhängigkeit des spezifischen Widerstandes aufgetragen. Man erkennt beispielsweise, daß mit Perioden in der Größenordnung einer Tageslänge (etwa Sq-Variationen) Erkundungstiefen bis in den Erdmantel erreicht werden. Sehr kurzperiodische Anregungen können zur Abtastung der obersten Erdschichten von wenigen Metern Tiefe benutzt werden.

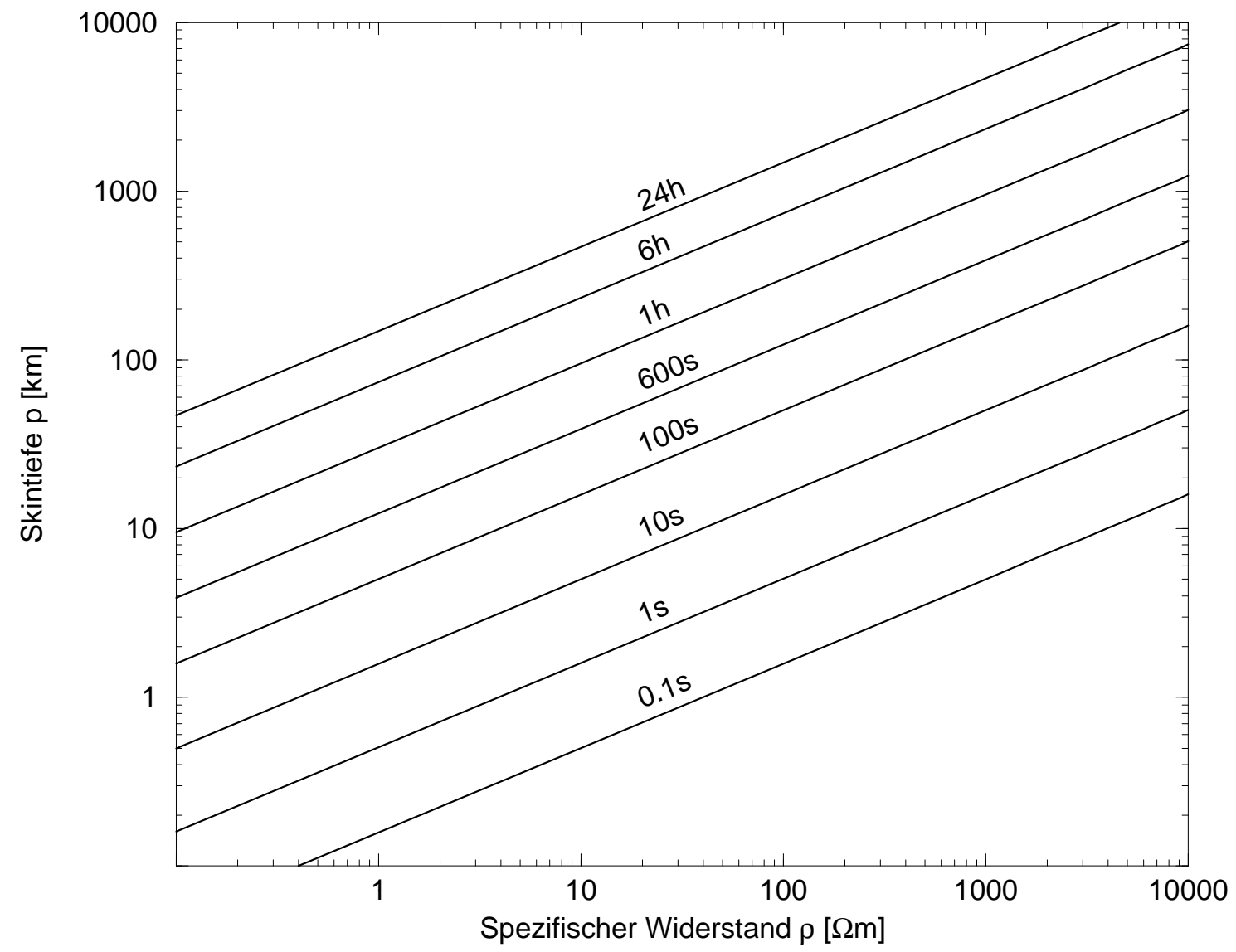

Abbildung 1.3: Skintiefen für verschiedene Frequenzen bei homogenem Untergrund.

${ }^{11}$ In der Tiefe $z=p$ ist $\mathbf{F}(p)=\mathbf{F}_{0} e^{-(1+i)}$. 
Zur weiteren Diskussion des Induktionsproblems wird eine Transformation der Horizontalkomponenten vom Orts-Frequenz-Bereich in den Wellenzahl-Frequenz-Bereich durchgeführt:

$$
\begin{aligned}
\hat{\mathbf{F}}\left(z, k_{x}, k_{y}, \omega\right) & =\frac{1}{2 \pi} \int_{-\infty}^{\infty} \mathbf{F}(z, x, y, \omega) e^{-i\left(k_{x} x+k_{y} y\right)} d x d y \quad \text { und } \\
\mathbf{F}(z, x, y, \omega) & =\frac{1}{2 \pi} \int_{-\infty}^{\infty} \hat{\mathbf{F}}\left(z, k_{x}, k_{y}, \omega\right) e^{i\left(k_{x} x+k_{y} y\right)} d k_{x} d k_{y} .
\end{aligned}
$$

Dabei bezeichnet $\hat{\mathbf{F}}\left(z, k_{x}, k_{y}, \omega\right)$ die komplexe Fourieramplitude als Funktion des ebenen Wellenzahlvektors $\mathbf{k}=k_{x} \mathbf{e}_{x}+k_{y} \mathbf{e}_{y}$. Über die reelle Wellenzahl $k=|\mathbf{k}|$ läßt sich eine räumliche Wellenlänge $\lambda=2 \pi / k$ bestimmen. Sie kann als eine horizontale ,Wellungslänge“ des Feldes in Ebenen $z=$ konst. verstanden werden.

Die Diffusionsgleichung (1.11) wird im Wellenzahlbereich zu

$$
\frac{d^{2}}{d z^{2}} \hat{\mathbf{F}}=\left(i \omega \mu \sigma+k^{2}\right) \hat{\mathbf{F}}=K^{2} \hat{\mathbf{F}}
$$

Dabei wird $K:=\sqrt{i \omega \mu \sigma+k^{2}}$ auch als komplexe vertikale Wellenzahl bezeichnet. Als Lösungsansatz für (1.13) kann

$$
\hat{\mathbf{F}}\left(z, k_{x}, k_{y}, \omega\right)=\hat{\mathbf{F}}_{0} e^{-K z}
$$

dienen. Dabei ist bereits berücksichtigt, daß die Felder beim Eindringen in die Erde kontinuierlich abklingen sollen: $\hat{\mathbf{F}}=0$ für $z \rightarrow \infty$. Die Messungen finden an der Erdoberfläche $z=0$ statt, dort wird $\hat{\mathbf{F}}=\hat{\mathbf{F}}_{0}$. Als erweiterte Maßstabslänge der Diffusion wird die komplexe Eindringtiefe

$$
C:=\frac{1}{K}
$$

definiert. Sie ist mit der Skintiefe (1.12) in folgender Weise verknüpft:

$$
C(k, \omega)=1 / \sqrt{i \omega \mu \sigma+k^{2}}=1 / \sqrt{2 i / p^{2}+k^{2}} .
$$

Man kann nun zwei Grenzfälle unterscheiden:

- Für $p^{2} k^{2} \gg 1$ oder $p \gg \lambda$ ist $C=1 / k$. Die komplexe Eindringtiefe ist dann unabhängig von den Eigenschaften des Untergrundes und wird nur durch die Wellenzahl $k$ des Quellenfeldes bestimmt. Das Feld diffundiert ohne Induktion durch das Medium wie durch einen Nichtleiter. In diesem Grenzfall können durch elektromagnetische Sondierungen keine Informationen über den Untergrund gewonnen werden. 
- Für $p^{2} k^{2} \ll 1$ oder $p \ll \lambda$ ist $C=\frac{1-i}{2} p$. In diesem Fall findet eine von der Wellenzahl $k$, also der räumlichen Struktur des Quellenfeldes, unabhängige Induktion statt. Formal kann in diesem Wellenzahlbereich $k=0$ gesetzt werden, weil für $k \rightarrow 0$ bzw. $\lambda \rightarrow \infty$ die komplexe Eindringtiefe $C(k, \omega)$ gegen einen Genzwert $C^{0}(\omega)$ strebt (Abbildung 1.4). Anschaulich bedeutet dies, daß im Bereich des Meßgebiets räumliche Variationen des Quellenfeldes vernachlässigt werden können. Man spricht dann von einer

\section{quasi-homogenen Anregung.}

Nur in diesem induktiven Grenzfall kann ohne zusätzliche Kenntnisse der Quellenfeldstruktur die elektrische Leitfähigkeit des Untergrundes bestimmt werden.

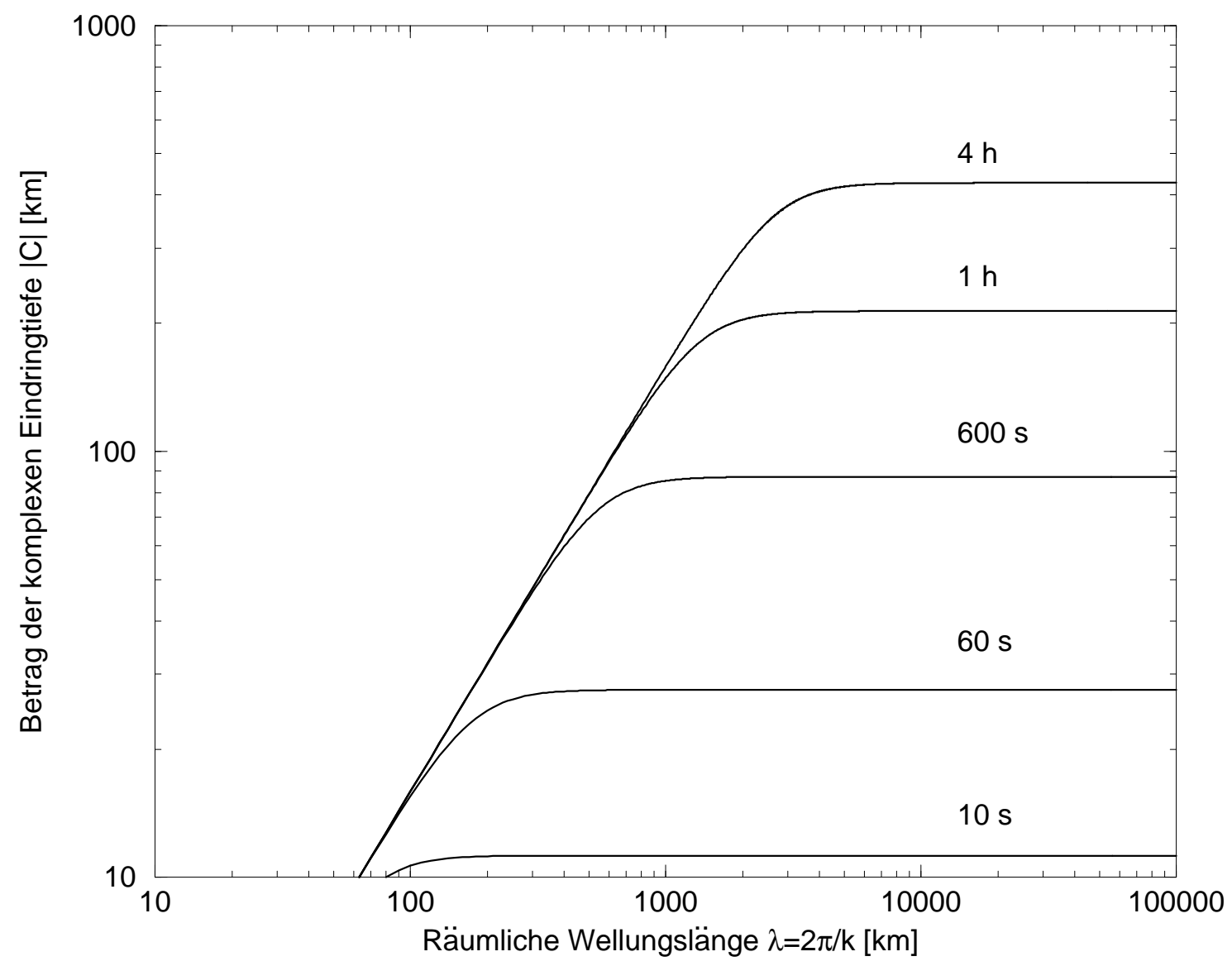

Abbildung 1.4: Abhängigkeit der komplexen Eindringtiefe von der horizontalen Wellungslänge für einen Halbraum von $100 \Omega \mathrm{m}$.

Um nachzuweisen, daß obige Annahme gerechtfertigt ist, muß man die Größenordnung der Wellungslänge abschätzen. In erster Näherung kann dazu die räumliche Halbwertsbreite der Feldintensität im Meßgebiet verwendet werden. In der Nähe der Quellregion beträgt sie für $B_{z}$ etwa $600 \mathrm{~km}$ (PETER, 1994). In Abbildung 1.4 erkennt man, daß in diesem 
Fall bereits ab Perioden von etwa 600 s die Qellenfeldstrukur maßgeblich die Induktion beeinflußt. Je weiter man sich von den Quellen entfernt, desto stärker klingt die räumliche Inhomogenität des Quellenfeldes ab. Die entsprechend anzusetzende Wellungslänge wird also größer. Die hier vorgestellten Messungen fanden in mittleren Breiten, also in großen Entfernungen von den Quellen statt. Man kann dann von einem sehr großräumig variierenden Feld ausgehen, so daß repräsentative Wellungslängen in der Größenordnung von etwa $6000 \mathrm{~km}$ liegen. Die Näherung für quasi-homogene Felder darf somit verwendet werden, und die Extrapolation $C(k, \omega) \rightarrow C^{0}(\omega)$ ist erlaubt. Aus diesem Grund wird der Index im folgenden weggelassen und nur $C(\omega)$ geschrieben.

Aus dem Ampèreschen Gesetz und dem Lösungsansatz (1.14) ergeben sich dann die folgenden linearen Beziehungen zwischen den jeweils orthogonalen Oberflächenfeldern:

$$
\hat{E}_{x}=i \omega C \hat{B}_{y} \quad \text { und } \quad \hat{E}_{y}=-i \omega C \hat{B}_{x} .
$$

Die Grundgleichungen der elektromagnetischen Tiefenforschung wurden ursprünglich im Wellenzahl-Frequenz-Bereich konzipiert.

Im folgenden soll davon ausgegangen werden, daß sich das Wellenzahlspektrum des Oberflächenfeldes auf den Bereich beschränkt, in dem von einer quasi-homogenen Anregung ausgegangen werden darf. Sofern die Leitfähigkeit konstant ist oder nur von der Tiefe abhängt $\sigma=\sigma(z)$, stellen sich alle Meßgrößen als frequenzabhängige, allein von der Leitfähigkeit bestimmte Größen dar. Die weiteren Betrachtungen können somit im OrtsFrequenz-Bereich stattfinden, der der unmittelbaren Anschauung etwas zugänglicher ist.

\subsection{Magnetotellurik}

Bei der Methode der Magnetotellurik werden das erdelektrische (=tellurische) Feld und die natürlichen Magnetfeldvariationen an der Erdoberfläche gemessen. Die daraus abgeleitete Übertragungsfunktion zwischen diesen Feldgrößen wird als komplexe Impedanz ${ }^{12}$ bezeichnet:

$$
Z(\omega)=\frac{E_{x}}{B_{y}}=-\frac{E_{y}}{B_{x}}=i \omega C=(1+i) \sqrt{\frac{\omega}{2 \mu \sigma}} .
$$

Aus dieser Beziehung können zwei wichtige Darstellungsgrößen der Magnetotellurik abgeleitet werden:

- Die zeitliche Phasenverschiebung der einzelnen Feldkomponenten ergibt sich mit $\mathbf{E}=|E| e^{i \phi_{E}}$ und $\mathbf{B}=|B| e^{i \phi_{B}}$ als Phase der Impedanz:

$$
\phi=\phi_{E}-\phi_{B}=\arg \{Z\}=\arctan \left(\frac{\operatorname{Im}\{Z\}}{\operatorname{Re}\{Z\}}\right) .
$$

\footnotetext{
${ }^{12}$ In der elektromagnetischen Tiefenforschung ist die Definition mit der magnetischen Flußdichte $B$ üblich. Die Impedanz hat dann die Dimension einer Geschwindigkeit. Bei Verwendung des magnetischen Feldes $H$ erhält sie die Einheit $\Omega$.
} 
Im Falle des homogenen Halbraums sieht man aus (1.15) $\operatorname{Im}\{Z\}=\operatorname{Re}\{Z\}$. Daraus ergibt sich $\phi=\arctan 1=45^{\circ}$.

- Der Widerstand des homogenen Halbraums läßt sich unmittelbar aus (1.15) mit $\rho=1 / \sigma$ berechnen. Auch wenn der Untergrund nicht homogen ist, kann man dem Betrag der Impedanz formal den Widerstand eines bei dieser Frequenz äquivalenten Halbraums zuordnen und erhält den sogenannten scheinbaren spezifischen Widerstand (CAGNIARD, 1953):

$$
\rho_{a}=\frac{\mu}{\omega}|Z|^{2}=\omega \mu|C|^{2} .
$$

Die Phase der Impedanz wird dabei nicht berücksichtigt.

Für den Fall des eindimensionalen Halbraums sind die beiden Größen nicht voneinander unabhängig, sondern durch Dispersionsbeziehungen miteinander verknüpft (WEIDELT, 1972). Die Phase der Impedanz kann aus dem Frequenzgang des scheinbaren spezifischen Widerstandes bestimmt werden, umgekehrt kann $\rho_{a}$ aus $\phi$ jedoch nur bis auf eine additive Konstante $\rho_{0}$ berechnet werden.

\section{Geschichteter Halbraum}

Der betrachtete Halbraum bestehe aus N-1 Schichten mit den jeweiligen Widerständen $\rho_{1} \ldots \rho_{N-1}$, Schichtdicken $d_{1} \ldots d_{N-1}$ und oberen Schichtgrenzen $z_{1} \ldots z_{N-1}$. Die Erdoberfläche liegt bei $z_{1}=0$. Der nach unten abschließende homogene Halbraum habe den Widerstand $\rho_{N}$. Ein erweiterter Lösungsansatz der Diffusionsgleichung, der auch aufwärts diffundierende Wellen zuläßt, beschreibt den Diffusionsvorgang in der $n$-ten Schicht:

$$
E(z)=A_{n} e^{-K_{n} z}+B_{n} e^{K_{n} z} .
$$

Dabei bezeichnet $K_{n}=\sqrt{i \omega \mu \sigma_{n}}$ die vertikale Wellenzahl der $n$-ten Schicht. Unter Ausnutzung der Stetigkeitsbedingungen an den inneren Grenzflächen können die Einzellösungen verbunden werden, und man erhält schließlich einen Ausdruck, der die Übertragungsfunktion $C_{n}$ an der Obergrenze der $n$-ten Schicht in Abhängigkeit der Übertragungsfunktion der darunterliegenden $(n+1)$-ten Schicht angibt:

$$
C_{n}=\frac{1}{K_{n}} \cdot \frac{C_{n+1} K_{n}+\tanh \left(K_{n} d_{n}\right)}{1+C_{n+1} K_{n} \tanh \left(K_{n} d_{n}\right)} .
$$

Dies ist die Grundformel des Wait-Algorithmus (WAIT, 1953). Im unten abschließenden Halbraum dürfen nur abwärts diffundierende Felder vorkommen, dort ist $C_{N}=1 / K_{N}$. Ausgehend von diesem Startwert lassen sich auf rekursivem Weg die Übertragungfunktionen der darüberliegenden Schichten bestimmen, bis man letztendlich die gewünschte Übertragungsfunktion $C_{1}$ (entspricht hier dem $C$ in Kapitel 1.5) an der Erdoberfläche erhält.

Der einfachste Fall besteht aus einem Halbraum mit einer Deckschicht. Zwei Spezialfälle sind dabei denkbar, von denen ausgehend SCHMUCKER (1987) die $\rho^{*}\left(z^{*}\right)$-Transformation einführte: 
- $\tau$-Modell

Über dem schlechtleitenden $\left(\left|K_{2}\right| d_{1} \ll 1\right)$ Halbraum liege eine gut leitfähige dünne Deckschicht mit der integrierten Leitfähigkeit $\tau=d_{1} / \rho_{1}$. Der Widerstand des Halbraums ergibt sich zu:

$$
\rho^{*}=\frac{\rho_{a}}{2 \sin ^{2} \varphi}
$$

Die integrierte Leitfähigkeit ist: $\tau=(\cos \varphi-\sin \varphi) / \mu|Z|$.

- $h$-modell

Umgekehrt soll nun der Halbraum gegenüber der Deckschicht gut leitend sein $\left(p_{2} \leq p_{1}\right)$. Für den Widerstand des Halbraums ergibt sich dann:

$$
\rho^{*}=\rho_{a} \cdot 2 \cos ^{2} \varphi .
$$

Für die Dicke der Deckschicht erhält man $h=d_{1}=(\sin \varphi-\cos \varphi) \cdot|Z| / \omega$.

Jeder Meßwert zu einer bestimmten Frequenz läßt sich durch ein äquivalentes $h$ - oder $\tau$-Modell erklären. Die Modellzuordnung geschieht dabei durch die Phase der Impedanz. Um negative Leitfähigkeiten oder Schichtdicken zu vermeiden, ersieht man aus (1.18) und (1.19) unmittelbar, daß sich für $0 \leq \varphi \leq \pi / 4$ das $\tau$-Modell und für $\pi / 4 \leq \varphi \leq \pi / 2$ das $h$-Modell ergibt. Dabei wurde vorausgesetzt, daß die Phase nur im ersten Quadranten liegt. Für den Fall einer reinen Tiefenabhängigkeit der Leitfähigkeit ist diese Annahme erlaubt (WEIDELT, 1994). Falls $\varphi=\pi / 4$ ist, fehlt die Deckschicht, das heißt, der Halbraum ist homogen.

Es ist möglich, den scheinbaren Widerständen $\rho^{*}$ eine Tiefe

$$
z^{*}=\operatorname{Re}(C)
$$

zuzuordnen, die als Schwerpunkttiefe der induzierten Stromsysteme interpretiert werden kann (WEIDELT, 1972). Eine Auftragung von $\rho^{*}\left(z^{*}\right)$ ergibt bereits eine gute Näherung für die wahren Widerstandsverhältnisse in den jeweiligen Tiefen.

\subsection{Erdmagnetische Tiefensondierung}

Beim Verfahren der Erdmagnetischen Tiefensondierung werden im Unterschied zur Methode der Magnetotellurik nur die magnetischen Feldkomponenten zur Interpretation verwendet. Es ist die gleichzeitige Registrierung an mindestens zwei Stationen erforderlich. Der Methode liegt folgende Modellvorstellung zugrunde: Die Leitfähigkeitsverteilung des Untergrundes soll sich aus einer geschichteten Normalstruktur $\sigma^{n}$ und einem anomalen Anteil $\sigma^{a}$ zusammensetzen:

$$
\sigma=\sigma^{n}(z)+\sigma^{a}(x, y, z) .
$$


Die an der Erdoberfläche gemessenen Felder bestehen dann ebenfalls aus einem normalen und einem anomalen Anteil:

$$
\mathbf{E}=\mathbf{E}^{n}+\mathbf{E}^{a} \quad \text { und } \quad \mathbf{B}=\mathbf{B}^{n}+\mathbf{B}^{a} .
$$

Der normale Feldanteil entspricht dem Feld, welches ohne Anomalie $\left(\sigma^{a}=0\right)$ oder ausreichend weit von ihr entfernt gemessen würde. Man wählt nun eine Referenzstation und bezieht die Felder aller anderen Stationen auf diese so definierten Normalfelder. Mit der Annahme eines linearen Zusammenhangs zwischen den Feldkomponenten kann folgende Beziehung definiert werden (SCHMUCKER, 1970):

$$
\left(\begin{array}{c}
B_{x}^{a} \\
B_{y}^{a} \\
B_{z}^{a}
\end{array}\right)=\underline{\mathbf{W}}\left(\begin{array}{c}
B_{x}^{n} \\
B_{y}^{n}
\end{array}\right) .
$$

Dabei bezeichnet $\underline{\mathbf{W}}$ eine Matrix aus frequenzabhängigen Übertragungsfunktionen:

$$
\underline{\mathbf{W}}=\left(\begin{array}{ll}
h_{H} & h_{D} \\
d_{H} & d_{D} \\
z_{H} & z_{D}
\end{array}\right) .
$$

$\underline{\text { W }}$ wird auch als Perturbationsmatrix oder -tensor bezeichnet. Die einzelnen Elemente ${ }^{13}$ sind komplexwertig. In der Darstellung (1.20) ist bereits berücksichtigt, daß es über einem geschichteten Halbraum kein vertikales Magnetfeld gibt: $B_{z}^{n}=0$. Das gesamte beobachtete vertikale Magnetfeld ist also in diesem Sinne anomal. Eine Aufteilung der vertikalen Feldkomponente in einen normalen und einen anomalen Anteil ist deshalb überflüssig: $B_{z}^{a}=B_{z}$. Deswegen ist es aber auch sinnvoll, das gemessene vertikale Magnetfeld direkt auf das horizontale Feld derselben Station zu beziehen. Man definiert so lokale Übertragungsfunktionen

$$
B_{z}=z_{H}^{l} B_{x}+z_{D}^{l} B_{y}
$$

im Unterschied zu den oben dargestellten normalen Übertragungsfunktionen, die immer auf eine Referenzstation bezogen sind.

Geht man von einer homogenen Anregung aus, so enthalten die Elemente der Perturbationsmatrix nur Informationen über innere Strukturen des Untergrundes. Inhomogenitäten des äußeren Feldes würden sich direkt in den Normalanteilen abbilden und so Effekte erzeugen, die nicht unmittelbar erkennen lassen, daß sie äußeren Ursprungs sind.

Die Elemente des Perturbationstensors zeigen direkt den anomalen Stromfluß der untersuchten Station an und sind deshalb einer unmittelbaren Interpretation gut zugänglich. Folgende Modellstudie illustriert das räumliche Verhalten der magnetischen Übertragungsfunktionen über einer Leitfähigkeitsanomalie. Der Aufbau des Modells ist in Abbildung 1.5 dargestellt.

${ }^{13}$ Die Bezeichnungen der Tensorelemente leiten sich von den in der Geophysik ebenfalls gebräuchlichen Bezeichnungen $(H, D, Z)$ für $\left(B_{x}, B_{y}, B_{z}\right)$ her. 
Modell V
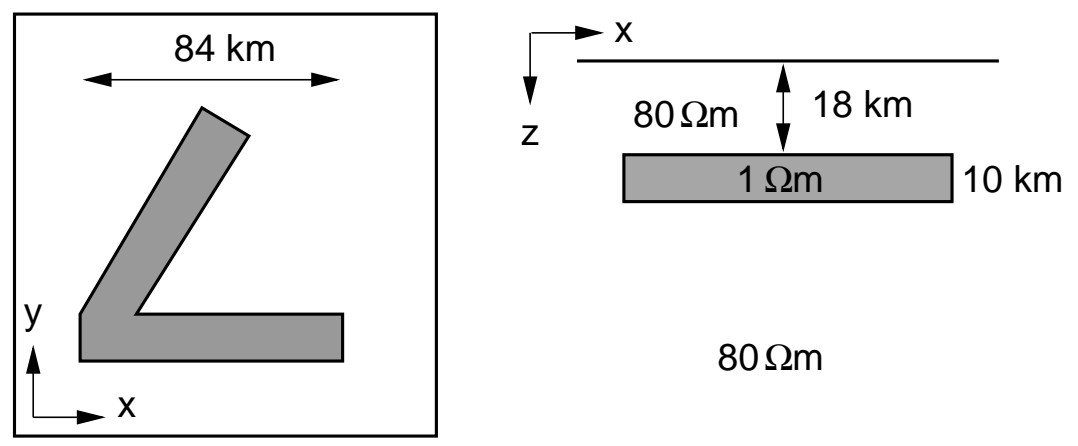

$80 \Omega m$

Abbildung 1.5: Aufbau des Modells V.

Es besteht aus einem V-förmigen Körper, der in einen homogenen Halbraum eingebettet ist. Der vollständige Perturbationstensor wurde an mehreren Stationen für eine bestimmte Periode berechnet.

In Abbildung 1.6 sind die Ergebnisse für die Realteile bei einer Periode von $85 \mathrm{~s}$ dargestellt. Die berechneten Übertragungsfunktionen besitzen im Frequenzgang bei dieser Periode ein Maximum. Das Modell wurde außerdem so dimensioniert, daß an den beiden Seiten des Körpers die Effekte einer zweidimensionalen Struktur aufgezeigt werden können. Damit ist gemeint, daß die Länge einer Seite deutlich größer als die Eindringtiefe des elektromagnetischen Feldes bei Perioden um $85 \mathrm{~s}$ ist . Bei einer Station, die etwa in der Mitte einer Seite liegt, kann davon ausgegangen werden, daß sie in diesem Sinne von einer zweidimensionalen Anomalie beeinflußt wird.

Man erkennt, daß die Realteile der Hauptdiagonalelemente ${ }^{14}$ direkt über den entsprechenden Seiten des Körpers ein Maximum durchlaufen und dann relativ schnell abklingen.

Die räumliche Ausdehnung des anomalen horizontalen Feldes ist im wesentlichen auf den Bereich des Induktionskörpers beschränkt. Dagegen zeigen die Realteile der Vertikalkomponenten $z_{H}$ und $z_{D}$ direkt über der Anomalie einen Nulldurchgang, und das Maximum bzw. Minimum wird außerhalb der eigentlichen anomalen Struktur erreicht. Eine Leitfähigkeitsanomalie kann also das vertikale Magnetfeld noch in beträchtlicher Entfernung beeinflussen. Der Abstand zwischen Minimum und Maximum bei einer Profilauftragung von $B_{z}$ kann Hinweise auf die Tiefenlage der Anomalie geben. Je oberflächenaher eine zweidimensionale Struktur liegt, desto geringer wird der Abstand.

Die Nebendiagonalelemente sind überall klein. Auffällig ist, daß sich im $\operatorname{Re}\left\{h_{D}\right\}$ an den Ecken der Struktur lokale Maxima bzw. Minima zeigen.

\footnotetext{
${ }^{14}$ Wenn im folgenden der Perturbationstensor $\underline{\mathbf{W}}$ erwähnt wird, sind im allgemeinen nur die horizontalen Übertragungsfunktionen gemeint. Nebendiagonalelemente sind also $h_{D}$ und $d_{H}$, die Hauptdiagonalelemente entsprechend $h_{H}$ und $d_{D}$.
} 

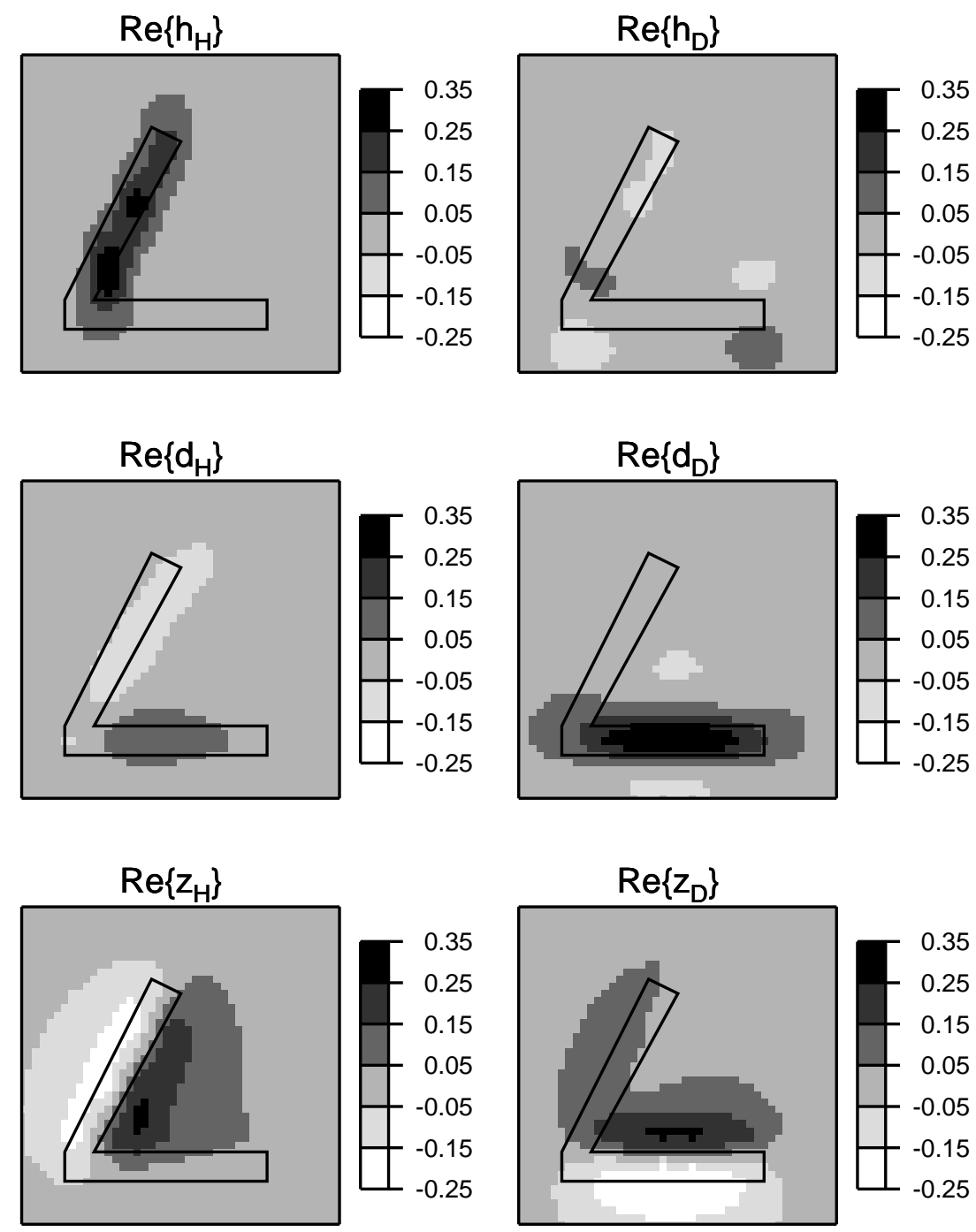

Abbildung 1.6: Die Realteile aller Elemente des Perturbationstensors inklusive der vertikalen Übertragungsfunktionen für Modell V (Abbildung 1.5) bei einer Periode von $85 \mathrm{~s}$.

Die Übertragungsfunktionen für das vertikale Magnetfeld werden oft in Form von sogenannten Induktionspfeilen $^{15}$ (IP) dargestellt. Dabei werden in der Regel die lokalen Übertragungsfunktionen verwendet:

$$
\mathbf{I P}=z_{H}^{l} \mathbf{e}_{x}+z_{D}^{l} \mathbf{e}_{y}
$$

Man bezeichnet den aus Realteilen gebildeten Pfeil auch als Realpfeil, den anderen entsprechend als Imaginärpfeil. Trägt man sie in einer Karte über lateralen Leitfähigkeits-

${ }^{15}$ Auch die Bezeichnung Induktionsvektor ist üblich. Sie ist allerdings irreführend, da beispielsweise eine Vektoraddition von Induktionspfeilen physikalisch nicht korrekt ist. Nur bei induktiv schwach gekoppelten Anomalien ist sie näherungsweise möglich (SIEMON, 1997). 
kontrasten auf, so zeigen die Pfeile vom guten Leiter weg ${ }^{16,17}$.

Ganz entsprechend werden Perturbationspfeile aus den horizontalen Übertragungsfunktionen des Magnetfeldes gebildet:

$$
\begin{aligned}
& \mathbf{p}=h_{H} \mathbf{e}_{x}+d_{H} \mathbf{e}_{y} \\
& \mathbf{q}=h_{D} \mathbf{e}_{x}+d_{D} \mathbf{e}_{y} .
\end{aligned}
$$

Rotiert man diese Pfeile um $90^{\circ}$ gegen den Uhrzeigersinn, so zeigen sie Stärke und Richtung des anomalen Stromflusses an, welcher dem nordwärts $(\mathbf{q})$ bzw. westwärts (p) fließenden normalen Stromfluß überlagert ist (SCHMUCKER, 1970).
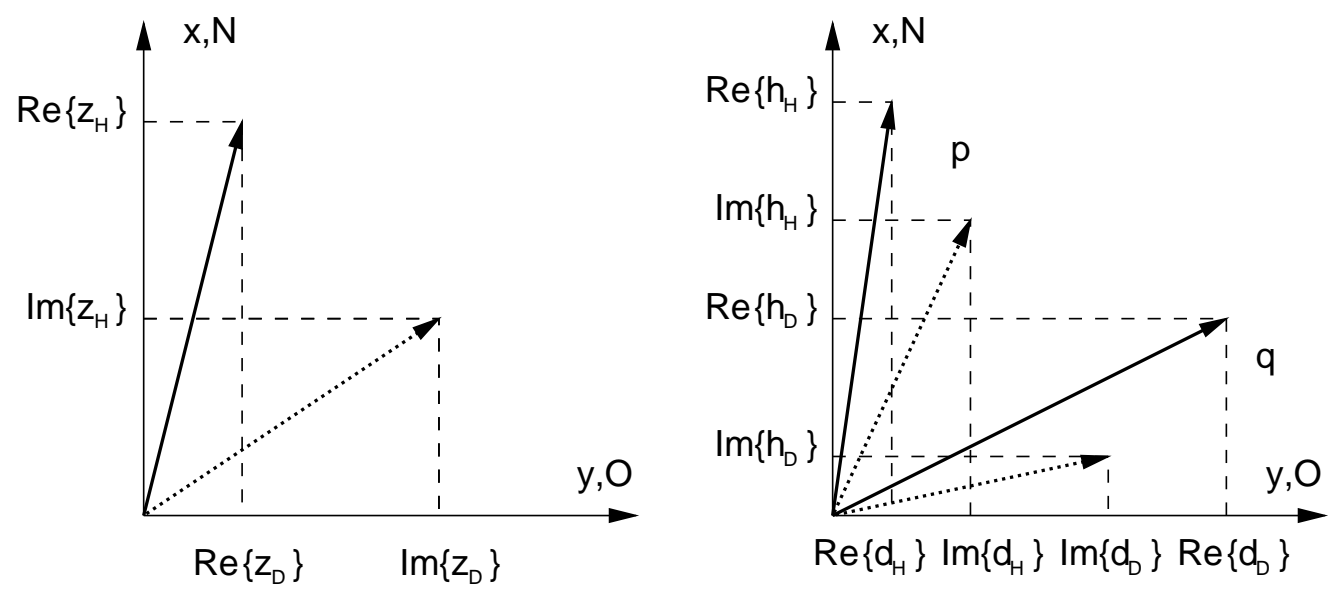

Abbildung 1.7: Die Darstellung magnetischer Übertragungsfunktionen in Form von Induktionspfeilen und Perturbationspfeilen.

\subsection{Verzerrung elektromagnetischer Felder durch lokale Störkörper}

Im folgenden soll das Problem der elektromagnetischen Streuung an einem Körper mit dem Volumen $V$ im homogenen Medium betrachtet werden. Zur Lösung der Helmholtzgleichung werden das elektrische Feld und die Leitfähigkeit in anomale und normale Anteile aufgespalten. Es ist also $\sigma^{a}(\mathbf{r})=\sigma(\mathbf{r})-\sigma^{n}$ und $\mathbf{E}^{a}(\mathbf{r})=\mathbf{E}(\mathbf{r})-\mathbf{E}^{n}(\mathbf{r})$. Die Differentialgleichung für das elektrische Feld lautet dann (WEAVER, 1994):

$$
\nabla \times(\nabla \times \mathbf{E}(\mathbf{r}))-\mu i \omega \sigma^{n} \mathbf{E}(\mathbf{r})=\mu i \omega \sigma^{a}(\mathbf{r}) \mathbf{E}(\mathbf{r})
$$

\footnotetext{
${ }^{16}$ Das gilt gleichermaßen, wenn die einzelnen Elemente rotiert und die Pfeile im neuen Koordinatensystem dargestellt werden.

${ }^{17}$ Konzept nach WIESE (1962). Bei einer Auftragung der Pfeile nach PARKINSON (1959) zeigen sie zum guten Leiter hin.
} 
Der Term $\mu i \omega \sigma^{a}(\mathbf{r}) \mathbf{E}(\mathbf{r})$ kann als an dem Störkörper gestreutes Stromfeld aufgefaßt werden.

Drückt man das anomale elektrische Feld mit Hilfe Greenscher Funktionen aus, ergibt sich

$$
\mathbf{E}^{a}(\mathbf{r})=i \omega \mu \int_{V} \underline{\mathbf{G}}\left(\mathbf{r}, \mathbf{r}^{\prime}\right) \sigma^{a}\left(\mathbf{r}^{\prime}\right) \mathbf{E}\left(\mathbf{r}^{\prime}\right) d \mathbf{r}^{\prime}
$$

Dabei bezeichnet $\mathbf{r}^{\prime}$ den Ortsvektor zu einem Quellpunkt, das heißt zu einem Punkt innerhalb der Anomalie. Der Vektor $\mathbf{r}^{\prime}$ kennzeichnet den Beobachtungspunkt. Die zugehörige dyadische Greensche Funktion muß dann

$$
\nabla \times \nabla \times \underline{\mathbf{G}}\left(\mathbf{r}, \mathbf{r}^{\prime}\right)-\mu i \omega \sigma^{n} \underline{\mathbf{G}}\left(\mathbf{r}, \mathbf{r}^{\prime}\right)=\underline{\mathbf{I}} \delta\left(\mathbf{r}, \mathbf{r}^{\prime}\right)
$$

erfüllen, wobei I die Einheitsmatrix darstellt. Mit dem Ansatz (Kong, 1975)

$$
\underline{\mathbf{G}}\left(\mathbf{r}, \mathbf{r}^{\prime}\right)=\left(\underline{\mathbf{I}}+\frac{1}{k^{2}} \nabla_{r} \nabla_{r}\right) g\left(\mathbf{r}, \mathbf{r}^{\prime}\right) \quad \text {, wobei } \quad k^{2}=\mu i \omega \sigma^{n} \quad \text { ist },
$$

vereinfacht sich (1.24) zu einer inhomogenen Helmholtzgleichung

$$
\left(\nabla^{2}+k^{2}\right) g\left(\mathbf{r}, \mathbf{r}^{\prime}\right)=-\delta\left(\mathbf{r}, \mathbf{r}^{\prime}\right)
$$

mit der skalaren Greenschen Funktion $g\left(\mathbf{r}, \mathbf{r}^{\prime}\right)$. In Kugelkoordinaten lautet die Lösung (Kong, 1975; ARFKEN, 1985):

$$
g\left(\mathbf{r}, \mathbf{r}^{\prime}\right)=\frac{e^{i k\left|\mathbf{r}-\mathbf{r}^{\prime}\right|}}{4 \pi\left|\mathbf{r}-\mathbf{r}^{\prime}\right|} .
$$

Damit ergibt sich für das elektrische Feld innerhalb und außerhalb des Störkörpers folgende Integralgleichung:

$$
\begin{array}{r}
\mathbf{E}(\mathbf{r})=\mathbf{E}^{n}(\mathbf{r})+i \omega \mu \int_{V} g\left(\mathbf{r}, \mathbf{r}^{\prime}\right) \sigma^{a}\left(\mathbf{r}^{\prime}\right) \mathbf{E}\left(\mathbf{r}^{\prime}\right) d \mathbf{r}^{\prime}+ \\
\frac{1}{\sigma^{n}} \nabla_{r} \nabla_{r} \cdot \int_{V} g\left(\mathbf{r}, \mathbf{r}^{\prime}\right) \sigma^{a}\left(\mathbf{r}^{\prime}\right) \mathbf{E}\left(\mathbf{r}^{\prime}\right) d \mathbf{r}^{\prime} .
\end{array}
$$

In dieser Formulierung wird das gestreute elektrische Feld durch die Summe von zwei Integraltermen dargestellt, die nichtlinear in $\sigma^{a}(\mathbf{r})$ sind. Der erste Term beschreibt den induktiven Effekt des Störkörpers, der zweite eine galvanische Streuung durch Ladungsanhäufung an Grenzflächen.

Zur Lösung des Problems kann eine Linearisierung mit Hilfe der Bornschen Näherung (BORN, 1933) vorgenommen werden. Dabei wird das elektrische Feld in den Integraltermen durch das normale elektrische Feld ersetzt: $\mathbf{E} \approx \mathbf{E}^{n}$. Dieser Ansatz ist allerdings nur anwendbar bei Problemstellungen, bei denen der Leitfähigkeitskontrast $\sigma^{a}$ nicht zu groß 
und solange der Streukörper relativ klein ist, bzw. die betrachteten Perioden lang genug sind (BORN \& WOLF, 1980).

Deshalb schlugen HABASHY et al. (1993) eine Erweiterung der Bornschen Näherung vor: Das elektrische Feld innerhalb der Inhomogenität wird dabei beschrieben durch

$$
\mathbf{E}\left(\mathbf{r}^{\prime}\right)=\Gamma\left(\mathbf{r}^{\prime}\right) \cdot \mathbf{E}^{n}\left(\mathbf{r}^{\prime}\right) \approx \Gamma\left(\mathbf{r}^{\prime}\right) \cdot \mathbf{E}^{n}(\mathbf{r})
$$

wobei

$$
\Gamma(\mathbf{r})=\left[\mathbf{I}-\left(i \omega \mu \mathbf{I}+\frac{1}{\sigma^{n}} \nabla_{r} \nabla_{r}\right) \int_{V} g\left(\mathbf{r}, \mathbf{r}^{\prime}\right) \sigma^{a}\left(\mathbf{r}^{\prime}\right) d \mathbf{r}^{\prime}\right]^{-1} .
$$

Innerhalb des Körpers kann das Feld räumlich variieren, das elektrische Normalfeld soll sich aber über der Inhomogenität nicht ändern und kann so am Ort des Störkörpers durch den Wert am Beobachtungspunkt ersetzt werden. Im Grenzfall sehr langer Perioden $\omega \rightarrow 0$ wird der Term reell, und das elektrische Feld im Störkörper ist dann in Phase mit dem elektrischen Normalfeld. Insgesamt erhält man

$$
\mathbf{E}(\mathbf{r})=\underline{\mathbf{C}}(\mathbf{r}) \cdot \mathbf{E}^{n}(\mathbf{r})
$$

mit

$$
\underline{\mathbf{C}}(\mathbf{r})=\mathbf{I}+\left(i \omega \mu \mathbf{I}+\frac{1}{\sigma^{n}} \nabla_{r} \nabla_{r}\right) \cdot \int_{V} g\left(\mathbf{r}, \mathbf{r}^{\prime}\right) \sigma^{a}\left(\mathbf{r}^{\prime}\right) \Gamma\left(\mathbf{r}^{\prime}\right) d \mathbf{r}^{\prime} .
$$

$\underline{\mathbf{C}}(\mathbf{r})$ wird als Verzerrungstensor des elektrischen Feldes bezeichnet. Hier werden nur die horizontalen Feldkomponenten betrachtet, so daß es sich um eine (2,2)-Matrix handelt. Sämtliche Elemente sind im allgemeinen Fall komplex. Nur falls der induktive Term in (1.25) sehr klein im Vergleich zum galvanischen Term ist, werden alle Elemente reell, und $\underline{\mathbf{C}}(\mathbf{r})$ beschreibt eine galvanische Verzerrung des elektrischen Feldes. Im Fall einer regionalen ein- oder zweidimensionalen Struktur können sich dann die Amplituden, nicht jedoch die Phasen der Übertragungsfunktionen ändern. Das nachfolgende Beispiel (Abbildung 1.8) verdeutlicht das. Hier wurde ein eindimensionales Modell mit und ohne lokalen Störkörper gerechnet und die Ergebnisse zusammen dargestellt.

Man erkennt, daß die Phasenkurven beider Polarisationen zunächst bis etwa $30 \mathrm{~s}$ aufspalten und sich dann der Kurve des eindimensionalen Modells nähern. Schließlich liegen alle Kurven deckungsgleich aufeinander und lassen sich nicht mehr unterscheiden. Bei sehr kurzen Perioden hat die Anomalie einen induktiven Einfluß und wirkt in diesem Sinne auch nicht als ,lokaler“ Störkörper.

Beim Frequenzgang des scheinbaren spezifischen Widerstandes spalten die beiden Polarisationen ebenfalls zu Beginn auf, laufen aber im Unterschied zu den Phasenkurven nicht wieder zusammen. Ab einer gewissen Periode (im Beispiel fast von Beginn an) ist die Aufspaltung frequenzunabhängig. Beide Kurven haben dann die gleiche Form und sind nur gegeneinander verschoben. Man spricht in diesem Fall auch von einem static shift. 

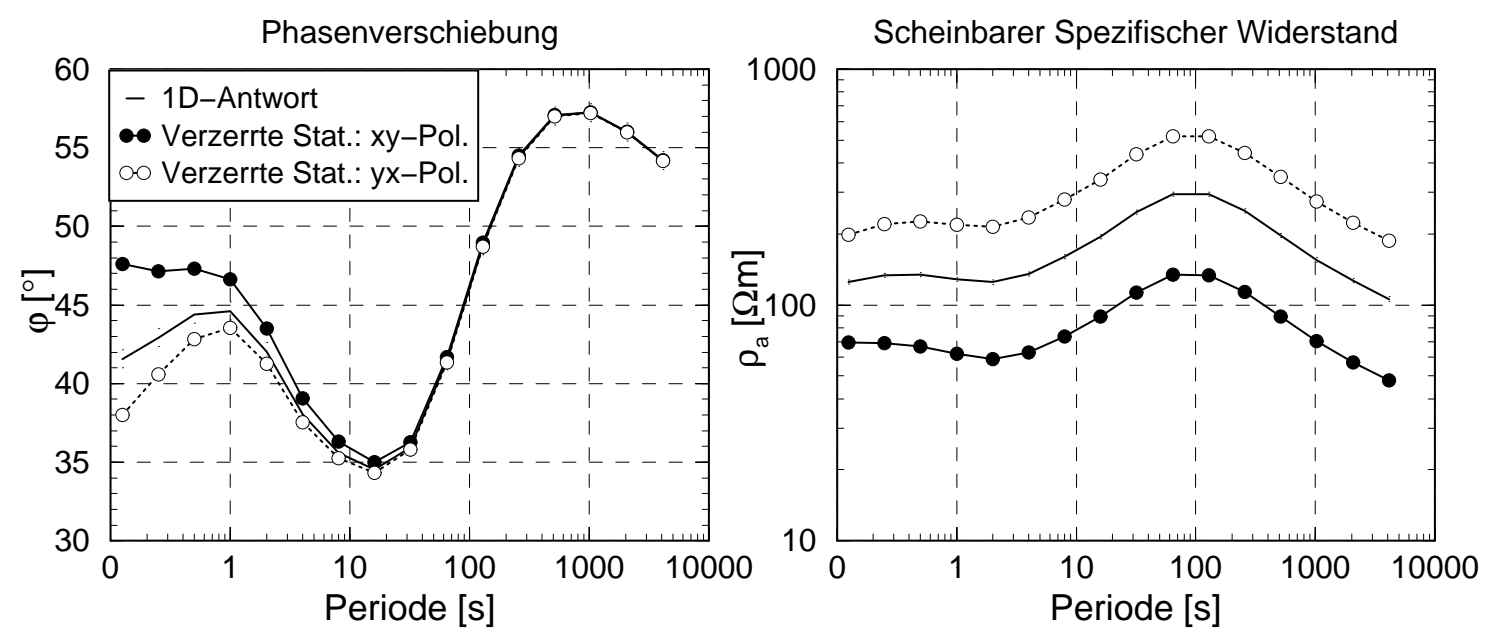

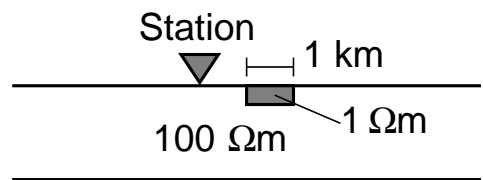

$300 \Omega m$
$8 \mathrm{~km}$

$68 \mathrm{~km}$
Abbildung 1.8:

Einfluß der galvanischen Verzerrung durch einen lokalen Störkörper auf den Frequenzgang der Phase und des scheinbaren spezifischen Widerstandes.

$50 \Omega m$

Der Verzerrungstensor des magnetischen Feldes kann in analoger Weise hergeleitet werden (Chave \& Smith, 1994). Die Anwendung des Ampèreschen Gesetzes auf (1.25) ergibt die Integralgleichung für das magnetische Feld:

$$
\mathbf{B}(\mathbf{r})=\mathbf{B}^{n}(\mathbf{r})+\mu \nabla \times \int_{V} g\left(\mathbf{r}, \mathbf{r}^{\prime}\right) \sigma^{a}\left(\mathbf{r}^{\prime}\right) \mathbf{E}\left(\mathbf{r}^{\prime}\right) d \mathbf{r}^{\prime} .
$$

Das anomale magnetische Feld entsteht durch räumliche Variationen des elektrischen Feldes $(\nabla \times \mathbf{E})$ und ist somit in Phase mit dem elektrischen Normalfeld (RITTER, 1996). Mit (1.26) erhält man

$$
\mathbf{B}(\mathbf{r})=\mathbf{B}^{n}(\mathbf{r})+\underline{\mathbf{D}}(\mathbf{r}) \cdot \mathbf{E}^{n}(\mathbf{r}),
$$

wobei

$$
\underline{\mathbf{D}}(\mathbf{r})=\mu \nabla \times \int_{V} g\left(\mathbf{r}, \mathbf{r}^{\prime}\right) \boldsymbol{\sigma}^{a}\left(\mathbf{r}^{\prime}\right) \Gamma\left(\mathbf{r}^{\prime}\right) d \mathbf{r}^{\prime} .
$$

Ebenso wie beim Verzerrungstensor des elektrischen Feldes $\underline{\mathbf{C}}(\mathbf{r})$ sind die Elemente von $\underline{\mathbf{D}}(\mathbf{r})$ im Grenzfall der galvanischen Verzerrung reell und hängen nur vom Beobachtungsort ab. 


\section{Strukturbestimmungen}

In Kapitel 1 wurden die Grundzüge der magnetotellurischen Methode anhand des eindimensionalen Falls behandelt. Die Impedanz (1.15) nimmt hier die einfache Gestalt einer Matrix $^{1}$ an, die nur aus zwei Elementen auf der Nebendiagonalen besteht. Beide haben die gleichen Beträge und unterscheiden sich nur durch das Vorzeichen:

$$
\underline{\mathbf{Z}}^{n}=\left(\begin{array}{cc}
0 & Z \\
-Z & 0
\end{array}\right)
$$

Die Hauptdiagonalelemente verschwinden unabhängig von der Wahl des Koordinatensystems, in dem gemessen wurde (Rotationssymmetrie). Sind die Nebendiagonalelemente sogar frequenzunabhängig, ist der Halbraum homogen.

Im allgemeinen wird die Leitfähigkeit nicht mehr nur tiefenabhängig sein, sondern darüber hinaus in den horizontalen Richtungen variieren. Dann können parallele Feldkomponenten miteinander korreliert sein, und der Impedanztensor ist auch auf der Hauptdiagonalen besetzt:

$$
\left(\begin{array}{c}
E_{x} \\
E_{y}
\end{array}\right)=\left(\begin{array}{ll}
Z_{x x} & Z_{x y} \\
Z_{y x} & Z_{y y}
\end{array}\right)\left(\begin{array}{c}
B_{x} \\
B_{y}
\end{array}\right)
$$

Entsprechend ist in diesem Fall der Perturbationstensor $\underline{\mathbf{W}}$ ebenfalls voll besetzt. Die zugehörigen Induktionsgleichungen sind dann in der Regel nur noch mit numerischen Methoden zu lösen.

Für das Verständnis der Zusammenhänge ist es sinnvoll, zunächst einige Spezialfälle zu betrachten. Es zeigt sich außerdem in der Praxis, daß ein Großteil der gemessenen Daten auf der Basis von relativ einfachen Modellvorstellungen interpretiert werden kann.

\footnotetext{
${ }^{1}$ In der Geophysik ist die Bezeichnung Impedanztensor üblich.
} 


\subsection{Modellvorstellungen in der elektromagnetischen Tiefenforschung}

\subsubsection{Die Nebendiagonalform des Impedanztensors}

Im Fall der reinen zweidimensionalen Leitfähigkeitsverteilung ändert sich die Streichrichtung in nur einer horizontalen Richtung. Hier soll sie in $y$-Richtung verlaufen, das heißt $\sigma=\sigma(x, z)$. Die Ableitungen der Felder in $y$-Richtung verschwinden dann $(\partial F / \partial y=0)$ und die Maxwellschen Gleichungen entkoppeln in zwei voneinander unabhängige Gruppen.

Die Gleichungen der ersten Gruppe beschreiben einen parallel zur Streichrichtung fließenden Strom, der von einem magnetischen Feld in der $(x, z)$-Ebene umgeben ist. Das elektrische Feld ist tangential zur Streichrichtung polarisiert und besitzt keine $z$-Komponente. Man bezeichnet diesen Fall als E-Polarisation.

Im umgekehrten Fall, der B-Polarisation, beschreiben die Gleichungen ein magnetisches Feld, das parallel zur Streichrichtung polarisiert ist, umgeben von einem elektrischen Feld in der $(x, z)$-Ebene. In Abbildung 2.1 ist dies dargestellt.

$$
\begin{gathered}
\text { E-Polarisation } \\
\frac{\partial}{\partial z} B_{x}-\frac{\partial}{\partial x} B_{z}=\mu \sigma E_{y} \\
\frac{\partial}{\partial z} E_{y}=-i \omega B_{x} \\
\frac{\partial}{\partial x} E_{y}=i \omega B_{z}
\end{gathered}
$$

\section{B-Polarisation}

$$
\begin{gathered}
\frac{\partial}{\partial x} B_{y}=-\mu \sigma E_{z} \\
\frac{\partial}{\partial z} B_{y}=-\mu \sigma E_{x} \\
\frac{\partial}{\partial z} E_{x}-\frac{\partial}{\partial x} E_{z}=i \omega B_{y}
\end{gathered}
$$
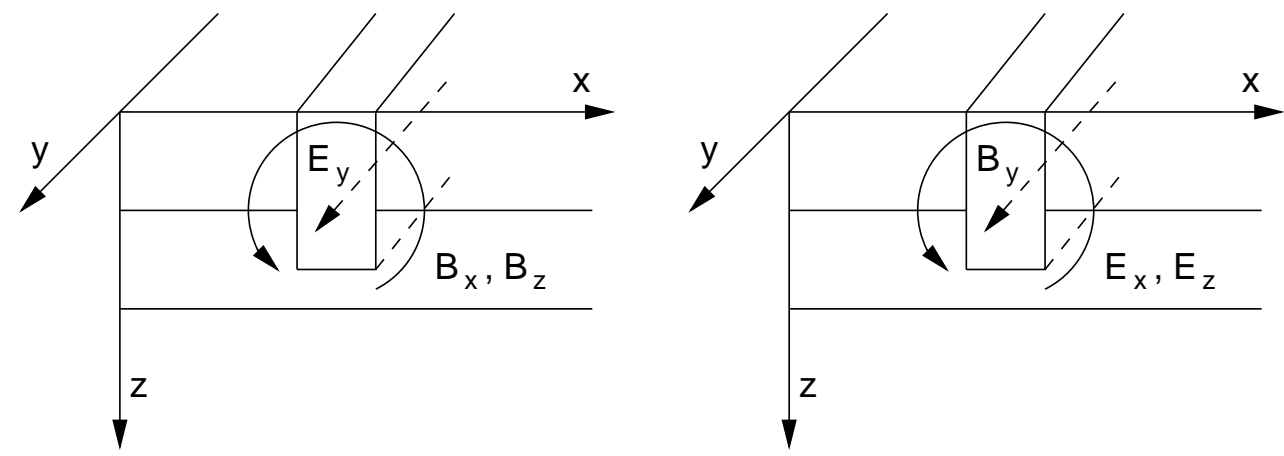

Abbildung 2.1: Eine schematische Darstellung der E- und B-Polarisation anhand einer isolierten 2D-Struktur in einem geschichtetem Halbraum.

Im Koordinatensystem der Streichrichtung ${ }^{2}$ sind die Hauptdiagonalelemente wie im eindimensionmalen Fall null, die Nebendiagonalelemente sind jedoch zudem untereinander

\footnotetext{
${ }^{2}$ Die Streichrichtung kennzeichnet im zweidimensionalen Fall die Richtung, in der sich die Leitfähigkeit nicht ändert.
} 
verschieden:

$$
\underline{\mathbf{Z}}=\left(\begin{array}{cc}
0 & Z_{x y} \\
Z_{y x} & 0
\end{array}\right)
$$

In diesem Beispiel gehört $Z_{x y}$ zur B-Polarisation und $Z_{y x}$ zur E-Polarisation. Es ist dabei zu beachten, daß im zweidimensionalen Fall durch Induktion in der Leitfähigkeitsanomalie ${ }^{3}$ auch sekundäre Felder in $z$-Richtung entstehen, die im eindimensionalen Fall (Gleichung 2.1) nicht auftreten.

In Abbildung 2.1 erkennt man bereits, daß ein anomales magnetisches Feld nur im Fall der E-Polarisation entsteht, da das Magnetfeld parallel zur Streichrichtung konstant ist, bzw. dem Normalfeld entspricht. Es ist $\mathbf{B}_{y}=\mathbf{B}_{y}^{n}$ und somit $\mathbf{B}_{y}^{a}=0$. Der Perturbationstensor besteht im richtigen Koordinatensystem nur aus einem von null verschiedenen Element auf der Hauptdiagonalen. Welches dies ist, hängt davon ab, ob die $x$ - oder die $y$-Richtung der Streichrichtung entspricht. Für dieses Beispiel ist:

$$
\underline{\mathbf{W}}=\left(\begin{array}{cc}
h_{H} & 0 \\
0 & 0
\end{array}\right) \text {. }
$$

\subsubsection{Anisotropie der Leitfähigkeit}

Legt man die Vorstellung einer zweidimensionalen Struktur zugrunde (s. Abbildung 2.1), so weist der Impedanztensor nur in der Nähe des Leitfähigkeitskontrastes die Form (2.3) auf. Nun wird der Meßpunkt in $x$-Richtung verschoben. Bei fortschreitender Entfernung von der 2D-Struktur nähert sich die jeweils bestimmte Impedanz dann immer mehr der eindimensionalen Form (2.1) an, weil der Einfluß der Anomalie immer geringer wird.

In der Praxis wird allerdings zum Teil der Fall beobachtet, daß ein Verschieben der Meßstation bzw. die Messung an mehreren Stationen in einem Array die grundsätzliche (zweidimensionale) Form der Impedanztensoren nicht ändert.

Diese Beobachtung führte zum Konzept der Anisotropie der elektrischen Leitfähigkeit. Die Leitfähigkeit kann in allen drei Raumrichtungen variieren. Mit den Methoden der elektromagnetischen Tiefenforschung können jedoch nur horizontale Komponenten erkannt werden, so daß im folgenden auschließlich auf die laterale Anisotropie eingegangen wird. Zur Erklärung der Beobachtungen werden zwei Modellvorstellungen herangezogen.

\section{Makroskopische Anisotropie}

Die an der Oberfläche beobachtete Anisotropie der Leitfähigkeit wird durch makroskopische Strukturen erzeugt, die in den Untergrund eingebettet sind und so großräumig eine Vorzugsrichtung des Stromflusses erzeugen. Das kann zum Beispiel eine Folge von Lamellen, Röhren oder ähnlichen zweidimensionalen Strukturen sein. Abbildung 2.2 zeigt eine schematische Darstellung des Lamellenmodells.

\footnotetext{
${ }^{3}$ In der Regel werden im Sprachgebrauch der elektromagnetischen Tiefenforschung alle Leitfähigkeitsverteilungen als anomal bezeichnet, die von einem eindimensionalen Halbraum abweichen.
} 


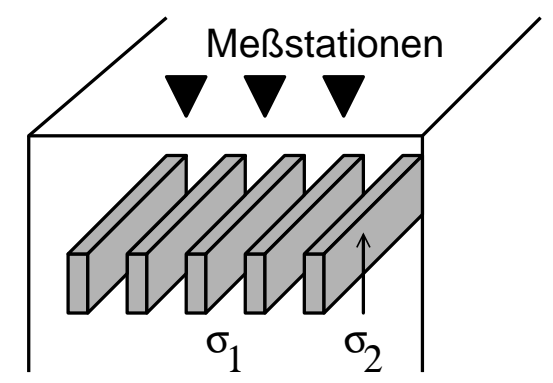

Abbildung 2.2: Schematische Darstellung der makroskopischen Anisotropie mit Hilfe des Lamellenmodells. $\sigma_{1}$ bezeichnet die Leitfähigkeit des umgebenden Mediums, $\sigma_{2}$ die Leitfähigkeit der Lamellen.

\section{Mikroskopische oder intrinsische Anisotropie}

Dieses Modell (Abbildung 2.3) beinhaltet die Vorstellung, daß die richtungsabhängige Leitfähigkeit dem untersuchten Material eingeprägt ist. Allgemein kann die richtungsabhängige Leitfähigkeit durch einen vollbesetzten (3,3)-Tensor beschrieben werden. Für geophysikalische Untersuchungen sind jedoch zwei Annahmen erlaubt, die die Form des Tensors vereinfachen. Falls $\underline{\sigma}$ positiv definit und symmetrisch ist, kann man den Leitfähigkeitstensor auf Diagonalgestalt transformieren (MAURER, 1993; DEKKER \& HASTIE, 1980):

$$
\underline{\sigma}^{\prime}=\left(\begin{array}{ccc}
\sigma_{x}^{\prime} & 0 & 0 \\
0 & \sigma_{y}^{\prime} & 0 \\
0 & 0 & \sigma_{z}^{\prime}
\end{array}\right) .
$$

Dabei kennzeichen $\sigma_{x}^{\prime}, \sigma_{y}^{\prime}$ und $\sigma_{z}^{\prime}$ die Leitfähigkeiten in den drei Hauptanisotropierichtungen $x^{\prime}, y^{\prime}$ und $z^{\prime}$. Diese bilden ein Orthogonalsystem (MAURER, 1993).

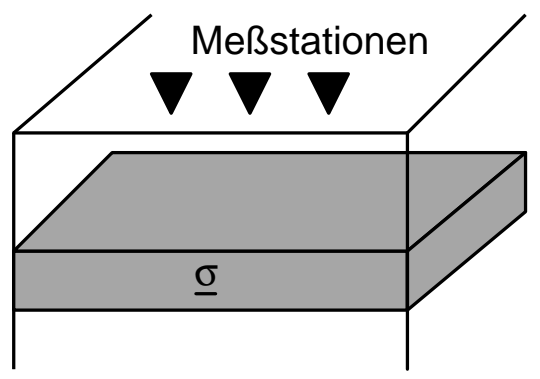

Abbildung 2.3: Schematische Darstellung der mikroskopischen Anisotropie. Die anisotrope Schicht besitzt eine Leitfähigkeit $\underline{\sigma}$ der Form (2.5).

Allein anhand von elektromagnetischen Daten ist es in der Praxis nicht möglich, zwischen beiden Modellen zu unterscheiden. Grundsätzlich können durch Messungen der Impedanz in zwei orthogonalen Richtungen nur die beiden horizontalen Hauptanisotropierichtungen bestimmt werden. 


\subsubsection{Regionale Induktion und galvanische Verzerrung}

Ein aus Felddaten bestimmter Impedanztensor wird in der Regel in keinem Koordinatensystem die Nebendiagonalform (2.1) oder (2.3) besitzen. Das kann mehrere mögliche Ursachen haben.

Zunächst ist es natürlich möglich, daß die untersuchte Leitfähigkeitsverteilung dreidimensional ist: $\sigma=\sigma(x, y, z)$.

Weiterhin sind die Meßdaten mehr oder weniger fehlerbehaftet, so daß auch bei einer 1D- oder 2D-Struktur in der Praxis alle Elemente des Impedanztensors von null verschieden sind. Liegen jedoch die Hauptdiagonalelemente in der Größe des Datenfehlers, kann dennoch davon ausgegangen werden, daß die Leitfähigkeitsverteilung nur ein- oder zweidimensional ist.

Als dritte Möglichkeit ist denkbar, daß die Meßdaten durch einen lokalen Störkörper im Sinne einer galvanischen Verzerrung beeinflußt werden ${ }^{4}$.

Die Verzerrung der elektromagnetischen Felder kann mit (1.27) und (1.29) im galvanischen Grenzfall durch zwei reelle Matrizen beschrieben werden. Hier werden nur die horizontalen Feldkomponenten betrachet, so daß es sich dabei um $(2,2)$-Matrizen handelt:

$$
\underline{\mathbf{C}}=\left(\begin{array}{ll}
C_{x x} & C_{x y} \\
C_{y x} & C_{y y}
\end{array}\right) \quad \text { und } \quad \underline{\mathbf{D}}=\left(\begin{array}{cc}
D_{x x} & D_{x y} \\
D_{y x} & D_{y y}
\end{array}\right)
$$

\section{Regionales 1D-Modell \& Verzerrung des E-Feldes}

Zunächst soll ausschließlich eine Verzerrung des elektrischen Feldes angenommen werden. Im Fall eines geschichteten Halbraums mit lokalem Störkörper setzt sich die gemessene Impedanz $\underline{\mathbf{Z}}$ aus der normalen, das heißt unverzerrten Impedanz $\underline{\mathbf{Z}}^{n}$ und der reellen Verzerrungsmatrix $\underline{\mathbf{C}}$ zusammen:

$$
\underline{\mathbf{Z}}=\underline{\mathbf{C}} \underline{\mathbf{Z}}^{n}
$$

Die explizite Form der Verzerrungsmatrix $\underline{\mathbf{C}}$ ist normalerweise von untergeordnetem Interesse. Sie wird jedoch in der Regel benötigt, um die unverzerrte Impedanz aus den gemessenen Übertragungsfunktionen zu extrahieren.

Es wurden mehrere Methoden zur Entzerrung des Impedanztensors entwickelt.

So kann versucht werden, mit Hilfe des Z:H-Verfahrens allein aus unverzerrten Magnetfeldern der Sq-Variationen eine normale Impedanz zu gewinnen. Anhand der so bestimmten Verzerrungsmatrix können dann die Impedanzen für kürzere Perioden entzerrt werden (LARSEN, 1977).

Existiert eine unverzerrte Station im Meßgebiet, so kann auch anhand dieser Daten eine Normalstruktur festgelegt werden, die für alle Stationen bei langen Perioden gültig ist und so zur Entzerrung benutzt werden kann.

\footnotetext{
${ }^{4}$ Unter einer lokalen Anomalie soll im folgenden eine im Vergleich zur Skintiefe sehr kleine Struktur verstanden werden. Davon abgesehen kann sie eine beliebige (3D) Geometrie aufweisen. Eine regionale Struktur soll in ihren horizontalen Dimensionen entsprechend großräumig sein.
} 
KEMMERLE (1977) versuchte durch oberflächennahe Abtastung des Untergrundes mit geoelektrischen Methoden zusätzliche Informationen über die lokale Anomalie zu gewinnen und so die Verzerrungsmatrix zu bestimmen.

\section{Regionales 2D-Modell \& Verzerrung des E-Feldes}

Der Ansatz (2.7) kann erweitert werden, indem anstelle eines geschichteten Halbraums eine zweidimensionale regionale Struktur mit einem Streichwinkel $\theta_{R}$ zugelassen wird (BAHR, 1988). Die regionalen Feldgrößen genügen dann dem Ausdruck $\mathbf{E}^{R}=\underline{\mathbf{Z}}^{R} \mathbf{B}^{R}$ und der gemessene Impedanztensor besitzt die Form:

$$
\underline{\mathbf{z}}=\underline{\mathbf{R}}_{\theta_{R}}\left(\begin{array}{ll}
C_{x x} & C_{x y} \\
C_{y x} & C_{y y}
\end{array}\right) \underbrace{\left(\begin{array}{cc}
0 & Z_{x y}^{R} \\
Z_{y x}^{R} & 0
\end{array}\right)}_{\underline{\mathbf{Z}}^{R}} \underline{\mathbf{R}}_{\theta_{R}}^{T} .
$$

$\underline{\mathbf{R}}$ bezeichnet die Rotationsmatrix ${ }^{5}$.

\section{Regionales 2D-Modell \& Verzerrung des E- und B-Feldes}

Berücksichtigt man nun noch eine Verzerrung des magnetischen Feldes $\mathbf{B}=\mathbf{B}^{R}+\underline{\mathbf{D}} \mathbf{E}^{R}$, erweitert sich der Ausdruck (2.8) zu der Form

$$
\underline{\mathbf{Z}}=\underline{\mathbf{R}}_{\theta_{R}} \underline{\mathbf{C}}^{R}\left(\underline{\mathbf{I}}+\underline{\mathbf{D}} \underline{\mathbf{Z}}^{R}\right)^{-1} \underline{\mathbf{R}}_{\theta_{R}}^{T}
$$

Der zusätzliche Faktor $\left(\underline{\mathbf{I}}+\underline{\mathbf{D}} \underline{\mathbf{Z}}^{R}\right)^{-1}$, der durch die Berücksichtigung der magnetischen Verzerrung entsteht, enthält die magnetische Verzerrungsmatrix $\underline{\mathbf{D}}$ gekoppelt mit der regionalen Impedanz $\underline{\mathbf{Z}}^{R}$ und ist dementsprechend komplex und frequenzabhängig. Im Gegensatz zum Fall der ausschließlichen Berücksichtigung der galvanischen Verzerrung des elektrischen Feldes kann hier auch die Phase der Impedanz beeinflußt werden.

Der Einfluß der magnetischen Verzerrung fällt jedoch mit $\sqrt{\omega}$ ab (GROOM \& BAILEY, 1991), so daß dessen Einfluß bisher in der Regel vernachlässigt wurde. Erst in jüngerer Vergangenheit wurde auch der Einfluß der magnetischen Verzerrung auf den Impedanztensor untersucht (CHAVE \& SMITH, 1994; SMITH, 1997).

\subsection{Rotationsinvariante Maßzahlen}

Um abzuschätzen, inwieweit sich die Daten zumindest in erster Näherung durch die genannten Modelle erklären lassen, ist es sinnvoll, Kennzahlen zu betrachten, die Abweichungen von bestimmten Modellen unmittelbar anzeigen. Insbesondere sollte es möglich

${ }^{5}$ Rotation einer Matrix $\underline{\mathbf{M}}$ um den Winkel $\theta$ :

$$
\underline{\mathbf{M}}^{\prime}=\underline{\mathbf{R}}_{\theta} \cdot \underline{\mathbf{M}} \cdot \underline{\mathbf{R}}_{\theta}^{T} \quad \text { mit } \quad \underline{\mathbf{R}}_{\theta}=\left(\begin{array}{cc}
\cos \theta & \sin \theta \\
-\sin \theta & \cos \theta
\end{array}\right) .
$$


sein, den Fall einer dreidimensionalen Leitfähigkeitsverteilung zu erkennen. Da zunächst eine eventuell vorhandene Streichrichtung nicht bekannt ist, bieten sich rotationsinvariante Kennzahlen an.

Ganz allgemein liegen die betrachteten Übertragungsfunktionen für die horizontalen Feldkomponenten als komplexe (2,2)-Matrizen vor:

$$
\underline{\mathbf{M}}=\left(\begin{array}{ll}
m_{11} & m_{12} \\
m_{21} & m_{22}
\end{array}\right)
$$

mit $\underline{\mathbf{M}}=\underline{\mathbf{Z}}, \underline{\mathbf{W}}$. Nach einer Rotation um den Winkel $\theta$ mit der Rotationsmatrix (2.9) lauten die Elemente im neuen Koordinatensystem (vgl. Abbildung 1.1) in expliziter Form:

$$
\begin{aligned}
& m_{11}^{\prime}=m_{11} \cos ^{2} \theta+\left(m_{12}+m_{21}\right) \sin \theta \cos \theta+m_{22} \sin ^{2} \theta \\
& m_{12}^{\prime}=m_{12} \cos ^{2} \theta-\left(m_{11}-m_{22}\right) \sin \theta \cos \theta-m_{21} \sin ^{2} \theta \\
& m_{21}^{\prime}=m_{21} \cos ^{2} \theta-\left(m_{11}-m_{22}\right) \sin \theta \cos \theta-m_{12} \sin ^{2} \theta \\
& m_{22}^{\prime}=m_{22} \cos ^{2} \theta-\left(m_{12}+m_{21}\right) \sin \theta \cos \theta+m_{11} \sin ^{2} \theta .
\end{aligned}
$$

Aus den einzelnen Elementen können durch Linearkombination neue Maßzahlen gebildet werden. Man kann zeigen, daß genau 7 linear unabhängige Linearkombinationen existieren, die gegenüber einer Rotation des Koordinatensystems invariant sind (SZARKA \& MENVIELLE, 1997) ${ }^{6}$.

Ein möglicher Satz relativ einfach aufgebauter rotationsinvarianter Maßzahlen lautet zum Beispiel:

- $r_{1}=\operatorname{Re}\left\{\left(m_{12}-m_{21}\right) / 2\right\}$

- $r_{2}=\operatorname{Im}\left\{\left(m_{12}-m_{21}\right) / 2\right\}$

- $r_{3}=\operatorname{Re}\left\{\left(m_{11}+m_{22}\right) / 2\right\}$

- $r_{4}=\operatorname{Im}\left\{\left(m_{11}+m_{22}\right) / 2\right\}$

- $r_{5}=\operatorname{det}(\operatorname{Re}\{\underline{\mathbf{M}}\})=\operatorname{Re}\left\{m_{11}\right\} \operatorname{Re}\left\{m_{22}\right\}-\operatorname{Re}\left\{m_{12}\right\} \operatorname{Re}\left\{m_{21}\right\}$

- $r_{6}=\operatorname{det}(\operatorname{Im}\{\underline{\mathbf{M}}\})=\operatorname{Im}\left\{m_{11}\right\} \operatorname{Im}\left\{m_{22}\right\}-\operatorname{Im}\left\{m_{12}\right\} \operatorname{Im}\left\{m_{21}\right\}$

- $r_{7}=\operatorname{Im}\left\{\operatorname{det}(\underline{\mathbf{M}})=\operatorname{Re}\left\{m_{11}\right\} \operatorname{Im}\left\{m_{22}\right\}-\operatorname{Re}\left\{m_{12}\right\} \operatorname{Im}\left\{m_{21}\right\}\right.$ $+\operatorname{Im}\left\{m_{11}\right\} \operatorname{Re}\left\{m_{22}\right\}-\operatorname{Im}\left\{m_{12}\right\} \operatorname{Re}\left\{m_{21}\right\}$.

Es gibt mehrere Sätze von sieben unabhängigen Rotationsinvarianten einer komplexen Matrix. Die Entscheidung, welchen man auswählt, hängt von der Art der Fragestellung ab. Nur wenige Maßzahlen des Impedanztensors besitzen beispielsweise eine unmittelbar einsichtige (geo-)physikalische Relevanz. In den folgenden Kapiteln werden einige vorgestellt.

\footnotetext{
${ }^{6}$ Eine reelle (2,2)-Matrix besitzt 3 rotationsinvariante Maßzahlen.
} 


\subsubsection{Anwendungen in der Magnetotellurik}

In der Magnetotellurik werden verschiedene rotationsinvariante Maßzahlen des Impedanztensors zur Interpretation verwendet.

YEE \& PAULSON (1987) führten eine Eigenwertzerlegung des Impedanztensors durch und benutzten reelle Eigenwerte $\sigma_{1,2}^{2}=1 / 2\|\underline{\mathbf{Z}}\|_{f}^{2} \pm\left(\left(1 / 2\|\underline{\mathbf{Z}}\|_{f}^{2}\right)^{2}-|\operatorname{det}(\underline{\mathbf{Z}})|^{2}\right)^{1 / 2}$ sowie die Frobenius-Norm $\|\underline{\mathbf{Z}}\|_{f}=\left(\left|Z_{x x}\right|^{2}+\left|Z_{x y}\right|^{2}+\left|Z_{y x}\right|^{2}+\left|Z_{y y}\right|^{2}\right)^{1 / 2}$.

EGGERS (1982) verwendete komplexe Pseudoeigenwerte $\lambda_{1,2}=\left(Z_{x y}-Z_{y x}\right) / 2 \pm\left(\left(\left(Z_{x y}-Z_{y x}\right) / 2\right)^{2}-\operatorname{det}(\underline{\mathbf{Z}})\right)^{1 / 2}$.

BERDICHEVSKY \& DMITRIEV (1976) führten die effektiven Impedanzen

$$
Z_{\text {eff }}^{I}=\left(Z_{x y}-Z_{y x}\right) / 2 \text { und } Z_{\text {eff }}^{I I}=\sqrt{Z_{x x} Z_{y y}-Z_{x y} Z_{y x}}
$$

ein.

Bis auf die Berdichevsky-Invarianten sind die genannten Kennzahlen allerdings eher mathematischer Natur und der unmittelbaren Anschauung wenig zugänglich. Abschließend werden noch zwei weitere Kennzahlen vorgestellt, die mit den zuvor dargestellten Modellen in unmittelbarem Zusammenhang stehen.

Als Maßzahl dafür, inwieweit die Vorstellung von der einfachen zweidimensionalen Modellvorstellung (2.3) überhaupt paßt, kann die Schiefe des Impedanztensors nach SwIFT (1967) betrachtet werden :

$$
\kappa=\frac{\left|Z_{x x}+Z_{y y}\right|}{\left|Z_{x y}-Z_{y x}\right|} .
$$

Im idealen ein- oder zweidimensionalen Fall ist sie null. Die tatsächliche Abweichung kann bis zu einem gewissen Grad als Maß für die Dreidimensionalität der untersuchten Leitfähigkeitsverteilung angesehen werden. Nachfolgendes Beispiel (Abbildung 2.4) illustriert dies. Hier wurde die Schiefe $\kappa$ für das Modell V (Modellbeschreibung in Abbildung 1.5) berechnet.

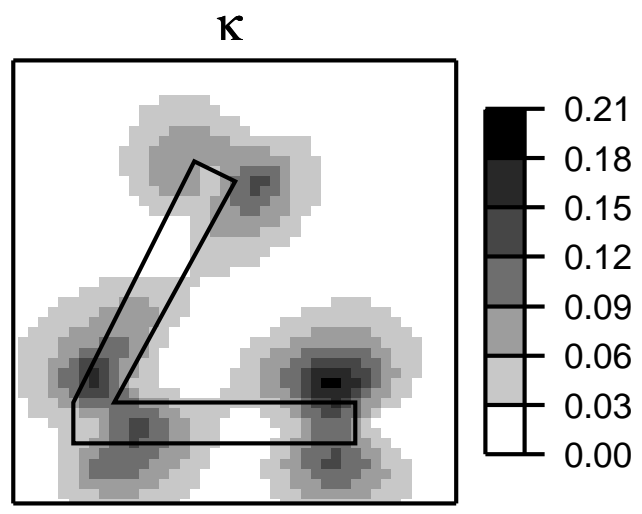

Abbildung 2.4:

Darstellung von $\kappa$ über dem Modell $\mathrm{V}$ (s. Abbildung 1.5) bei einer Periode von $85 \mathrm{~s}$.

Man erkennt, daß die Schiefe an den Endpunkten der V-förmigen Anomalie besonders groß wird und Werte von $\kappa>0.2$ erreicht. Etwa in der Mitte der beiden Seiten wird $\kappa \approx 0$ 
und zeigt an, daß die Leitfähigkeitsverteilung an dieser Stelle im Vergleich zur Skintiefe der betrachteten Frequenz zweidimensional ist.

Bemerkenswert ist, daß direkt im Winkel die Schiefe sehr kleine Werte besitzt, obgleich die Struktur hier in höchstem Grade dreidimensional ist. Der Stromfluß verläuft symmetrisch zu den beiden Seiten der Figur und spiegelt offenbar eine zweidimensionale Struktur wider.

An dieser Modellstudie wird deutlich, daß eine Interpretation der Schiefe $\kappa$ nur in eine Richtung eindeutig ist: Sofern eine zweidimensionale Struktur vorliegt, muß $\kappa \approx 0$ sein, andererseits kann $\kappa$ jedoch auch auf den Symmetrieachsen von dreidimensionalen Strukturen sehr klein werden (s. a. TING \& HoHmAnN, 1981). Sofern es mit diesen Einschränkungen überhaupt sinnvoll ist, einen Schwellenwert zur Unterscheidung von zweiund dreidimensionalen Strukturen zu bilden, sollte dieser sehr klein sein: $\kappa<0.1$. Bei Verwendung von größeren Werten (z. B. $\kappa<0.3$, SchEELKE, 1972) ist die Gefahr gegeben, eine dreidimensionale Struktur auf der Grundlage von zweidimensionalen Modellvorstellungen zu interpretieren.

Die erweiterte zweidimensionale Modellvorstellung (2.7) und (2.8) sieht zusätzlich zu einer regionalen Struktur lokale Inhomogenitäten vor, die nur im Sinne einer galvanischen Gleichstromverzerrung Einfluß auf die Messungen haben.

Es gibt verschiedene Analysemethoden des Impedanztensors, die auf diesem Modellansatz beruhen (ZHANG, 1987; BAHR, 1988; Groom \& BAILEY, 1989; ChAVE \& SMITH, 1994). Einen Überblick über verschiedene Anätze geben z. B. GROOM \& BAHR (1992). Für den Fall der Superposition einer lokalen 3D-Anomalie mit einer regionalen 2DStruktur gibt es ebenfalls eine rotationsinvariante Maßzahl, die Abweichungen von diesem Modell anzeigt.

Die phasensensitive Schiefe nach BAHR (1988) ist ${ }^{7}$ :

$$
\eta=\frac{\sqrt{\left[D_{1}, S_{2}\right]-\left[S_{1}, D_{2}\right]}}{\left|D_{2}\right|}
$$

mit den modifizierten Impedanzen

$$
\begin{array}{cr}
S_{1}=Z_{x x}+Z_{y y} & S_{2}=Z_{x y}+Z_{y x} \\
D_{1}=Z_{x x}-Z_{y y} & D_{2}=Z_{x y}-Z_{y x} .
\end{array}
$$

Im Vergleich zur Schiefe $\kappa$ werden hier die Phasen des Impedanztensors ausgewertet. Sie werden durch eine Verzerrung der elektrischen Felder nicht beeinflußt.

Zur Illustration wurde das Modell V (Abbildung 1.5) erweitert: Um die galvanische Verzerrung zu simulieren, wurden zusätzlich zu der regionalen V-förmigen Struktur mehrere kleine, gut leitende Blöcke in der obersten Schicht eingebracht.

Die Resultate der Modellstudien sind in Abbildung 2.5a und 2.5b gezeigt.

Der Vergleich der Auftragung von $\eta$ über dem Modell mit (2.5a) und ohne Störkörper (2.5b) zeigt, daß die im Vergleich zur regionalen Struktur kleinen Anomalien so gut wie

\footnotetext{
${ }^{7}[A, B]=\operatorname{Re}\{A\} \operatorname{Im}\{B\}-\operatorname{Im}\{A\} \operatorname{Re}\{B\}$
} 
keinen Einfluß auf das Verhalten von $\eta$ haben. Die flächenhaften Darstellungen weisen keine signifikanten Unterschiede auf. An den Endpunkten sowie im Bereich des Winkels liegen die Werte von $\eta$ in beiden Modellstudien zwischen 0.2 und 0.35, in der Mitte der Seiten bei $0.05-0.15$.

a) Mit lokalen Inhomogenitäten
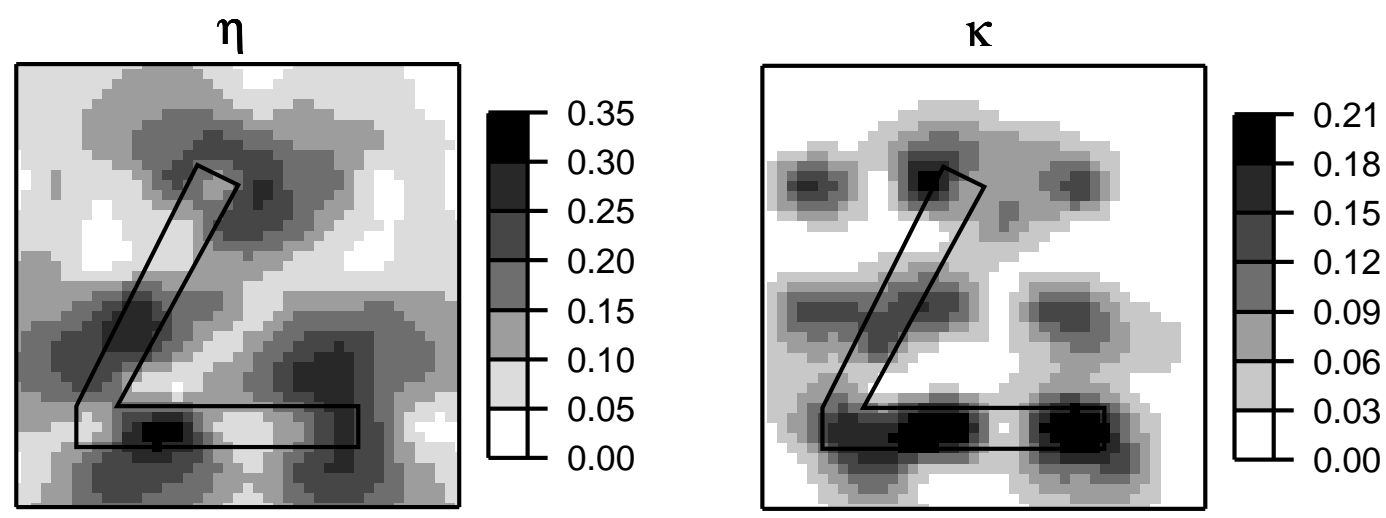

b) Ohne lokale Inhomogenitäten

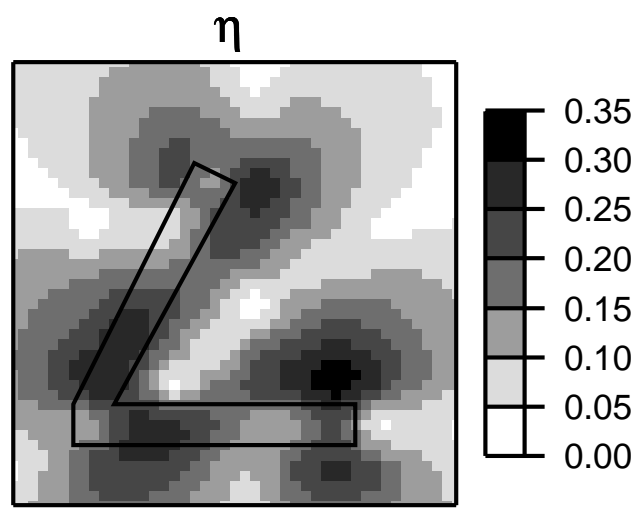

Abbildung 2.5:

Darstellung von $\eta$ und $\kappa$ für das Modell V bei $85 \mathrm{~s}$. Dabei sind in der Modellstudie a) zusätzlich in der obersten Schicht willkürlich verteilte Blöcke von $10 \times 5 \times 1 \mathrm{~km}$ Größe vorhanden. Fall b) zeigt $\eta$ für das Modell $\mathrm{V}$ ohne lokale Anomalien in der obersten Schicht (bzgl. $\kappa$ vgl. Abb. 2.4).

Im Vergleich dazu reagiert $\kappa$ sehr stark auf die lokalen Anomalien. An den Eckpunkten und im Winkel wird zwar $\kappa>0.2$. Allerdings zeigt $\kappa$ auch außerhalb der regionalen Modellstruktur lokale Maxima, die durch die kleinräumigen Anomalien hervorgerufen werden und in etwa die gleiche Amplitude aufweisen. Rückschlüsse auf die Dimensionalität der regionalen Leitfähigkeitsverteilung sind nicht mehr möglich.

Falls $\eta>0.3$ ist, kann von einer regionalen 3D-Anomalie ausgegangen werden (BAHR, 1991). Auch in diesem Fall ist jedoch zu beachten, daß umgekehrt nicht jede dreidimensionale Struktur auch als solche erkannt wird. Wie $\kappa$ besitzt auch $\eta$ im Winkel der Modellstruktur kleine Werte. 


\subsubsection{Anwendungen in der erdmagnetischen Tiefensondierung}

Aus den Elementen des Perturbationstensors $\underline{\mathbf{W}}$ lassen sich in entsprechender Weise rotationsinvariante Parameter konstruieren. Die Maßzahlen, die den im vorherigen Kapitel aus dem Impedanztensor gebildeten entsprechen, lauten:

- $r_{1}=\operatorname{Re}\left\{E_{2} / 2\right\}$

- $r_{2}=\operatorname{Im}\left\{\left(E_{2}\right) / 2\right\}$

- $r_{3}=\operatorname{Re}\left\{\left(T_{1}\right) / 2\right\}$

- $r_{4}=\operatorname{Im}\left\{\left(T_{1}\right) / 2\right\}$

- $r_{5}=\operatorname{det}(\operatorname{Re}\{\underline{\mathbf{W}}\})$

- $r_{6}=\operatorname{det}(\operatorname{Im}\{\underline{\mathbf{W}}\})$

- $r_{7}=\operatorname{Im}\{\operatorname{det}(\underline{\mathbf{W}})\}$

mit den Ausdrücken analog zu (2.14):

$$
\begin{array}{cr}
T_{1}=h_{H}+d_{D} & T_{2}=h_{D}+d_{H} \\
E_{1}=h_{H}-d_{D} & E_{2}=h_{D}-d_{H} .
\end{array}
$$

Alle Maßzahlen wurden wieder für das Modell V berechnet. In Abbildung 2.6 sind die Resultate für die Periode $85 \mathrm{~s}$ dargestellt. 

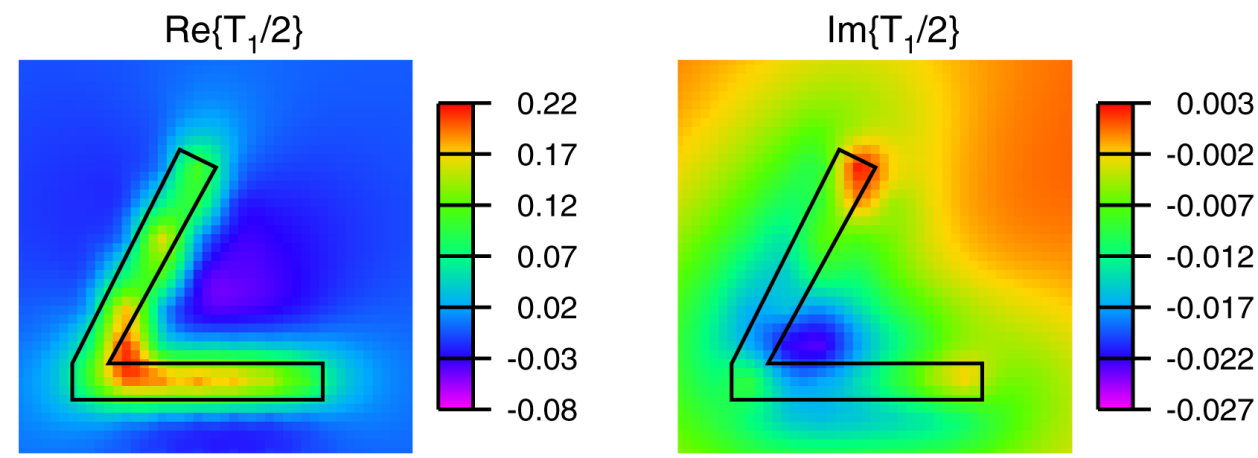

$\operatorname{Re}\left\{E_{2} / 2\right\}$

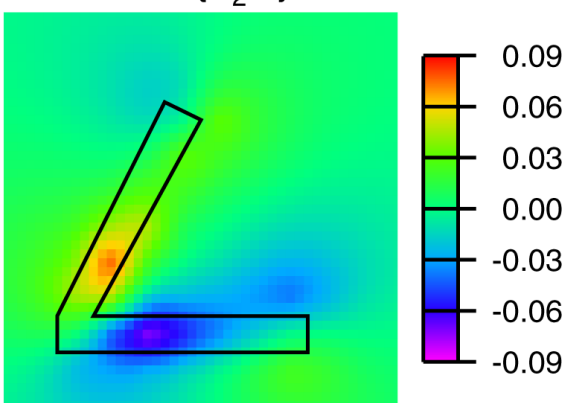

$\operatorname{Im}\left\{\mathrm{E}_{2} / 2\right\}$

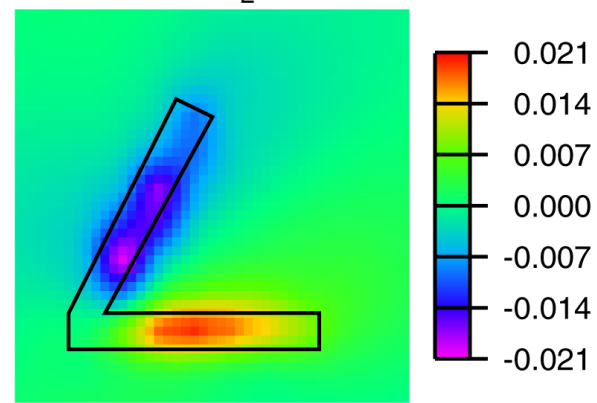

$\operatorname{det}(\operatorname{Re}\{\mathrm{W}\})$

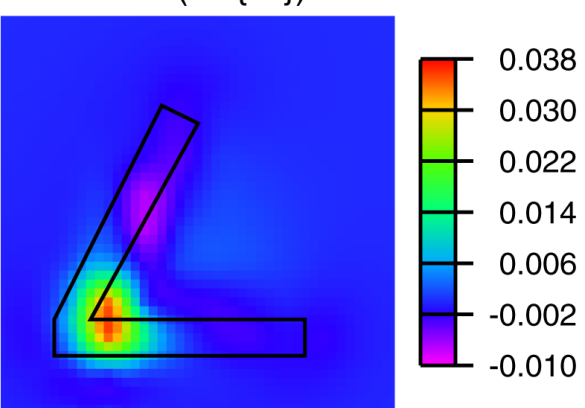

$\operatorname{det}(\operatorname{Im}\{W\})$
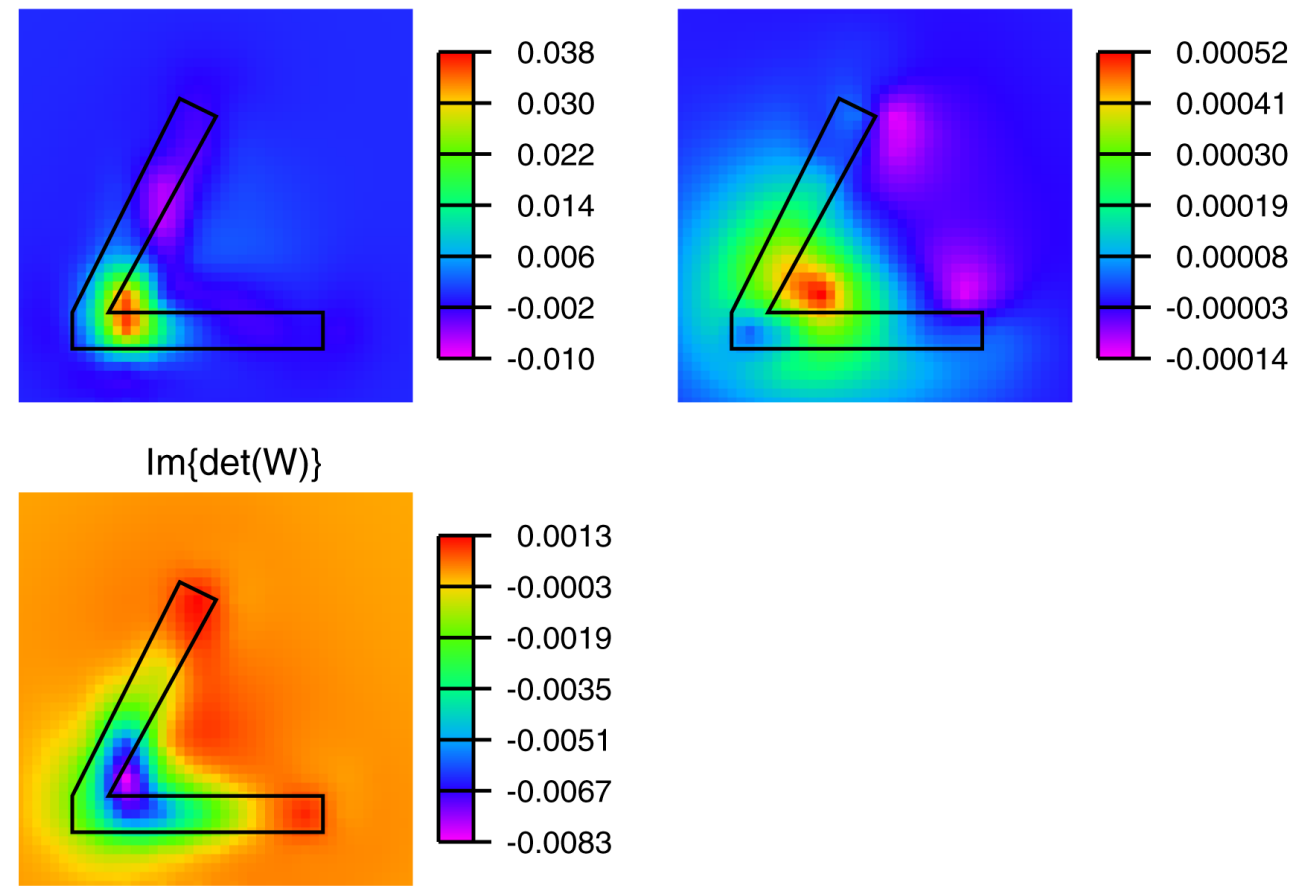

Abbildung 2.6: Darstellung von sieben rotationsinvarianten Maßzahlen des Perturbationstensors für das Modell V (Abbildung 1.5) bei $85 \mathrm{~s}$. 
Die Form der Anomalie wird durch $\operatorname{Re}\left\{T_{1} / 2\right\}$ sehr gut abgebildet. Im Vergleich zu ähnlichen Studien mit den Rotationsinvarianten des Impedanztensors (SZARKA \& MENVIELLE, 1997) sind hier insbesondere die Kanten der Struktur deutlich zu erkennen. Während jedoch die Schiefen des Impedanztensors nicht nur den Winkel der Anomalie als dreidimensionale Struktur erkennen, sondern auch die Endpunkte der jeweiligen Seiten, zeigt hier keine der magnetischen Rotationsinvarianten ein signifikant anomales Verhalten. Der Symmetrieeffekt im Winkel wird allerdings auch hier bei fast allen Parametern deutlich. Nur die Auftragung der Parameter $\operatorname{det}(\operatorname{Re}\{\underline{\mathbf{W}}\})$ und $\operatorname{Im}\{\operatorname{det}(\underline{\mathbf{W}})\}$ läßt direkt im Winkel eine lokale Anomalie erkennen. Dabei wird der Knick in der Struktur von $\operatorname{det}(\operatorname{Re}\{\underline{\mathbf{W}}\})$ noch schärfer abgebildet.

Auch das Verhalten von $\operatorname{Im}\left\{E_{2} / 2\right\}$ ist interessant: Hier werden die Bereiche des Modells besonders hervorgehoben, die näherungsweise eine zweidimensionale Leitfähigkeitsverteilung aufweisen. Sowohl der Winkel als auch die Endpunkte der Seiten haben keinen signifikanten Einfluß auf diesen Parameter.

Durch Linearkombination ist es möglich, weitere Maßzahlen zu erzeugen. Man kann eine Schiefe analog zu der des Impedanztensors bilden: $\kappa_{G}=E_{2} / T_{1}$ (z. B. SCHMUCKER \& WEIDELT, 1975) Sie wird im zweidimensionalen Fall null. Im 1D-Fall sind aber alle Elemente des Perturbationstensors null, und die Schiefe ist unbestimmt. Man kann diese Schwierigkeiten umgehen, wenn anstelle des anomalen Magnetfeldes das absolute Feld verwendet wird:

$$
\mathbf{B}=\underline{\mathbf{W B}^{n}}+\mathbf{B}^{n}=\underbrace{(\underline{\mathbf{W}}+\underline{\mathbf{I}})}_{\underline{\mathbf{W}}^{*}} \mathbf{B}^{n} .
$$

Die entsprechende Schiefe des Perturbationstensors lautet dann:

$$
\kappa_{G^{*}}=\frac{h_{D}-d_{H}}{h_{H}+d_{D}+2}
$$

(z. B. SiEMON, 1991). In ähnlicher Weise kann man

$$
\begin{aligned}
& \operatorname{det}\left(\operatorname{Re}\left\{\underline{\mathbf{W}}^{*}\right\}\right)=\operatorname{det}(\operatorname{Re}\{\underline{\mathbf{W}}\})+\operatorname{Re}\left\{T_{1}\right\}+1 \\
& \operatorname{Im}\left\{\operatorname{det}\left(\underline{\mathbf{W}}^{*}\right)\right\}=\operatorname{Im}\{\operatorname{det}(\underline{\mathbf{W}})\}+\operatorname{Im}\left\{T_{1}\right\}
\end{aligned}
$$

bilden. Von den vorgestellten Maßzahlen zeigen $\operatorname{det}(\operatorname{Re}\{\underline{\mathbf{W}}\})$ und $\operatorname{Im}\{\operatorname{det}(\underline{\mathbf{W}})$ keinen deutlichen Symmetrieeffekt und reagieren auf eine abknickende zweidimensionale Struktur empfindlicher als die magnetotellurischen Schiefen und die Schiefe (2.16). Zur Darstellung werden folgende Größen gebildet:

$$
\begin{aligned}
\kappa_{G 1} & =\sqrt{|\operatorname{det}(\operatorname{Re}\{\underline{\mathbf{W}}\})|} \\
\kappa_{G 2} & =\sqrt{\mid \operatorname{Im}\{\operatorname{det}(\underline{\mathbf{W}}) \mid} .
\end{aligned}
$$

In Abbildung 2.7 sind die Parameter $\kappa_{G 1}, \kappa_{G 2}$ und $\kappa_{G^{*}}$ zum Vergleich abgebildet. Sie wurden für das Modell V mit lokalen Anomalien bei der Periode $85 \mathrm{~s}$ berechnet. 

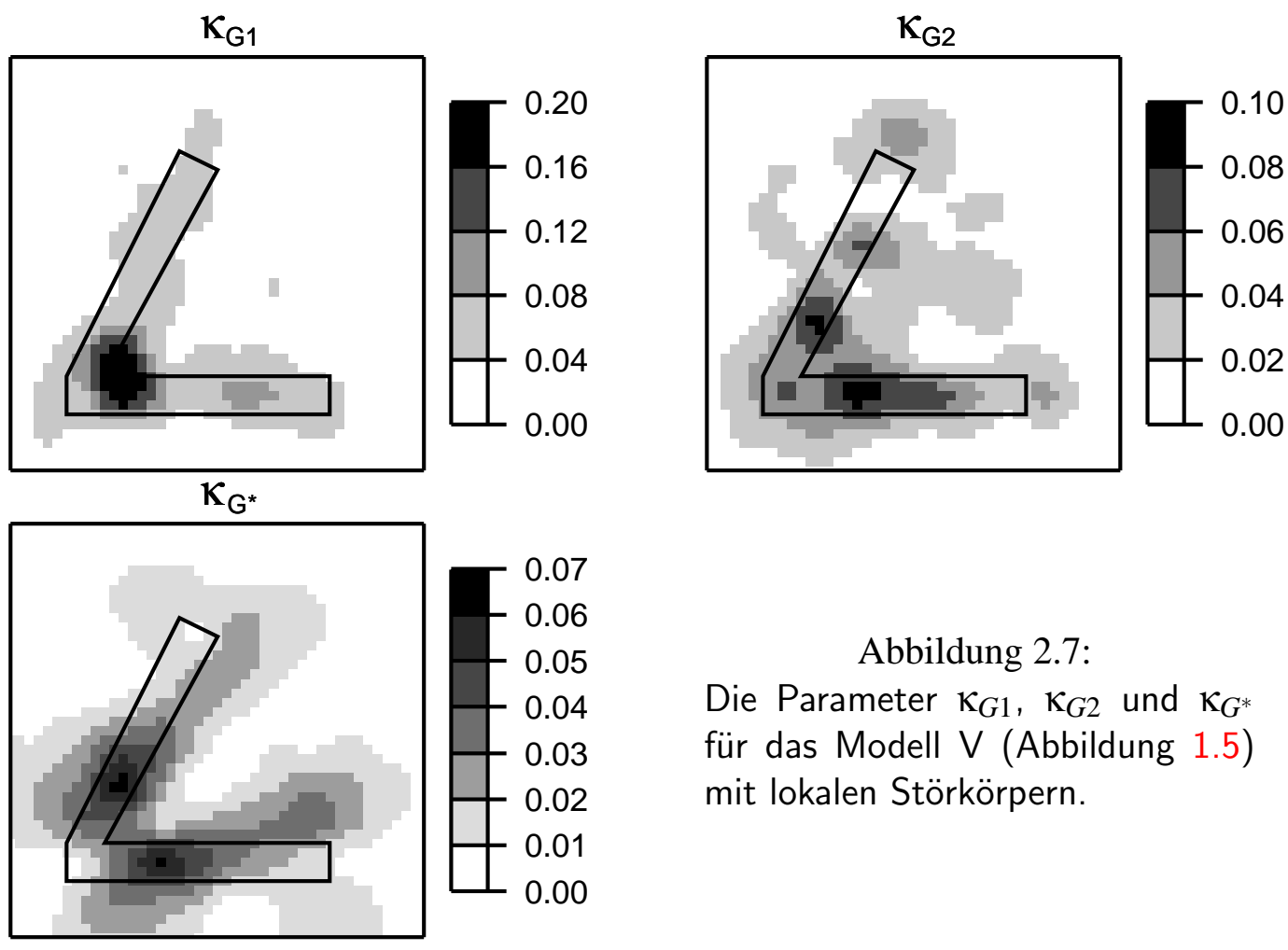

Abbildung 2.7:

Die Parameter $\kappa_{G 1}, \kappa_{G 2}$ und $\kappa_{G^{*}}$ für das Modell $\mathrm{V}$ (Abbildung 1.5) mit lokalen Störkörpern.

Beim Vergleich von $\kappa_{G 1}$ und $\kappa_{G 1}$ sowohl untereinander als auch mit den Auftragungen der entsprechenden Größen $\operatorname{det}(\operatorname{Re}\{\underline{\mathbf{W}}\})$ und $\operatorname{Im}\left\{\operatorname{det}(\underline{\mathbf{W}})\right.$ in Abbildung 2.6 fällt auf, daß $\kappa_{G 1}$ von lokalen Anomalien viel weniger beeinflußt wird als $\kappa_{G 2}$. Beide Parameter unterscheiden sich unter anderem dadurch, daß $\kappa_{G 2}$ eine Mischung aus Imaginär- und Realteilen der magnetischen Übertragungsfunktionen darstellt, während $\kappa_{G 1}$ nur aus den Realteilen gebildet wird. Möglicherweise wird der Imaginärteil der magnetischen Übertragungsfunktionen stärker durch kleinräumige Anomalien beeinflußt.

Insgesamt zeigen die durchgeführten Modellstudien, daß eine Auswertung der Rotationsinvarianten des Perturbationstensors Beiträge zur Strukturbestimmung von unbekannten Leitfähigkeitsverteilungen liefern kann.

Die Form einer Leitfähigkeitsanomalie kann bei einer flächenhaften Darstellung geeigneter Parameter (etwa $\operatorname{Re}\left\{T_{1} / 2\right\}$ ) ausgezeichnet abgebildet werden. Auch im Hinblick auf Fragestellungen, inwieweit sich Daten zweidimensional interpretieren lassen, kann die Analyse der verschiedenen Maßzahlen vorteilhaft sein. Insbesondere Symmetrieeffekte, die eine zweidimensionale Struktur vortäuschen, können sicherer als bei alleiniger Verwendung der magnetotellurischen Schiefen erkannt werden: Zur Identifizierung einer zweidimensionalen Struktur sollten sowohl $\kappa_{G 1}$ als auch $\eta$ klein sein.

Eine kombinierte Auswertung der magnetotellurischen und der magnetischen Rotationsinvarianten erscheint somit sinnvoll. 


\subsection{Rotationswinkel}

Die Interpretation magnetotellurischer Daten wird wesentlich vereinfacht, wenn es gelingt, ein Koordinatensystem zu finden, in dem der Impedanztensor die Nebendiagonalform besitzt oder aber die Charakteristika des Superpositionsmodells (2.8) aufweist. Abhängig von der zugrundeliegenden Modellvorstellung gibt es mehrere Ansätze, die es ermöglichen, einen Rotationswinkel aus dem Impedanztensor zu berechnen ${ }^{8}$.

\subsubsection{Behandlung des Impedanztensors}

- Rotationswinkel nach SwIFT

Im richtigen Koordinatensystem besitzt der Impedanztensor im idealen 2D-Fall die Nebendiagonalform. Als Drehkriterium kann somit versucht werden, die Summe der Hauptdiagonalelemente zu minimieren: $\left|Z_{x x}^{\prime}\right|^{2}+\left|Z_{y y}^{\prime}\right|^{2} \rightarrow$ Min. $^{9}$ Das führt auf die analytische Lösung (SWIFT, 1967):

$$
\alpha_{S}=\frac{1}{4} \arctan \left(\frac{2 \operatorname{Re}\left\{S_{2} D_{1}^{*}\right\}}{\left|D_{1}\right|^{2}+\left|S_{2}\right|^{2}}\right)
$$

Damit das so gefundene Extremum ein Minimum ist, muß die zweite Ableitung nach $\alpha$ positiv sein. Der berechnete Winkel ist letztendlich nur bis auf $\pi / 2$ bestimmt. In der Praxis bedeutet das, daß man allein anhand des Drehwinkels nicht entscheiden kann, ob beispielsweise die $x^{\prime}$-Richtung des neuen (rotierten) Koordinatensystems parallel oder senkrecht zum lateralen Leitfähigkeitskontrast verläuft. Um eine Zuordnung der Nebendiagonalelemente zu E- und B-Polarisation vorzunehmen, ist daher eine weitere Größe nötig, etwa das vertikale Magnetfeld, welches nur in E-Polarisation auftritt.

- Rotationswinkel nach BAHR

In der Praxis erweist sich der Swift-Winkel als recht stabil, es ist dabei jedoch oft zu beobachten, daß er über einen langen Periodenbereich annähernd konstant bleibt (z. B. Peter, 1994). Die Ursache wird aber in der Regel nicht in einer tiefreichenden regionalen 2D-Struktur zu suchen sein, die die Felder über einen sehr großen Periodenbereich beeinflußt.

Da der Swift-Winkel wie der scheinbare spezifische Widerstand nur aus den Amplituden des Impedanztensors gebildet wird, gehen die Auswirkungen einer galvanischen Verzerrung des elektrischen Feldes in die Bestimmung mit ein. Das kann

\footnotetext{
${ }^{8}$ Zur Problematik der Bestimmung von Rotationswinkeln aus Felddaten, die fehlerbehaftet und möglicherweise lokal verzerrt sind vgl. a. JONES \& GROOM (1993)

${ }^{9}$ Eine Maximierung der Hauptdiagonalelemente $\left|Z_{x y}^{\prime}\right|^{2}+\left|Z_{y x}^{\prime}\right|^{2} \rightarrow$ Max. führt auf die gleiche Lösung (SCHEELKE, 1972).
} 
zur Folge haben, daß kleinräumige Anomalien den Swift-Winkel über einen weiten Periodenbereich bestimmen und die regionale Streichrichtung überdeckt wird.

Da die Phasen der Impedanz nicht durch die lokalen Störkörper beeinflußt werden, bietet es sich an, ein Drehkriterium zu benutzten, welches auf der Phaseninformation des Impedanztensors beruht.

Man geht von dem Superpositionsmodell (2.8) aus. Im Koordinatensystem des regionalen Streichens müssen die Phasen in den beiden Spalten von $\underline{\mathbf{Z}}$ jeweils gleich sein: $\operatorname{Re}\left\{Z_{x x}^{\prime}\right\} / \operatorname{Re}\left\{Z_{y x}^{\prime}\right\}=\operatorname{Im}\left\{Z_{x x}^{\prime}\right\} / \operatorname{Im}\left\{Z_{y x}^{\prime}\right\}$ und $\operatorname{Re}\left\{Z_{x y}^{\prime}\right\} / \operatorname{Re}\left\{Z_{y y}^{\prime}\right\}=\operatorname{Im}\left\{Z_{x y}^{\prime}\right\} / \operatorname{Im}\left\{Z_{y y}^{\prime}\right\}$.

Aus dieser Forderung ergibt sich eine analytische Lösung für den Drehwinkel ${ }^{10}$ (BAHR, 1988):

$$
\alpha_{B}=\frac{1}{2} \arctan \left(\frac{\left[S_{1}, S_{2}\right]-\left[D_{1}, D_{2}\right]}{\left[S_{1}, D_{1}\right]+\left[S_{2}, D_{2}\right]}\right) .
$$

Dieser Ansatz liefert sinnvolle Ergebnisse, solange $\eta<0.1$ ist. Dann kann davon ausgegangen werden, daß die Modellannahme eines galvanischen Störkörpers und einer regionalen 2D-Struktur in guter Näherung zutrifft. Für kleinere Abweichungen $(\eta<0.3)$ von dieser Vorstellung kann der Ansatz (2.8) erweitert werden (BAHR, 1991). Es wird zusätzlich eine geringe Phasendifferenz $\delta$ innerhalb der Spalten zugelassen:

$$
\underline{\mathbf{Z}}=\left(\begin{array}{cc}
C_{x y} Z_{y x} e^{i \delta} & C_{x x} Z_{x y} \\
C_{y y} Z_{y x} & C_{y x} Z_{x y} e^{-i \delta}
\end{array}\right) .
$$

Als Drehkriterium wird gefordert, daß im Koordinatensystem des regionalen Streichens der Betrag der Phasendifferenz in der linken Spalte $\arg \left\{Z_{x x}^{\prime}\right\}-\arg \left\{Z_{y x}^{\prime}\right\}=\delta$ derjenigen der rechten Spalte $(-\delta)$ entspricht. So wird eine Bevorzugung einer bestimmten Spalte vermieden. Der Ansatz (2.8) ist als Spezialfall $\delta=0$ enthalten. Auch in diesem Fall kann der Drehwinkel in analytischer Form angegeben werden (BAHR, 1991).

- Extremwerte der Phasendifferenz

Ist ausschließlich die Phase des Impedanztensors von Interesse, ist es sinnvoll, sie in einem Koordinatensystem zu betrachten, in dem eine maximale Aufspaltung oder auch eine minimale Abweichung der Phasen der Nebendiagonalelemente auftritt:

$$
\left|\phi_{x y}-\phi_{y x}\right| \rightarrow \text { Extr. }
$$

Dieses Drehkriterium führt auf einen Winkel $\alpha_{P}$. Bei einer reinen zweidimensionalen Struktur ist die Phasenaufspaltung in der E- und B-Polarisation maximal. In diesem Fall ist $\eta=0$, und $\alpha_{P}$ entspricht dem phasensensitiven Rotationswinkel $\alpha_{B}$ (2.21).

${ }^{10}$ Aus den obigen Bedingungen erhält man zwei Winkel für die beiden Spalten, die sich im Idealfall $\eta=0$ um $90^{\circ}$ unterscheiden. 


\subsubsection{Behandlung des Perturbationstensors}

Auch bei der Bestimmung eines Rotationswinkels aus dem Perturbationstensor sind verschiedene Ansätze denkbar. Dabei ist zu beachten, daß im Koordinatensystem einer regionalen 2D-Struktur nur ein Element des Perturbationstensors von null verschieden ist. Das hat zur Konsequenz, daß der gefundene Rotationswinkel eindeutig die Streichrichtung angibt, je nachdem, welches Hauptdiagonalelement nach der Rotation übrigbleibt. FLUCHE (1983) minimierte die Nebendiagonalelemente $\left|h_{D}^{\prime}\right|^{2}+\left|d_{H}^{\prime}\right|^{2} \rightarrow$ Min. und erhielt für den Drehwinkel:

$$
\beta_{F}=\frac{1}{4} \arctan \left(\frac{2 \operatorname{Re}\left\{E_{1} T_{2}^{*}\right\}}{\left|E_{1}\right|^{2}+\left|T_{2}\right|^{2}}\right)
$$

Ebenso können die vertikalen Übertragungsfunktionen für eine Drehwinkelbestimmung verwendet werden, z. B. $\left|z_{H}^{\prime}\right|^{2} \rightarrow$ Min. (PETER, 1994). Bei der Bestimmung einer regionalen Streichrichtung aus dem Perturbationstensor ist jedoch immer zu beachten, daß der gefundene Winkel nur dann die tatsächlichen Verhältnisse am Meßort wiedergibt, wenn die Normalfelder an einer Referenzstation ohne laterale Leitfähigkeitskontraste gemessen wurden.

\subsection{Beiträge zur Analyse des Perturbationstensors}

\subsubsection{Zur Wahl der Referenzstation}

Es wurde bereits mehrfach erwähnt, daß die Güte der Referenzstation bei der Interpretation der magnetischen Übertragunsfunktionen von entscheidender Bedeutung ist. Neben einer guten Datenqualität spielen bei der Auswahl der Referenzstation mehrere Überlegungen eine Rolle.

Um eine Vergleichbarkeit der Ergebnisse mit anderen, z. B. früher gewonnenen Meßdaten zu ermöglichen, ist es sinnvoll, alle Felder auf die Referenzdaten eines permanent registrierenden Observatoriums zu beziehen. Im Idealfall liegt die Referenzstation auf einem annähernd eindimensionalen Untergrund. Das bedeutet, daß alle Feldgrößen in der Umgebung des Observatoriums möglichst geringe Gradienten sowohl im räumlichen Feldverhalten (laterale Homogenität) als auch im Frequenzgang (vertikale Homogenität) besitzen. In der Praxis sind diese Voraussetzungen in der Regel nicht erfüllt. Im Verlauf einer Meßkampagne, die mehrere Stationen in einem Array umfaßt, kann es für spezielle Untersuchungen außerdem günstig sein, als Referenzstation eine Station des Arrays zu wählen.

Aus diesen Gründen wird es im Verlauf der Datenanalyse und Interpretation möglicherweise notwendig sein, die Referenzstation zu wechseln. Das ist möglich, wenn die beiden Referenzstationen, die ausgetauscht werden sollen, zeitgleich registriert haben. Das heißt, es müssen Übertragungsfunktionen zwischen alter und neuer Referenzstation vorliegen. Es wird im folgenden gezeigt, daß es nicht notwendig ist, daß die Feldstation selbst zeitgleich mit der neuen Referenzstation aufgezeichnet hat. 
Gegeben seien die Übertragungsfunktionen einer Station $A$ bezogen auf eine Station $B$ :

$$
\mathbf{B}^{A}-\mathbf{B}^{B}=\underline{\mathbf{W}}^{A, B} \mathbf{B}^{B} .
$$

Um die Daten der Station $A$ auf eine Station $C$ zu beziehen, müssen die Übertragungsfunktionen zwischen $B$ und $C$ bekannt $\operatorname{sein}^{11}$ :

$$
\mathbf{B}^{B}-\mathbf{B}^{C}=\underline{\mathbf{W}}^{B, C} \mathbf{B}^{C} .
$$

Der gewünschte Ausdruck für die Übertragungsfunktionen zwischen $A$ und $C$ kann dann leicht berechnet werden:

$$
\mathbf{B}^{A}-\mathbf{B}^{C}=\underbrace{\left(\underline{\mathbf{W}}^{A, B} \underline{\mathbf{W}}^{B, C}+\underline{\mathbf{W}}^{A, B}+\underline{\mathbf{W}}^{B, C}\right)}_{\underline{\mathbf{W}}^{A, C}} \mathbf{B}^{C} .
$$

Explizite Ausdrücke für die einzelnen Elemente von $\underline{\mathbf{W}}^{A, C}$ sind im Anhang A.2 aufgeführt.

Ausgehend von obigen Überlegungen soll nun anhand von zwei Beispielen die entstehende Problematik verdeutlicht werden, sobald eine Referenzstation keinen eindimensionalen Untergrund aufweist.

\section{Beispiel 1}

An der Station $A$ liege eine zweidimensionale Anomalie mit Streichrichtung in $x$-Richtung vor. Bezogen auf eine eindimensionale Referenzstation $B$ besitzt der Perturbationstensor im richtigen Koordinatensystem nur ein Element

$$
\underline{\mathbf{W}}^{A, B}=\left(\begin{array}{cc}
0 & 0 \\
0 & d_{D}^{A, B}
\end{array}\right)
$$

und spiegelt die tatsächliche Struktur des Untergrundes an der Station $A$ wider.

Sei nun eine weitere Station $C$ (ebenfalls bezogen auf $B$ ) gegeben. Dort liegt eine 2DAnomalie mit Streichrichtung in $y$-Richtung vor, so daß

$$
\underline{\mathbf{W}}^{C, B}=\left(\begin{array}{cc}
h_{H}^{C, B} & 0 \\
0 & 0
\end{array}\right) \quad \text { ist. }
$$

${ }^{11}$ Möglicherweise liegen nur die Übertragungsfunktionen der Station $C$ bezogen auf $B$ vor:

$$
\mathbf{B}^{C}-\mathbf{B}^{B}=\underline{\mathbf{W}}^{C, B} \mathbf{B}^{B} .
$$

Dann ist es notwendig, Feld- und Referenzstation zu vertauschen. Es ergibt sich:

$$
\mathbf{B}^{B}-\mathbf{B}^{C}=\underbrace{-\underline{\mathbf{W}}^{C, B}\left(\underline{\mathbf{W}}^{C, B}+\underline{\mathbf{I}}\right)^{-1}}_{\underline{\mathbf{W}}^{B, C}} \mathbf{B}^{C} .
$$


Berechnet man nun die Übertragungsfunktionen der Station $A$ bezogen auf die Station $C$ mittels (2.23), so erhält man:

$$
\underline{\mathbf{W}}^{A, C}=\left(\begin{array}{cc}
\frac{-h_{H}^{C, B}}{1+h_{H}^{C, B}} & 0 \\
0 & d_{D}^{A, B}
\end{array}\right) .
$$

Vergleicht man den erhaltenen Ausdruck mit (2.24), so sieht man, daß durch den Wechsel der Referenzstation von $B$ nach $C$ ein zusätzliches Element $h_{H}^{A, C}$ entsteht. Dieses wird jedoch nicht durch eine Leitfähigkeitsanomalie bei $A$ erzeugt, sondern allein durch den Einfluß der neuen, auf einem nicht eindimensionalen Untergrund befindlichen Referenzstation. Eine Auswertung der magnetischen Übertragungsfunktionen $\underline{\mathbf{W}}^{A, C}$ allein im Hinblick auf die lokale Leitfähigkeitsverteilung bei Station $A$ würde in diesem Fall zu einer Fehlinterpretation führen.

\section{Beispiel 2}

Gegeben seien mehrere Stationen $A_{i}$ in Form eines Arrays, die auf eine gemeinsame Referenzstation $B$ bezogen sind. Im Gegensatz zu obigem Beispiel können die Stationen $A_{i}$ durch eine beliebige Leitfähigkeitsverteilung beeinflußt sein, die Perturbationstensoren sind also voll besetzt:

$$
\underline{\mathbf{W}}^{A_{i}, B}=\left(\begin{array}{cc}
h_{H}^{A_{i}, B} & h_{D}^{A_{i}, B} \\
d_{H}^{A_{i}, B} & d_{D}^{A_{i}, B}
\end{array}\right) .
$$

Die Bezugsstation wird gewechselt, neue Referenz soll wieder Station $C$ aus Beispiel 1 sein (Gleichung 2.25).

Man erhält so für die Perturbationstensoren der Stationen $A_{i}$, bezogen auf die neue Referenz $C$ :

$$
\underline{\mathbf{W}}^{A_{i}, C}=\left(\begin{array}{cc}
\frac{h_{H}^{A_{i}, B}-h_{H}^{C, B}}{1+h_{H}^{C, B}} & h_{D}^{A_{i}, B} \\
\frac{d_{H}^{A_{i}, B}}{1+h_{H}^{C, B}} & d_{D}^{A_{i}, B}
\end{array}\right) .
$$

Beim Vergleich von $\underline{\mathbf{W}}^{A_{i}, B}$ mit $\underline{\mathbf{W}}^{A_{i}, C}$ wird ersichtlich, daß die einzelnen Übertragungsfunktionen durch den Wechsel der Bezugstation unterschiedlich beeinflußt werden. Während $h_{D}^{A_{i}, C}=h_{D}^{A_{i}, B}$ und $d_{D}^{A_{i}, C}=d_{D}^{A_{i}, B}$ ist, taucht in den Ausdrücken für $h_{H}^{A_{i}, C}$ und $d_{H}^{A_{i}, C}$ zusätzlich das Element $h_{H}^{C, B}$ auf. Dieses beeinflußt alle Stationen des Arrays gleichermaßen. Das bedeutet, daß bei flächenhaften Auftragungen einzelner Elemente von $\underline{\mathbf{W}}^{A_{i}, B}$ oder $\underline{\mathbf{W}}^{A_{i}, C}$ die Abbildungen lateraler Leitfähigkeitskontraste erhalten bleiben. Bei Darstellungen von Parametern, die mehrere Übertragungsfunktionen kombinieren, etwa Perturbationspfeilen, gilt dies jedoch nicht mehr. 
$\mathrm{p}$

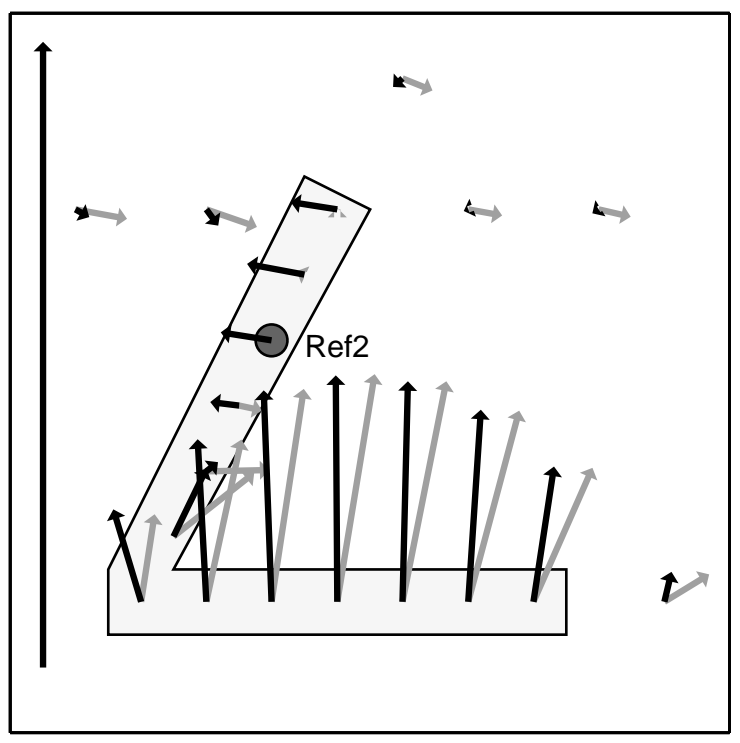

q

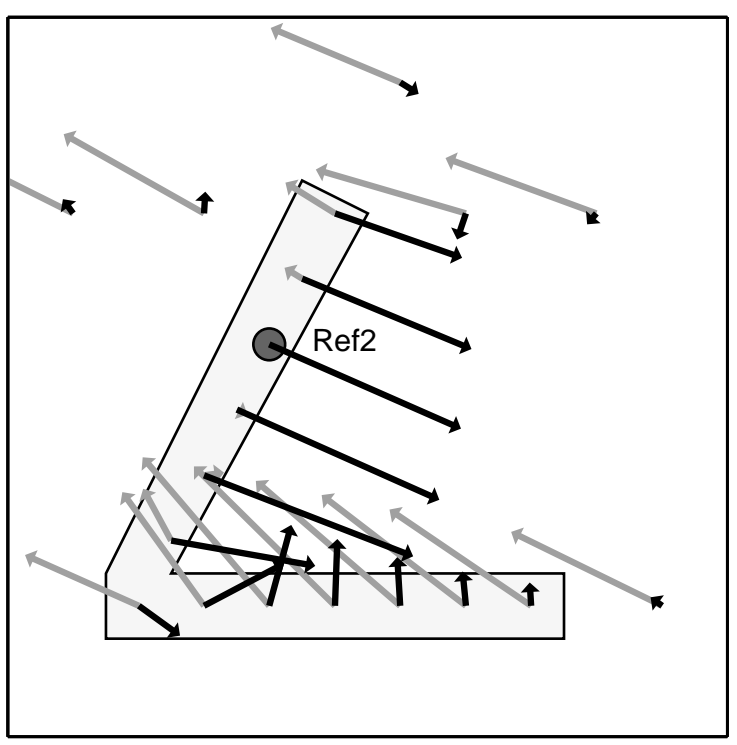

Abbildung 2.8: Darstellung der Realteile der Perturbationspfeile (vgl. Kap. 1.7) für das Modell V (Abbildung 1.5) bei $85 \mathrm{~s}$. Schwarz dargestellte Pfeile sind auf eine Referenzstation mit einer 1D - Leitfähigkeitsverteilung bezogen, grau dargestellte Pfeile sind auf die eingezeichnete Station Ref2 bezogen.

Die folgende Modellstudie verdeutlicht das. Für das Modell V (Abbildung 1.5) wurden die Perturbationspfeile bei $85 \mathrm{~s}$ berechnet und die Realpfeile jeweils bezogen auf zwei unterschiedliche Referenzstationen flächenhaft dargestellt (Abbildung 2.8).

Die schwarzen Pfeile kennzeichnen die Realteile der Perturbationspfeile, die auf eine eindimensionale Referenzstation weit außerhalb der Anomalie bezogen sind. Man erkennt, daß die Form des Körpres gut abgebildet wird. Die Perturbationspfeile stehen senkrecht auf den Leitfähigkeitskontrasten und verschwinden außerhalb der Struktur.

Ein völlig anderes Bild ergibt sich, wenn man alle Felder auf eine Station nahe eines Leitfähigkeitskontrastes bezieht. Im Modellbeispiel wurde eine Station gewählt, die sich direkt auf der Anomalie befindet (Ref2). Die auf diese Station bezogenen (grau gefärbten) Perturbationspfeile stehen nicht senkrecht auf der unteren Seite der Struktur. Die obere Seite wird überhaupt nicht abgebildet, da hier kein anomales Feld bezogen auf Ref2 vorliegt. Außerhalb der Struktur wird dagegen ein anomales Feld angezeigt.

Die vorstehenden Überlegungen und Modellrechnungen machen deutlich, daß es von entscheidender Bedeutung ist, eine Referenzstation zu wählen, die eine annähernd eindimensionale Leitfähigkeitsverteilung besitzt. Andernfalls können in den magnetischen Übertragungsfunktionen einer Meßstation Effekte auftreten, die zum Teil oder ausschließlich von der Referenzstation herrühren. 


\subsubsection{Vorschlag zur Simulation des magnetischen Normalfeldes}

Die Felddaten beliebig vieler Stationen $A_{i}$ liegen in Form von Übertragungsfunktionen vor, die auf eine gemeinsame Referenzstation $R$ bezogen sind. Für das im folgenden vorgeschlagene Verfahren spielt die Anzahl der Feldstationen keine Rolle, so daß von einer einzelnen Station $A$ ausgegangen wird:

$$
\mathbf{B}^{A}-\mathbf{B}^{R}=\underline{\mathbf{W}}^{A, R} \mathbf{B}^{R} .
$$

In der Praxis wird es die Regel sein, daß die gewählte Referenzstation $R$ keine eindimensionale Leitfähigkeitsverteilung aufweist.

Ziel soll es sein, die gewonnenen Meßdaten der Station $A$ von jeglichem Einfluß der Bezugsstation zu befreien, die Daten also in diesem Sinne auf eine „echte“ 1D-Station zu beziehen. Sie können dann unmittelbar interpretiert werden, da alle anomalen Effekte ihre Ursache ausschließlich in Leitfähigkeitskontrasten am Ort der Feldstation $A$ haben. Um dies zu erreichen, wird folgendes Verfahren vorschlagen:

Zunächst wird ein Modell entworfen, welches die lokalen Daten der bisherigen Referenzstation $R$ erklärt. Das sind z. B. das vertikale Magnetfeld oder die magnetotellurischen Parameter. Das geschieht in diesem Fall mit Hilfe dreidimensionaler Vorwärtsrechnungen $^{12}$.

Innerhalb dieser Modellrechnungen können die Magnetfelder an der modellierten Referenzstation $B^{M}$ auf einen unendlich weit entfernten Bezugspunkt, bzw. auf die Felder einer eindimensionalen Normalstation $B^{n}$ (im Modell also weit außerhalb jeglicher horizontaler Leitfähigkeitskontraste) bezogen werden:

$$
\mathbf{B}^{M}-\mathbf{B}^{n}=\underline{\mathbf{W}}^{M, n} \mathbf{B}^{n} .
$$

Die sich dabei ergebenden Übertragungsfunktionen $\underline{\mathbf{W}}^{M, n}$ sind rein synthetischer Natur. Das Modell optimiert man so lange, bis die lokalen Daten der bisherigen Referenzstation $R$ hinreichend gut erklärt werden. Wenn dies erreicht ist, kann man davon ausgehen, daß die modellierten Übertragungsfunktionen $\underline{\mathbf{W}}^{M, n}$ den Übertragungsfunktionen der Referenzstation $R$ entsprächen, wenn man deren Felder auf eine echte 1D-Station beziehen würde. Die zur Berechnung von $\underline{\mathbf{W}}^{A, n}$ fehlenden Übertragungsfunktionen $\underline{\mathbf{W}}^{R, n}$ können also durch $\underline{\mathbf{W}}^{M, n}$ ersetzt werden.

Unter Verwendung von (2.23) kann nun der letzte Schritt vollzogen werden. Die Felddaten der Station $A$ werden auf die synthetische Normalstation bezogen:

$$
\mathbf{B}^{A}-\mathbf{B}^{n}=\underbrace{\left(\left(\underline{\mathbf{W}}^{A, R}+\underline{\mathbf{I}}\right)\left(\underline{\mathbf{W}}^{R, n}+\underline{\mathbf{I}}\right)\right)}_{\underline{\mathbf{W}}^{A, n}} \mathbf{B}^{n} .
$$

\footnotetext{
12 SCHMUCKER (1993) schlug für den 2D-Fall ein iteratives Verfahren vor, welches die Berechnung von magnetischen Übertragungsfunktionen gestattete, die auf eine unendlich weit entfernte Bezugstation waren.
} 
Existiert beispielsweise ein Modell für ein permanent registrierendes Observatorium, so könnten sämliche zukünftige Messungen problemlos auf eine 1D-Station bezogen werden.

Für die Anwendung dieses Verfahrens ist es notwendig, daß das Modell der Leitfähigkeitsverteilung unter der Referenzstation von sehr guter Qualität ist.

\subsubsection{Einfluß der Verzerrung auf das magnetische Horizontalfeld}

Die Verzerrung des magnetischen Feldes wurde in der Vergangenheit mehrfach anhand der Vertikalkomponenten untersucht (ZHANG et al., 1993; RITTER, 1996). Im folgenden soll der Einfluß der Verzerrung auf das horizontale Magnetfeld ermittelt werden. Gegeben sei eine regionale Struktur mit den zugehörigen Feldgrößen:

$$
\mathbf{E}^{R}=\underline{\mathbf{Z}}^{R} \mathbf{B}^{R}
$$

Am Meßort sei außerdem eine kleinräumige Inhomogenität vorhanden, die eine galvanische Verzerrung des elektrischen und magnetischen Feldes bewirken soll. Das gemessene elektrische und magnetische Feld wird dann beschrieben durch:

$$
\mathbf{E}=\underline{\mathbf{C E}}^{R} \quad \text { und } \quad \mathbf{B}=\mathbf{B}^{R}+\underline{\mathbf{D}} \mathbf{E}^{R} .
$$

Im Ausdruck für das magnetische Feld beschreibt $\mathbf{B}^{R}$ ein magnetisches Feld, das durch Induktion in der regionalen Struktur entsteht und $\underline{\mathbf{D}} \mathbf{E}^{R}$ ein magnetisches Feld, das durch Verzerrung hervorgerufen wird. Bezogen auf ein Referenzfeld $\mathbf{B}^{n}$ erhält man:

$$
\mathbf{B}-\mathbf{B}^{n}=\left(\underline{\mathbf{D}} \underline{\mathbf{Z}}^{R}+\underline{\mathbf{I}}\right) \mathbf{B}^{R}-\mathbf{B}^{n}=\underline{\mathbf{W}}^{A, n} \mathbf{B}^{n} .
$$

Um den Einfluß der magnetischen Verzerrung zu analysieren, ist es wünschenswert, die Verzerrungsmatrix $\underline{\mathbf{D}}$ zu isolieren.

Der einfachste Fall besteht aus einem geschichtetem Halbraum mit einem lokalem Störkörper. Dann besteht kein Unterschied zwischen regionalem und normalem Feld, und in Gleichung (2.30) kann $\mathbf{B}^{R}$ durch $\mathbf{B}^{n}$ ersetzt werden. Man erhält die einfache Beziehung:

$$
\underline{\mathbf{W}}^{A, n}=\underline{\mathbf{D}} \underline{\mathbf{Z}}^{n} .
$$

Der gemessene Perturbationstensor besteht aus der reellen Matrix $\underline{\mathbf{D}}$ multipliziert mit dem Impedanztensor der normalen Struktur. Das bedeutet analog zur Struktur des verzerrten Impedanztensors (2.7), daß die Phasen aller Elemente des Perturbationstensors identisch sind und darüberhinaus auch mit den Phasen der regionalen Impedanz übereinstimmen. Zur Verdeutlichung soll folgendes Modellbeispiel dienen. Das Modell besteht aus einem geschichteten Halbraum mit einer oberflächennahen kleinräumigen Leitfähigkeitsanomalie (Modellbeschreibung in Abbildung 1.8). In Abbildung 2.9 werden die Phasen der Impedanz mit den Phasen aller Elemente des Perturbationstensors an einer Station in der Nähe der lokalen Anomalie verglichen. 


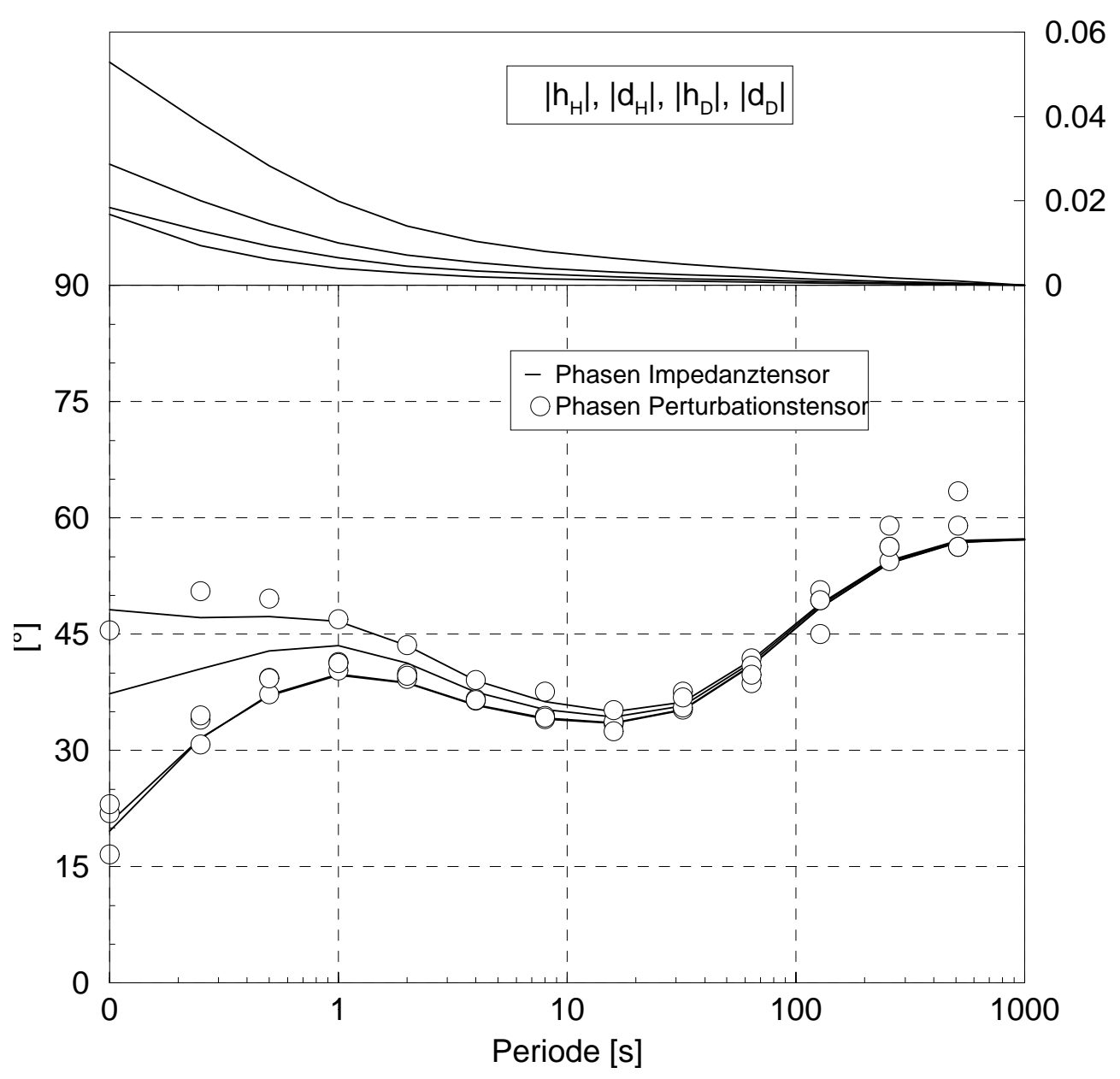

Abbildung 2.9: Vergleich der Phasen des Impedanztensors und des Perturbationstensors an einem Meßpunkt über einem geschichteten Halbraum in der Nähe eines lokalen Störkörpers. Zur Modellbeschreibung siehe Abbildung 1.8.

Die Phasen der Impedanz laufen etwa ab einer Periode von 10 s annähernd deckungsgleich. Die Phasen des Perturbationstensors zeigen eine größere Schwankungsbreite bei Perioden $>100$ s. Man erkennt aber dennoch gut, wie sie dort den Phasenverläufen der normalen Impedanz folgen. Anhand der Grafik kann abgeschätzt werden, daß der Perturbationstensor ab etwa $10 \mathrm{~s}$ die Form (2.31) annimmt.

Im oberen Teil der Abbildung sind zusätzlich die Frequenzgänge der Beträge der einzelnen Übertragungsfunktionen gezeigt. Ab einer Periode von $10 \mathrm{~s}$ werden sie sehr klein, was die unsichere Bestimmung der Phase bei langen Perioden erklärt. Der Vergleich mit den Phasengängen zeigt, daß die Anomalie nur bei kleineren Perioden bis etwa $10 \mathrm{~s}$ einen nennenswerten induktiven Einfluß hat.

Eine nicht eindimensionale regionale Struktur läßt sich berücksichtigen, indem alle Felder auf das entsprechende regionale Feld bezogen werden. In Gleichung (2.30) kann dann das normale Feld $\mathbf{B}^{n}$ durch das regionale Feld $B^{R}$ ersetzt werden. Im richtigen Koordi- 
natensystem nimmt der Perturbationstensor eine Gleichung (2.8) entsprechende Gestalt an:

$$
\underline{\mathbf{W}}^{A, R}=\underline{\mathbf{D}}\left(\begin{array}{cc}
0 & Z_{x y}^{R} \\
Z_{y x}^{R} & 0
\end{array}\right) .
$$

Die Phasen sind in diesem Fall spaltenweise gleich und die entsprechenden Analysemethoden des Impedanztensors können analog angewendet werden. Als Maß dafür, inwieweit die Modellvorstellung (2.32) der Realität entspricht, kann man entsprechend Gleichung (2.13) eine phasensensitive Schiefe des Perturbationstensors definieren:

$$
\eta_{G}=\frac{\sqrt{\left[E_{1}, T_{2}\right]-\left[T_{1}, E_{2}\right]}}{\left|E_{2}\right|} .
$$

Die Streichrichtung der regionalen Struktur wird aus dem Perturbationstensor mit

$$
\beta_{G}=\frac{1}{2} \arctan \left(\frac{\left[E_{1}, T_{2}\right]-\left[T_{1}, E_{2}\right]}{\left[T_{1}, E_{1}\right]+\left[T_{2}, E_{2}\right]}\right)
$$

oder gegebenenfalls mit dem erweiterten Ansatz (2.21) bestimmt.

Die Ermittlung der Streichrichtung einer 2D-Sruktur mit Hilfe magnetischer Übertragungsfunktionen soll anhand einer Modellstudie getestet werden. Zu diesem Zweck wird das in Abbildung 1.8 beschriebene Modell erweitert, indem zusätzlich eine großräumige 2D-Struktur eingefügt wird. Die Streichrichtung entspricht der $x$-Richtung, der Streichwinkel dieser Struktur ist also $0^{\circ}$. Der detaillierte Aufbau des Modells ist in Abbildung 2.10 skizziert.
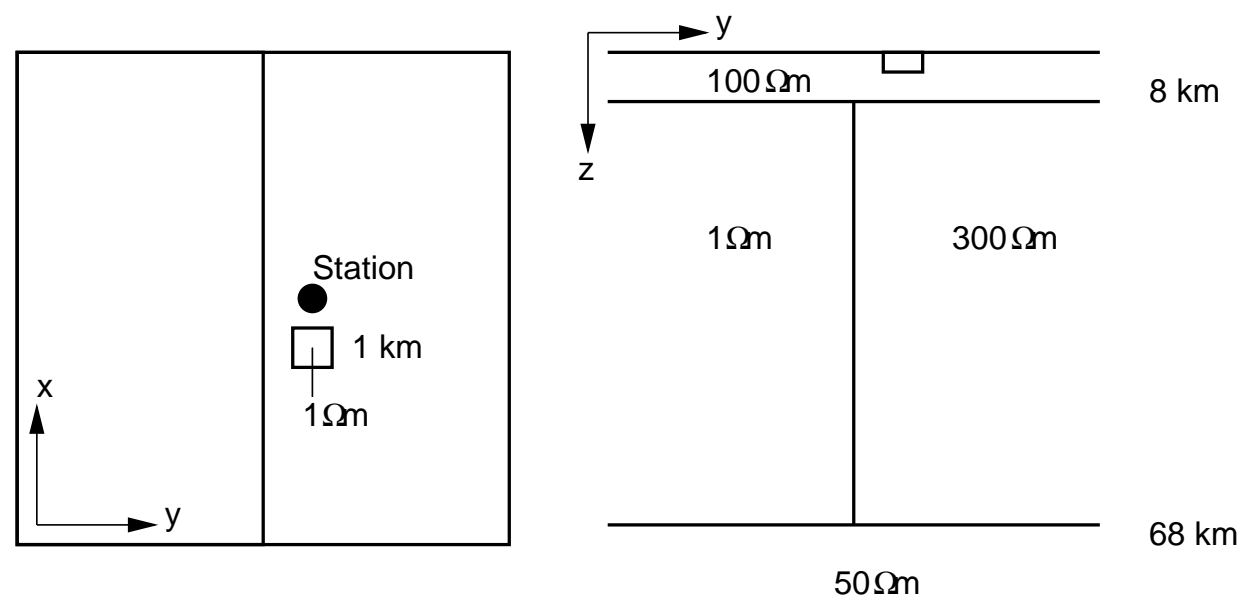

Abbildung 2.10: Aufbau des Modells N.

Die Referenzstation wird in großer Entfernung vom Störkörper, jedoch in der Nähe des Leitfähigkeitskontrastes plaziert, um das Verhalten der Feldgrößen nur unter dem Einfluß der regionalen Struktur zu simulieren. Berechnet wird der Rotationswinkel $\beta_{G}$ und die Schiefe $\eta_{G}$ (Abbildung 2.11). 


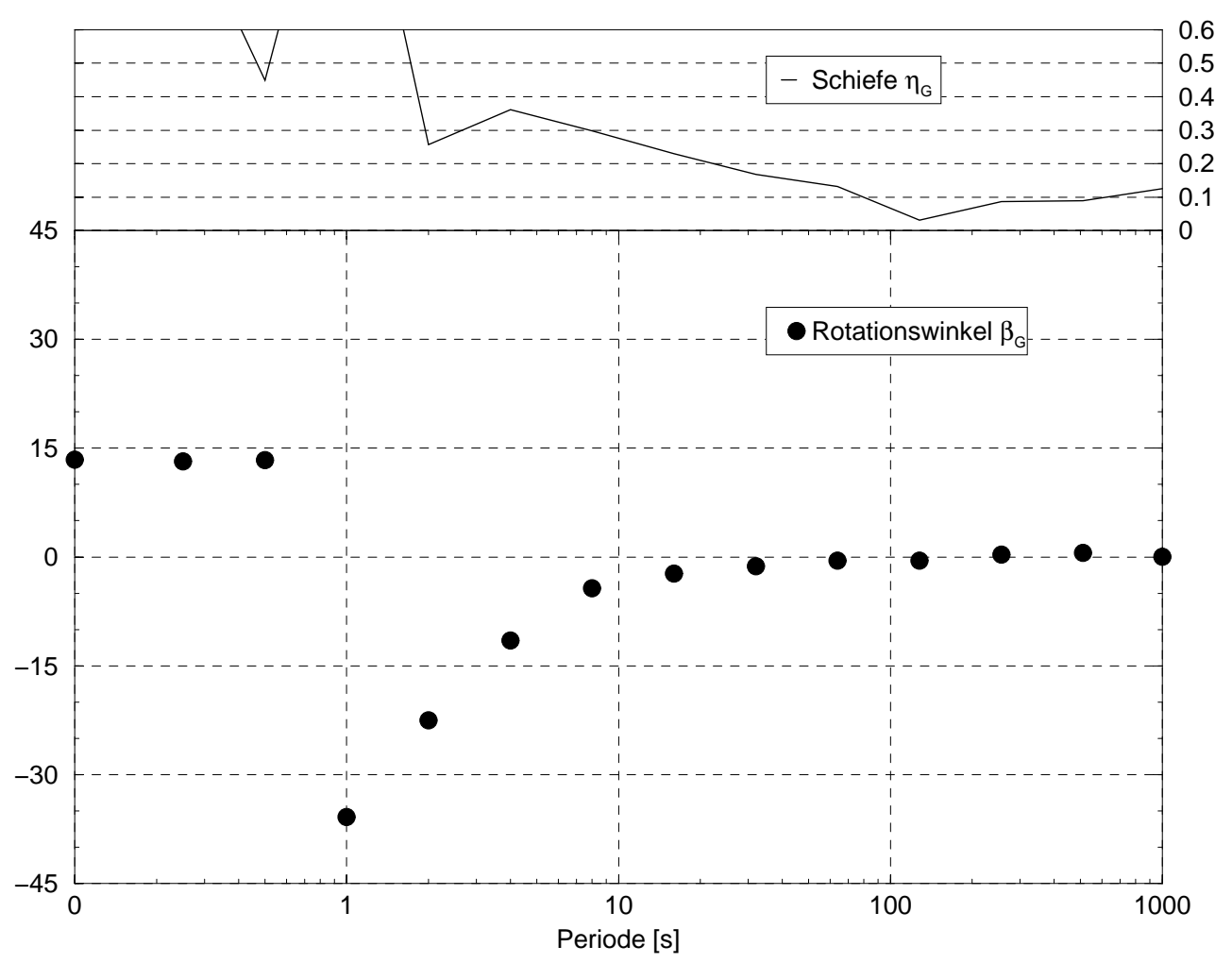

Abbildung 2.11: Frequenzgang des phasensensitiven Rotationswinkels $\beta_{G}$ und der Schiefe $\eta_{G}$ aus dem Perturbationstensor für das Modell N (Abbildung 2.10).

Man erkennt, daß die phasensensitive Schiefe bis etwa $10 \mathrm{~s}$ sehr groß ist. Das bedeutet, daß in diesem Bereich der Ansatz (2.32) nicht zutrifft. Die lokale Anomalie hat bei sehr kurzen Perioden hauptsächlich einen induktiven Effekt. Tatsächlich wird auch bei der Bestimmung des Richtungswinkels erst ab etwa 10 s die richtige Streichrichtunng von $0^{\circ}$ wiedergegeben, und zwar mit einer guten Stabilität und einer geringen Schwankungsbreite. 


\section{Die Feldmessungen}

\subsection{Zur Geologie des Rheinischen Schiefergebirges}

\subsection{1 Überblick über die geologische Entwicklung}

Mitteleuropa erhielt während der Phase der variszischen Orogenese ${ }^{1}$ vor 400 - 250 Millionen Jahren seine endgültige tektonisch-metamorphe Ausformung (WALTER, 1995). In seiner Gesamtheit erstreckt sich das variszische Gebirge als etwa 500 km breiter Faltengürtel vom französischen Zentralplateau durch West- und Mitteldeutschland bis zur Elbelinie, und von dort weiter in die Sudeten bis in das Polnische Mittelgebirge (MURAWSKI, 1992). Es wird in mehrere Zonen unterteilt, die in Abbildung 3.1 dargestellt sind. Sie unterscheiden sich hinsichtlich ihrer sedimentär-magmatischen und tektonisch-metamorphen Entwicklung.

Das Rheinische Schiefergebirge ist ein Teil der Rhenoherzynischen Zone des variszischen Gebirges. Es wird vorwiegend aus Sedimentgesteinen des Devons und des Unterkarbons aufgebaut, die während der variszischen Faltungsära im Oberkarbon zum größten Teil verfaltet und verschiefert wurden. Tiefengesteine sind im Rheinischen Schiefergebirge nicht aufgeschlossen.

Die einzelnen Strukturen weisen größtenteils eine südwest-nordöstliche Streichrichtung auf. Diese wird im folgenden als die variszische Richtung bezeichnet ${ }^{2}$.

\footnotetext{
Abgrenzung

Im Westen schließt an das Linksrheinische Schiefergebirge direkt das Paläozoikum der Ardennen an, im Nordwesten wird es durch das Tertiär und Quartär der Niederrheinischen Bucht überdeckt. Im Osten verschwindet das variszische Stockwerk des Schiefergebirges unter dem Buntsandstein und Tertiär der Hessischen Senke. Am Südrand taucht der Hunsrück unter das Perm der Saar-Nahe-Senke ab (Hunsrück-Südrand-Störung).

${ }^{1}$ Wesentlicher Bestandteil und Höhepunkt der variszischen Gebirgsbildung war der Zusammenschluß von Gondwana und Laurasia zum Superkontinent Pangaea im oberen Paläozoikum (z. B. PRESS \& SIEVER, 1995; IRVING, 1977). Im Detail ist die Geodynamik der variszischen Orogenese aber noch nicht verstanden.

2 Diese Bezeichnung ist üblich, allerdings besitzt das variszische Gebirge insgesamt kein einheitliches Streichen, so daß sie zum Teil abgelehnt wird (MURAWSKI, 1992). Eine anderer Ausdruck für diese Richtung ist erzgebirgisch nach dem variszischen Erzgebirge.
} 


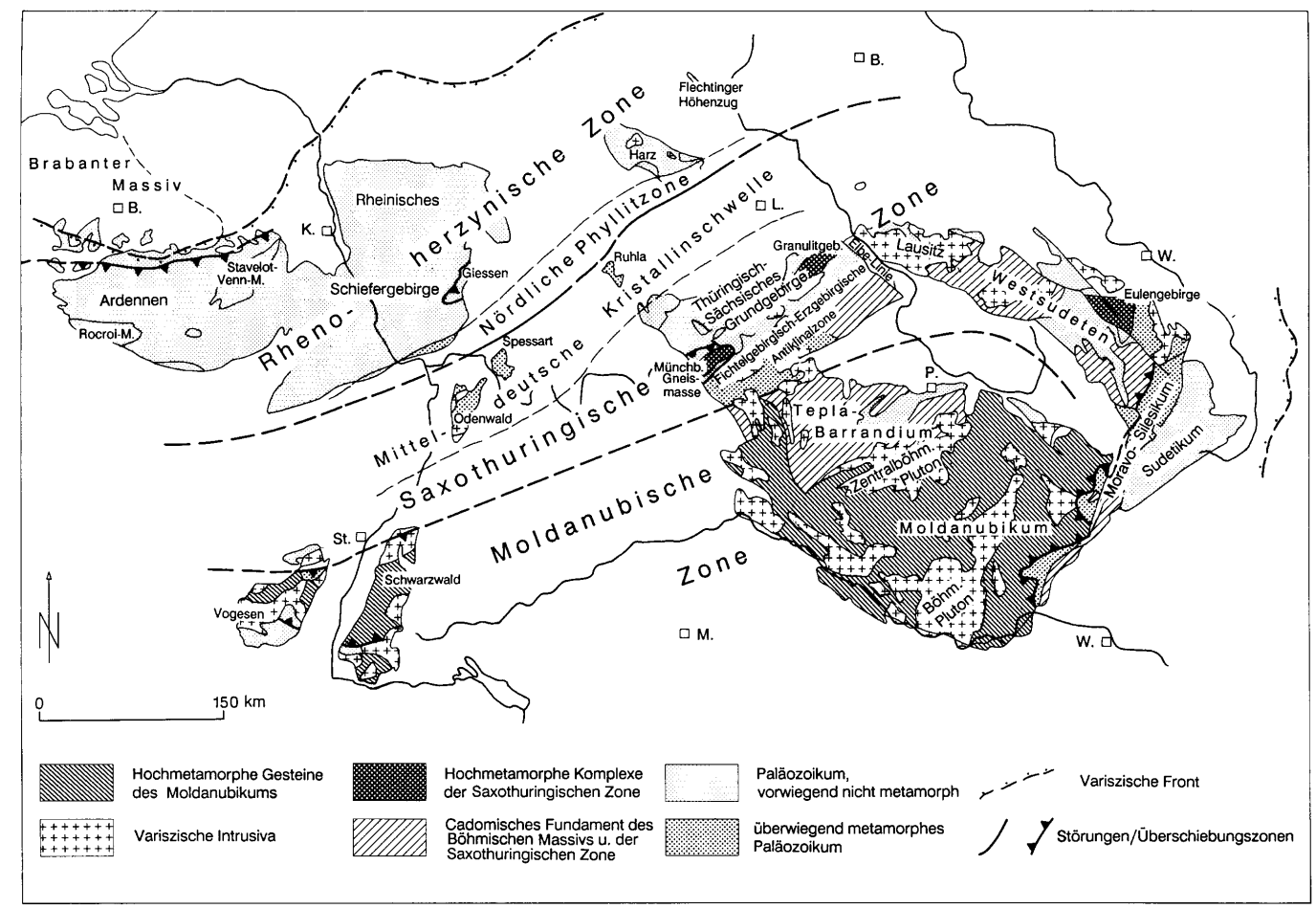

Abbildung 3.1: Das variszische Gebirge Mitteleuropas. Aus: (WALter, 1995).

\subsubsection{Die Hebung des Rheinischen Schildes}

Nach der Phase der variszischen Gebirgsbildung ist das Rheinische Schiefergebirge im wesentlichen Festland geblieben. Danach war das Faltengebirge verschiedenen Stadien der Verwitterung und Abtragung unterworfen, und es bildete sich der Gebirgsrumpf der Rheinischen Masse (MURAWSKI et al., 1983; MEYER et al., 1983).

Während der Oberkreide und des Alttertiär besaß dieses Gebiet noch ein recht flaches Relief. Der Aufstieg des Gebirgsrumpfes begann wahrscheinlich erst während des Eozäns vor etwa 38 - 55 Millionen Jahren (MEYER, 1994), wobei sich diese Hebung während der letzten 800.000 Jahre merklich beschleunigte. Dabei hob sich der Rheinische Schild ${ }^{3}$ nicht gleichmäßig als kompletter Block, sondern in mehreren Schollen mit unterschiedlichen Hebungsraten.

Das Ausmaß dieses Aufstiegs während der letzten 800.000 Jahre lag in der Regel bei mindestens $50 \mathrm{~m}$, Maximalwerte findet man in der Eifelregion (Kelberg) und im östlichen Hunsrück mit mehr als 200 m (MEYER \& STETS, 1998a, 1998b).

Die Hebungsphase dauert bis heute an, im nördlichen Teil der Eifel und im Venn-Sattel werden Hebungsraten bis zu 1.6 mm/Jahr gemessen (MÄLZER et al., 1983).

\footnotetext{
${ }^{3}$ Im folgenden wird der Begriff Rheinischer Schild für den jungen Hebungsbereich des Rheinischen Schiefergebirges verwendet.
} 


\subsubsection{Das europäische Riftsystem}

Das europäische Riftsystem hat eine Länge von etwa $1100 \mathrm{~km}$ und erstreckt sich quer über Zentraleuropa von der Nordsee bis zum Mittelmeerraum (PRODEHL et al., 1995).

Man versteht unter einem kontinentalen Rift eine langgestreckte, schmale Depression der Erdoberfläche, deren Absenkung in der Regel an parallelen tektonischen Brüchen erfolgt. Unter diesen Gebieten läßt sich eine Extension der Kruste bzw. Ausdünnung der Lithosphäre nachweisen. Das europäische Riftsystem gehört zu der Gruppe der modernen Rifts. Diese zeichnen sich durch eine diffuse seismische Tätigkeit, relative Absenkung und Hebung von Krustenblöcken und einen bis in die jüngste geologische Vergangenheit anhaltenden Vulkanismus aus (EISBACHER, 1996). In Abbildung 3.2 ist der grobe Aufbau des Riftsystems dargestellt.

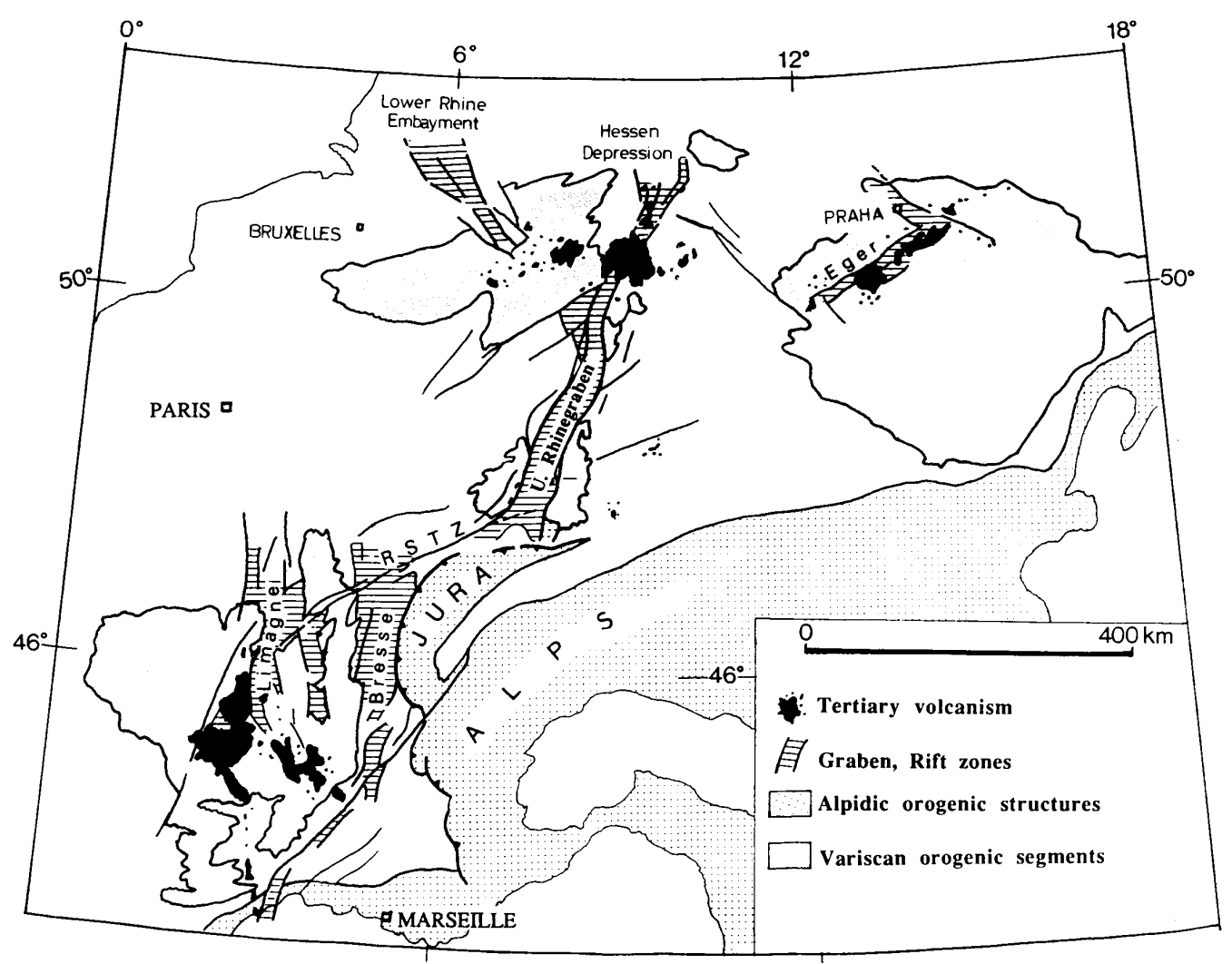

Abbildung 3.2: Das europäische Riftsystem. Aus: (ProdeHL ET AL., 1995)

Der Rheingraben zwischen Basel und Frankfurt bildet den zentralen Teil. Er spaltet am nördlichen Ende in zwei Äste auf. Richtung Nordosten folgt sein Verlauf der Hessischen Senke und dem Leinegraben und verschwindet dann unter den jungen Sedimenten der Norddeutschen Tiefebene. Der andere Zweig verläuft Richtung Nordwesten durch das Rheinische Massiv und tritt auf der anderen Seite in der Niederrheinischen Bucht hervor. Man erkennt in Abbildung 3.2, daß der Vulkanismus zum Teil dem Verlauf der Graben- 
strukturen folgt, wie etwa im Erzgebirge. Die Vulkankette, die sich von der Rhön bis zur Eifel erstreckt, korreliert jedoch nicht mit einer Grabenstruktur. Auffällig ist weiterhin, daß im Rheingraben vulkanische Aktivität fast vollständig fehlt.

\subsubsection{Quartärer Vulkanismus in der Eifel}

Im Tertiär und Quartär war das Rheinische Schiefergebirge Schauplatz eines ausgeprägten Vulkanismus. Es werden mehrere Vulkanfelder unterschieden (Abbildung 3.3). Im Siebengebirge und im Westerwald konzentrierte sich die vulkanische Tätigkeit im wesentlichen auf den Zeitraum oberes Oligozän bis unteres Miozän vor etwa 20 - 30 Millionen Jahren sowie auf den Übergang Miozän/Pliozän vor rund 5 - 10 Millionen Jahren.

Das Gebiet der Eifel wird nochmals unterteilt in die Westeifel, Hocheifel und die Osteifel. Während tertiäre Vulkanite vor allem in der Hocheifel verbreitet sind, findet man in der Westeifel und der Osteifel hauptsächlich vulkanisches Gestein des Quartär.

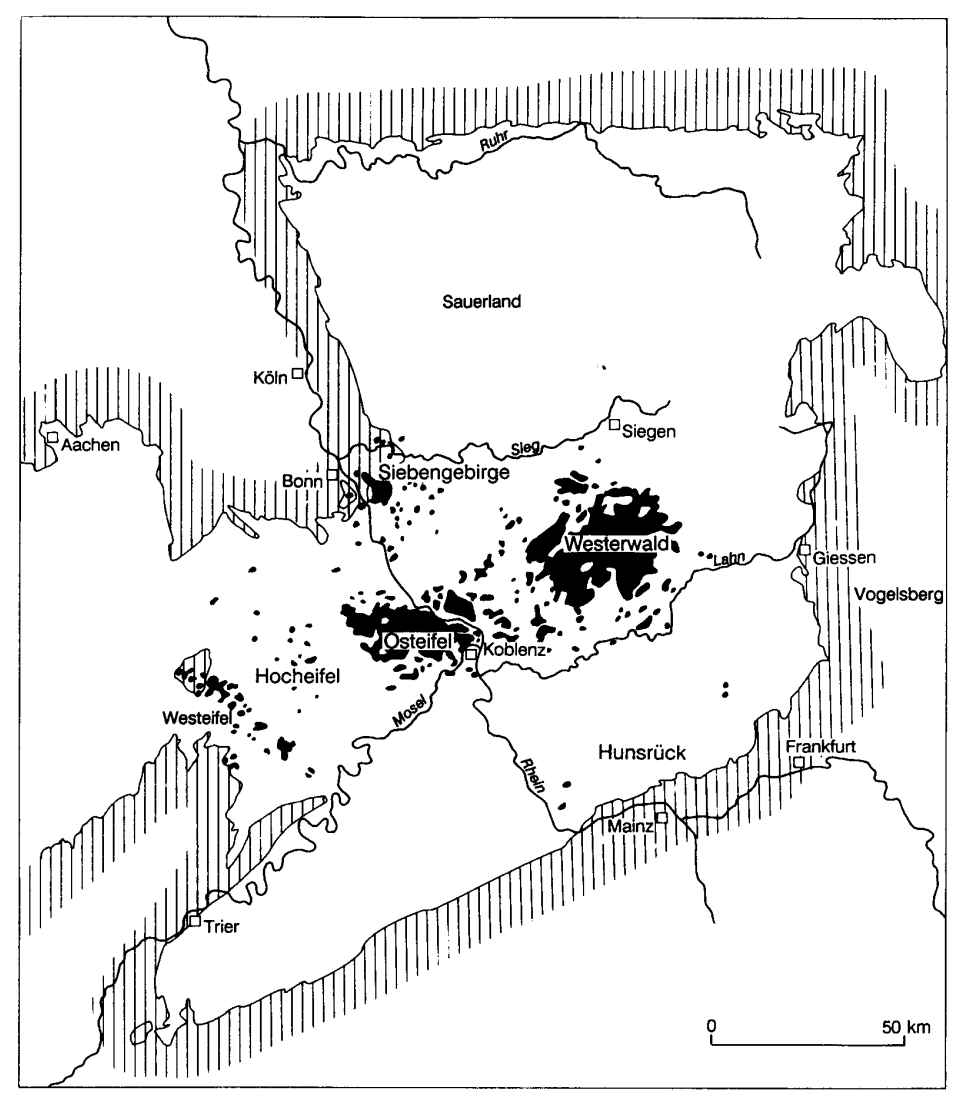

Abbildung 3.3: Die Vulkanfelder des Rheinischen Schiefergebirges. Aus: (WALTER, 1995). 
Das westliche Feld bedeckt eine Fläche von rund $600 \mathrm{~km}^{2}$. Es hat eine Länge von etwa $50 \mathrm{~km}$ und erstreckt sich in nordwest-südöstlicher Richtung. Insgesamt sind 250 Vulkane vorhanden, das eruptierte Magmavolumen wird auf etwa $5 \mathrm{~km}^{3}$ geschätzt.

Das östliche Gebiet in der Umgebung des Laacher Sees ist mit $400 \mathrm{~km}^{2}$ Fläche und etwa 100 Vulkanzentren etwas kleiner. Es besitzt eine längliche Form mit einer Ausdehnung von rund $35 \mathrm{~km}$ in nordwest-südöstlicher Richtung (SCHMINCKE et al., 1983). Das insgesamt eruptierte Magmenvolumen liegt mit etwa $15 \mathrm{~km}^{3}$ deutlich über dem der Westeifel. Maßgeblichen Anteil daran hatte die Eruption, durch die vor etwa 11.000 - 13.000 Jahren der Laacher See entstand. Das Volumen des ausgeworfenen Materials (etwa $5 \mathrm{~km}^{3}$ Bimstuffe) war mit dem des Vesuv (79, Italien) vergleichbar und überstieg dasjenige des Mt. St. Helens (1980, Washington) um etwa das 10-fache (SCHMincKe, 1986). Die Asche wurde über weite Teile Deutschlands und der angrenzenden Länder verteilt. Ablagerungen wurden u. a. auf Bornholm und im Gebiet zwischen Bodensee und Genfer See gefunden (WALTER, 1995). Die dabei entstandene dünne Bimslage dient zum Teil als wichtiger Leithorizont für die darunter und darüber liegenden Schichten des Quartärs.

Die charakteristischten Vulkanformen der Eifel, die Maare, findet man überwiegend in der Westeifel. Sie entstanden in Tälern, in denen in Spalten aufsteigendes Magma mit Grundwasser in Kontakt kam. Dabei wurden phreatomagmatische Explosionen verursacht, die das Nebengestein fragmentierten und zum Teil herausschleuderten. Dadurch entstand eine instabile Eruptionskammer, die schließlich einbrach. Die kraterartige Senke bezeichnet man als Maar, in dem sich meist ein See oder Moor bildete.

Das jüngste Maar, das Ulmener Maar, ist mit einem Alter von möglicherweise weniger als 10.000 Jahren (MEYER, 1994) noch jünger als der Laacher See und somit die jüngste vulkanische Bildung in Mitteleuropa.

Ob allerdings die vulkanische Tätigkeit in der Eifel insgesamt beendet ist, kann nicht mit Sicherheit gesagt werden. 


\subsection{Die Meßkampagne}

\subsubsection{Zur Auswahl der Stationsstandorte}

Bei der Auswahl einer geeigneten Lokation für eine Station zur Aufzeichnung langperiodischer elektromagnetischer Felder muß grundsätzlich darauf geachtet werden, daß anthropogene Störungen vermieden werden. Aus diesem Grund wurden die Stationen in der Regel in abgelegenen Waldgebieten aufgebaut. Auch mußte darauf geachtet werden, daß keine Bahnstrecken, Sendemasten, unterirdisch verlegte Gasleitungen oder ähnliches in der Nähe waren. Selbst ein elektrischer Weidezaun kann das elektrische Feld so stark stören, daß die aufgezeichneten Daten unbrauchbar sind. Aus diesem Grund wurden die endgültigen Standorte in Absprache mit den zuständigen Forstbeamten ausgewählt.

Die großräumige Planung des Stationsnetzes geschah unter Berücksichtigung mehrerer Aspekte:

- Die quartären Vulkangebiete der Westeifel und der Osteifel sollten im Zentrum des Arrays liegen.

- Die Größe des Arrays wurde so gewählt, daß eine Auflösung des obereren Mantels möglich ist, dabei sollte die Bedeckung möglichst gleichmäßig sein.

- Die nördliche Begrenzung war durch die Industriegebiete im Raum Köln und das Ruhrgebiet gegeben, im Süden durch das Ballungsgebiet um Frankfurt.

- Die Ausrichtung des Meßnetzes wurde am variszischen Streichen orientiert. Mögliche Profile für spätere zweidimensionale Analysen, etwa Inversionsrechnungen, liegen somit parallel und senkrecht zu den Faltenstrukturen. Zudem wurde das Gitter so entworfen, daß bereits früher vermessene Profile (BANTIN 1997; SCHNEIDER, 1998) direkt angeschlossen werden können.

Die Standorte der Stationen sind in Abbildung 3.4 dargestellt. Insgesamt überdeckte das Array eine Fläche von etwa $140 \mathrm{~km} \times 130 \mathrm{~km}$, der durchschnittliche Stationsabstand betrug 30 - $40 \mathrm{~km}$. Die Verdichtung des Stationsnetzes im Nordwesten wurde im Rahmen einer zweiten Kampagne realisiert (vgl. Kapitel 3.2.3), nachdem bereits erste Ergebnisse vorlagen und es deutliche Hinweise auf eine Anomalie im magnetischen Feld mit Zentrum unter Hillesheim (Station HIL) gab (vgl. dazu Kap. 4). 


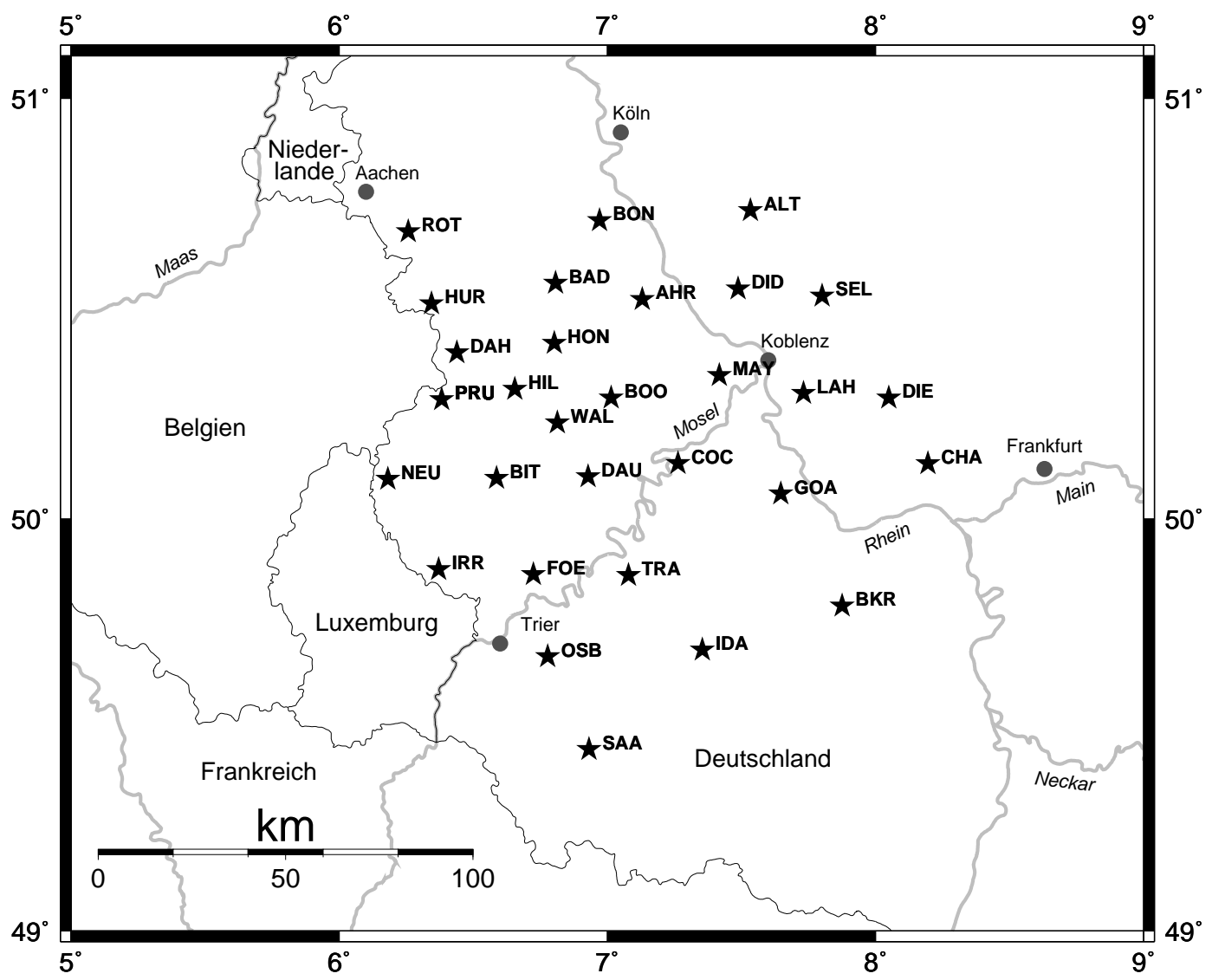

Abbildung 3.4: Die Standorte der Meßstationen im Rheinischen Schiefergebirge.

\subsubsection{Die Meßapparatur}

- Messung des elektrischen Feldes

Das elektrische Feld $E$ wird als Spannungsabfall $U$ zwischen zwei Elektroden im Abstand $d$ gemessen:

$$
E=\frac{U}{d}
$$

Dabei wird angenommen, daß sich das elektrische Feld über die Elektrodendistanz nicht ändert. Üblicherweise liegt $d$ in einer Größenordnung von $50 \mathrm{~m}-100 \mathrm{~m}$. Insgesamt wurden jeweils vier, etwa $30-50 \mathrm{~cm}$ tief eingegrabene Elektroden verwendet, die das elektrische Feld in magnetischer Nord-Südrichtung und West-Ostrichtung gemessen haben.

Um elektrochemische Effekte zu vermeiden, wurden unpolarisierbare Elektroden verwendet. Diese bestehen aus einem Metallstab, der in eine gesättigte Lösung seines eigenen Salzes eintaucht und in einem porösen Rohr untergebracht ist. Es wurden Elektroden vom Typ Ag-AgCl verwendet (FILlOuX, 1987). Sie befinden sich ihrerseits in einem PVC-Rohr, welches mit gesättigter KCl-Lösung gefüllt ist. Der 
Kontakt zum Erdreich wird über Keramikfritten hergestellt (JUNGE, 1990). Diese Elektroden zeichnen sich durch eine sehr gute Langzeitstabilität aus.

- Messung des magnetischen Feldes

Das magnetische Feld wurde mit Fluxgate-Magnetometern gemessen. Die eigentliche Aufzeichnungseinheit ist dabei in einem korrosionsfesten Kunstoffgehäuse (Göttinger Bauart) untergebracht und wurde ebenfalls etwa $50 \mathrm{~cm}$ tief vergraben, nachdem es im Erdmagnetfeld ausgerichtet und mit Hilfe einer Libelle waagerecht justiert worden war.

- Die Aufzeichungsapparatur

Sämtliche Daten wurden mit dem von Dr. E. Steveling am Institut für Geophysik in Göttingen entwickelten RAP-System (RAM-Apparatur) aufgezeichnet (STEVELING \& LEVEN, 1992).

Der Datenlogger ist für die Aufzeichnung niederfrequenter Signale unter Geländebedingungen konzipiert worden. Er besitzt 8 analoge Eingangskanäle $( \pm 5 \mathrm{~V})$ von denen jeder über einen eigenen 16 bit AD-Wandler verfügt, so daß die eingehenden Signale separat digitalisiert werden können. Die Abtastrate kann zwischen 1/64 s und $60 \mathrm{~s}$ eingestellt werden. Sämtliche aufgezeichneten Daten werden auf statischen Halbleiterspeichern mit einer Kapazität von 8 Mb abgelegt.

Um an verschiedenen Orten simultan mit mehreren Datenloggern messen zu können, ist es möglich, die interne Uhr des Loggers (Genauigkeit 1 ppm) über eine externe DCF- oder GPS-Uhr zu synchronisieren.

Die Stromversorgung kann über herkömmliche $12 \mathrm{~V}$ Autobatterien erfolgen. Die übliche Bestückung mit zwei Akkus (jeweils etwa $80 \mathrm{Ah}$ ) gestattet bei einer Abtastrate von $2 \mathrm{~s}$ unter normalen Witterungsbedingungen eine ununterbrochene Laufzeit im Gelände von etwa 2 Wochen. Bei einer hohen Abtastrate wird die maximal mögliche Laufzeit allerdings durch den verfügbaren Speicherplatz begrenzt. Sämtliche Geräte werden in einer verschließbaren Aluminiumbox untergebracht.

\subsubsection{Durchführung der Feldmessungen}

Die gesamte Meßkampagne in der Eifel gliederte sich in eine Hauptkampagne im Sommer 1997 (23 Stationen) und in eine kleinere Kampagne im Winter 1998 (8 Stationen).

Zur Durchführung waren neben den Geräten des Göttinger Instituts für Geophysik weitere Geräte erforderlich, die von der Freien Universität Berlin und dem GeoForschungsZentrum Potsdam zur Verfügung gestellt wurden ${ }^{4}$. Sämtliche Geräte waren vom Typ identisch und unterschieden sich nur in Details, so etwa bei den verwendeten Filtern oder dem mechanischen Aufbau der Elektroden und der Magnetometer.

\footnotetext{
${ }^{4}$ An einigen Stationen wurden außerdem in Zusammenarbeit mit der FU Frankfurt AMT-Messungen durchgeführt.
} 
Die Standorte der einzelnen Stationen sind bereits in Abbildung 3.4 dargestellt worden. In Tabelle 3.1 sind die Standzeiten der einzelnen Stationen aufgelistet. An einigen Stationen führten technische Probleme dazu, daß die eigentliche Registrierdauer einen deutlich kürzeren Zeitraum umfaßt, als die Standzeit insgesamt. An der Station HIL wurden zu Vergleichszwecken im Rahmen beider Kampagnen Messungen durchgeführt.

Es ist außerdem gekennzeichnet, ob die gewonnenen Daten zur Auswertung nach der Methode der Magnetotellurik oder der erdmagnetischen Tiefensondierung verwendet wurden. In der Regel ist das elektrische Signal weit stärker gestört als das magnetische. An einigen Stationen war dessen Qualität für eine sinnvolle Auswertung nicht ausreichend, so daß dann nur die Aufzeichnung des magnetischen Feldes verwendet werden konnte. In einem Fall erwies sich die Datenqualität aller Feldkomponenten als so schlecht, daß diese Station (BON) für die Auswertung insgesamt gestrichen wurde. 


\begin{tabular}{|c|c|c|c|c|c|}
\hline Station & Standzeit & Registrierdauer & Registrierapparatur & MT & GDS \\
\hline AHR & $23.7 .97-26.8 .97$ & 35 Tage & Berlin & - & - \\
\hline ALT & $21.7 .97-1.9 .97$ & 43 Tage & Berlin & - & $\bullet$ \\
\hline BAD & $14.10 .98-10.12 .98$ & 44 Tage & Göttingen & $\bullet$ & $\bullet$ \\
\hline BIT & $5.8 .97-2.9 .97$ & 29 Tage & Potsdam & & $\bullet$ \\
\hline BKR & $6.8 .97-2.9 .97$ & 28 Tage & Potsdam & $\bullet$ & $\bullet$ \\
\hline BON & $16.10 .98-10.12 .98$ & 44 Tage & Göttingen & & \\
\hline $\mathrm{BOO}$ & $2.8 .97-4.9 .97$ & 34 Tage & Göttingen & $\bullet$ & $\bullet$ \\
\hline CHA & $5.8 .97-2.9 .97$ & 29 Tage & Potsdam & & $\bullet$ \\
\hline $\mathrm{COC}$ & $2.8 .97-3.9 .97$ & 23 Tage & Göttingen & $\bullet$ & $\bullet$ \\
\hline DAH & $15.10 .98-11.12 .98$ & 44 Tage & Göttingen & $\bullet$ & $\bullet$ \\
\hline DAU & $21.7 .97-3.9 .97$ & 45 Tage & Göttingen & $\bullet$ & $\bullet$ \\
\hline DID & $5.8 .97-1.9 .97$ & 28 Tage & Potsdam & & $\bullet$ \\
\hline DIE & $23.7 .97-2.9 .97$ & 42 Tage & Berlin & $\bullet$ & $\bullet$ \\
\hline FOE & $6.8 .97-2.9 .97$ & 28 Tage & Potsdam & $\bullet$ & $\bullet$ \\
\hline GOA & $2.8 .97-3.9 .97$ & 33 Tage & Göttingen & $\bullet$ & $\bullet$ \\
\hline HIL & $22.10 .98-10.12 .98$ & 43 Tage & Göttingen & $\bullet$ & $\bullet$ \\
\hline HON & $15.10 .98-11.12 .98$ & 44 Tage & Göttingen & $\bullet$ & $\bullet$ \\
\hline HUR & $7.8 .97-2.9 .97$ & 27 Tage & Potsdam & $\bullet$ & $\bullet$ \\
\hline IDA & $13.8 .97-2.9 .97$ & 21 Tage & Potsdam & & $\bullet$ \\
\hline IRR & $22.7 .97-3.9 .97$ & 44 Tage & Göttingen & & $\bullet$ \\
\hline LAH & $6.8 .97-1.9 .97$ & 20 Tage & Potsdam & $\bullet$ & $\bullet$ \\
\hline MAY & $7.8 .97-4.9 .97$ & 22 Tage & Göttingen & $\bullet$ & $\bullet$ \\
\hline NEU & $21.7 .97-3.9 .97$ & 45 Tage & Göttingen & & $\bullet$ \\
\hline OSB & $13.8 .97-2.9 .97$ & 21 Tage & Potsdam & $\bullet$ & $\bullet$ \\
\hline PRU & $22.10 .98-10.12 .98$ & 43 Tage & Göttingen & $\bullet$ & $\bullet$ \\
\hline ROT & $15.10 .98-9.12 .98$ & 41 Tage & Göttingen & & $\bullet$ \\
\hline SAA & $6.8 .97-2.9 .97$ & 24 Tage & Potsdam & & $\bullet$ \\
\hline SEL & $21.7 .97-1.9 .97$ & 43 Tage & Berlin & $\bullet$ & $\bullet$ \\
\hline TRA & $4.8 .97-3.9 .97$ & 31 Tage & Göttingen & $\bullet$ & $\bullet$ \\
\hline WAL & $22.10 .98-11.12 .98$ & 30 Tage & Göttingen & $\bullet$ & $\bullet$ \\
\hline
\end{tabular}

Tabelle 3.1: Übersicht über die Registrierzeiten der einzelnen Meßstationen. Es ist gekennzeichnet, ob die an der jeweiligen Station gewonnenen Daten zur Auswertung nach der Methode der Magnetotellurik und/oder der erdmagnetischen Tiefensondierung verwendet wurden. 


\subsubsection{Aufzeichnung elektromagnetischer Zeitreihen}

In der Regel werden an den Feldstationen alle Komponenten des magnetischen Feldes sowie die horizontalen Komponenten des elektrischen Feldes aufgezeichnet.

Die Taktrate beträgt durchgehend $2 \mathrm{~s}$. Das Datenmaterial liegt somit in Form von diskreten Zeitreihen $x=\left\{x\left(t_{i}\right), \ldots, x\left(t_{M}\right)\right\}, y=\left\{y\left(t_{i}\right) \ldots y\left(t_{M}\right),\right\}, z=\ldots$ mit dem Zeitabstand $\Delta t=t_{i+1}-t_{i}=2 \mathrm{~s}$ vor.

Die Länge dieser Zeitreihen ist unterschiedlich und hängt davon ab, wie oft die Stationen gewartet wurden, bzw. ob unvorhergesehene Störungen auftraten. Hier war der Ablauf der Feldmessungen so organisiert, daß alle Stationen regelmäßig im Abstand von etwa einer Woche kontrolliert und gewartet wurden, die Dauer der einzelnen Zeitabschnitte lag in der Regel also maximal bei etwa einer Woche.

In Abbildung 3.5 ist ein Registrierbeispiel der Station SEL für alle Feldkomponenten über einen Zeitraum von etwa 20 min dargestellt.
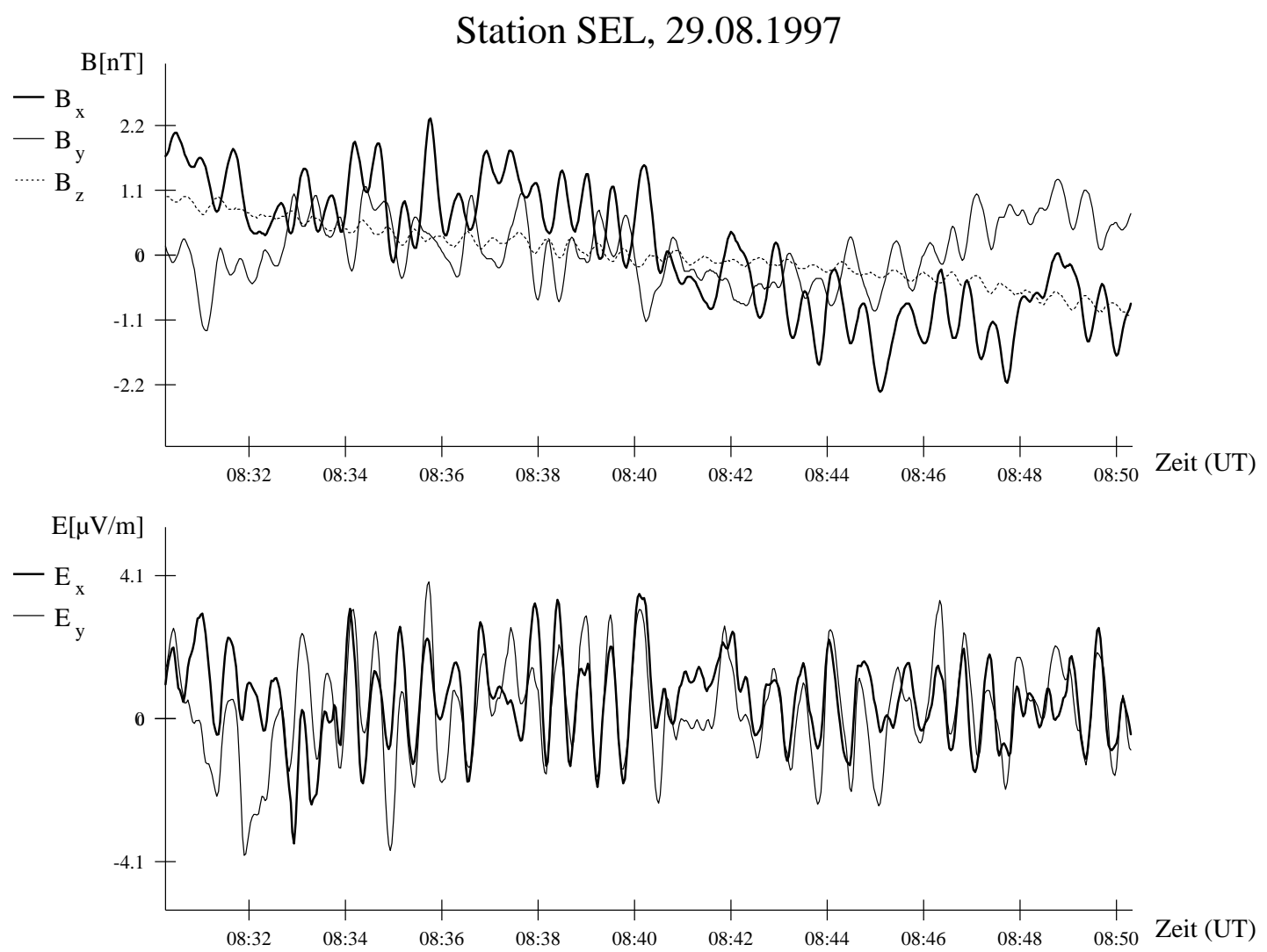

Abbildung 3.5: Registrierbeispiel der Station SEL für eine Abtastrate $\Delta t=2 \mathrm{~s}$.

Man erkennt gut die Korrelation der einzelnen Feldkomponenten. Die Amplitude der magnetischen Feldvariationen ist in diesem Beispiel mit maximal etwa 3 nT recht gering. An magnetisch unruhigen Tagen sind Amplituden von 50 nT - 100 nT möglich (etwa bei Bay-Störungen, vergl. Kap. 1.1). Erfahrungsgemäß sind die Schwankungen in der verti- 
kalen Magnetfeldkomponente viel geringer, dies wird auch in dem dargestellten Beispiel deutlich. Die Amplitude von $B_{z}$ liegt unter $1 \mathrm{nT}$.

Anthropogene Störungen der elektromagnetischen Signale machen sich in erster Linie durch vereinzelt oder periodisch auftretende Peaks in der Zeitreihe bemerkbar. Zum Teil ist den Daten auch ein kurzperiodisches Rauschen überlagert. In obigem Beispiel sind sowohl im elektrischen als auch im magnetischen Feld keine Störungen zu erkennen.

\subsection{Datenanalyse}

Im folgenden wird das Prozessing, also die Verarbeitung der aufgezeichneten Zeitreihen zu den frequenzabhängigen Übertragungsfunktionen in knapper Form dargestellt. Allgemeine Darstellungen der Spektralanalyse findet man beispielsweise bei JENKINS \& WATTS (1968) oder ButTKus (1991). Spezielle Aspekte der hier angewandten Methoden werden ausführlich bei JANTOS (1998) oder BAHR \& BRUTON (1994) behandelt.

\subsubsection{Vorbereitung der Rohdaten}

Sämtliche Rohdaten werden gesichtet und einer ersten visuellen Bewertung unterzogen. Daten, die offensichtlich stark gestört sind, werden so bereits vor der eigentlichen Analyse ausgesondert und gehen nicht in die Auswertung mit ein. Auch Zeitabschnitte, die keine oder nur geringe Anregung aufweisen, sind nicht zur Auswertung geeignet.

Darüberhinaus wird darauf geachtet, daß während der Registrierdauer keine starken magnetischen Stürme auftreten. In diesem Fall wäre es möglich, daß die quasi-homogene Näherung nicht mehr erfüllt ist. Das heißt, daß sich Quelleninhomogenitäten direkt in den aufgezeichneten Übertragungsfunktionen abbilden. Die Auswahl entsprechend ungeeigneter Zeitabschnitte wird anhand von Kp-Indizes vorgenommen.

Die dann endgültig zur Analyse vorgesehenen Daten liegen nach dieser Vorbehandlung in Form von Zeitfenstern mit einer bestimmten Länge $N \cdot \Delta t$ vor. Diese kann prinzipiell frei gewählt werden und hängt von dem Periodenbereich ab, der ausgewertet werden soll ${ }^{5}$.

Um die Randwerte der einzelnen Zeitfenster abzusenken, werden die Daten mit der Hanning-Fensterfunktion (Gleichung A.1) multipliziert. Ein etwaiger linearer Trend in den Daten wird entfernt.

Nach einer Fouriertransfomation erhält man aus diesen Zeitreihen ein erstes Rohspektrum mit den Fourierkoeffizienten $X=\left\{X_{j}\right\}, Y=\left\{Y_{j}\right\}, \ldots$ an den Fourierfrequenzen $f_{s}$.

Vor der weiteren Analyse müssen diese kalibriert werden. Dazu werden sie mit einem geräteabhängigen Skalenfaktor multipliziert, um Werte in SI-Einheiten zu erhalten $(B$ in nT und $E$ in $\mu \mathrm{V} / \mathrm{km}$ ). Außerdem wird der Einfluß des während der Aufzeichnung verwendeten Tiefpaßfilters entfernt. Dazu werden die Fourierkoeffizienten im Frequenzbereich

\footnotetext{
${ }^{5}$ Bei der anschließenden diskreten Fouriertransformation wird die Methode der FFT (Fast FourierTransformation) verwendet. Aus diesem Grund sollte die Länge des Zeitfensters so gewählt werden, daß $N=2^{n}$ ist ( $n$ ganze positive Zahl $\leq 10$ ).
} 
durch die entsprechenden Übertragungsfunktionen des jeweiligen Filters dividiert.

\subsubsection{Berechnung von geglätteten Spektren}

Aus den komplexen Fourierkoeffizienten $X, Y$ können reelle Autospektren

$$
S_{X X}=X X^{*}
$$

und komplexe Kreuzspektren

$$
S_{X Y}=X Y^{*}
$$

gebildet werden $^{6}$. Da die Fourierfrequenzen der FFT von der jeweiligen Länge des Zeitfensters und der Abtastrate abhängig sind, werden im Vorfeld bestimmte Auswertefrequenzen $f_{e}$ festgelegt.

Diese Frequenzen werden so gewählt, daß sie bei einer Auftragung im logarithmischen Maßstab gleiche Abstände aufweisen und 6 oder 7 Werte in eine Dekade fallen. In Tabelle 3.2 sind die in dieser Arbeit verwendeten Frequenzen aufgelistet.

\begin{tabular}{|c|c||c|c|}
\hline$f_{e}[\mathrm{~Hz}]$ & $T_{e}[\mathrm{~s}]$ & $f_{e}[\mathrm{~Hz}]$ & $T_{e}$ \\
\hline \hline $2.44 \cdot 10^{-4}$ & 4096 & $5.85 \cdot 10^{-3}$ & 171 \\
\hline $3.66 \cdot 10^{-4}$ & 2731 & $7.81 \cdot 10^{-3}$ & 128 \\
\hline $4.88 \cdot 10^{-4}$ & 2048 & $1.18 \cdot 10^{-2}$ & 85 \\
\hline $7.33 \cdot 10^{-4}$ & 1365 & $1.56 \cdot 10^{-2}$ & 64 \\
\hline $9.77 \cdot 10^{-4}$ & 1024 & $2.32 \cdot 10^{-2}$ & 43 \\
\hline $1.46 \cdot 10^{-3}$ & 683 & $3.13 \cdot 10^{-2}$ & 32 \\
\hline $1.95 \cdot 10^{-3}$ & 512 & $4.76 \cdot 10^{-2}$ & 21 \\
\hline $2.93 \cdot 10^{-3}$ & 341 & $6.25 \cdot 10^{-2}$ & 16 \\
\hline $3.91 \cdot 10^{-3}$ & 256 & $9.09 \cdot 10^{-2}$ & 11 \\
\hline
\end{tabular}

Tabelle 3.2: Die in dieser Arbeit verwendeten Auswertefrequenzen.

Um die statistische Sicherheit zu erhöhen, werden an diesen Auswertefrequenzen geglättete Spektren $\left\langle X X^{*}\right\rangle$ und $\left\langle X Y^{*}\right\rangle$ berechnet.

Dazu wird im Frequenzbereich über jeweils $N_{p}$ (Gleichung A.3) der Auswertefrequenz $f_{e}$ benachbarte Rohspektralwerte $S$ (die Indizes werden im folgenden fortgelassen) an den Fourierfrequenzen $f_{s(m-k)} \ldots f_{s(m+k)}$ ein gewichtetes Mittel berechnet:

$$
\bar{S}\left(f_{e}\right)=P_{0} S\left(f_{s(m)}\right)+\sum_{k=1}^{N_{p}-1} P_{k}\left[S\left(f_{s(m+k)}\right)+S\left(f_{s(m-k)}\right)\right] .
$$

\footnotetext{
${ }^{6}$ Der Stern * bezeichnet die konjugiert komplexen Werte.
} 
Als Gewichtsfunktion wird hier ein Parzen-Fenster $P$ verwendet (Gleichung A.2). Die so erhaltenen geglätteten Spektren der einzelnen Zeitfenster werden anschließend einer Bewichtung unterzogen und gestapelt. Als Gütekriterium dient dabei die bivariate quadratische Kohärenz (Gleichung A.4).

\subsubsection{Schätzung der Übertragungsfunktionen}

Sowohl in der Magnetotellurik als auch in der erdmagnetischen Tiefensondierung kann der Zusammenhang zwischen den Komponenten des elektrischen und magnetischen Feldes bzw. zwischen den Komponenten des anomalen und normalen Magnetfeldes durch ein lineares, bivariates System beschrieben werden.

Aus den Definitionen (2.2) und (1.20) geht bereits hervor, daß zur Bestimmung der einzelnen Elemente von $\underline{\mathbf{W}}$ und $\underline{\mathbf{Z}}$ mindestens zwei voneinander unabhängige Messungen der Feldkomponenten notwendig sind. Zur Berechnung der Übertragungsfunktionen kann dann die Methode der kleinsten Quadrate verwendet werden.

Dazu wird angenommen, daß nur das elektrische Feld bzw. nur das anomale magnetische Feld fehlerbehaftet ist.

Allgemein kann der Zusammenhang folgendermaßen beschrieben werden:

$$
Z=C_{1} X+C_{2} Y+\Delta Z
$$

Entsprechend den Gleichungen (2.2) und (1.20) steht dabei $Z$ für $E_{x}, E_{y}$ bzw. $B_{x}^{a}, B_{y}^{a}, B_{z}^{a}, . X$ und $Y$ repräsentieren jeweils die Horizontalkomponenten des magnetischen Feldes (lokal bzw. an der Referenzstation).

Der Fehler $\Delta Z$ soll minimiert werden. Liegen $N$ Messungen vor, ergeben sich aus der Bedingung

$$
\sum_{i=1}^{N}\left[\left(Z_{i}-C_{1} X_{i}-C_{2} Y_{i}\right)\left(Z_{i}-C_{1} X_{i}-C_{2} Y_{i}\right)^{*}\right]=\min !
$$

folgende Bestimmungsgleichungen für die Schätzwerte der Übertragungsfunktionen:

$$
\begin{aligned}
& \hat{S}_{Z X}=C_{1} \hat{S}_{X X}+C_{2} \hat{S}_{Y X} \\
& \hat{S}_{Z Y}=C_{1} \hat{S}_{X Y}+C_{2} \hat{S}_{Y Y} .
\end{aligned}
$$

Dabei bezeichnet $\hat{S}$ jeweils die nach Gleichung (3.2) geglätteten und über mehrere Zeitfenster gemittelten Spektren (Anhang A.3.3). Für die gesuchten Übertragungsfunktionen erhält man:

$$
\begin{aligned}
C_{1} & =\frac{\hat{S}_{Z X} \hat{S}_{Y Y}-\hat{S}_{Z Y} \hat{S}_{Y X}}{\hat{S}_{X X} \hat{S}_{Y Y}-\left|\hat{S}_{X Y}\right|^{2}} \\
C_{2}= & \frac{\hat{S}_{Z Y} \hat{S}_{X X}-\hat{S}_{Z X} \hat{S}_{X Y}}{\hat{S}_{X X} \hat{S}_{Y Y}-\left|\hat{S}_{X Y}\right|^{2}} .
\end{aligned}
$$


Für die Berechnung der Übertragungsfunktionen benötigt man also jeweils die Auto- und Kreuzspektren der beteiligten Feldkomponenten. Explizite Formeln für alle Elemente von $\underline{\mathbf{Z}}$ und $\underline{\mathbf{W}}$ finden sich im Anhang A.3.2. Dort sind ebenfalls Bemerkungen zur Bestimmung der Fehler aufgeführt. 


\section{Ergebnisse der Datenanalyse}

Im folgenden Kapitel werden die Ergebnisse der Datenanalyse zusammengefasst und die wesentlichen Aspekte herausgearbeitet. Dabei macht es die Fülle des Datenmaterials unumgänglich, auf die Daten einer einzelnen Station nur in Ausnahmefällen explizit einzugehen. Zum Teil werden einige Stationen herausgegriffen, die dann als Beispiel für das Verhalten der Meßgrößen in einer bestimmten Region oder im gesamten Meßgebiet gelten.

\section{1 Überblick}

In der Regel kann mit elektromagnetischen Methoden bei genügend großer Meßpunktdichte eine sehr gute laterale Auflösung hinsichtlich der Leitfähigkeitsverteilung erreicht werden.

Die vertikale Variation der Leitfähigkeit spiegelt sich im Frequenzverhalten der Übertragungsfunktionen wider. Die induzierenden elektromagnetischen Felder dringen aufgrund des Skineffekts abhängig von der Frequenz und dem spezifischen Widerstand des Untergrundes unterschiedlich tief ein.

\subsection{1 $\rho^{*}\left(z^{*}\right)$-Darstellung}

Einen guten Überblick über die vertikale Leitfähigkeitsverteilung im Meßgebiet gibt die $\rho^{*}\left(z^{*}\right)$-Verteilung. Sie kann als Anhaltspunkt für die tatsächliche eindimensionale Widerstandsverteilung unter der Meßstation dienen. Insbesondere gibt sie Aufschluß darüber, welcher Tiefenbereich mit den längsten verwendeten Perioden überhaupt aufgelöst werden kann. In Abbildung 4.1 ist ein solches Tiefen-Widerstandsprofil für die Station SEL im Osten des Meßgebiets abgebildet.

Beide Polarisationen zeigen einen sehr ähnlichen Kurvenverlauf und sind gegeneinander verschoben, wobei die Kurve der $x y$-Polarisation durchgehend die kleineren $\rho^{*}$-Werte aufweist. Ausgehend von hohen Leitfähigkeiten in Tiefen von 10 - $20 \mathrm{~km}$ wird der spezifische Widerstand mit zunehmender Tiefe größer und erreicht ein Maximum zwischen 40 - $50 \mathrm{~km}$. Danach sieht man besonders in der $y x$-Polarisation einen Abfall bis zu einem guten Leiter in etwa 80 - $100 \mathrm{~km}$ Tiefe.

Bezüglich der maximalen Eindringtiefen ist diese Darstellung für fast alle Stationen des Arrays charakteristisch. Mit den hier verwendeten Perioden im Bereich $10 \mathrm{~s}-1 \mathrm{~h}$ können 


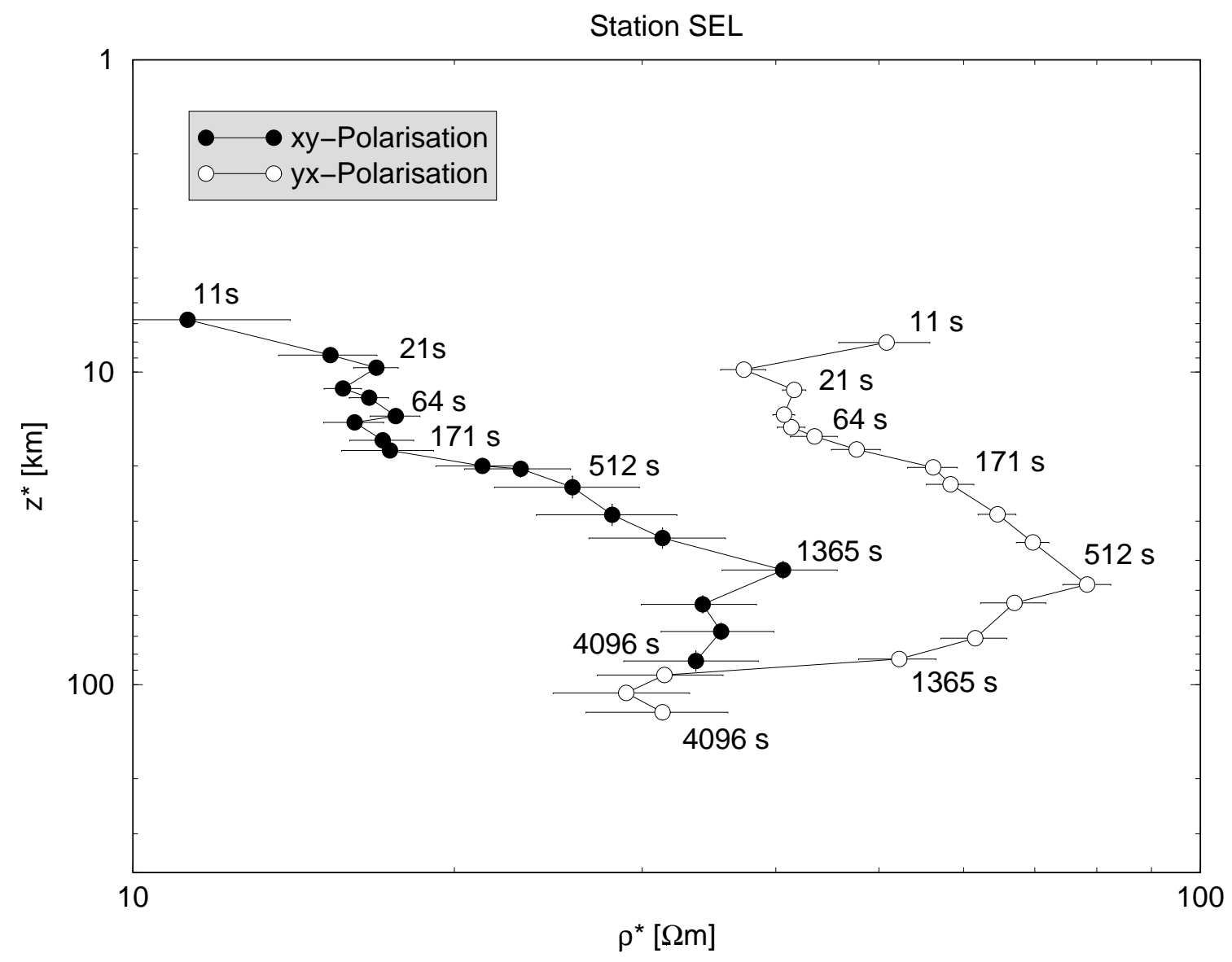

Abbildung 4.1: $\rho^{*}\left(z^{*}\right)$-Darstellung für die Station SEL. An den Datenpunkten sind teilweise die zugehörigen Perioden eingezeichnet.

somit Aussagen über die vertikale Leitfähigkeitsverteilung bis in etwa $100 \mathrm{~km}$ Tiefe, also bis in den obersten Erdmantel hinein, getroffen werden. Nur in Ausnahmefällen werden größere Eindringtiefen erreicht.

Diese finden sich ausschließlich bei den Stationen in der Nähe des Hunsrück (OSB, TRA, GOA) und liegen dort bei etwa 200 - $300 \mathrm{~km}$. Als Beispiel sind in Abbildung 4.2 die $\rho^{*}\left(z^{*}\right)$-Kurven der Station GOA dargestellt.

Die $\rho^{*}$-Werte liegen insgesamt rund eine halbe Größenordnung über denen der Station SEL, ansonsten besitzen die Kurven aber die gleichen charakteristischen Merkmale. In der $y x$-Polarisation ist wieder deutlich das Eintauchen in einen guten Leiter im obersten Erdmantel zu erkennen.

Die $\rho^{*}\left(z^{*}\right)$-Kurven der Station HIL weisen einige Unterschiede auf. Bei kurzen Perioden zeigt sich bei der $x y$-Polarisation in etwa 15 - $20 \mathrm{~km}$ Tiefe ein Minimum des Widerstandes, welches man bei SEL und GOA nicht in dieser Deutlichkeit beobachten kann.

Es fällt auf, daß bei HIL im Gegensatz zu den beiden anderen Stationen die zur $y x$ Polarisation gehörenden $\rho^{*}$-Werte niedriger sind. Die maximale Eindringtiefe liegt dabei nur bei etwa $40 \mathrm{~km}$, die Kurve bleibt gewissermaßen in einem guten Leiter in der 

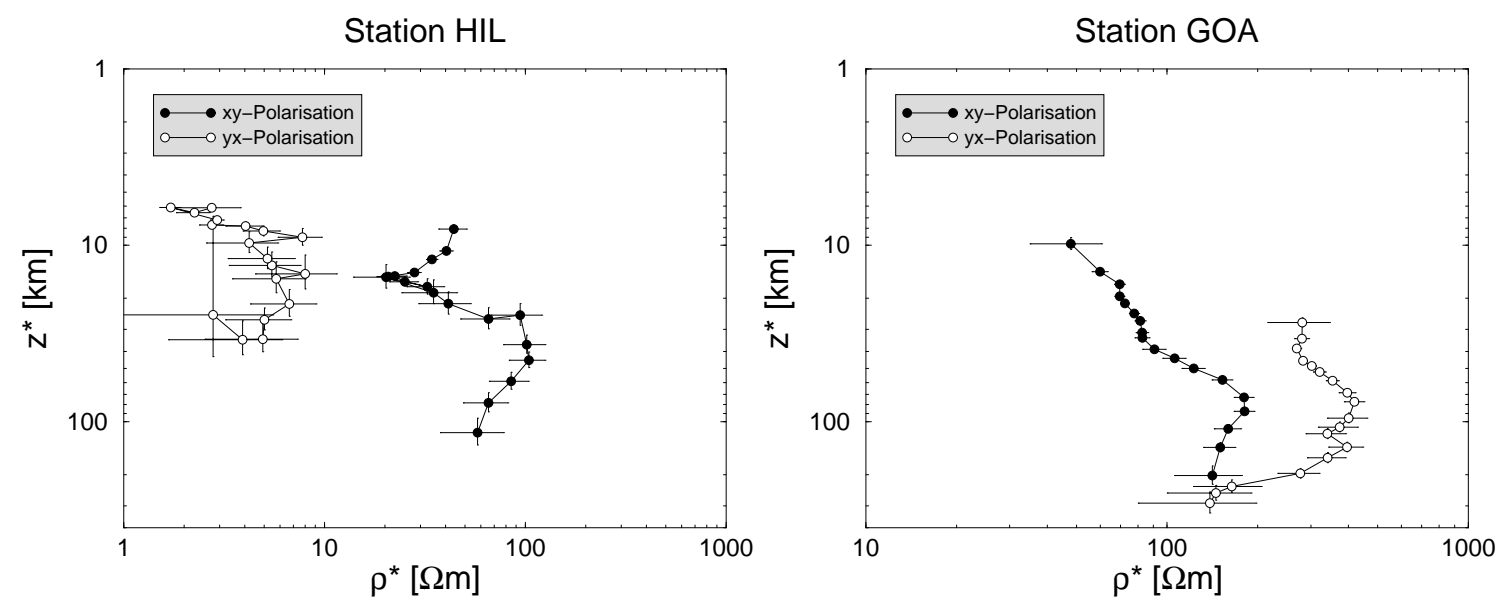

Abbildung 4.2: $\rho^{*}\left(z^{*}\right)$-Darstellung für die Stationen HIL und GOA. Die verwendeten Perioden sind mit denen in Abbildung 4.1 identisch.

Kruste stecken. Insgesamt zeigen die $\rho^{*}\left(z^{*}\right)$-Werte der $y x$-Polarisation einen sehr uneinheitlichen Verlauf. Zum Teil nehmen die $z^{*}$-Werte nicht mit wachsender Periode zu, wie es beim eindimensionalen Untergrund der Fall wäre.

Dies sind bereits deutliche Hinweise darauf, daß bei HIL eine kompliziertere, möglicherweise dreidimensionale Leitfähigkeitsverteilung vorliegt.

\subsubsection{Phase der Impedanz und scheinbarer spezifischer Widerstand}

Nachfolgend sind die Frequenzgänge der Phase und des scheinbaren spezifischen Widerstandes einiger ausgewählter Stationen abgebildet. Bei der Auswahl dieser exemplarischen Stationen wurde darauf geachtet, daß sie über das ganze Meßgebiet verteilt sind und eine gute Datenqualität besitzen (vgl. HöNIG, 1998). 
BAD

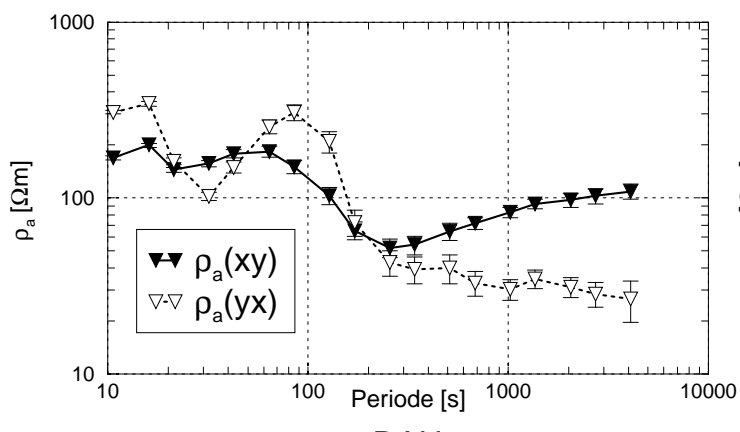

$\mathrm{DAH}$

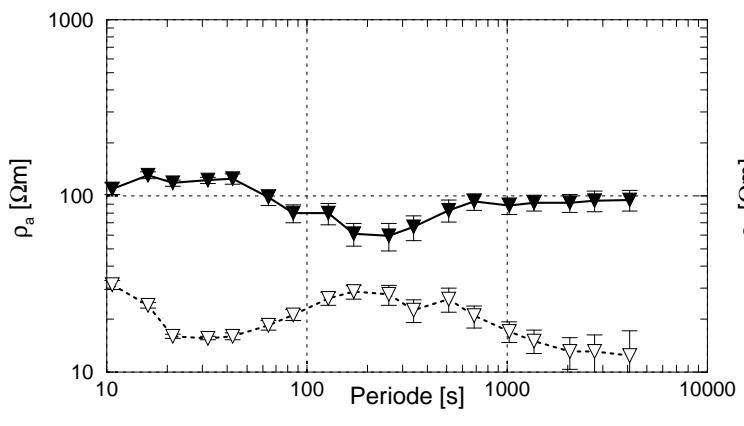

GOA

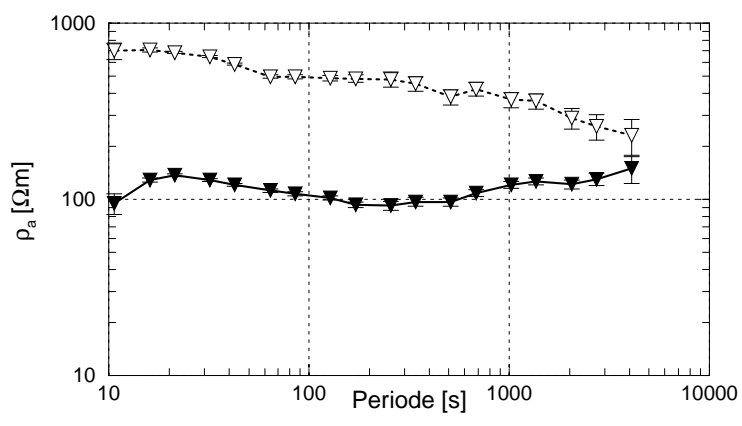

SEL

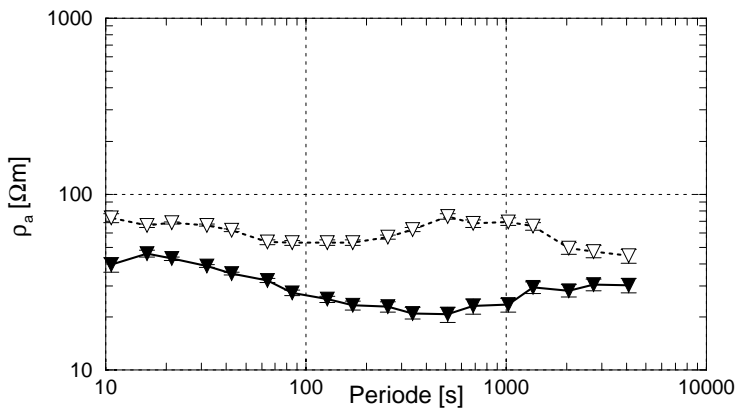

$\mathrm{BOO}$

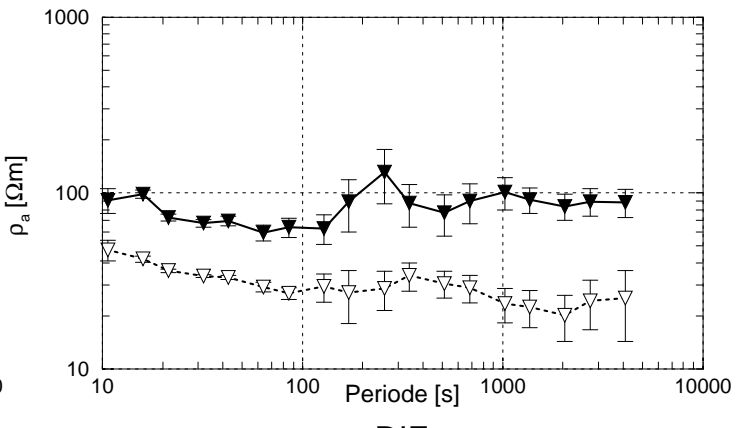

DIE

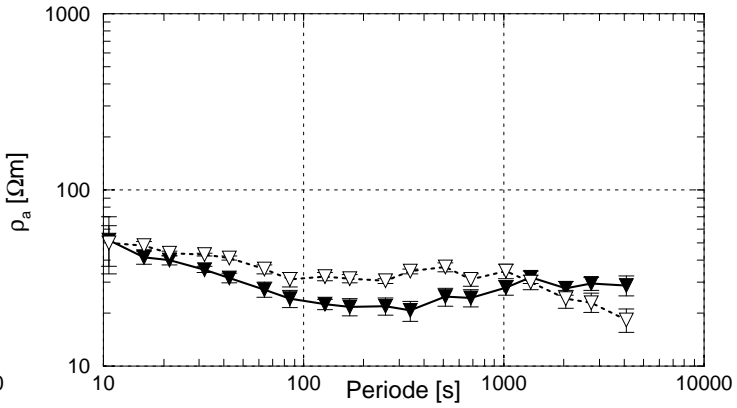

HIL

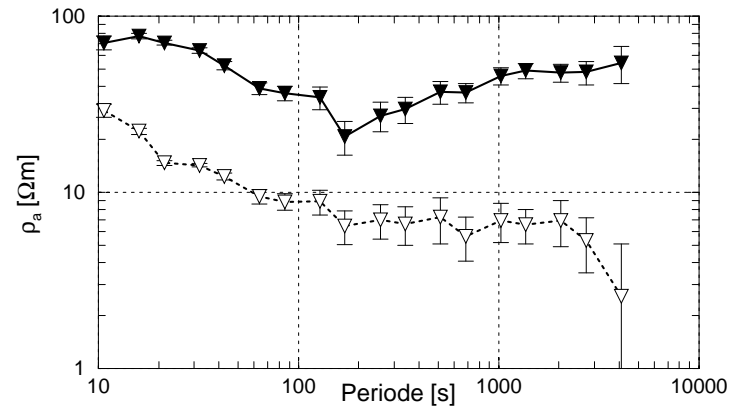

TRA

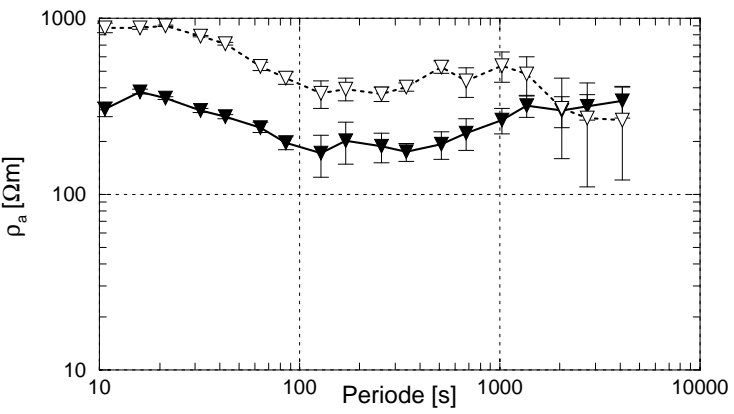

Abbildung 4.3: Frequenzgänge des scheinbaren spezifischen Widerstandes einiger ausgewählter Stationen. Man beachte die unterschiedliche Skalierung der $y$-Achse bei HIL. Die Daten sind nicht rotiert. 
BAD

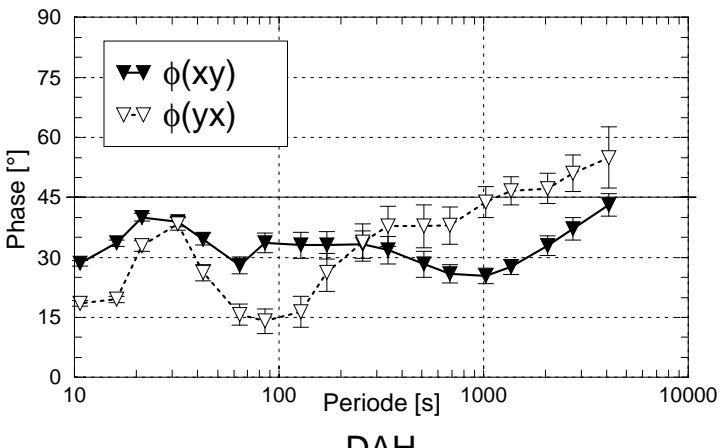

$\mathrm{DAH}$
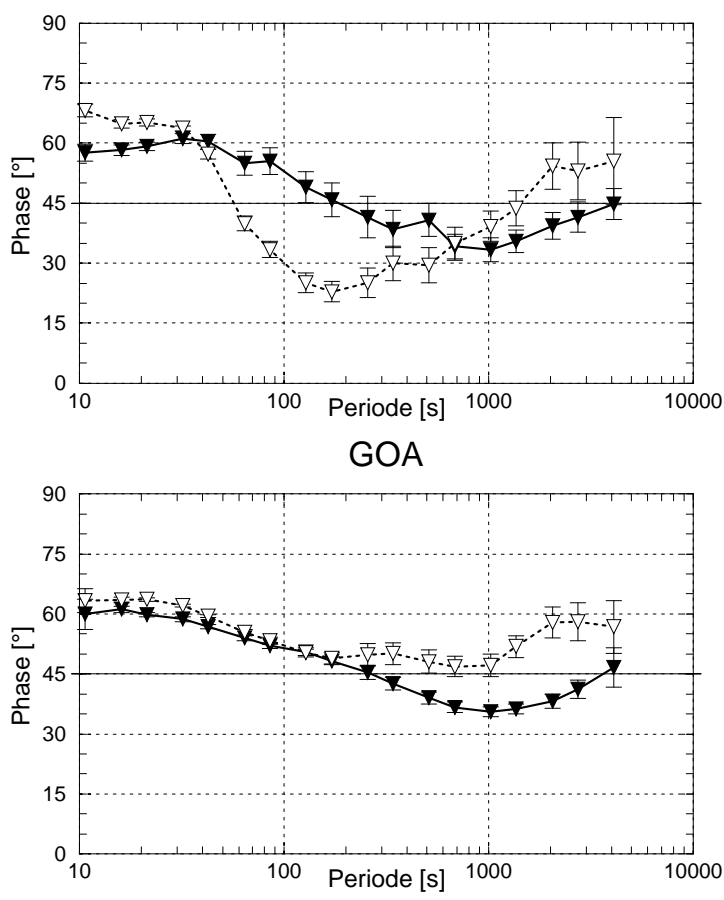

SEL

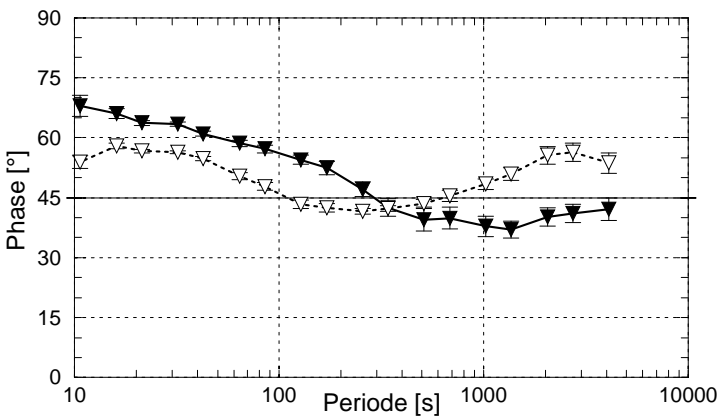

$\mathrm{BOO}$

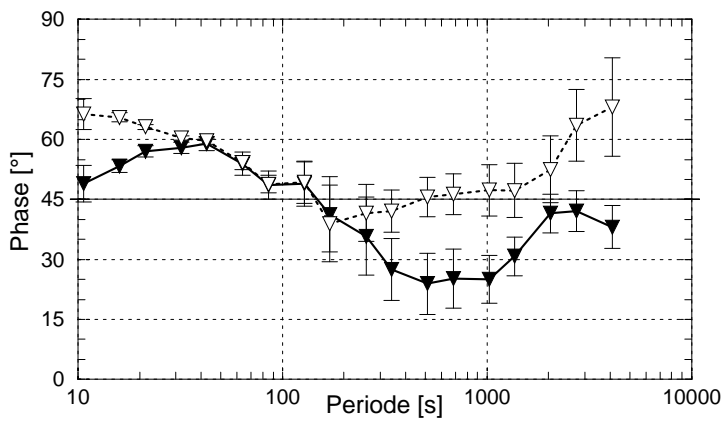

DIE

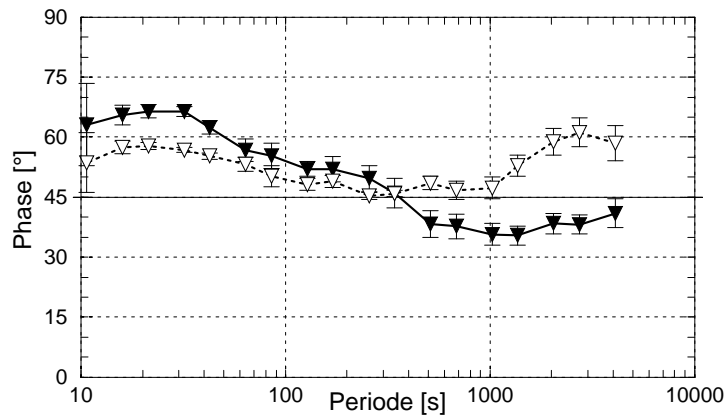

HIL

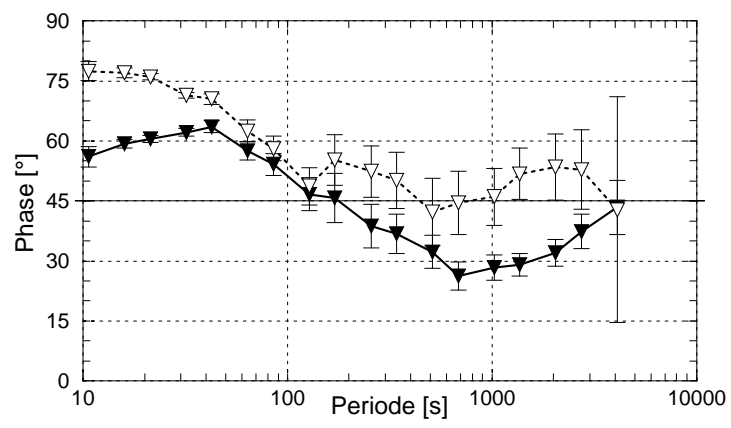

TRA

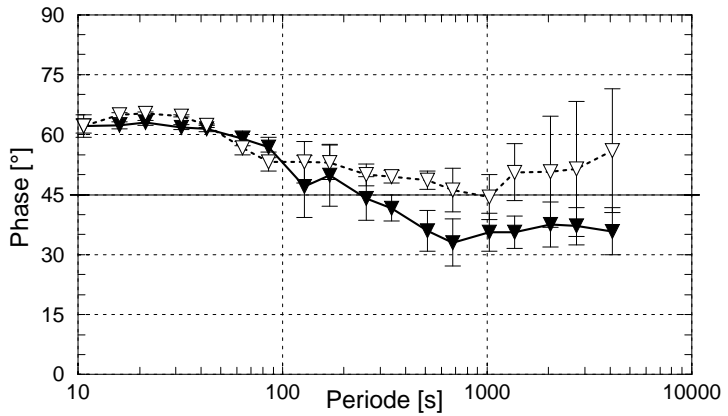

Abbildung 4.4: Frequenzgänge der Phase der Impedanz einiger ausgewählter Stationen. Die Daten sind nicht rotiert. 
Die Frequenzgänge des scheinbaren spezifischen Widerstandes lassen nur wenige Besonderheiten erkennen. Die meisten Stationen zeigen eine Aufspaltung der Polarisationen, die über den gesamten Periodenbereich relativ konstant ist. Aus diesem Grund kommt als Ursache eine statische Verzerrung der elektrischen Felder in Frage (vgl. Kapitel 1.8 und Abbildung 1.8).

Bei den Hunsrück-Stationen GOA und TRA werden die größten Widerstände beobachtet. Sie liegen dort mit 100 - $1000 \Omega$ m etwa eine Größenordnung über den anderen Stationen, deren $\rho_{a}$-Werte etwa 10 - $100 \Omega$ m betragen. Die niedrigsten Widerstände mit zum Teil weniger als $10 \Omega \mathrm{m}$ werden bei der Station HIL gemessen.

Die Stationen BAD und DIE zeigen ein etwas abweichendes Verhalten. Während BAD die deutlichste Variation des scheinbaren spezifischen Widerstandes mit der Frequenz erkennen läßt, schwankt $\rho_{a}$ bei DIE über dem gesamten Periodenbereich in beiden Polarisationen nur geringfügig zwischen 20 und $50 \Omega \mathrm{m}$.

Die Betrachtung der Phasenverläufe in Abbildung 4.4 bietet ein interessanteres Bild. Bis auf BAD zeigen alle Stationen zum größten Teil übereinstimmende Charakteristika. Im Periodenbereich bis etwa 100 - $200 \mathrm{~s}$ liegen die Phasenkurven über $45^{\circ}$, im Mittel liegt das Maximum bei rund $60^{\circ}$. Die beiden Polarisationen spalten zum Teil auf, jedoch nicht in einheitlicher Form. Sehr deutlich ist dies bei Station HIL zu beobachten, wo die Phase der $y x$-Polarisation bis auf über $75^{\circ}$ ansteigt. Beide Polarisationen liegen dann im Bereich zwischen $200 \mathrm{~s}$ und $500 \mathrm{~s}$ nahe bei $45^{\circ}$.

Besonders bemerkenswert ist das einheitliche Verhalten aller Stationen bei längeren Perioden. Die Polarisationen spalten wieder auf, wobei die Kurve der $x y$-Polarisation zunächst abfällt, ein Minimum von $25^{\circ}-35^{\circ}$ um 1000 s durchläuft und danach wieder ansteigt, jedoch immer unter $45^{\circ}$ bleibt. Im Gegensatz dazu steigen die Phasen der $y x$-Polarisation an und erreichen Maxima von etwa $60^{\circ}$ bei Perioden von 2000 - 4000 s. Besonders ausgeprägt und in einheitlicher Form findet man das beschriebene Verhalten bei den Stationen, die im Osten des Meßgebiets liegen (SEL, DIE, GOA).

Das gleichartige Verhalten der Phasen bei fast allen Stationen deutet bereits auf den Einfluß großräumiger Leitfähigkeitsanomalien oder anisotroper Strukturen hin.

\subsubsection{Bestimmung von Vorzugsrichtungen}

Um Informationen über Streichrichtungen zweidimensionaler Strukturen zu erhalten, bietet sich eine Analyse der Rotationswinkel nach SWIFT (Gleichung 2.19) oder BAHR (Gleichung 2.20) an.

Bereits bei der Betrachtung der scheinbaren spezifischen Widerstände wurde darauf hingewiesen, daß an einigen Stationen die elektrischen Felder wahrscheinlich galvanisch verzerrt sind ${ }^{1}$. In diesem Fall lassen sich durch die Analyse der Swiftwinkel $\alpha_{S}$ keine Informationen über Vorzugsrichtungen induktiv wirksamer Strukturen gewinnen, da die gefundene Richtung im wesentlichen durch quasi-statische Effekte lokaler Störkörper be-

\footnotetext{
${ }^{1}$ Untersuchungen von HöNIG (1998) bestätigen dies.
} 
stimmt wird.

Durch die Betrachtung des phasensensitiven Rotationswinkels $\alpha_{B}$ können diese Probleme vermieden werden.

Bei der Bestimmung und Interpretation von Rotationswinkeln muß jedoch sichergestellt sein, daß eine zweidimensionale Interpretation gerechtfertigt ist. Aus diesem Grund ist in Abbildung $4.5 \alpha_{B}$ zusammen mit der phasensensitiven Schiefe $\eta$ nach Gleichung 2.13 für mehrere über das Array verteilte Stationen dargestellt.

Man erkennt einen deutlichen Frequenzgang des Rotationswinkels. Im Bereich kurzer Perioden unter 100 - 200 s schwankt der Winkel zum größten Teil zwischen $15^{\circ}$ und $45^{\circ}$, dann folgt ein Übergangsbereich, und für lange Perioden $>1000 \mathrm{~s}$ liegt der Winkel im Bereich um $90^{\circ} / 0^{\circ 2}$.

Im unteren Teilbild ist die phasensensitive Schiefe $\eta$ abgebildet. Dabei sind für drei Periodenbereiche die jeweiligen Mittelwerte berechnet worden. In der Regel liegen die Werte bei allen Stationen zwischen 0.2 und 0.25 und nur in Ausahmefällen über 0.3. Dies bedeutet, daß das Superpositionsmodell (Gleichung 2.8) näherungsweise anwendbar und eine regionale zweidimensionale Interpretation gerechtfertigt ist.

Interpretiert man den einheitlichen Verlauf der Rotationswinkel zusammen mit dem Frequenzverhalten der Phasen (Abbildung 4.4), so liegt die Vorstellung nahe, daß hier zwei Leitfähigkeitsstrukturen mit unterschiedlicher Orientierung überlagert sind. In der Kruste existiert eine Zone mit einer Vorzugsrichtung in nordost-südwestlicher Richtung, in größeren Tiefen liegt vermutlich eine zweidimensionale Struktur mit Streichrichtung in West-Ostrichtung vor.

\footnotetext{
${ }^{2}$ Der Rotationswinkel wird üblicherweise im Bereich von $0^{\circ}$ bis $90^{\circ}$ bestimmt. Werte, die knapp über oder unter den beiden Eckwerten liegen, werden um $90^{\circ}$ verschoben. Zur Interpretation der gefundenen Streichrichtung muß deshalb immer eine zusätzliche Größe verwendet werden, die die Zuordnung zu E- oder B-Polarisation gestattet (vgl. Kapitel 2.3.1).

Eine Betrachtung der Phasenaufspaltung (Abbildung 4.15) zeigt, daß die gefundenen Winkel in diesem Fall zur gleichen Streichrichtung gehören.
} 

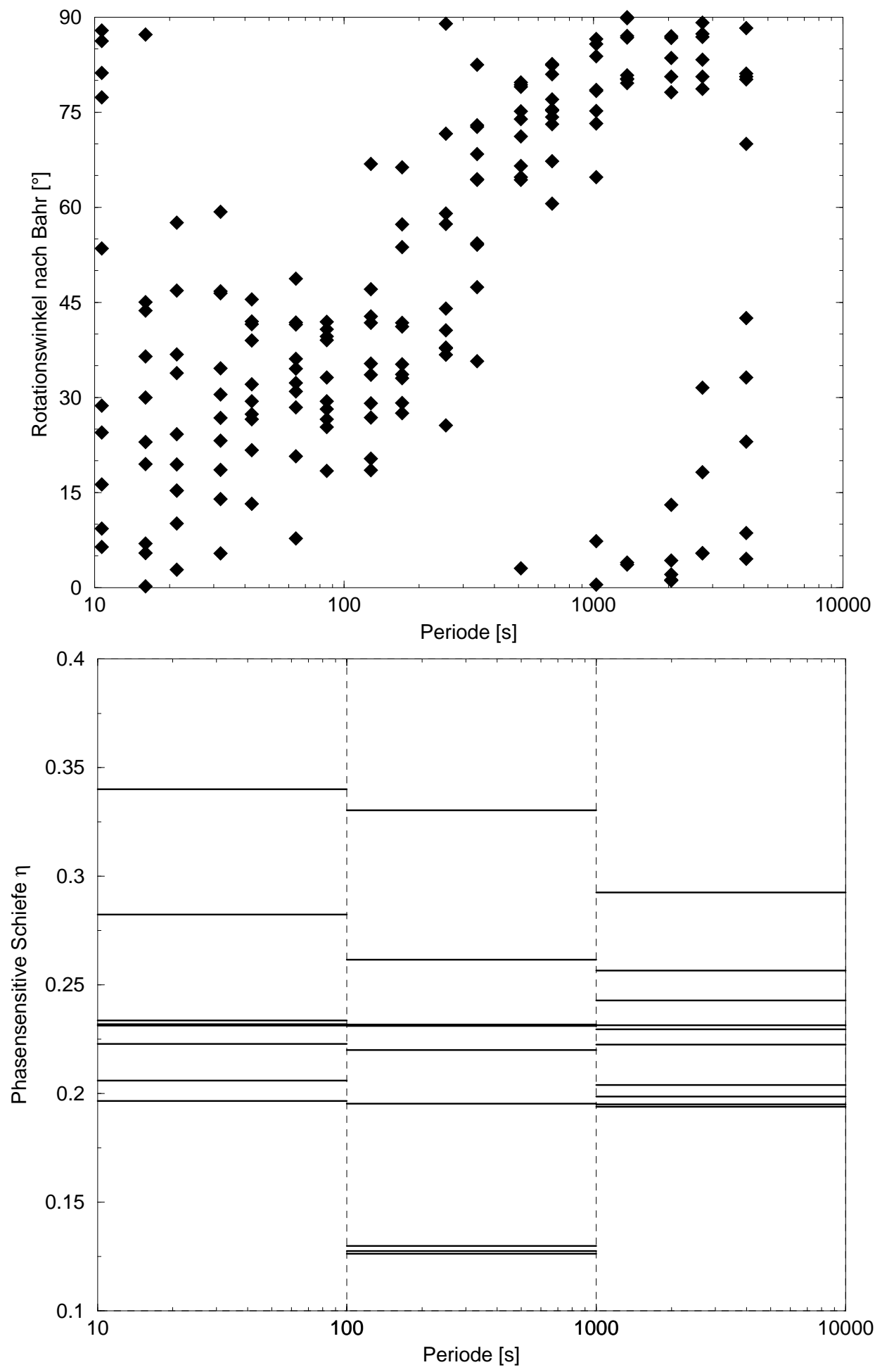

Abbildung 4.5: Frequenzgang des phasensensitiven Rotationswinkels $\alpha_{B}$ und der phasensensitiven Schiefe $\eta$ für 10 Stationen: AHR, ALT, BAD, COC, DIE, GOA, HON, LAH, SEL. 


\subsection{Daten zur Kruste}

\section{Bemerkungen zur Wahl der Referenzstation}

Grundsätzlich wurde für alle magnetischen Übertragungsfunktionen zunächst Station DIE als Referenzstation gewählt.

Bei den Darstellungen der rotationsinvarianten Maßzahlen (Abbildung 4.6) und der Perturbationspfeile (Abbildung 4.11) wurde deren Einfluß auf die Übertragungsfunktionen der Feldstationen mit dem in Kapitel 2.4.2 vorgestellten Verfahren beseitigt. Das zugehörige lokale Modell für Station DIE wird in Kapitel 5.5 ausführlich beschrieben.

Um den Vergleich mit späteren Modellrechnungen zu vereinfachen, wird bei den übrigen Darstellungen von einzelnen Elementen der Perturbationstensoren als Bezugsstation weiterhin die Station DIE verwendet. Dies erscheint auch im Hinblick auf die Überlegungen in Kapitel 2.4.1 (Beispiel 2) gerechtfertigt.

\subsubsection{Rotationsinvariante Maßzahlen des Perturbationstensors}

Um zunächst einen Überblick über die laterale Struktur der Kruste zu bekommen, werden zwei rotationsinvariante Maßzahlen betrachtet, die in Kapitel 2.2.2 eingeführt wurden und sich als besonders geeignet zur Abbildung der räumlichen Leitfähigkeitsverteilung erwiesen haben. In Abbildung 4.6 ist eine flächenhafte Darstellung von $\operatorname{Re}\left\{T_{1} / 2\right\}$ und $\kappa_{G 1}$ für die Periode $171 \mathrm{~s}$ abgebildet. Die Eindringtiefe dieser Periode liegt im Bereich der mittleren Kruste.

In den Darstellungen treten zwei Anomalien besonders deutlich hervor. Zum einen erkennt man in $\operatorname{Re}\left\{T_{1} / 2\right\}$ ein lokales Maximum in der Westeifel (DAH, HON, HIL). Diese Struktur zeigt sich ebenfalls bei einer Auftragung von $\kappa_{G 1}$.

Außerdem erscheint noch eine zweite Anomalie im Süden des Meßgebiets bei den Stationen SAA, IDA und BKR. Sie verläuft südlich des Hunsrück und ist ungefähr in Richtung des variszischen Streichens orientiert. Besonders bemerkenswert ist, daß sich diese Struktur in $\kappa_{G 1}$ nicht abzeichnet. Dies bedeutet, daß sie wahrscheinlich durch ein zweidimensionales Modell erklärt werden kann. Da beide Maßzahlen im Bereich der Westeifel nicht verschwinden, ist davon auszugehen, daß die Leitfähigkeitsverteilung hier eher dreidimensionaler Natur ist. 


\section{$\operatorname{Re}\left\{T_{1} / 2\right\}(171 s)$}
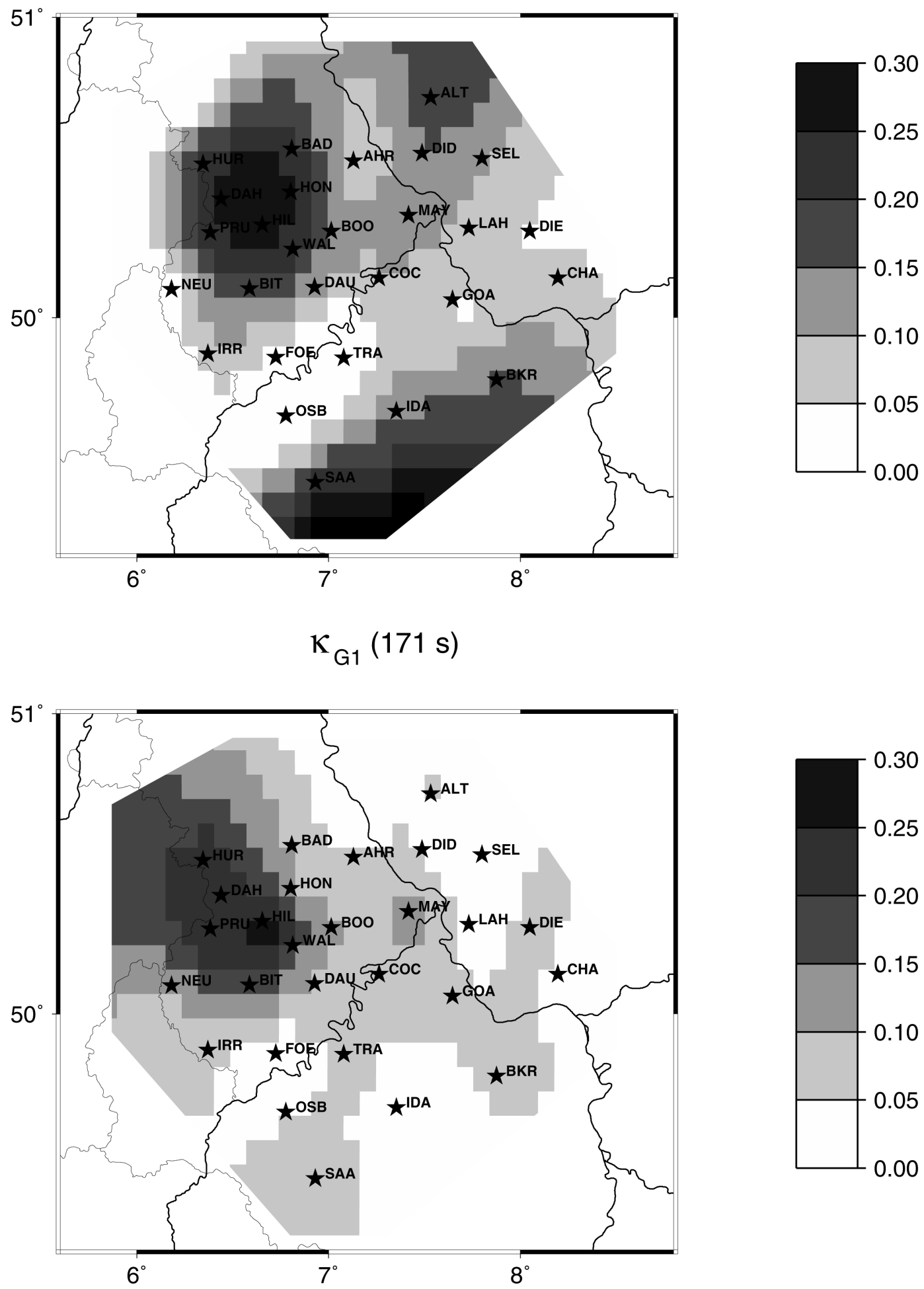

Abbildung 4.6: Flächenhafte Darstellung der Rotationsinvarianten des Perturbationstensors $\operatorname{Re}\left\{T_{1} / 2\right\}$ und $\kappa_{G 1}$ bei einer Periode von $171 \mathrm{~s}$. 


\subsubsection{Magnetische Übertragungsfunktionen}

In Abbildung 4.7 sind die Frequenzgänge von $d_{D}$ für die Stationen HON im Gebiet der nordwestlichen Anomalie und BKR am Südrand des Rheinischen Schiefergebirges gezeigt. Dabei wurden die Daten so rotiert, daß die Summe der Nebendiagonalelemente $\left|h_{D}\right|+\left|d_{H}\right|$ minimiert wurde und $\left|d_{D}\right|>\left|h_{H}\right|$ war. Für Station HON ergab sich ein Winkel von $-17^{\circ}$ und für Station BKR ein Winkel von $76^{\circ}$. Diese Rotationswinkel beziehen sich jeweils auf die Periode, bei der das Maximum im Frequenzgang der horizontalen Übertragungsfunktionen erreicht wird.

Im Frequenzgang von $\operatorname{Re}\left\{d_{D}\right\}$ zeigen sich bei beiden Stationen ausgeprägte Maxima mit Amplituden von 0.37 (HON) und 0.49 (BKR). Sie werden bei Perioden von 256 s, bzw. 683 s erreicht.
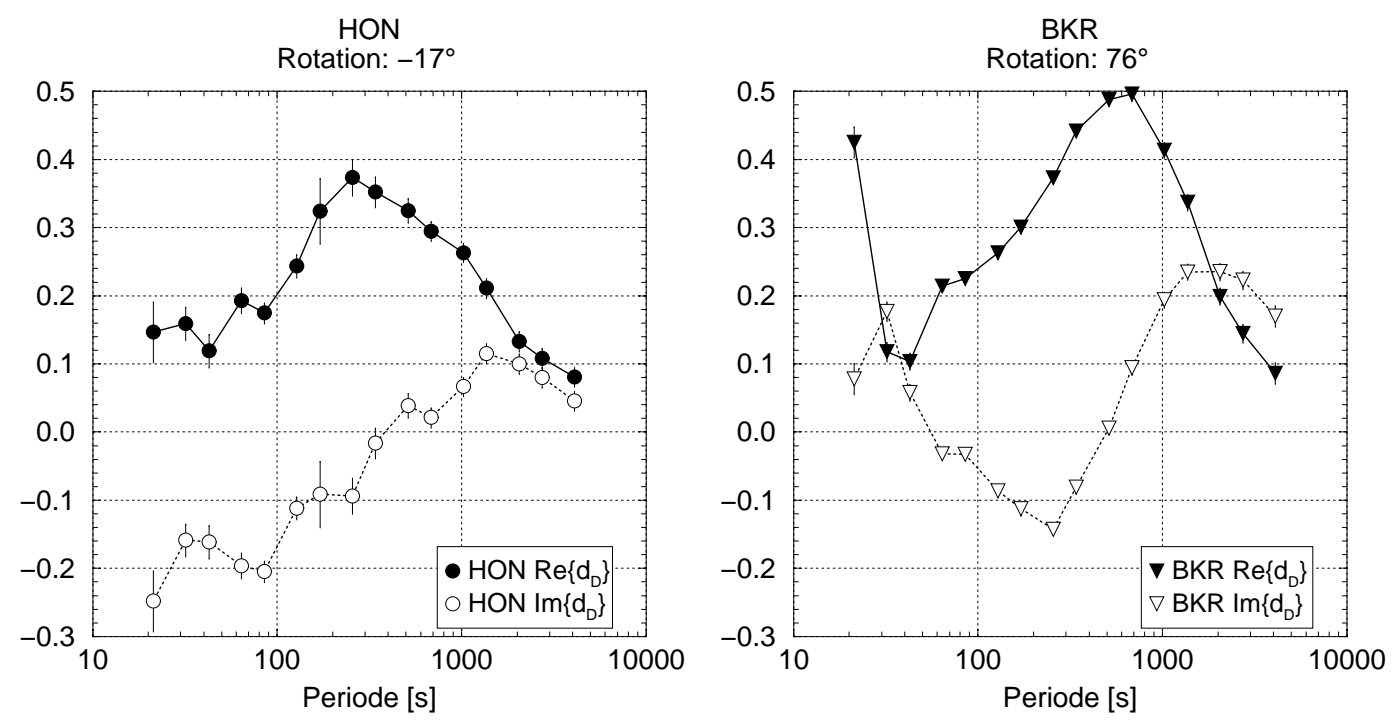

Abbildung 4.7: Frequenzgänge von $d_{D}$ der Stationen HON und BKR in rotierten Koordinaten.

Nimmt man an, daß als Ursache annähernd zweidimensionale Leitfähigkeitsstrukturen in Frage kommen, so stehen beide offenbar fast senkrecht aufeinander. Dabei hat die nordwestliche Anomalie im wesentlichen eine Nord-Süd streichende Komponente, die südliche ist entsprechend eher in West-Ostrichtung orientiert.

Beim idealen zweidimensionalen Fall sollte der Perturbationstensor inklusive der vertikalen Übertragungsfunktionen im richtigen Koordinatensystem ( $x^{\prime}$-Richtung $\widehat{=}$ Streichrichtung) nur die beiden Elemente $d_{D}$ und $z_{D}$ enthalten. In Tabelle 4.1 sind die Beträge aller Elemente für die Periode $256 \mathrm{~s}$ und $683 \mathrm{~s}$ zusammengestellt.

Bei beiden Stationen ist $d_{D}$ das mit Abstand größte Element, bei HON liegen etwa $36 \%$ der Information in den übrigen drei Elementen, bei BKR sind es $42 \%$.

Bemerkenswert ist, daß bei HON $\left|z_{H}\right|$ keineswegs verschwindet, sondern sogar größer als $\left|z_{D}\right|$ ist. Während man zur Erklärung der horizontalen Übertragungsfunktionen von HON und BKR noch näherungsweise eine zweidimensionale Modellvorstellung heran- 


\begin{tabular}{|l|l|l|}
\hline & $\begin{array}{l}\text { HON } \\
256 \mathrm{~s} \\
\text { Rotation: }-17^{\circ}\end{array}$ & $\begin{array}{l}\text { BKR } \\
683 \mathrm{~s} \\
\text { Rotation: } 76^{\circ}\end{array}$ \\
\hline$\left|h_{H}\right|$ & 0.1004 & 0.1030 \\
\hline$\left|h_{D}\right|$ & 0.0209 & 0.1069 \\
\hline$\left|d_{H}\right|$ & 0.0952 & 0.1520 \\
\hline$\left|d_{D}\right|$ & 0.3853 & 0.5047 \\
\hline$\left|z_{D}\right|$ & 0.1104 & 0.1178 \\
\hline$\left|z_{H}\right|$ & 0.1417 & 0.0484 \\
\hline
\end{tabular}

Tabelle 4.1: Elemente des Perturbationstensors der Stationen HON und BKR in rotierten Koordinaten bei $256 \mathrm{~s}$ und $683 \mathrm{~s}$.

ziehen konnte, paßt das Verhalten der vertikalen Übertragungsfunktionen bei HON nicht in dieses Bild.

Mögliche Hinweise zur Klärung dieses Befundes ergeben sich aus Abbildung 4.8. Hier sind die Übertragungsfunktionen $\operatorname{Re}\left\{d_{D}\right\}$ und $\operatorname{Re}\left\{z_{D}\right\}$ für Stationen auf einem Profil dargestellt, welches senkrecht zur vermuteten Streichrichtung der südlichen Anomalie verläuft. Die Daten sind für die Periode 683 s dargestellt und um $76^{\circ}$ rotiert.

Man erkennt, daß das Maximum im $\operatorname{Re}\left\{d_{D}\right\}$ lokal auf die südlichste Station IDA begrenzt ist, der Einfluß des anomalen vertikalen Magnetfeldes sich dagegen über die gesamte Profillänge erstreckt . Es ist ein Vorzeichenwechsel zu beobachten, dessen Nulldurchgang etwa bei Profilkilometer 70 zwischen COC und TRA liegt. Er befindet sich allerdings nicht unter dem größten Effekt im horizontalen Magnetfeld. Wahrscheinlich wird das Maximum und das Minimum im $\operatorname{Re}\left\{z_{D}\right\}$ über dem Profil noch nicht erreicht.

Diese Überlegungen machen deutlich, daß die Station HON offenbar durch das vertikale Magnetfeld der südlichen Leitfähigkeitsanomalie noch in beträchtlichem Maße beeinflußt wird. Es ist somit davon auszugehen, daß das Verhalten der vertikalen Übertragungsfunktionen $z_{H}$ und $z_{D}$ bei HON die überlagerten Effekte zweier Anomalien widerspiegelt. Da deren Streichrichtungen nahezu senkrecht aufeinander stehen ${ }^{3}$, können die Einflüsse beider Strukturen gut unterschieden werden, indem man beide Polarisationen getrennt interpretiert.

Das schnelle Abklingen des $\operatorname{Re}\left\{d_{D}\right\}$ über dem Profil zeigt, daß ein ähnlicher Effekt im horizontalen Magnetfeld nicht zu erwarten ist. Dies wird auch durch Modellrechnungen von KURAS (1998) bestätigt.

In Abbildung 4.9 ist eine flächenhafte Darstellung des $\operatorname{Re}\left\{d_{D}\right\}$ und des $\operatorname{Re}\left\{z_{D}\right\}$ bei der Periode 256 s gezeigt. Die räumliche Ausdehnung der Anomalie ist besonders bei der Auftragung des $\operatorname{Re}\left\{d_{D}\right\}$ gut abzuschätzen. Das lokale Maximum von 0.38 wird bei Stati-

\footnotetext{
${ }^{3}$ Diese Tatsache vereinfacht die Interpretation. Allerdings ist zu beachten, daß der lokal nicht erklärte Anteil $\operatorname{Re}\left\{z_{H}\right\}$ von HON im Koordinatensystem der nordwestlichen Anomalie gerade einem Anteil von $\operatorname{Re}\left\{z_{D}\right\}$ im Koordinatensystem der südlichen Anomalie entspricht.
} 


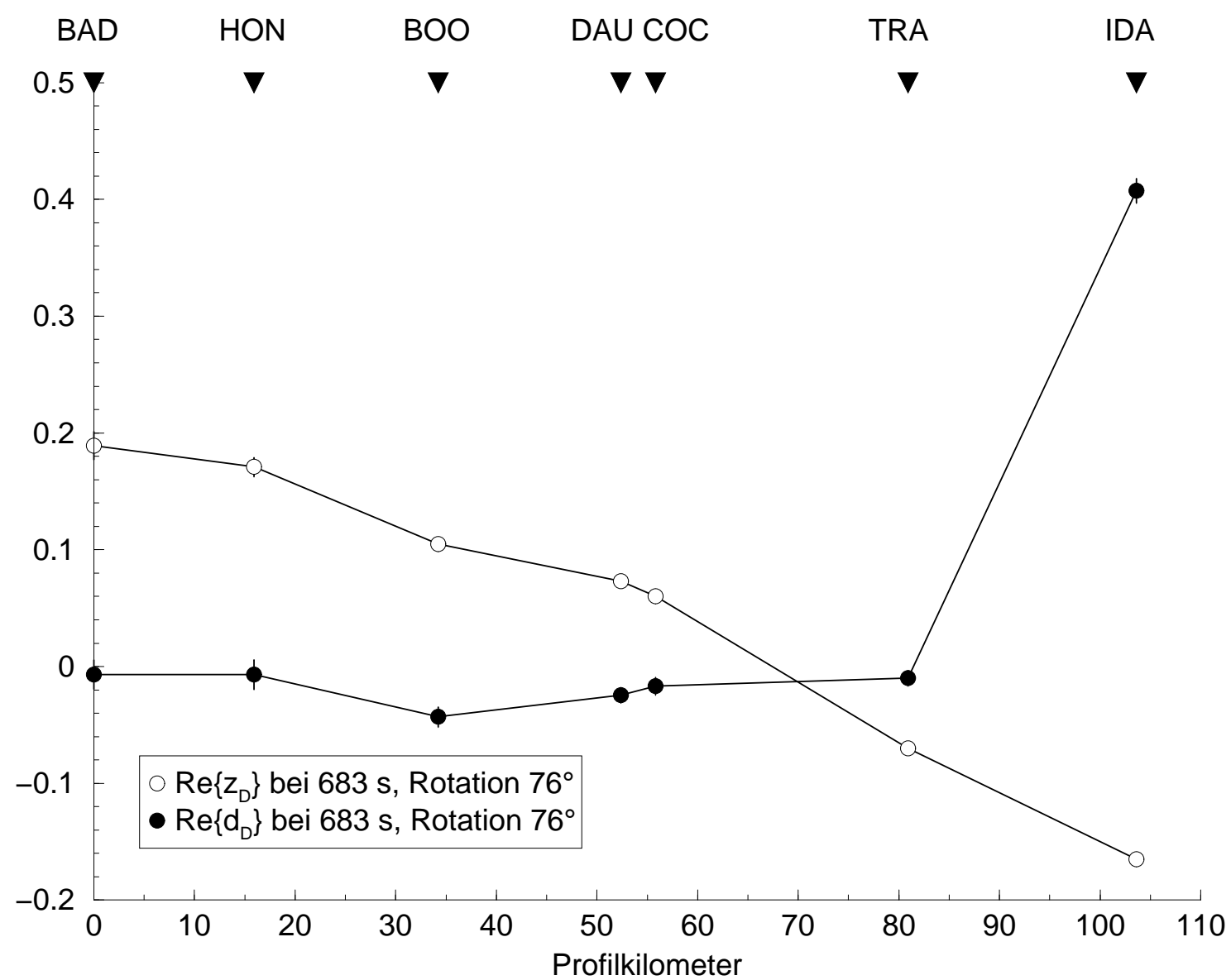

Abbildung 4.8: Darstellung von $\operatorname{Re}\left\{d_{D}\right\}$ und $\operatorname{Re}\left\{z_{D}\right\}$ über einem Profil von Station BAD im Norden bis Station IDA südlich des Hunsrück. Die Daten sind um $76^{\circ}$ rotiert (Streichrichtung der südlichen Anomalie) und für die Periode 683 s dargestellt.

on HIL gemessen. Die Halbwertsbreite beträgt in West-Ostrichtung etwa $35 \mathrm{~km}$, in NordSüdrichtung kann sie nicht sicher abgeschätzt werden, da die Stationsbedeckung im Norden nicht ausreicht.

Bei der Darstellung des $\operatorname{Re}\left\{z_{D}\right\}$ fällt auf, daß kein deutlicher Vorzeichenwechsel von West nach Ost auftritt. Dennoch ist zu erkennen, daß die Ausdehnung des vertikalen Magnetfeldes im Vergleich zur südlichen Struktur räumlich begrenzter ist. Dies deutet bereits darauf hin, daß die Ursache des anomalen Feldes in der Westeifel viel oberflächennaher liegt als im Süden. 


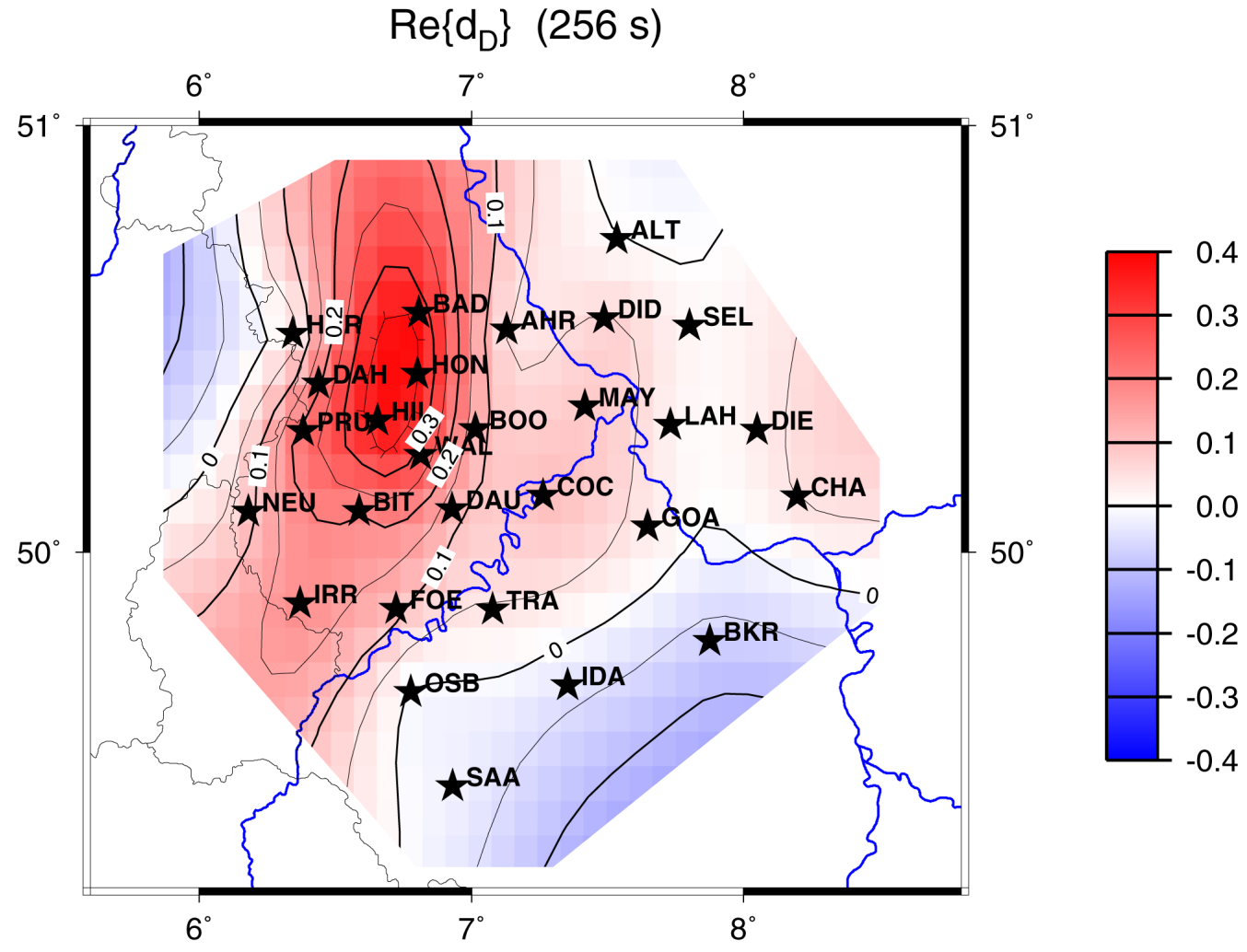

$\operatorname{Re}\left\{z_{D}\right\} \quad(256 s)$

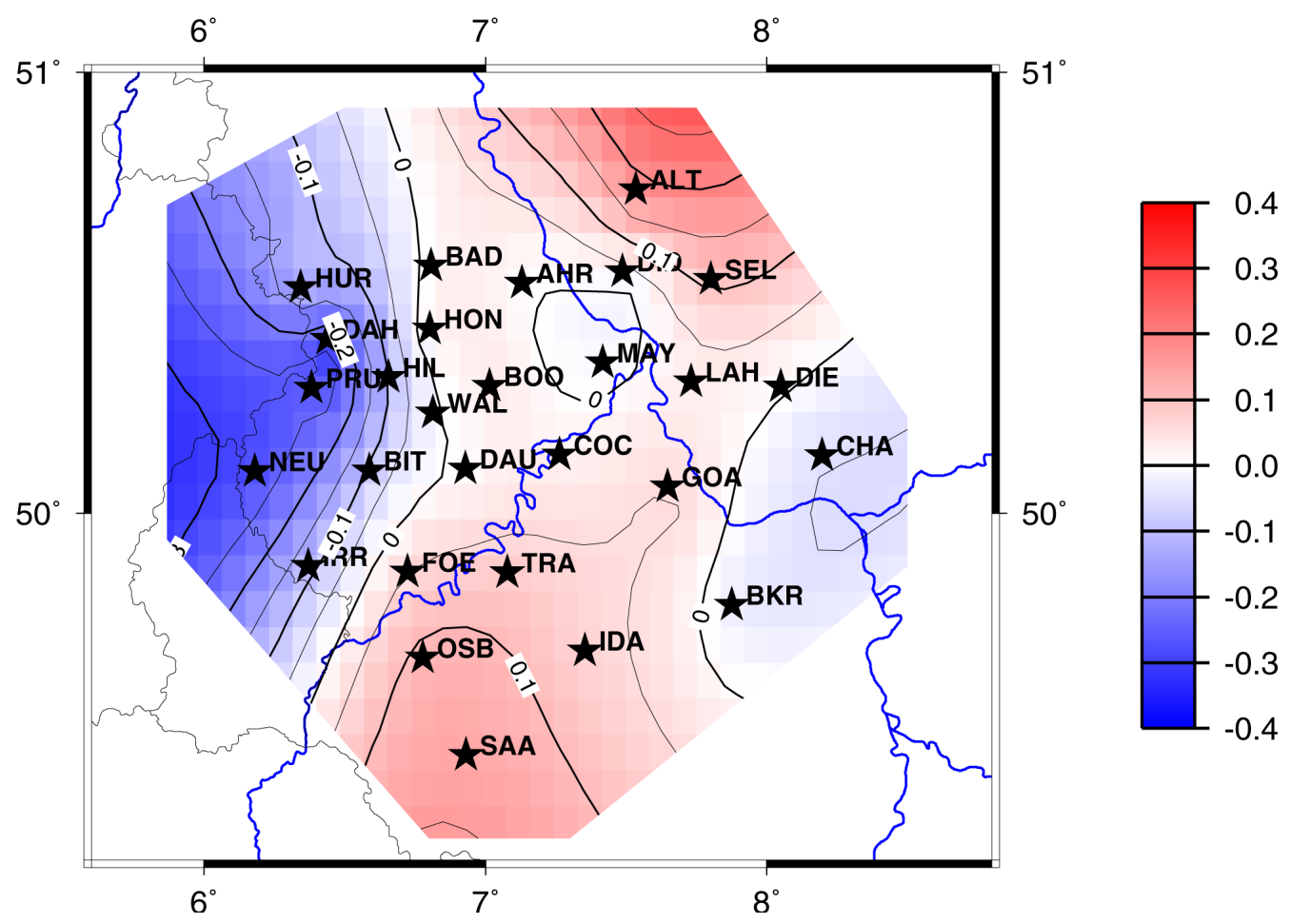

Abbildung 4.9: Darstellung von $\operatorname{Re}\left\{d_{D}\right\}$ und $\operatorname{Re}\left\{z_{D}\right\}$ für alle Stationen bei einer Frequenz von 256 s. Die Daten sind nicht rotiert. 


\subsubsection{Lokale Induktionspfeile und Perturbationspfeile bei $256 \mathrm{~s}$}

Auch die Verteilung der lokalen Induktionspfeile (Abbildung 4.10) wird hauptsächlich von den bereits besprochenen Leitfähigkeitsanomalien geprägt.

Lokale Induktionspfeile bei $256 \mathrm{~s}$

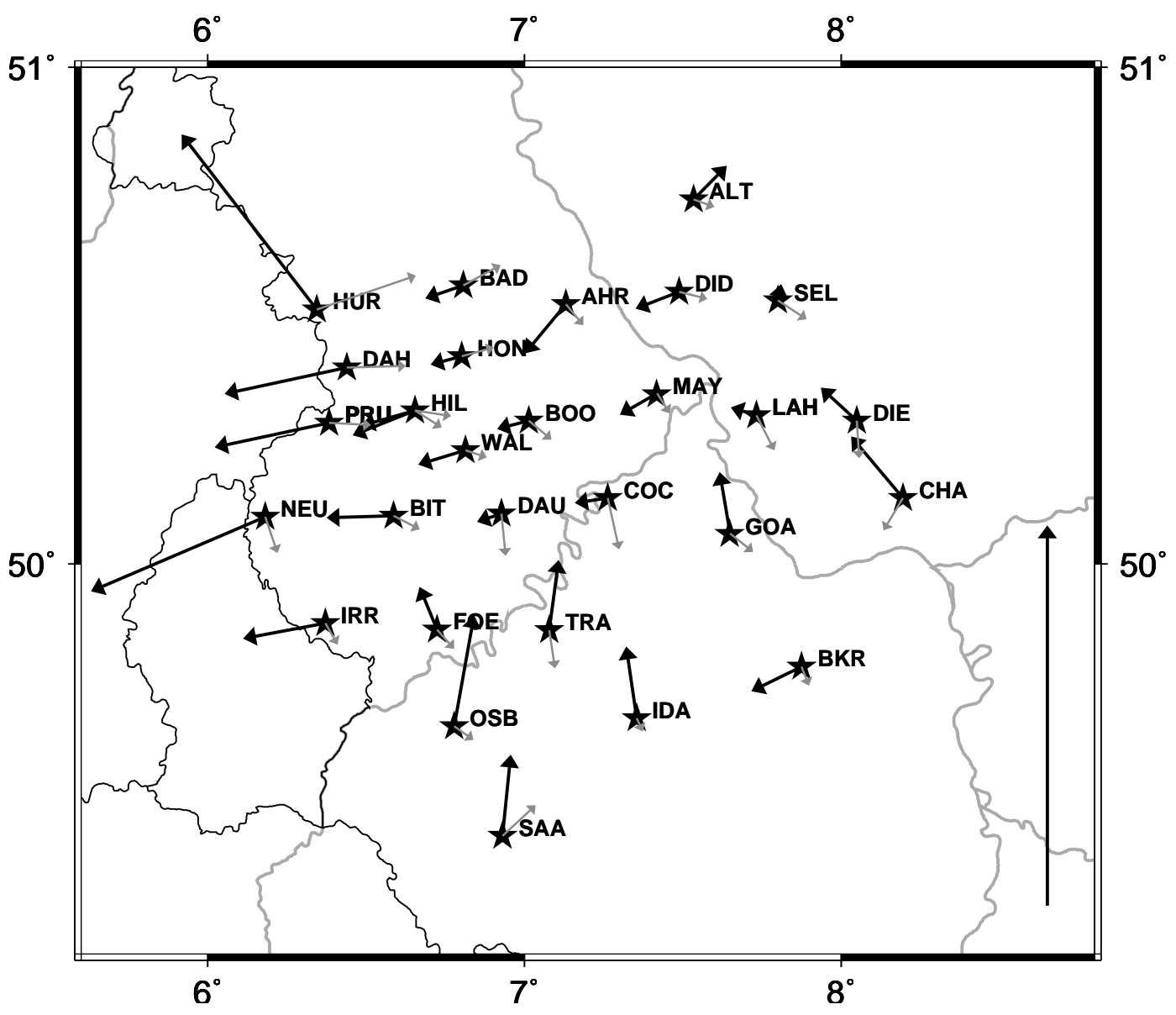

Abbildung 4.10: Flächenhafte Darstellung der lokalen Induktionspfeile für alle Stationen bei einer Periode von 256 s. Der eingezeichnete Referenzpfeil hat die Länge eins. Die Realpfeile sind schwarz dargestellt.

Die Realpfeile der westlichen Stationen HUR, DAH, PRU und NEU weisen vom Gebiet der Anomalie fort. Sie erreichen eine Länge von 0.3 - 0.5, wobei der Realpfeil bei HUR eine große Komponente in Nordrichtung besitzt. Dies könnte ein Hinweis auf die Begrenzung der Anomalie in nördlicher Richtung sein.

Bei den weiter östlich liegenden Stationen wird kein Umklappen der Pfeile beobachtet, wie es für eine zweidimensionale Struktur typisch wäre. Im wesentlichen zeigen die Pfeile dort ebenfalls in westliche Richtung, allerdings mit deutlich geringeren Längen von 0.1 - 0.2 . Bei den östlichsten Stationen haben die Indukionspfeile etwa die gleiche Länge, zeigen aber nicht mehr einheitlich in dieselbe Richtung. 
In Übereinstimmung mit der West-Ost streichenden Leitfähigkeitsanomalie weisen die Realpfeile südlich der Mosel im wesentlichen nach Norden.

In Abbildung 4.11 sind alle horizontalen magnetischen Übertragungsfunktionen für $683 \mathrm{~s}$ in Form von Perturbationspfeilen dargestellt. Hier wird die Entkopplung beider Anomalien im anomalen horizontalen Magnetfeld besonders gut sichtbar. Im oberen Teilbild fällt die scharfe Begrenzung des anomalen Bereiches auf den südlichen Teil des Meßgebiets auf. Die Realpfeile $p$ besitzen bei den drei südlichsten Stationen SAA, IDA, BKR eine Länge von fast 0.6 und stehen senkrecht zur Richtung des variszischen Streichens. Die Länge der übrigen Pfeile liegt zum Teil mehr als eine Größenordnung darunter.

Die Pfeile $q$ im unteren Bild werden überwiegend von der Anomalie in $d_{D}$ bestimmt. Durch die gemeinsame Auftragung aller Elemente des Perurbationstensors werden weitere Einzelheiten sichtbar. Während die Realpfeile im nördlichen Teil in Richtung Osten zeigen, klappen sie bei den südlichen Stationen langsam nach Südosten um. Da der Einfluß der Referenzstation entfernt wurde, kann durch eine Rotation der Pfeile um $90^{\circ}$ gegen den Uhrzeigersinn direkt die Richtung des anomalen Stromflusses bestimmt werden. Das deutet darauf hin, daß die anomale Leitfähigkeitsstruktur im Süden in Richtung Westen abknickt. 
$p(683 s)$
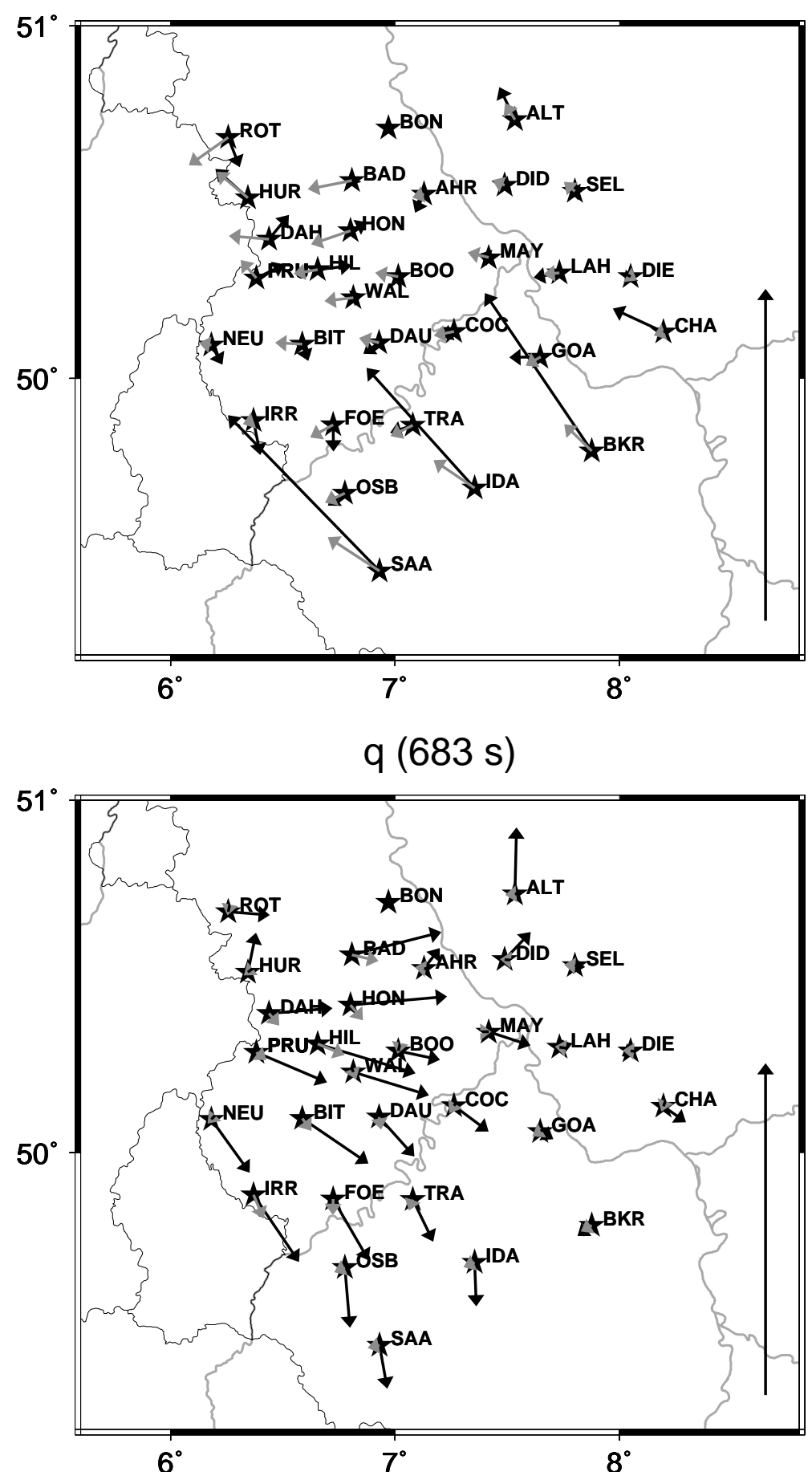

Abbildung 4.11: Flächenhafte Darstellung der Perturbationspfeile bei einer Periode von 683 s für alle Stationen. Der eingezeichnete Referenzpfeil hat die Länge eins. Die Realpfeile sind schwarz dargestellt. 
Die bisher vorgestellten Ergebnisse beschränken sich auf die Analyse des magnetischen Feldes. Im folgenden Kapitel werden einige Resultate der magnetotellurischen Methode gezeigt, bei der zusätzlich das elektrische Feld verwendet wird.

\subsection{4 $\rho^{*}\left(z^{*}\right)$-Darstellung und Phasenaufspaltung bei kurzen Perioden}

Die Abbildung 4.12 zeigt eine flächenhafte Darstellung der $\rho^{*}\left(z^{*}\right)$ Werte. Aufgrund der schlechten Datenqualität des elektrischen Feldes bei einigen Stationen existieren stellenweise Lücken in der Bedeckung.

Es sind Tiefenschnitte für drei verschiedene $z^{*}$-Bereiche dargestellt: $5-10 \mathrm{~km}, 10-20 \mathrm{~km}$, 20 - $40 \mathrm{~km}$. Dazu wurde ein Mittelwert der $\rho^{*}$-Werte, die in den jeweiligen Tiefenbereich gefallen sind, berechnet und dieser dann aufgetragen.

Die Leitfähigkeitsanomalie unter der Westeifel, die sich im magnetischen Feld so deutlich abbildet, fällt auch bei einer flächenhaften Auftragung von $\rho^{*}(y x)$ auf.

Sie ist in der ersten Schicht von 5 - $10 \mathrm{~km}$ Tiefe am deutlichsten zu erkennen. Ihre Ausprägung in der flächenhaften $\rho^{*}\left(z^{*}\right)$-Darstellung wird dann ungleichmäßig schwächer, trotzdem ist die Anomalie auch im Tiefenbereich von 20 - $40 \mathrm{~km}$ noch gut sichtbar.

In der zweiten Schicht (10 - $20 \mathrm{~km})$ erscheint eine weitere gut leitfähige Zone in beiden Polarisationen am nordwestlichen Rand des Meßgebiets ${ }^{4}$.

Die Anomalie am Südrand des Rheinischen Schiefergebirges ist in dieser Darstellung nicht zu erkennen, allerdings fehlen hier die zwei südlichen Stationen SAA und IDA. Möglicherweise ist dies auch ein weiteres Indiz dafür, daß die Anomalie erst bei der Erkundung größerer Tiefen in Erscheinung tritt.

\footnotetext{
${ }^{4}$ Es ist zu beachten, das insbesondere die dunklen Regionen an den Rändern des Meßgebiets teilweise durch den Gridding-Algorithmus des verwendeten Plot-Programms erzeugt werden.
} 

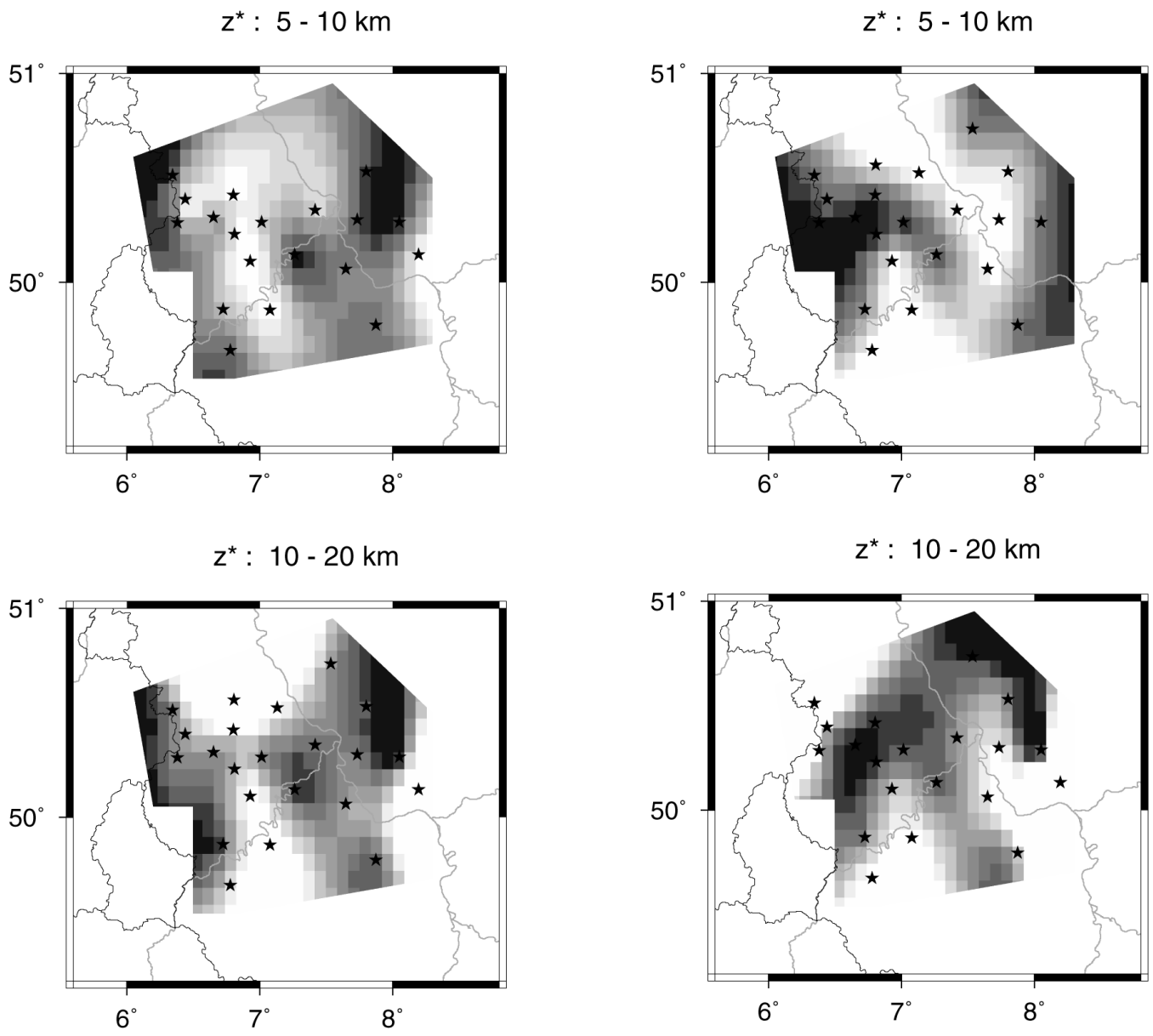

$\mathrm{z}^{\star}: 20-40 \mathrm{~km}$

$\mathrm{z}^{*}: 20-40 \mathrm{~km}$
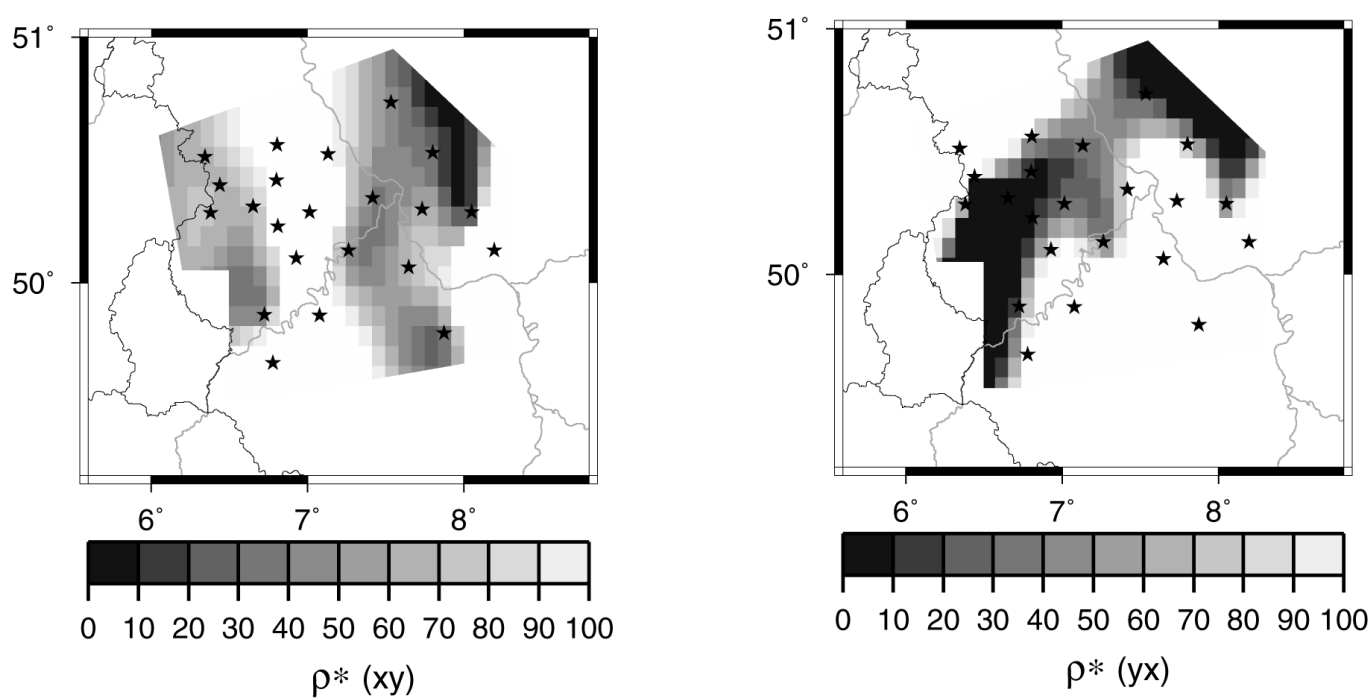

Abbildung 4.12: Flächenhafte $\rho^{*}\left(z^{*}\right)$-Darstellung. Aufgetragen ist ein Mittelwert derjenigen $\rho^{*}$-Werte, die in den dargestellten Tiefenbereich gefallen sind. Weiße Flächen kennzeichnen spezifische Widerstände $>100 \Omega \mathrm{m}$. Die Bilder der linken Spalte zeigen die $x y$-Polarisation, die der rechten Spalte die $y x$-Polarisation. 
Die Abbildung 4.4 zeigt bereits einige charakteristische Merkmale in den Frequenzgängen der Phasen, in der Abbildung 4.14 wird nun eine flächenhafte Darstellung der Phasen präsentiert (Abbildung 4.13). Die Daten wurden dabei um den Winkel $\alpha_{P}$ nach (2.22) in das Koordinatensystem gedreht, in dem eine maxinmale Phasenaufspaltung erreicht wird.

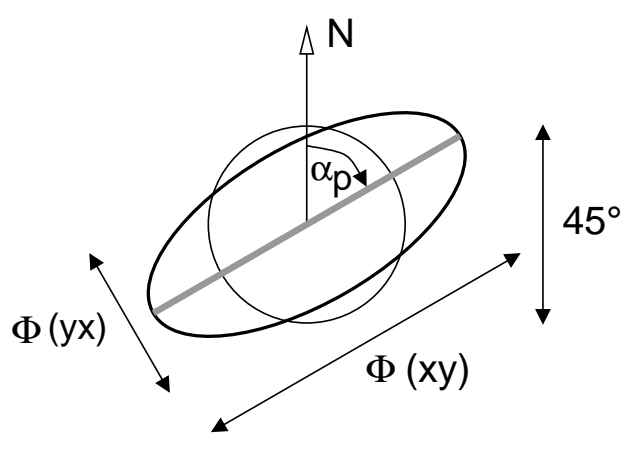

Abbildung 4.13:

Zur flächenhaften Darstellung von rotierten Phasen. Der graue Balken zeigt die rotierte $x^{\prime}$-Richtung an und stellt gleichzeitig die Größe der Phase in $x^{\prime} y^{\prime}$-Polarisation dar. Entsprechend ist die Phase der $y^{\prime} x^{\prime}$ Polarisation durch die Breite der Ellipse in der dazu senkrechten Richtung dargestellt. Als Referenz ist ein Kreis eingezeichnet, dessen Durchmesser eine Phase von $45^{\circ}$ repräsentiert.

Besonders im westlichen und nördlichen Bereich ergibt sich ein recht einheitlicher Rotationswinkel, der mit etwa $45^{\circ}$ ungefähr die Richtung des variszischen Streichens wiedergibt. An fast allen Stationen zeigt die Phase der $x^{\prime} y^{\prime}$-Polarisation die größeren Werte. Die Stationen mit der größten Phasendifferenz liegen im östlichen und nordwestlichen Teil des Meßgebiets. Die Aufspaltung liegt bei den meisten Stationen zwischen $15^{\circ}$ und $25^{\circ}$, maximal bei $34^{\circ}$ (HUR).

Für die Eindringtiefen bei der Periode 85 s erhält man etwa 10 - 20 km und somit im Vergleich zur gesamten Ausdehnung des Meßgebiets sehr kleine Werte. Das bis auf wenige Ausnahmen einheitliche Erscheinungsbild führt so zu der Vorstellung einer anisotropen Struktur in der Kruste unter dem Rheinischen Schiefergebirge (vgl. HÖNIG, 1998). 


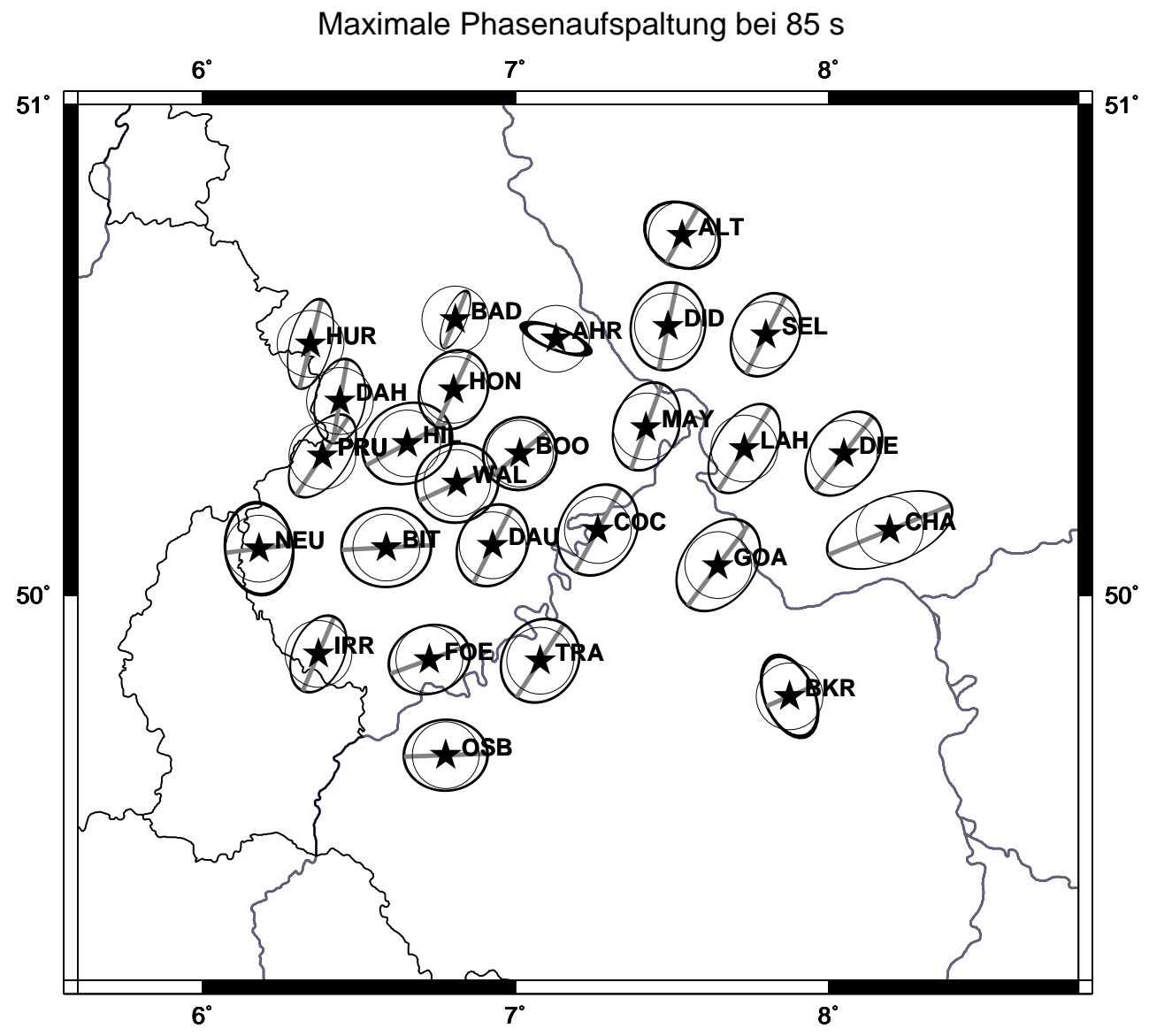

Abbildung 4.14: Darstellung der Phasen der Impedanz in dem Koordinatensystem der maximalen Aufspaltung bei einer Periode von $85 \mathrm{~s}$ (vgl. Abbildung 4.13).

\subsection{Daten zum oberen Mantel}

Betrachtet man den Periodenbereich um 1000 s und darüber, so werden Eindringtiefen erreicht, die bis in den obersten Mantel reichen.

\subsubsection{Phasenaufspaltung bei langen Perioden}

Analog zu Abbildung 4.14 sind in Abbildung 4.15 die Phasen der Impedanz bei einer Periode von $2731 \mathrm{~s}$ in dem Koordinatensystem der maximalen Aufspaltung aufgetragen. Auch hier bietet sich ein recht einheitliches Bild. Der Drehwinkel schwankt bei der Mehrzahl der Stationen um null Grad, die Richtung der größeren Phase liegt senkrecht dazu und entspricht mithin der $y^{\prime}$-Richtung. Der Median der Phasenaufspaltung liegt bei $19^{\circ}$. 
Diese einheitliche Aufspaltung der Phasen in einer bevorzugten Richtung bei langen Perioden deutet auf großräumige Strukturen in größeren Tiefen hin. Da der Drehwinkel $\alpha_{P}$ für die Periode 2731 s bei fast allen Stationen um null Grad schwankt, werden für die Betrachtung der Phasen bei langen Perioden im folgenden unrotierte Koordinaten verwendet. Die Streichrichtung verläuft dann in $y$-Richtung und $\phi_{y x}$ gehört zur E-Polarisation. Eine Richtungsabhängigkeit der Leitfähigkeit im obersten Mantel wurde bereits von HöNIG (1998) vermutet.

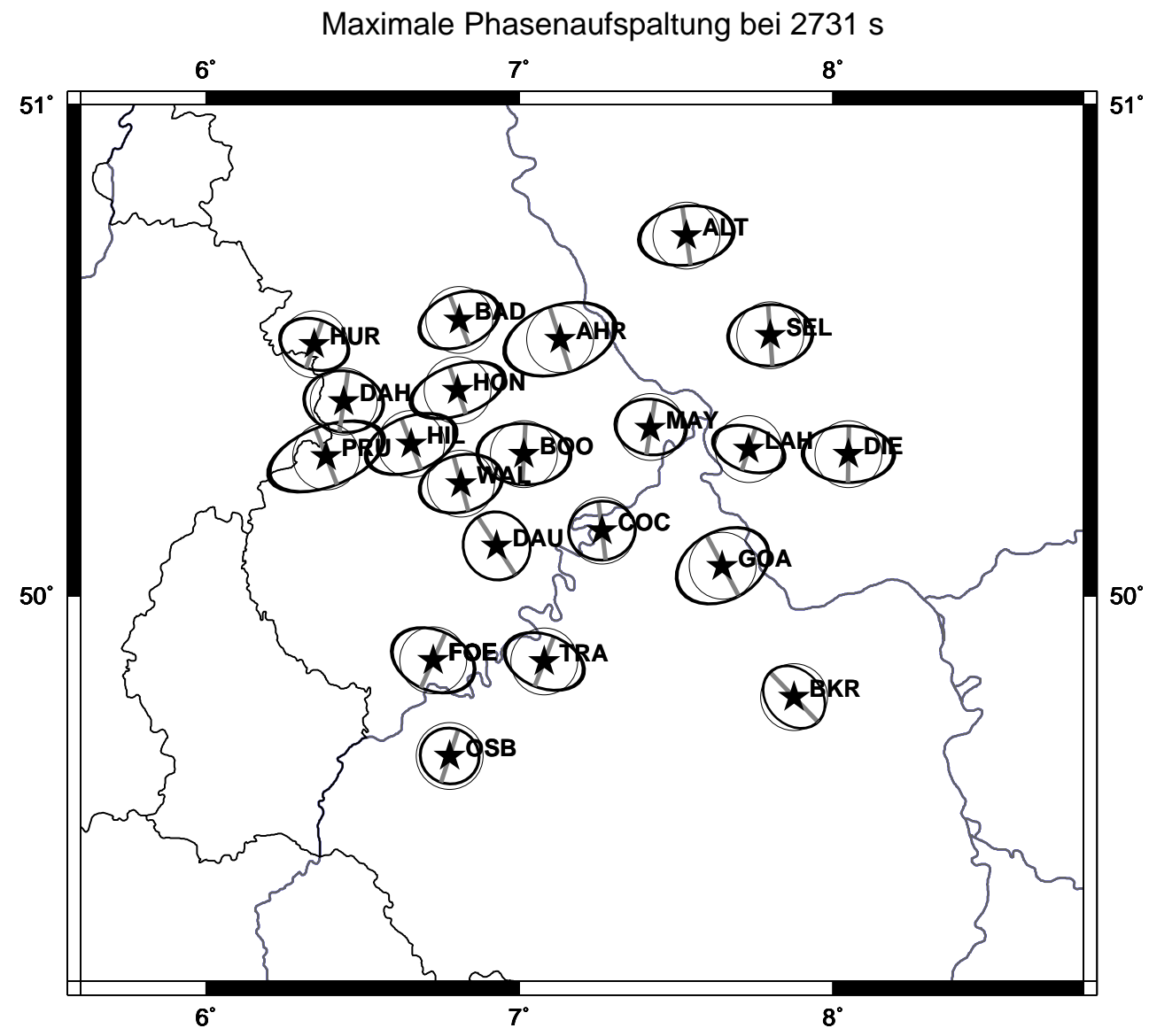

Abbildung 4.15: Darstellung der Phasen der Impedanz in dem Koordinatensystem der maximalen Aufspaltung bei einer Periode von 2731 s (vgl. Abbildung 4.13). 
Zum Nachweis, daß die Hauptinformation tatsächlich in den Nebendiagonalelementen des Impedanztensors liegt und es somit gerechtfertigt ist, nur diese zur Interpretation heranzuziehen, ist in Abbildung 4.16 das Verhältnis der Summen von Hauptdiagonal- zu Nebendiagonalelementen im Koordinatensystem der maximalen Phasenaufspaltung bei 2731 s aufgetragen. Bis auf Station GOA liegen die Verhältnisse der anderen Stationen im Mittel bei etwa 0.25 . Dies bedeutet, daß 75\% der Information, die der gesamte Impedanztensor beinhaltet, auf der Nebendiagonalen liegt.

Bei einigen Stationen im Westen weist dieses Verhältnis ungünstigere Werte auf, die jedoch zum Großteil auf eine Verzerrung durch oberflächennahe Strukturen zurückzuführen sind.

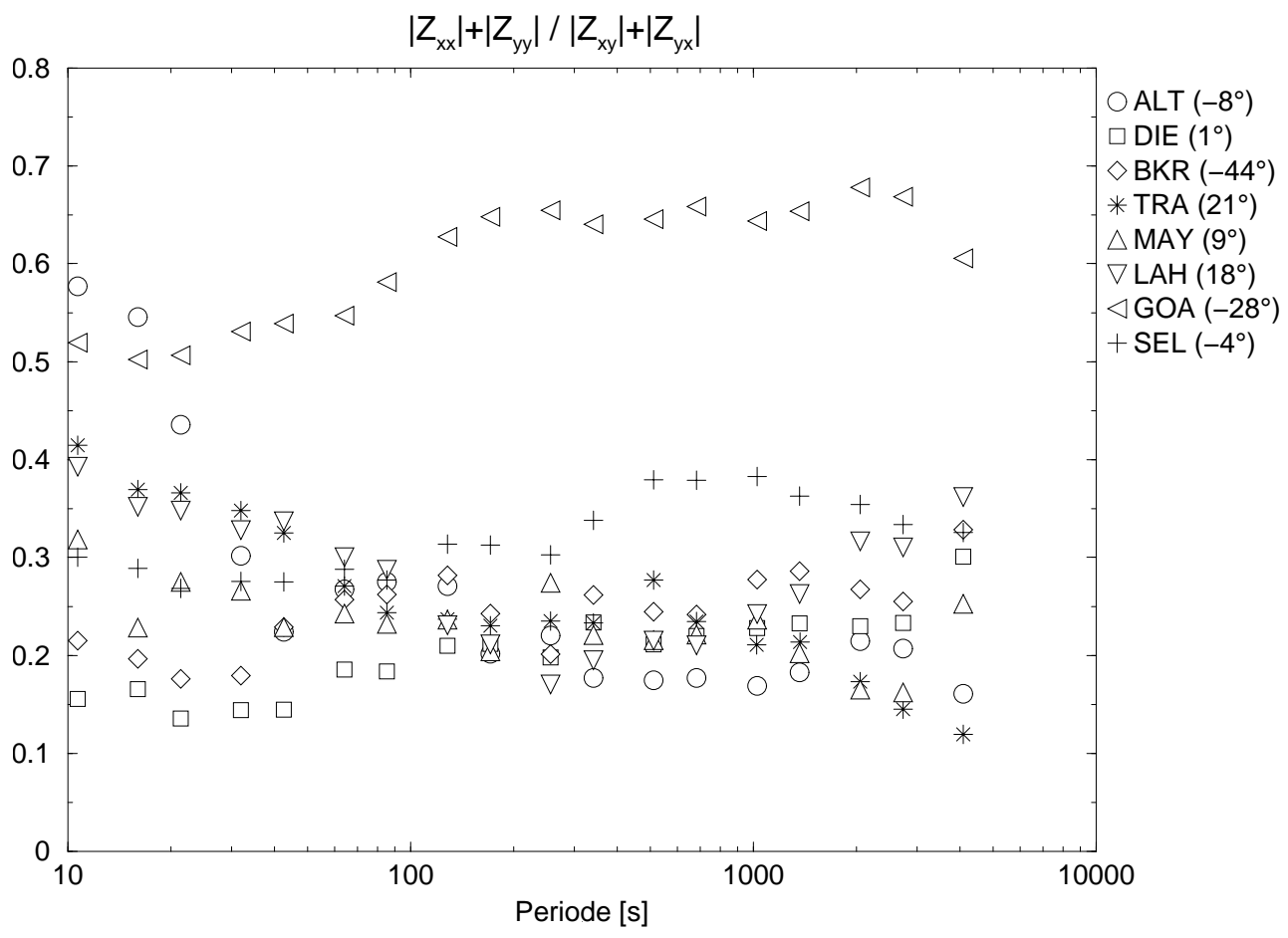

Abbildung 4.16: Das Verhältnis von Haupt- zu Nebendiagonalelementen einiger Stationen. Die Werte sind im rotierten Koordinatensystem der maximalen Phasenaufspaltung dargestellt.

In solchen Fällen ist die phasensensitive Schiefe $\eta$ aussagekräftiger (vgl. Abbildung 4.5). Wie man aus Abbildung 4.17 ersieht, weist sie im größten Teil des Meßgebiets bei der Periode $2731 \mathrm{~s}$ genügend kleine Werte unter 0.2 auf. Nur stellenweise im Westen und um die Station BOO herum kommen Werte über 0.3 vor.

Insgesamt ist also eine zweidimensionale Interpretation der Phasen der Impedanz bei langen Perioden gerechtfertigt. 


\section{$\eta(2731 \mathrm{~s})$}
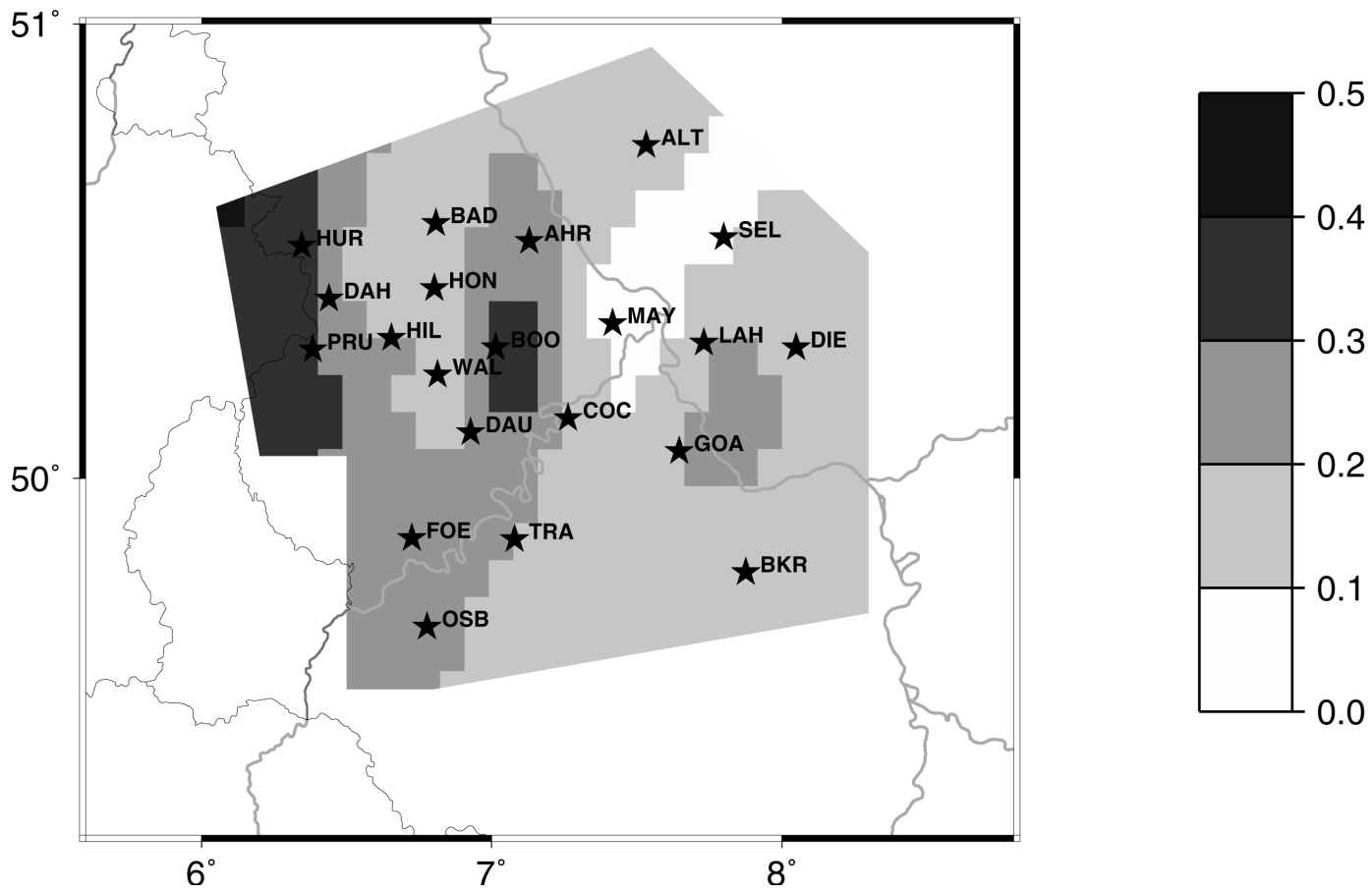

Abbildung 4.17: Flächenhafte Auftragung der phasensensitiven Schiefe $\eta$ bei einer Periode von 2731 s.

Eine flächenhafte Auftragung der Phasen beider Polarisationen in unrotierten Koordinaten findet sich in Abbildung 4.18. Während $\phi_{x y}$ nahezu im gesamten Meßgebiet bei $40^{\circ}-45^{\circ}$ liegt, sind die Werte für $\phi_{y x}$ fast überall größer als $50^{\circ}$.

Grundsätzlich sind die Werte von $\phi_{y x}$ im Norden des Meßgebiets größer als im Süden. Im Nordosten, Osten und Westen werden Phasenwerte der $y x$-Polarisation von mehr als $60^{\circ}$ erreicht. Eine Ausnahme bildet die Region der Krustenanomalie im Nordwesten. Hier liegen die Werte innerhalb eines schmalen Bereiches um DAH, HIL, WAL, HON und DAU zwischen $45^{\circ}$ und $50^{\circ}$, im südlichen Bereich (OSB) zum Teil bei nur $40^{\circ}$. 


\section{$\phi_{x y}(2731 s)$}
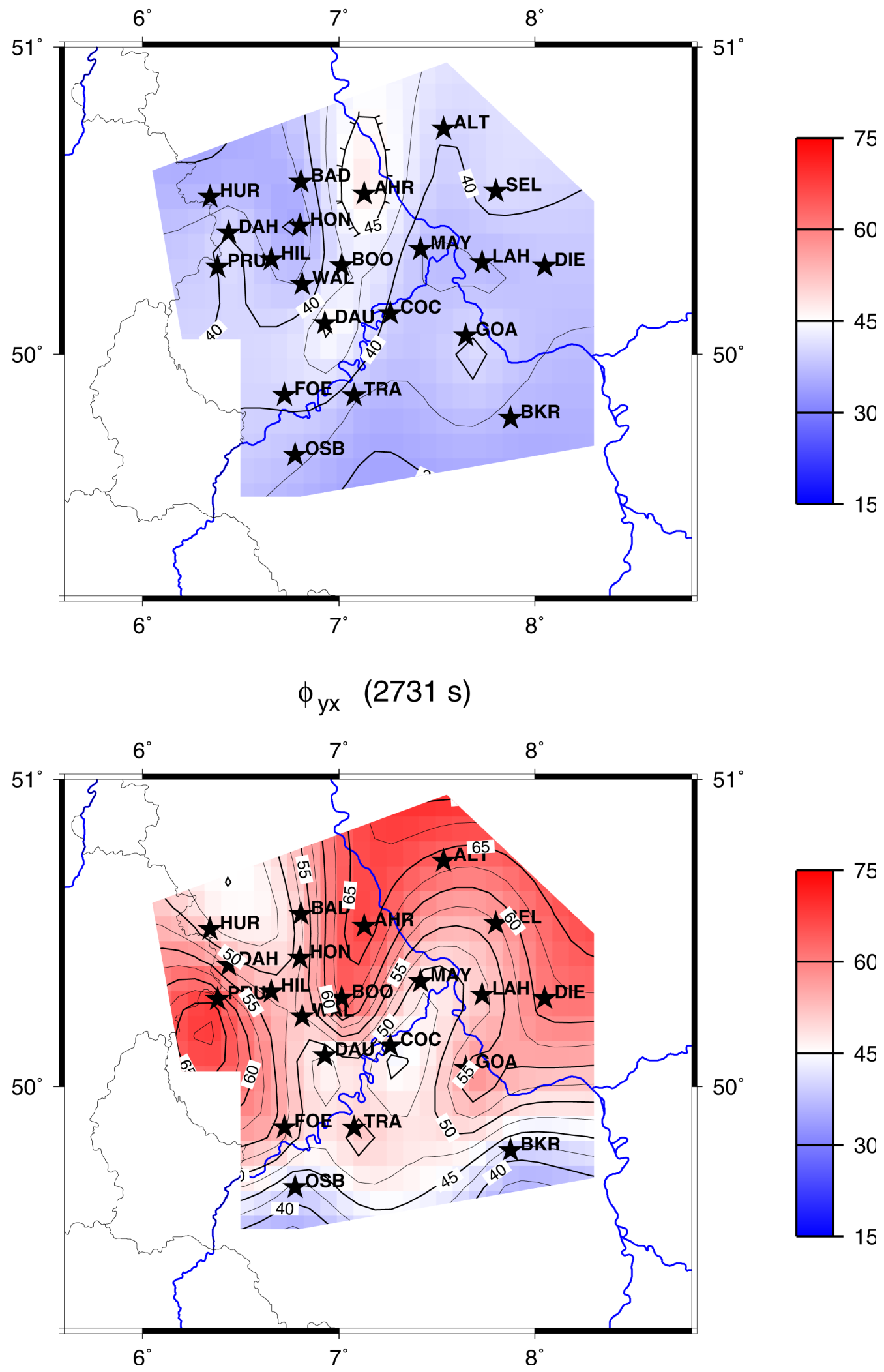

Abbildung 4.18: Darstellung von $\phi_{x y}$ und $\phi_{y x}$ für alle Stationen bei einer Frequenz von $2731 \mathrm{~s}$. 


\subsubsection{Analyse des Perturbationstensors}

Der Versuch, die gefundene Vorzugsrichtung der elektrischen Leitfähigkeit aus den Daten der erdmagnetischen Tiefensondierung mit Hilfe des in Kapitel 2.4.3 vorgestellten Verfahrens zu reproduzieren, verlief erfolglos. Die Berechnung eines Rotationswinkels $\beta_{G}$ nach Gleichung (2.34) erwies sich als nicht sinnvoll, da die gemessenen Perturbationstensoren nicht die geforderte Form (2.32) besaßen. Dies ergibt sich unmittelbar aus der Betrachtung der Schiefe $\eta_{G}$ nach Gleichung (2.33). In Abbildung 4.19 sind die Werte für drei Stationen gezeigt.

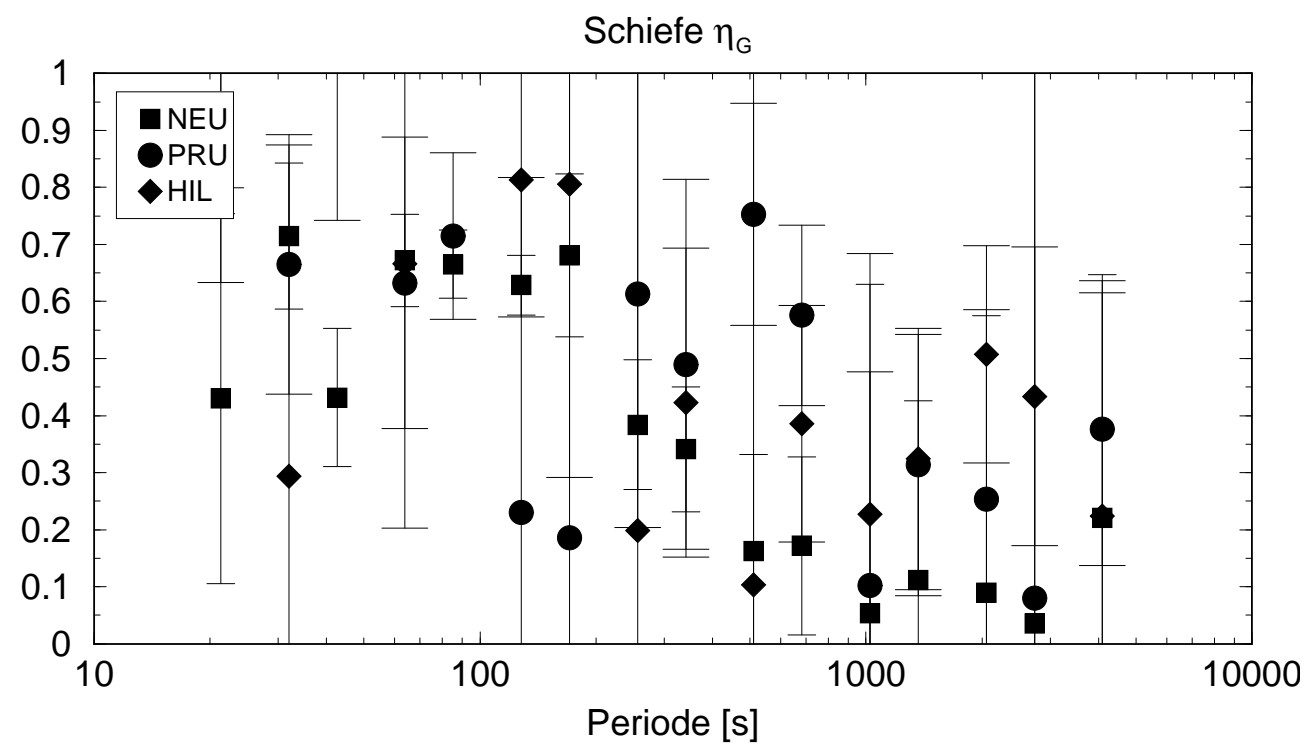

Abbildung 4.19: Frequenzgang der Schiefe $\eta_{G}$ des Perturbationstensors für drei Stationen.

Obgleich man erkennt, daß $\eta_{G}$ für lange Perioden kleiner wird, sind die Werte doch zum größten Teil über den gesamten Periodenbereich zu groß, um eine Interpretation vorzunehmen, die auf dem Modellansatz (2.31) oder (2.34) beruht. Darüberhinaus sind die Fehler relativ groß.

\subsubsection{Lokale Induktionspfeile}

Die lokalen Induktionspfeile bei 2731 s zeigen über das gesamte Meßgebiet ein einheitliches Verhalten (Abbildung 4.20). Sie weisen alle in südwestliche Richtung und zwar mit ansteigender Länge von Ost nach West. Bei den östlichen Stationen liegt die Länge etwa bei 0.1 - 0.2. Die Imaginärpfeile sind deutlich kleiner, zeigen aber zum größten Teil ebenfalls ein gleichartiges Verhalten und stehen nahezu senkrecht auf den Realpfeilen. Im Westen sind die Realpfeile mit Längen von rund 0.3 etwa doppelt so groß. Die kürzesten Pfeile findet man im Süden des Meßgebiets. Hier sind auch die Richtungen nicht in so einheitlicher Form ausgeprägt. 
Lokale Induktionspfeile bei $2731 \mathrm{~s}$

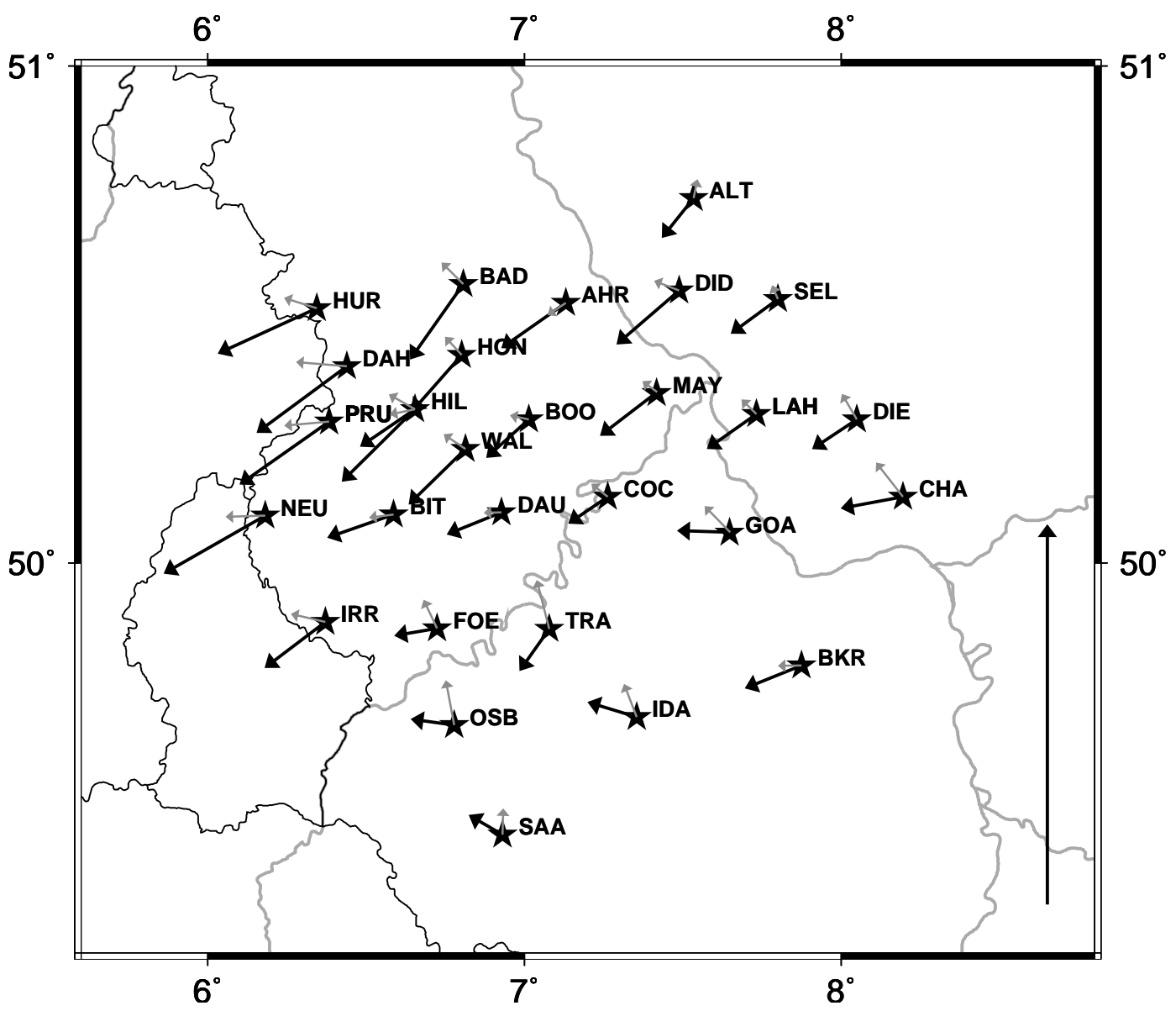

Abbildung 4.20: Darstellung der lokalen Induktionspfeile bei einer Periode von $2731 \mathrm{~s}$. Der eingezeichnete Referenzpfeil hat die Länge eins. Die Realpfeile sind schwarz dargestellt.

In Abbildung 4.21 ist abschließend das Frequenzverhalten der lokalen Induktionspfeile exemplarisch für die Stationen PRU, TRA und LAH dargestellt.

Bis zu Perioden von etwa $1000 \mathrm{~s}$ ist das Verhalten bei den drei Stationen ganz unterschiedlich. Bei mittleren Perioden 100 - $1000 \mathrm{~s}$ ist bei PRU und TRA der Einfluß der Nord-Süd bzw. West-Ost streichenden Strukturen sichtbar. Allen Stationen gemeinsam ist dann das Einschwenken der Realpfeile bei langen Perioden ab etwa $1000 \mathrm{~s}$ in südwestliche Richtung. Besonders bei TRA ist das Umklappen des Realpfeils um fast $180^{\circ}$ zwischen $683 \mathrm{~s}$ und 4096 s markant. Die Länge des Realpfeils bei PRU nimmt zu langen Perioden hin ab, während sie bei TRA zunimmt, bis die Realpfeile aller drei Stationen bei 4096 s nahezu die gleiche Länge und Richtung besitzen. 


\section{Station LAH}

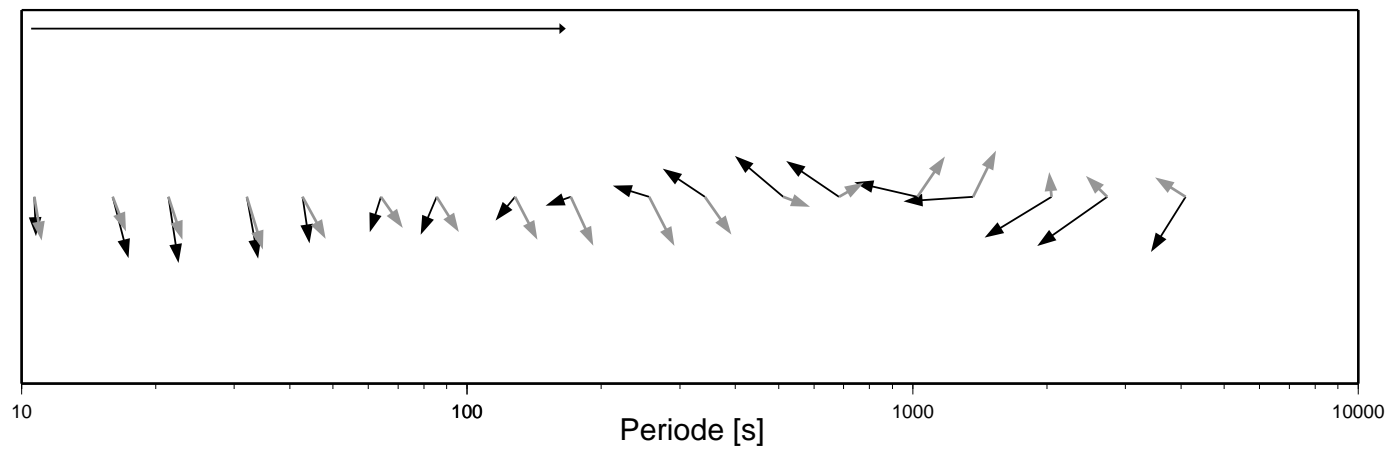

Station PRU

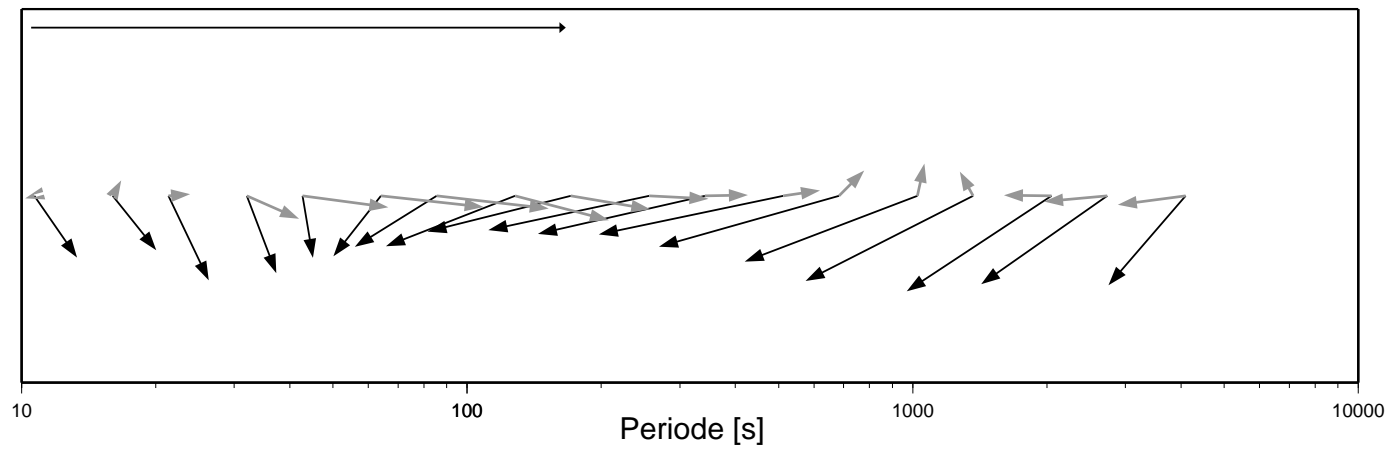

Station TRA

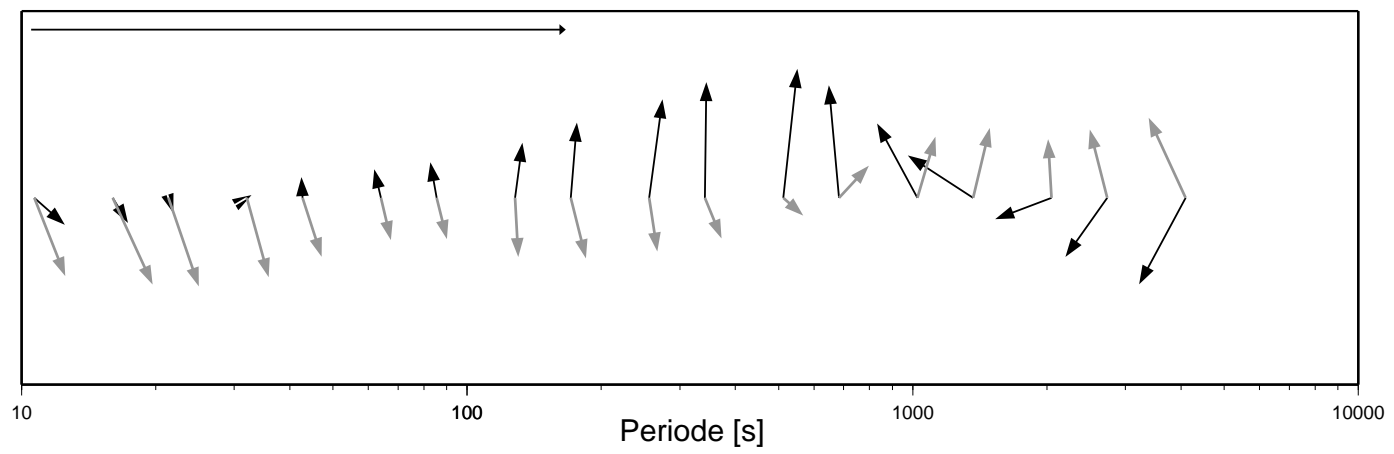

Abbildung 4.21: Frequenzverhalten der lokalen Induktionspfeile bei den Stationen PRU, TRA und LAH. Der eingezeichnete Referenzpfeil hat die Länge eins. Die Realpfeile sind schwarz dargestellt. 


\subsection{Zusammenstellung der wichtigsten Resultate}

An dieser Stelle sollen die wichtigsten Ergebnisse der Datenanalyse nochmals kurz zusammengefaßt werden:

1. Anomalie bei $256 \mathrm{~s}$ im Frequenzgang von $\operatorname{Re}\left\{d_{D}\right\}$ bei einigen Stationen in der Westeifel. Maximale Amplitude $\approx 0.4$ bei Rotation um $-17^{\circ}$. Räumliche Halbwertsbreite in West-Ostrichtung etwa $35 \mathrm{~km}$.

2. Räumlich scharf begrenzte Anomalie in den horizontalen magnetischen Übertragungsfunktionen am Südrand des Rheinischen Schiefergebirges. Maximale Amplitude von $\operatorname{Re}\left\{d_{D}\right\} \approx 0.5$ bei 683 s und Rotation um $76^{\circ}$.

3. Einheitliches Frequenzverhalten des phasensensitiven Rotationswinkels bei den meisten Stationen des Meßgebiets. Unter $200 \mathrm{~s}$ liegt $\alpha_{B}$ etwa bei $30^{\circ} \pm 15^{\circ}$, ab etwa $1000 \mathrm{~s}$ zwischen $80^{\circ}$ und $95^{\circ}$.

4. Einheitliches Frequenzverhalten der Phase der Impedanz bei den meisten Stationen des Meßgebiets.

a) Phasenwerte bei kurzen Perioden $<100 \mathrm{~s}$ in beiden Polarisationen über $45^{\circ}$. Maximale Aufspaltung der Polarisationen bei $85 \mathrm{~s}$ (bis zu 34 ) bei einem mittleren Rotationswinkel von etwa $30-40^{\circ}$.

b) Aufspaltung der Phasen bei langen Perioden $>1000 \mathrm{~s}$, dabei liegt $\phi_{x y}$ bei $40-45^{\circ}, \phi_{x y}$ erreicht zum Teil Werte $>60^{\circ}$. Maximale Aufspaltung der Polarisationen bei $2731 \mathrm{~s}$ bei einem Rotationswinkel $\approx 0^{\circ}$. Der Betrag der Phasenaufspaltung nimmt im Meßgebiet von Süden nach Norden zu.

5. Einheitliches Verhalten der lokalen Induktionspfeile bei langen Perioden über $2000 \mathrm{~s}$. Die Realpfeile weisen mit von Ost $(\approx 0.15)$ nach West $(\approx 0.3)$ zunehmender Länge in Richtung Südwesten. 


\section{Interpretation der Daten durch dreidimensionale Modelle}

Die durchgeführten Modellsimulationen wurden als dreidimensionale Vorwärtsrechnungen realisiert. Dabei wurde der Finite-Differenzen-Algorithmus nach MACKIE et al. verwendet (MACKIE et al., 1993; MACKIE \& MAdDEN, 1993; MACKIE et al., 1994). Die Berechnung synthetischer Übertragungsfunktionen aus den Resultaten der Modellrechnungen, die mit den gemessenen vergleichbar sind, wird bei KURAS (1998) beschrieben.

\subsection{Zur Modellentwicklung}

Die Fülle des vorliegenden Datenmaterials macht es notwendig, sich auf einige besondere Merkmale zu beschränken, die durch das Modell erklärt werden sollen. Bestimmte Charakteristika der Daten lassen im Vorfeld bereits Schlüsse auf möglicherweise zugrunde liegende Leitfähigkeitsanomalien zu (vgl. Kapitel 2 und 4). So entsteht eine erste Vorstellung der Leitfähigkeitsverteilung unter dem Meßgebiet, die allerdings zum größten Teil auf relative Widerstandskontraste und Umrisse möglicher anomaler Bereiche beschränkt ist. Bevor diese Erkenntnisse in die Entwicklung eines konkreten dreidimensionalen Modells umgesetzt werden, ist es zur Skalierung notwendig, ein Hintergrundmodell zu entwerfen, in das die anomalen Bereiche eingebettet werden. Dieses besteht in der Regel aus einem geschichten Halbraum mit definierten Widerstandswerten.

Die Optimierung des Modells geschieht dann durch sukzessive Veränderung verschiedener Parameter. Im allgemeinen sind dies spezifische Widerstandswerte und die räumliche Ausdehnung anomaler Bereiche. Anschließend wird das neue Modellergebnis mit den Felddaten verglichen und die Modellstruktur daraufhin eventuell verändert um die Anpassung weiter zu verbessern.

In der Praxis ist es nicht möglich, alle vorhandenen Parameter gleichermaßen zu variieren, so daß es sinnvoll ist, für einige von ihnen im Vorfeld bestimmte Werte festzulegen, die nicht oder nur sehr geringfügig verändert werden. Diese können sich zum Beispiel aus ein- oder zweidimensionalen Inversionsrechnungen ergeben. Möglicherweise liegen bereits anderweitige geophysikalische oder geologische Erkenntnisse vor, die direkt umgesetzt werden können oder Grenzen der zu variierenden Größen fordern.

Im Laufe der Modellrechnungen werden sich einige (wenige) Parameter herauskristallisieren, auf deren Variation die anzupassenden Datenmerkmale besonders sensibel rea- 
gieren. Liegt das endgültige Grundmodell vor, das die geforderten Ansprüche hinreichend befriedigt, ist es sinnvoll, diese Parameter nochmals systematisch zu variieren. Dadurch erhält man einen Überblick über den möglichen Variationsbereich der postulierten Leitfähigkeitsverteilung. Denn das vorgeschlagene Modell ist natürlich nicht eindeutig und alle Parameter können innerhalb gewisser Grenzen schwanken.

In der folgenden Grafik 5.1 sind die beschriebenen Schritte nochmals tabellarisch zusammengefaßt.

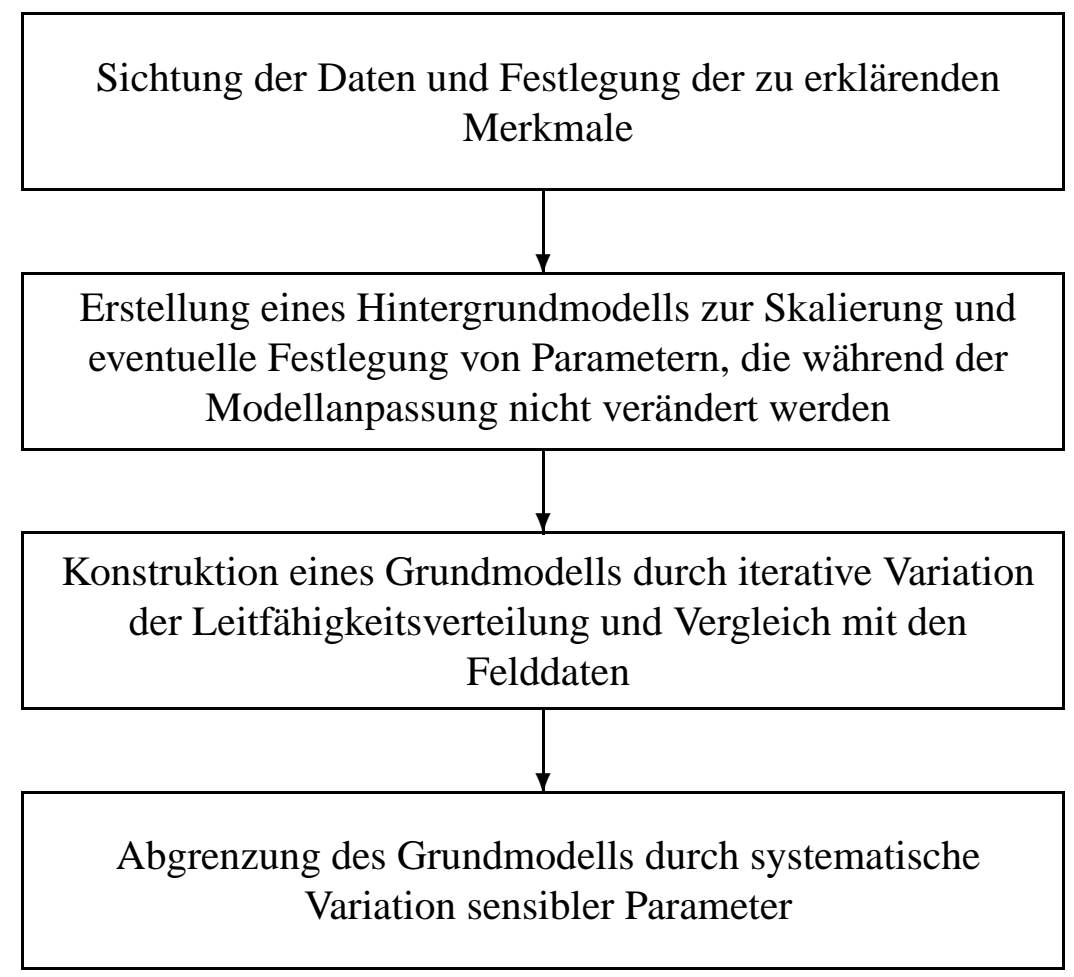

Abbildung 5.1: Strukturierte Entwickung eines Modells der Leitfähigkeitsverteilung mit Hilfe von Vorwärtsrechnungen.

\subsection{Vorbereitungen}

\subsubsection{Sichtung der Daten}

Bei der Analyse der Daten ergaben sich einige besonders charakteristische Merkmale, die in Kapitel 4.4 zusammengestellt sind. Das zu entwickelnde Modell der Leitfähigkeitsverteilung unter dem Rheinischen Schiefergebirge sollte mehreren Ansprüchen genügen und folgende Merkmale in integrierter Form erklären können:

- Anomalie in der Westeifel: räumliche Ausdehnung und Frequenzverhalten der magnetischen Übertragungsfunktionen 
- Einheitliches Frequenzverhalten des phasensensitiven Rotationswinkels über dem Array

- Charakteristischer Frequenzgang und räumliches Verhalten der Phase der Impedanz

- Verhalten der lokalen Induktionspfeile

Die Anomalie im Süden des Rheinischen Schiefergebirges wird dagegen nicht berücksichtigt. Wie in Kapitel 4 eingehend besprochen wurde, zeigen die Stationen südlich der Mosel zum Teil ein deutlich abweichendes Verhalten. Ihre Hinzunahme würde eine starke Verkomplizierung des Modells bedeuten, da in diesem Fall ein einheitliches Hintergrundmodell nicht angebracht wäre. Zudem wurde bereits gezeigt, daß sich die Effekte beider Anomalien in unterschiedlichen Polarisationen abbilden und so recht gut getrennt betrachtet werden können. Erste Ansätze zur Modellierung der Anomalie im Bereich der Hunsrück-Südrand-Störung findet man bei KURAS (1998).

\subsubsection{Das Hintergrundmodell}

Zur Erstellung eines geschichteten Hintergrundmodells wurde eine 1D-Inversion der mittleren Impedanz $Z_{\text {eff }}^{I}=\left(Z_{x y}-Z_{y x}\right) / 2$ (Gleichung 2.11) durchgeführt. Dieser Parameter ist rotationsinvariant, weshalb er sich zur Erstellung eines eindimensionalen Modells anbietet.

Des weiteren wird die Berdichevsky-Invariante durch kleinere Abweichungen vom 1DFall weniger stark beeinflußt als $Z_{x y}$ oder $Z_{y x}$. Durch die Mittelung über beide Polarisationen kann unter Umständen der störende Einfluß einer Verzerrung des elektrischen Feldes verringert werden. Wenn man davon ausgeht, daß durch eine Verzerrung beide Polarisationen des scheinbaren spezifischen Widerstandes annähernd symmetrisch zur 1D-Kurve (ohne lokalen Störer) aufgespalten werden, liegt der Mittelwert der Impedanz nahe der Kurve des geschichteten Halbraums (vgl. Abbildung 1.8). Nur in Grenzfällen ist es denkbar, daß beide Polarisationen nach der Aufspaltung über oder unter der eindimensionalen Kurve liegen. Falls allerdings tatsächlich eine zweidimensionale Struktur vorliegt, ergibt die Mittelung einen Wert für die Impedanz, der ohne Aussagekraft ist. Dann müßte sich diese Struktur allerdings auch in weiteren Größen widerspiegeln und ohnehin als zweioder dreidimensionale Leitfähigkeitsanomalie in das Modell eingebaut werden. In der folgenden Abbildung 5.2 sind die Ergebnisse für eine 4-Schicht Inversion von $Z_{\text {eff }}^{I}$ für 9 Stationen gezeigt, die über das gesamte Meßgebiet verteilt sind.

Die Inversion wurde mit dem $\Psi$-Algorithmus durchgeführt (SCHMUCKER, 1974). Eine Erhöhung der Schichtenanzahl $>4$ führte zu keinem signifikant kleineren Anpassungsfehler.

Zunächst fällt auf, daß sich die Ergebnisse der südlichen Stationen auch hier von denen der übrigen Standorte deutlich unterscheiden. Die Stationen nördlich der Mosel zeigen einen guten Leiter in der mittleren bis unteren Kruste $(15-25 \mathrm{~km})$. Auch bei OSB und GOA wird ein guter Leiter sichtbar, allerdings erst in Tiefen zwischen 30 und 40 
1D-Inversion der Berdichevsky-Invarianten

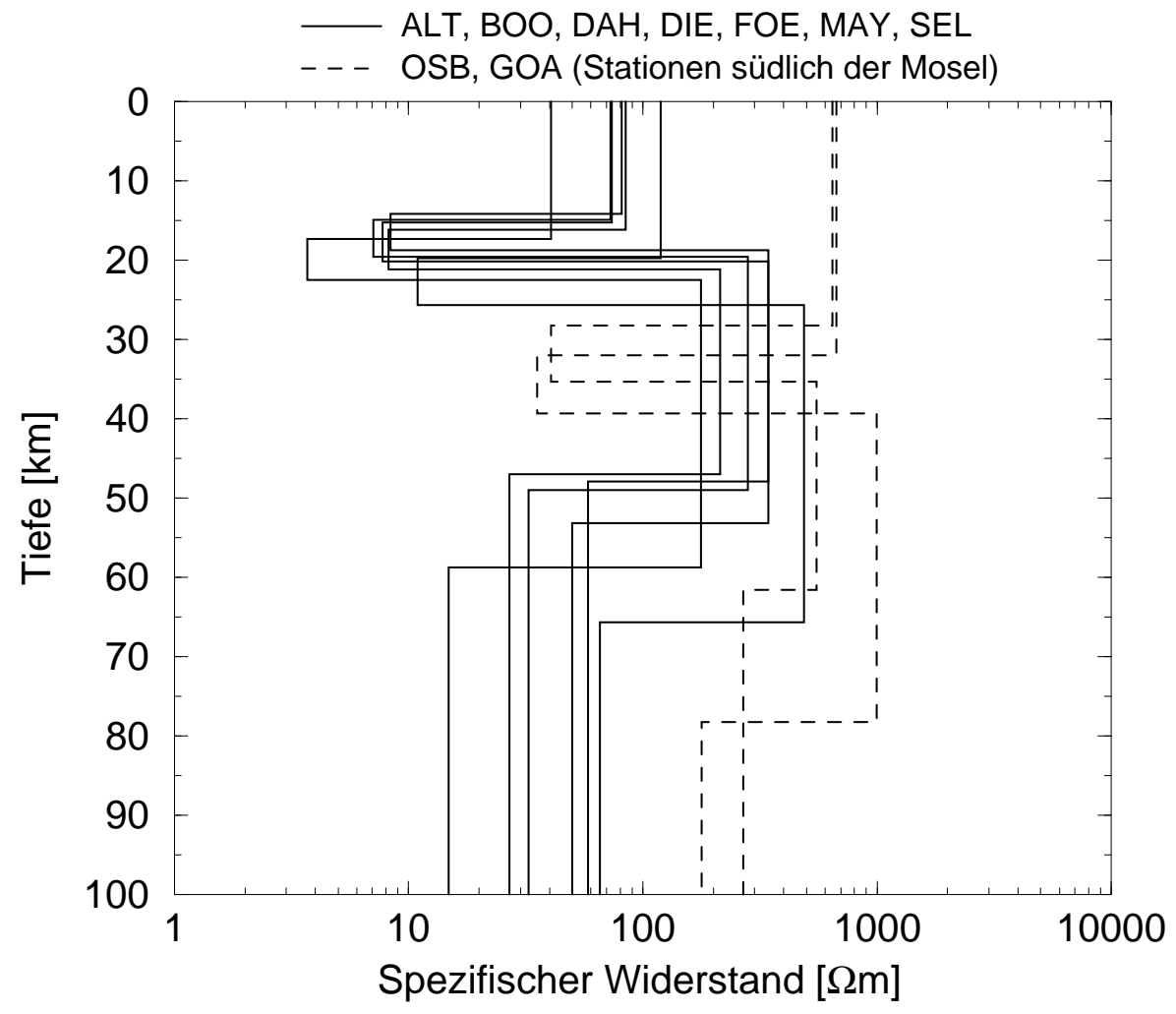

Abbildung 5.2: Ergebnisse einer 4-Schicht Inversion der effektiven Impedanz $Z_{\mathrm{eff}}^{I}=\left(Z_{x y}-\right.$ $\left.Z_{y x}\right) / 2$ für 9 Stationen.

km. Darüberhinaus weisen die spezifischen Widerstände in der oberen Kruste große Unterschiede auf. Die gemittelten spezifischen Widerstände bei den Stationen nördlich der Mosel liegen bei 40 - $110 \Omega \mathrm{m}$, im Vergleich zu 600 - $700 \Omega \mathrm{m}$ an den Stationen OSB und GOA.

\subsubsection{Zur Modellierung einer anisotropen Schicht}

Der verwendete 3D-Code nach MACKIE et al. gestattet nicht den Einbau einer intrinsischen Anisotropie, so daß auf das Modell der makroskopischen Anisotropie zurückgegriffen wurde. GroOM \& BAILEY (1989b) zeigen, daß eine Folge von schmalen, hochgestellten Platten (sogenannte Dykes) mit jeweils unterschiedlicher Leitfähigkeit den gewünschten Effekt auf magetotellurische Übertragungsfunktionen erzielen. In der Praxis werden diese Lamellen jeweils eine einheitliche Länge $L$ und Höhe $h$ haben. Der Aufbau einer solchen Schicht ist in Abbildung 5.3 skizziert.

Interessant ist der spezifische Widerstand, den die anisotrope Schicht jeweils in Richtung $\left(\rho_{\|}\right)$und senkrecht $\left(\rho_{\perp}\right)$ zu den Lamellen besitzt. Betrachtet man die Anordnung als eine 


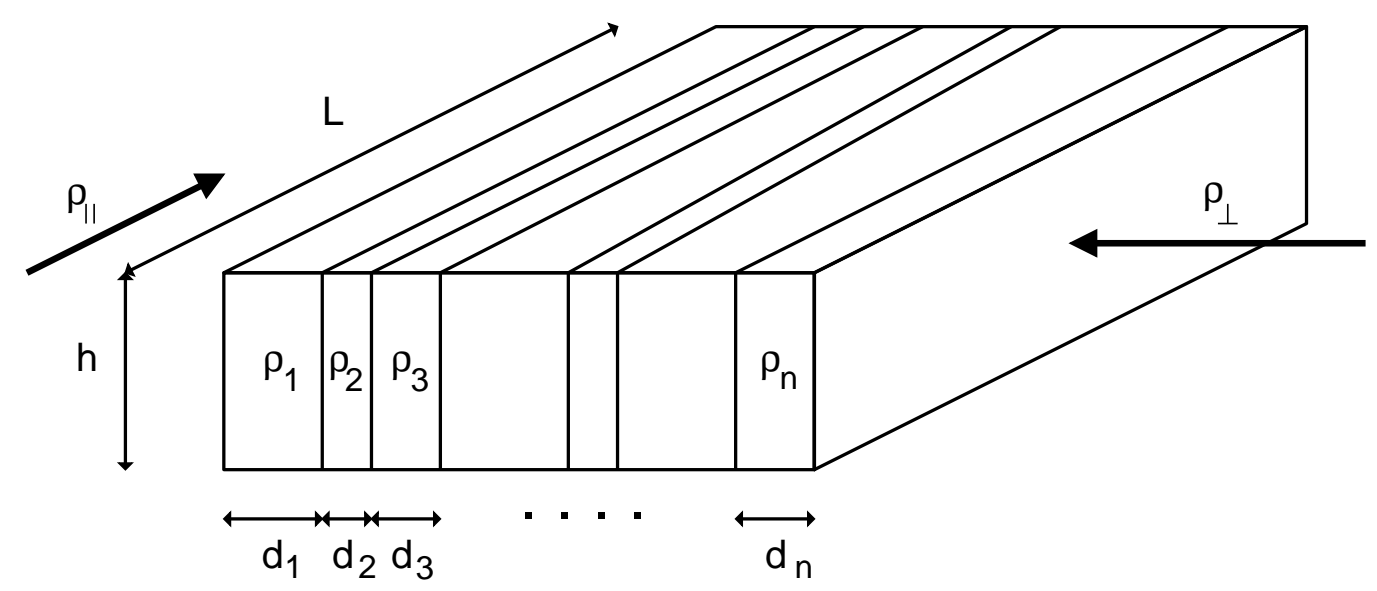

Abbildung 5.3: Realisierung einer anisotropen Schicht durch das Lamellenmodell.

Reihen- bzw. Parallelschaltung von Ohmschen Widerständen $R_{i}=\rho_{i} l / A$, so erhält man $\rho_{\perp} \sum d_{i}=\sum \rho_{i} d_{i}$ und $\sum d_{i} / \rho_{\|}=\sum\left(d_{i} / \rho_{i}\right)$. Eine weitere Vereinfachung wird erreicht, wenn insgesamt nur eine Folge von zwei Lamellen mit unterschiedlichen Widerständen und Dicken verwendet wird. Man erhält dann:

$$
\rho_{\|}=\frac{\rho_{1} \rho_{2}\left(d_{1}+d_{2}\right)}{\rho_{1} d_{2}+\rho_{2} d_{1}} \quad \text { und } \quad \rho_{\perp}=\frac{\rho_{1} d_{1}+\rho_{2} d_{2}}{d_{1}+d_{2}}
$$

Um ein gegebenes Paar $\rho_{\|}, \rho_{\perp}$ zu realisieren, sind beliebig viele Kombinationen der 4 Parameter $\rho_{1}, d_{1}, \rho_{2}, d_{2}$ denkbar. Gibt man zum Beispiel die Dicke und den Widerstand einer Lamelle vor, so lassen sich die zugehörigen Werte der anderen berechnen.

Die Ausdehnung der Lamellenstruktur sowohl in $x$-Richtung als auch in $y$-Richtung kann im Modell nicht unendlich fortgesetzt werden. Der jeweils anschließende Randbereich ist eindimensional. EISEL (1995) schlägt vor, für die angrenzenden Gebiete die jeweiligen Hauptwiderstände $\rho_{\|}$und $\rho_{\perp}$ zu verwenden. Um Randeffekte aufgrund des unstetigen Verhaltens des elektrischen Feldes an den vertikalen Leitfähigkeitskontrasten der Lamellen im Fall der B-Polarisation zu glätten, ist eine lateral homogene Deckschicht mit hinreichend großer Mächtigkeit ( $>$ Breite der gutleitenden Lamellen) notwendig (EISEL \& HAAK, 1999). In der vorliegenden Arbeit wird ein Modelltyp vorgeschlagen, der versetzte Lamellen verwendet (Abbildung 5.4).

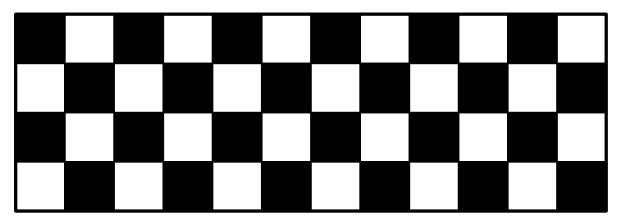

$\rho_{1} \amalg \rho_{2}$
Abbildung 5.4:

Querschnitt durch eine anisotrope Schicht, die mit dem Modell der versetzten Lamellen realisiert wurde. 
Aus Gleichung (5.1) geht bereits hervor, daß sich mit Lamellen gleicher Breite $d_{1} / d_{2}=1$ jedes beliebige Paar $\rho_{\|}, \rho_{\perp}$ konstruieren läßt. Die Lamellen werden hier vertikal nochmals in mehrere Schichten unterteilt, die dann jeweils um eine Lamellenbreite versetzt werden. Die folgende Abbildung 5.5 zeigt zwei einfache Modelle, die beide eine anisotrope Schicht mit $\rho_{\|}=1.9 \Omega \mathrm{m}$ und $\rho_{\perp}=10.5 \Omega \mathrm{m}$ simulieren. Abbildung 5.6 zeigt die zugehörigen Widerstands- und Phasenkurven, sowie zum Vergleich das Ergebnis eines Modells, in welchem die anisotrope Schicht aus Modell A vertikal in 6 Schichten unterteilt wurde, die gegeneinander versetzt angeordnet wurden.

Modell A

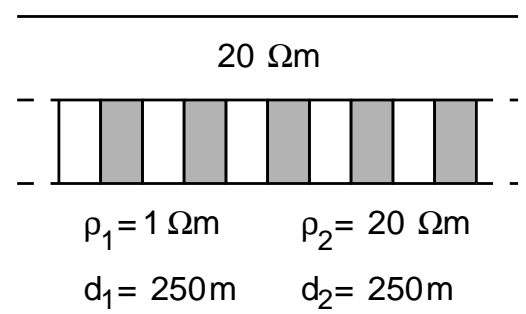

Modell B

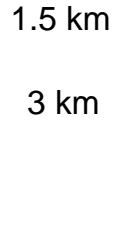

$20 \Omega m$

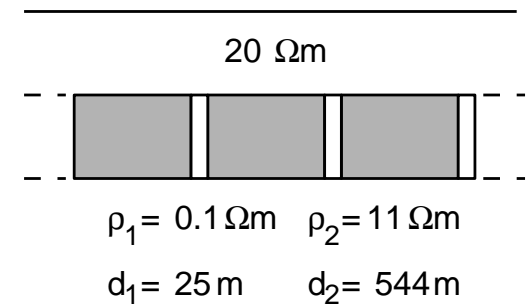

Abbildung 5.5: Zwei Modelle, die jeweils eine anisotrope Schicht mit $\rho_{\|}=1.9 \Omega \mathrm{m}$ und $\rho_{\perp}=10.5 \Omega \mathrm{m}$ simulieren. Der abschließende Halbraum besitzt den spezifischen Widerstand $20 \Omega \mathrm{m}$.

Man erkennt, daß die Kurven aller Modelle bis etwa 70 s deckungsgleich übereinander liegen.

Beide Polarisationen des scheinbaren spezifischen Widerstandes spalten bei mittleren Perioden 10 - $100 \mathrm{~s}$ maximal auf und laufen dann wieder zusammen, erreichen aber nicht ganz den Wert des abschließenden Halbraums. Die Kurven der $y x$-Polarisation der verschiedenen Modelltypen weichen in diesem Periodenbereich geringfügig voneinander ab. Bei dem Modell mit versetzten Lamellen konvergieren die beiden Polarisationen bei langen Perioden am besten gegen den Widerstand des abschließenden Halbraums.

Die Phasenkurven der $y x$-Polarisation zeigen den typischen Effekt einer gut leitenden Zwischenschicht in besonders ausgeprägter Form auf. Sie liegen bei kurzen Perioden über $45^{\circ}$ und wechseln dann im Periodenbereich, in dem der zugehörige $\rho_{a}$-Wert sein Minimum erreicht, unter $45^{\circ}$.

Auch im Randbereich der anisotropen Schicht zeigt das Modell der versetzten Lamellen geringfügige Vorteile. In Abbildung 5.7 sind zwei Querprofile von $\rho_{a}(x y)$ über dem Modell A berechnet worden, bei denen die anisotrope Schicht mit versetzten und unversetzten Lamellen realisiert wurde. Die $\rho_{a}$-Kurven, die über dem Modell mit versetzten Lamellen bestimmt wurden, zeigen im Randbereich ein glatteres Verhalten. Die Schwankung der anderen $\rho_{a}$-Kurve beträgt etwa $0.5 \Omega \mathrm{m}$, bei einer geringeren Mächtigkeit der Deckschicht verstärkt sich dieser Effekt noch. 

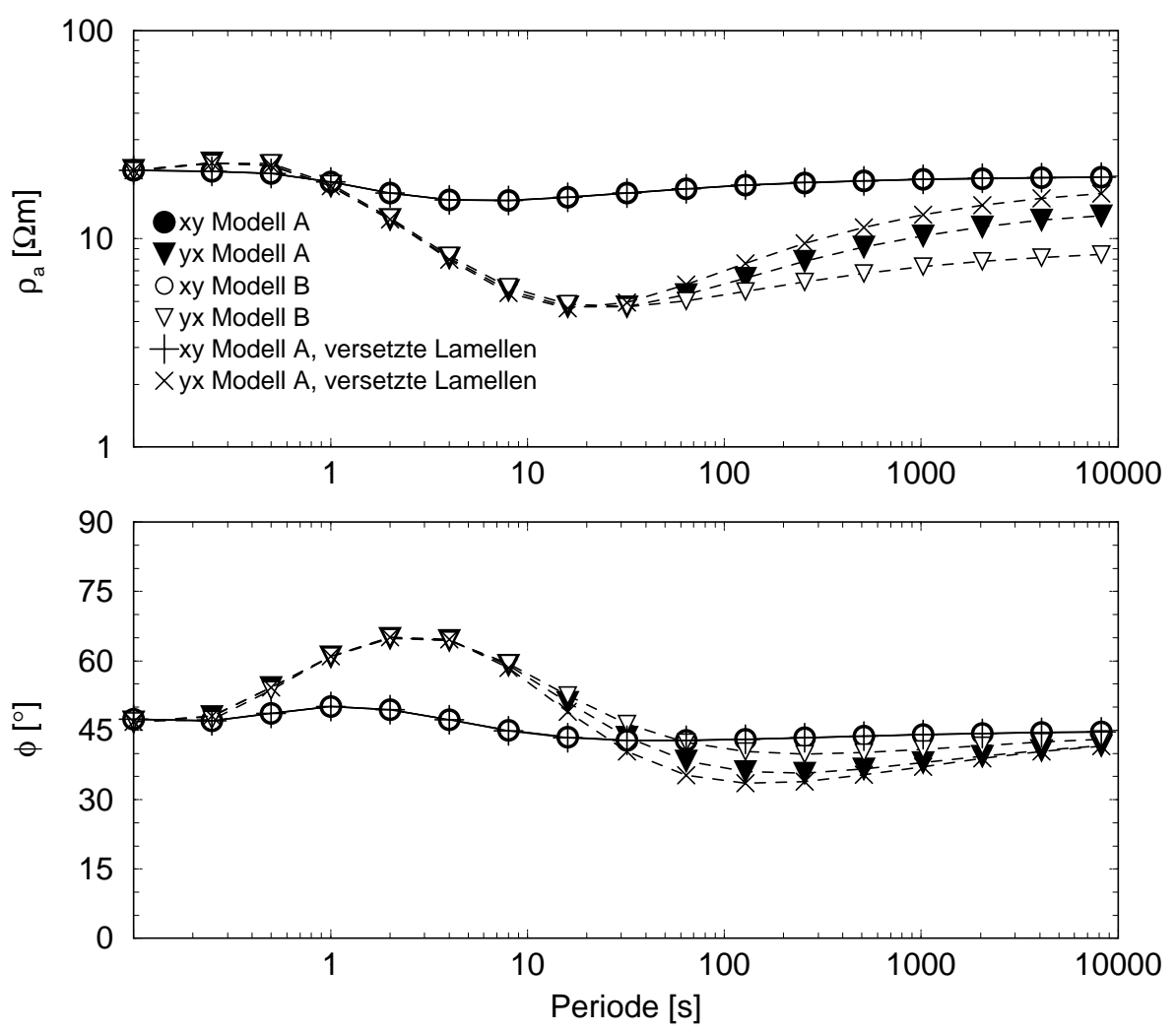

Abbildung 5.6: $\rho_{a^{-}}$und Phasenkurven der Modelle A und B aus Abbildung 5.5.

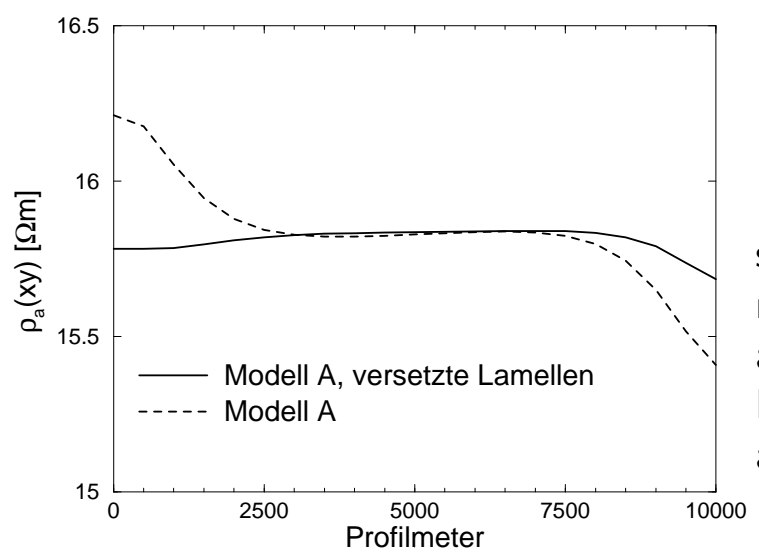

Abbildung 5.7:

Scheinbarer spezifischer Widerstand der B-Polarisation über einem Querprofil von Modell A. Die anisotrope Schicht besteht aus 40 Lamellen, die versetzt/unversetzt angeordnet werden. 


\subsubsection{Diskretisierung des 3D-Modellkernes}

Der gesamte Modellaufbau wird beim MACKIE-Code in mehrere Bereiche aufgeteilt. Die eigentliche dreidimensionale Struktur wird innerhalb eines 3D-Modellkernes realisiert, welcher in regionale 2D-Modelle eingebettet ist, die zur Berechnung der Randwerte dienen. In vertikaler Richtung wird der 3D-Kern durch einen geschichteten Halbraum abgeschlossen.

Zunächst muß die Dimensionierung des dreidimensionalen Modellkernes festgelegt werden. In Abbildung 5.8 ist die horizontale und vertikale Diskretisierung zusammen mit den Meßpunkten dargestellt.
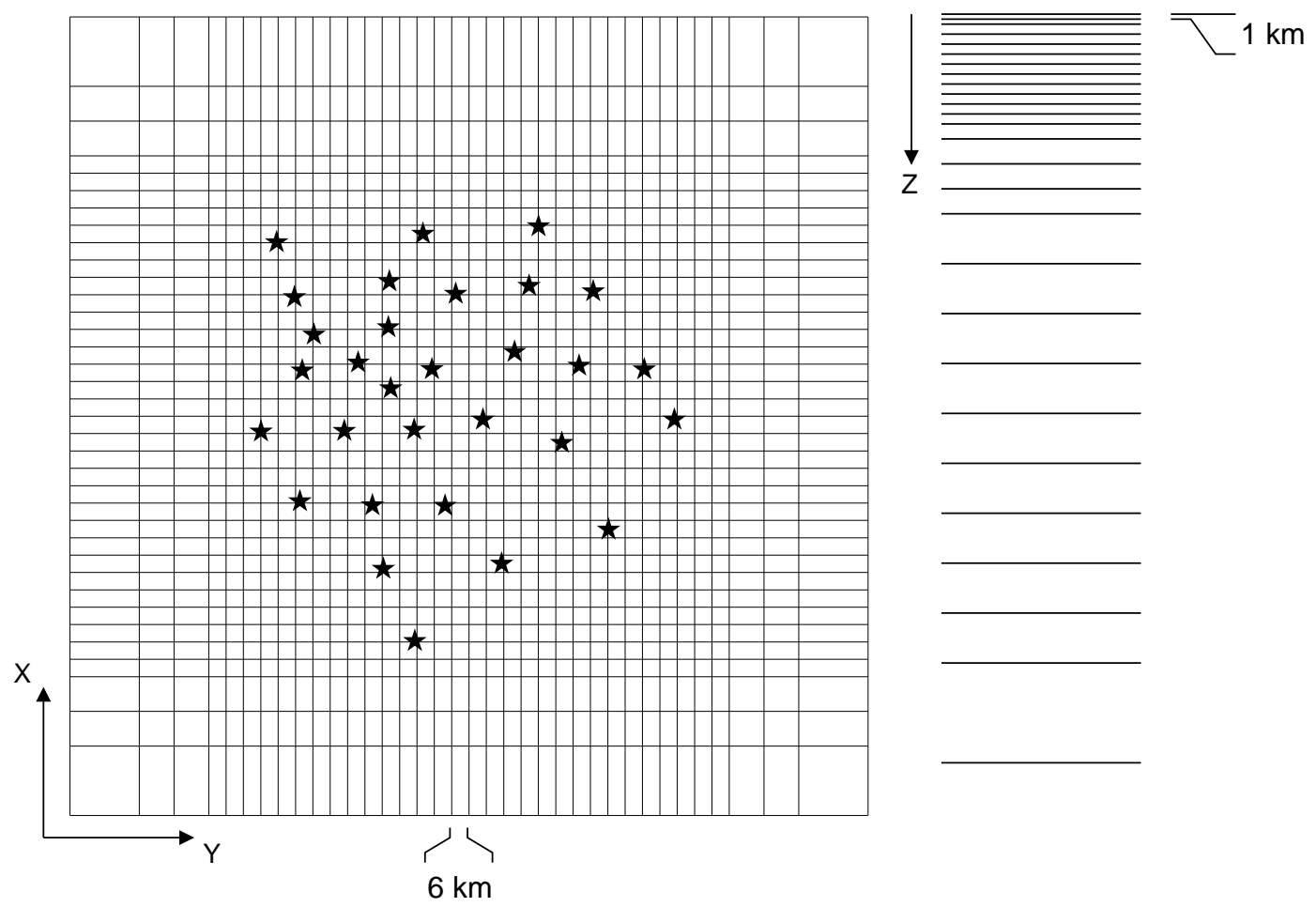

Abbildung 5.8: Horizontale und vertikale Diskretisierung des 3D-Modellkernes.

Insgesamt werden $36 \times 36 \times 27$ Modellzellen verwendet. Der Modellkern überdeckt eine Fläche von $276 \mathrm{~km} \times 276 \mathrm{~km}$, die Zellgröße im inneren Bereich beträgt $6 \mathrm{~km} \times 6 \mathrm{~km}$, wobei sich die Größe in den äußeren Bereichen mit zunehmendem Abstand vom inneren Bereich verdoppelt. Dies gilt auch für die Zellen der 2D-Randmodelle.

In vertikaler Richtung erstreckt sich der Modellkern bis in eine Tiefe von $190 \mathrm{~km}$. Die obersten Schichten besitzen eine Mächtigkeit von $1 \mathrm{~km}$ und werden dann mit zunehmender Tiefe dicker. 


\subsection{Ein Modell der Leitfähigkeitsverteilung unter der Eifel}

\section{Das Grundmodell}

In diesem Abschnitt wird ein Modell der Leitfähigkeitsverteilung unter dem Rheinischen Schiefergebirge präsentiert. Um es von späteren Modellrechnungen abzugrenzen, wird es als das Grundmodell bezeichnet (Abbildung 5.9).

Die wichtigsten Überlegungen, die im einzelnen zu seiner Entwicklung führten, werden in der folgenden Übersicht skizziert. Beginnend mit der oberen Kruste wird die Wahl der einzelnen Parameter begründet.

- Ausgehend von der 1D-Inversion der Berdichevsky-Invarianten (Abbildung 5.2) wird ein Widerstandswert von $80 \Omega$ m für die obere Kruste angenommen, der im Zuge der Modellanpassung nicht variiert wird. Frühere Untersuchungen bestätigen diese Größenordnung des spezifischen Widerstandes der Kruste im Rheinischen Schiefergebirge, insbesondere im Gebiet nördlich der Hunsrück-Südrand-Störung: JÖDICKE (1983) und später OETTINGER (1994) ermittelten im Bereich der Osteifel unter Berücksichtigung einer möglichen statischen Verzerrung spezifische Widerstände der oberen Kruste im Bereich von $80-115 \Omega \mathrm{m}$. An einer Station südlich der Mosel (etwa zwischen COC und GOA) fand BAHR (1985) einen spezifischen Widerstand von ca. $150 \Omega$. Für das Gebiet der Westeifel liegen noch keine Vergleichsdaten vor.

\section{- Inhomogene Schicht 1: Leitfähigkeitsanomalie in der nordwestlichen Eifel}

Durch Anpassung der Frequenzgänge der magnetischen Übertragungsfunktionen $d_{D}$ und $z_{D}$ der Stationen HON und HIL (Abbildung 4.7) wird die Tiefenlage (6 km) und integrierte Leitfähigkeit (4000 S) der nordwestlichen Leitfähigkeitsanomalie bestimmt.

Die räumliche Ausdehnung in den horizontalen Richtungen ergibt sich dabei aus der flächenhaften Auftragung der horizontalen magnetischen Übertragungsfunktionen (Abbildung 4.9). Die Anomalie besitzt eine langestreckte Form (Breite 30 $\mathrm{km}$ ) von insgesamt etwa $150 \mathrm{~km}$ Länge, wobei sie im Süden nach Südwesten abknickt. Hinweise darauf ergaben sich durch die Analyse der Perturbationspfeile (Abbildung 4.11).

\section{- Inhomogene Schicht 2: Anisotroper Krustenleiter}

Die Resultate der 1D-Inversion in Abbildung 5.2 zeigen sehr deutlich einen guten Leiter in der mittleren Kruste. Seine Tiefe wird so auf $14-22 \mathrm{~km}$ festgelegt und während der weiteren Modellanpassung nicht variiert. Dies erfolgt in guter Übereinstimmung mit Ergebnissen von JöDICKE (1983) und OETTINGER (1994), der den Krustenleiter mit einer richtungsabhängigen Leitfähigkeit zweidimensional modellierte. 
Aufgrund des Verhaltens des phasensensitiven Rotationswinkels (Abbildung 4.5) sowie der Phasenaufspaltung bei kurzen Perioden (Abbildung 4.14) kann auch bei dem vorliegenden Datensatz von einer Anisotropie des Krustenleiters ausgegangen werden. Die Lamellen (Breite $\approx 8 \mathrm{~km}$ ) werden einheitlich in Richtung $45^{\circ}$ gegen Nord konstruiert. Der Leitfähigkeitskontrast $40 / 2 \Omega \mathrm{m}$ ergibt sich aus der Anpassung der Freqenzgänge der Phasen (Abbildung 4.4) für kurze Perioden sowie der Größe der Phasenaufspaltung bei E- und B-Polarisation. Die Widerstände in den Hauptanisotropierichtungen sind $\rho_{\mid} \approx 4 \Omega \mathrm{m}$ und $\rho_{\perp}=21 \Omega \mathrm{m}$. Die integrierte Leitfähigkeit in Nordost-Südwestrichtung beträgt somit 2000 S.

- Der Widerstand der unteren Kruste/des oberen Mantels $(250 \Omega \mathrm{m})$ ergibt sich aus den 1D-Modellen (Abbildung 5.2).

\section{- Inhomogene Schicht 3: Richtungsabhängige Leitfähigkeit im oberen Mantel}

Ähnlich wie beim Krustenleiter (Schicht 2) ergeben sich die Parameter hier aus der Anpassung des Frequenzganges der Phasen (Abbildung 4.4), des Rotationswinkels (4.5), und der Phasenaufspaltung (Abbildung 4.15) bei langen Perioden. Die Lamellen besitzen Widerstände von $500 \Omega \mathrm{m}$ und $1 \Omega \mathrm{m}$, wodurch die Hauptwiderstände $\rho_{\|} \approx 2 \Omega \mathrm{m}$ und $\rho_{\perp} \approx 250 \Omega \mathrm{m}$ simuliert werden. Die integrierten Leitfähigkeiten betragen $20.000 \mathrm{~S}$ in West-Ostrichtung und $160 \mathrm{~S}$ in Nord-Südrichtung.

- Der Widerstand des abschließenden Halbraums von $150 \Omega$ m wird von den Daten nicht gefordert. Er wird in Anlehnung an Modelle des oberen Erdmantels von BAHR et al. (1993) und OLSEN (1998) festgelegt. 
(1)

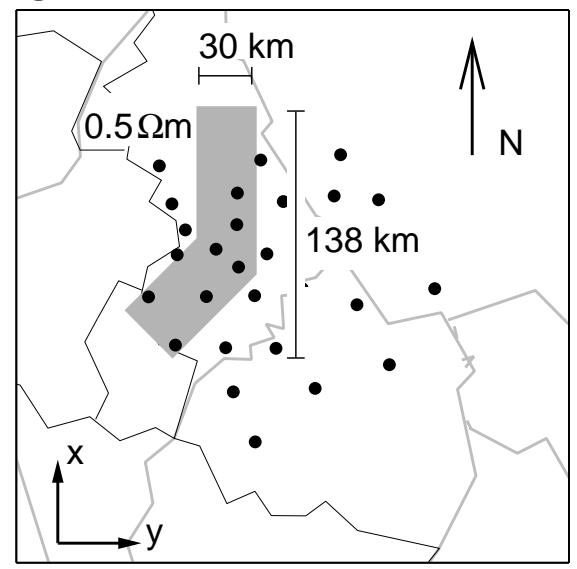

(2)

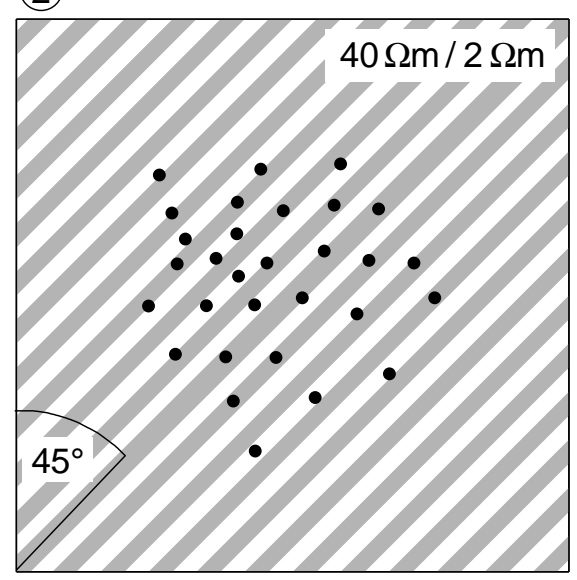

(3)

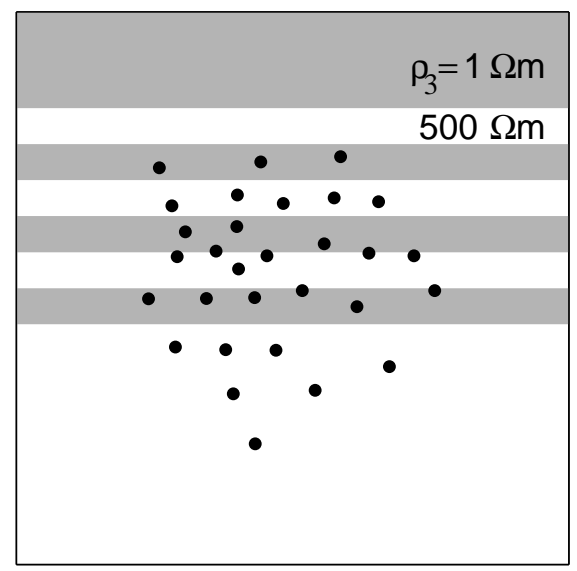

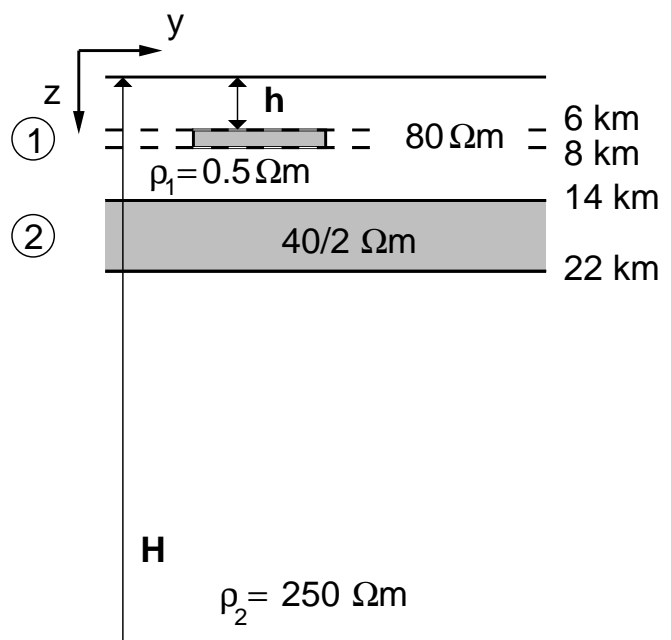

(3)

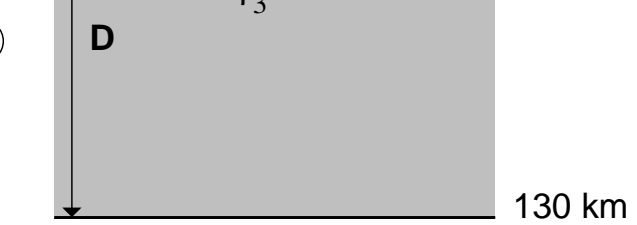

$90 \mathrm{~km}$

$130 \mathrm{~km}$

$190 \mathrm{~km}$

$150 \Omega m$

Abbildung 5.9: Die Struktur des Grundmodells. Die drei lateral inhomogenen Schichten sind in der Aufsicht abgebildet. Zur Orientierung sind in der Darstellung der ersten Schicht zusätzlich Ländergrenzen und Flüsse eingezeichnet. Der vertikale Aufbau ist linear dargestellt. Die Parameter $\mathbf{h}, \rho_{1}, \mathbf{H}, \rho_{2}, \mathbf{D}, \rho_{3}$ werden im Rahmen der Modellabgrenzung systematisch variiert. 


\subsection{Die Modellantwort}

Bei den folgenden flächenhaften Darstellungen der Modellergebnisse wird grundsätzlich nur der Wert derjenigen Modellzelle verwendet, die dem tatsächlichen Standort der Feldstation am nächsten kommt.

\subsubsection{Die Anomalie im magnetischen Feld}

Das räumliche Verhalten der magnetischen Übertragungsfunktionen $\operatorname{Re}\left\{d_{D}\right\}$ und $\operatorname{Re}\left\{z_{D}\right\}$ der Felddaten wurde in Abbildung 4.9 vorgestellt, eine entsprechende Darstellung der Modelldaten findet man in Abbildung 5.10.

- Die räumliche Ausdehnung der Anomalie im $\operatorname{Re}\left\{d_{D}\right\}$ wird gut wiedergegeben. Die Halbwertsbreite stimmt sowohl in Nord-Süd- als auch in West-Ostrichtung gut mit den Daten überein. Das lokale Maximum wird im Modell und in den Daten bei den Stationen BAD, HIL, HON erreicht.

- Bei der flächenhaften Auftragung des $\operatorname{Re}\left\{z_{D}\right\}$ wird die beobachtete Asymmetrie modelliert: Obwohl im Modell bei BOO der $\operatorname{Re}\left\{z_{D}\right\}>0.15$ wird, erreicht er nicht den Betrag der negativen Werte $\left(\operatorname{Re}\left\{z_{D}\right\}<-0.3\right)$ auf der anderen Seite der Anomalie.

Ein Vergleich der Frequenzgänge der magnetischen Übertragungsfunktionen $d_{D}$ und $z_{D}$ zwischen Modellantwort und gemessenen Daten der Station HIL ist in Abbildung 5.11 gezeigt.

- Die Frequenzgänge der magnetischen Übertragungsfunktionen werden gut angepaßt. Das modellierte Maximum im $\operatorname{Re}\left\{d_{D}\right\}$ erreicht bei der richtigen Periode (256 s) die gemessene Amplitude $(\approx 0.4)$. Der Gradient beim Abfall zu kürzeren und längeren Perioden wird allerdings nicht in der beobachteten Steilheit reproduziert. Die Modellierung des Imaginärteils gelingt über den gesamten Periodenbereich.

- Das Frequenzverhalten der vertikalen Übertragungsfunktion wird prinzipiell ebenfalls gut nachgebildet. Das Maximum im $\operatorname{Re}\left\{z_{D}\right\}$ und der Nulldurchgang vom $\operatorname{Im}\left\{z_{D}\right\}$ werden im richtigen Periodenbereich modelliert. Die Kurven des beobachteten und modellierten Real- und Imaginärteils sind jedoch um einen Faktor gegeneinander verschoben, der zu kürzeren Perioden größer wird. 

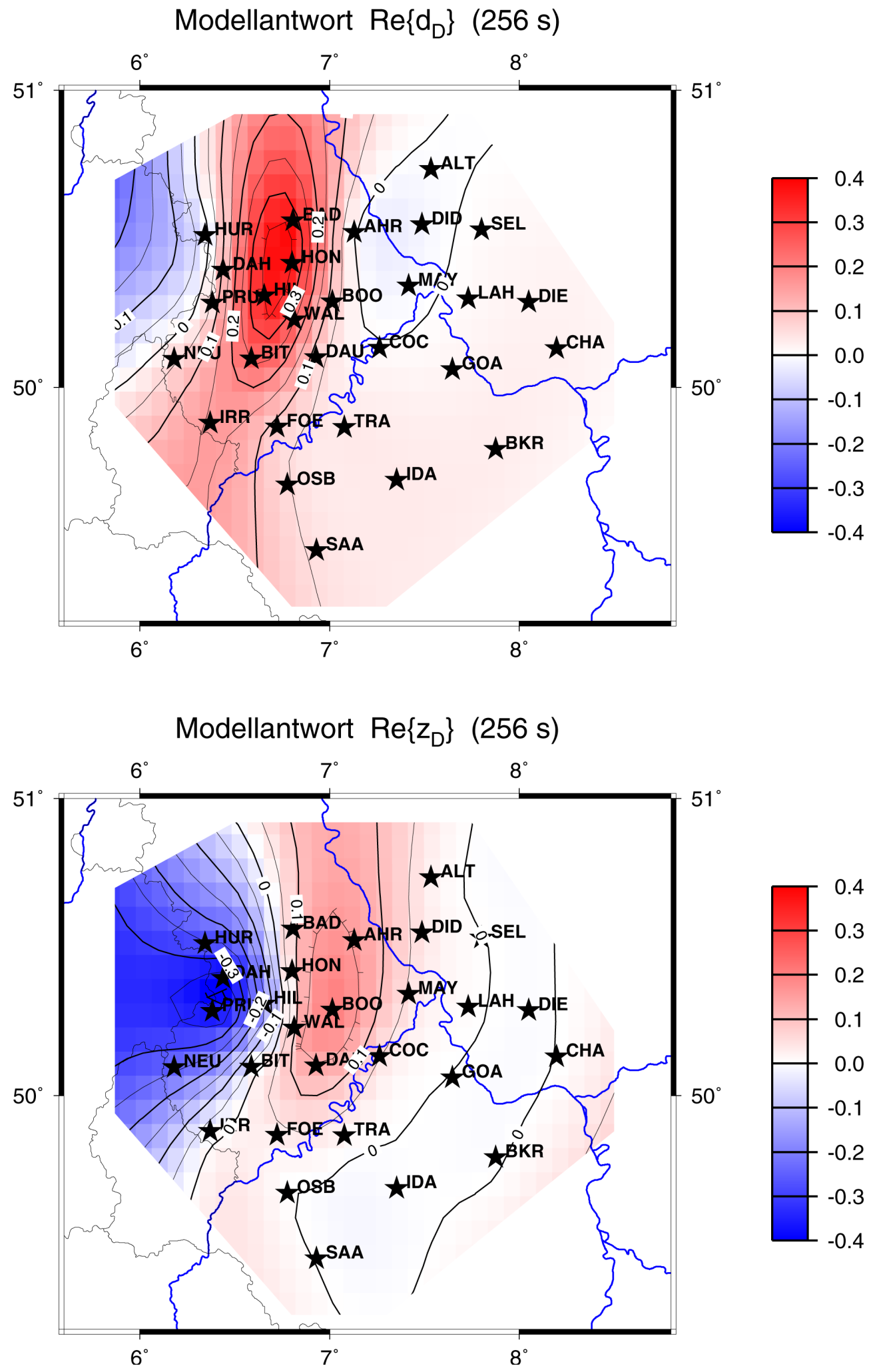

Abbildung 5.10: Flächenhafte Aufragung des $\operatorname{Re}\left\{d_{D}\right\}$ und des $\operatorname{Re}\left\{z_{D}\right\}$, berechnet für das Grundmodell bei einer Periode von 256 s. 

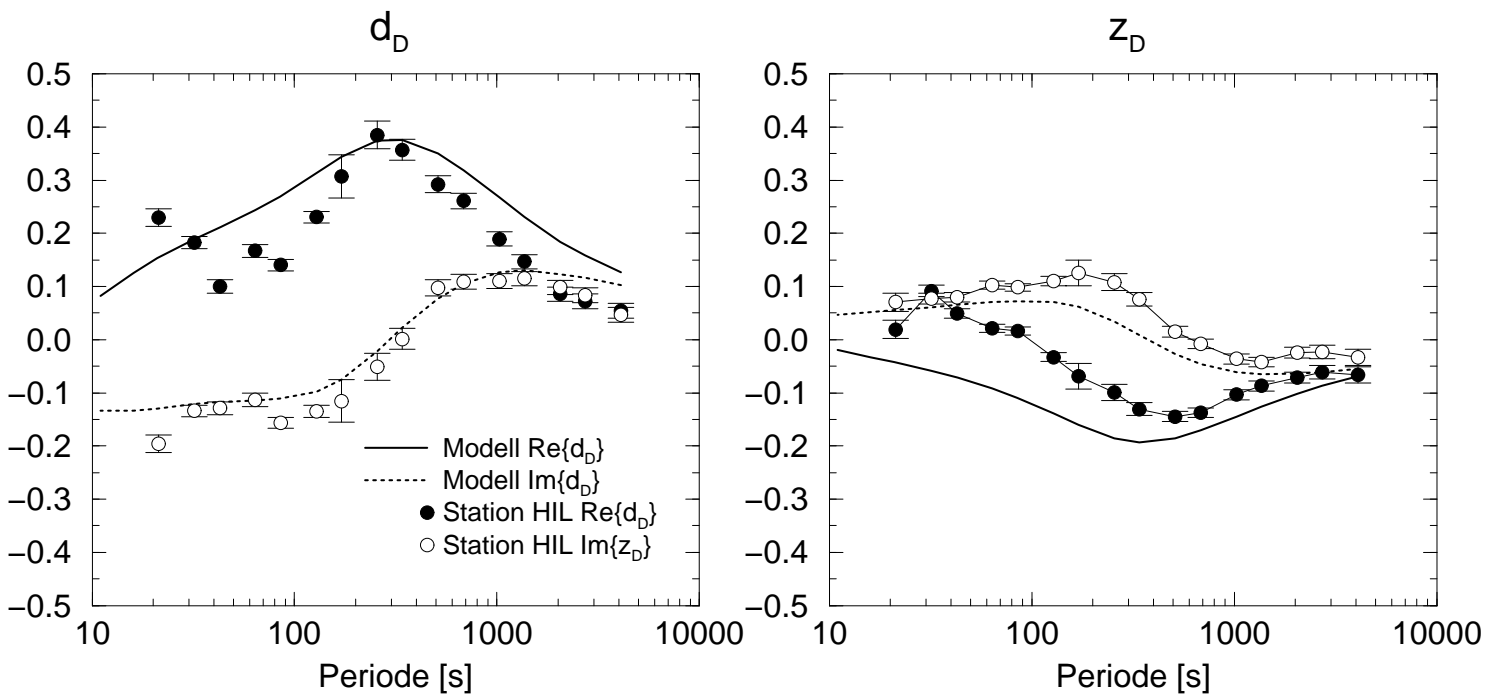

Abbildung 5.11: Vergleich der Frequenzgänge von $d_{D}$ und $z_{D}$ an der Feldstation HIL mit den entsprechenden Modelldaten.

Das Frequenzverhalten der horizontalen magnetischen Übertragungsfunktionen erweist sich als besonders sensitiv auf eine Variation der Tiefenlage der Anomalie und ihres spezifischen Widerstandes. Um die Größe dieser Modellparameter abzugrenzen, werden sie schrittweise variiert und die Veränderung im Frequenzgang vom $\operatorname{Re}\left\{d_{D}\right\}$ beobachtet $(\mathrm{Ab}-$ bildung 5.12).

Die Tiefe der Oberkante der Anomalie wird ausgehend vom Grundmodell $(6 \mathrm{~km}) \mathrm{im}$ Bereich 2 - $12 \mathrm{~km}$ verschoben. Die integrierte Leitfähigkeit der Anomalie (Mächtigkeit 2000 m) wird im Bereich 400 - 40.000 S (Grundmodell 4000 S) variiert.

Folgende Resultate werden erzielt:

- Ein Verlegen der Anomalie in größere Tiefen führt dazu, daß die an der Oberfläche beobachteten Amplituden kleiner werden und zudem erst bei größeren Perioden erreicht werden. Bei kürzeren Perioden $<100 \mathrm{~s}$ zeigt insbesondere der $\operatorname{Im}\left\{d_{D}\right\}$ ein falsches Verhalten, indem er entgegen der Beobachtung gegen null konvergiert. Auch der $\operatorname{Re}\left\{d_{D}\right\}$ fällt zu schnell ab. Eine sehr oberflächennahe Struktur dagegen würde bei kurzen Perioden einen zu starken Effekt in den Übertragungsfunktionen erzeugen. Das Frequenzverhalten von $d_{D}$ bei langen Perioden jenseits des Maximums ist dagegen recht einheitlich und wird durch ein Verschieben der anomalen Struktur in vertikaler Richtung nur unwesentlich beeinflußt.

- Bei der Variation des spezifischen Widerstandes der Anomalie fällt auf, daß bei geringer Leitfähigkeit $(<2000 \mathrm{~S})$ die beobachteten Gradienten im Frequenzgang zu gering sind. Bei deutlich erhöhten Leitfähigkeiten $(>13.000 \mathrm{~S})$ werden die Amplituden größer, die jeweiligen Maxima werden allerdings bei zu langen Perioden erreicht. Offenbar kann ein Verschieben der Struktur in größere Tiefen nicht durch 

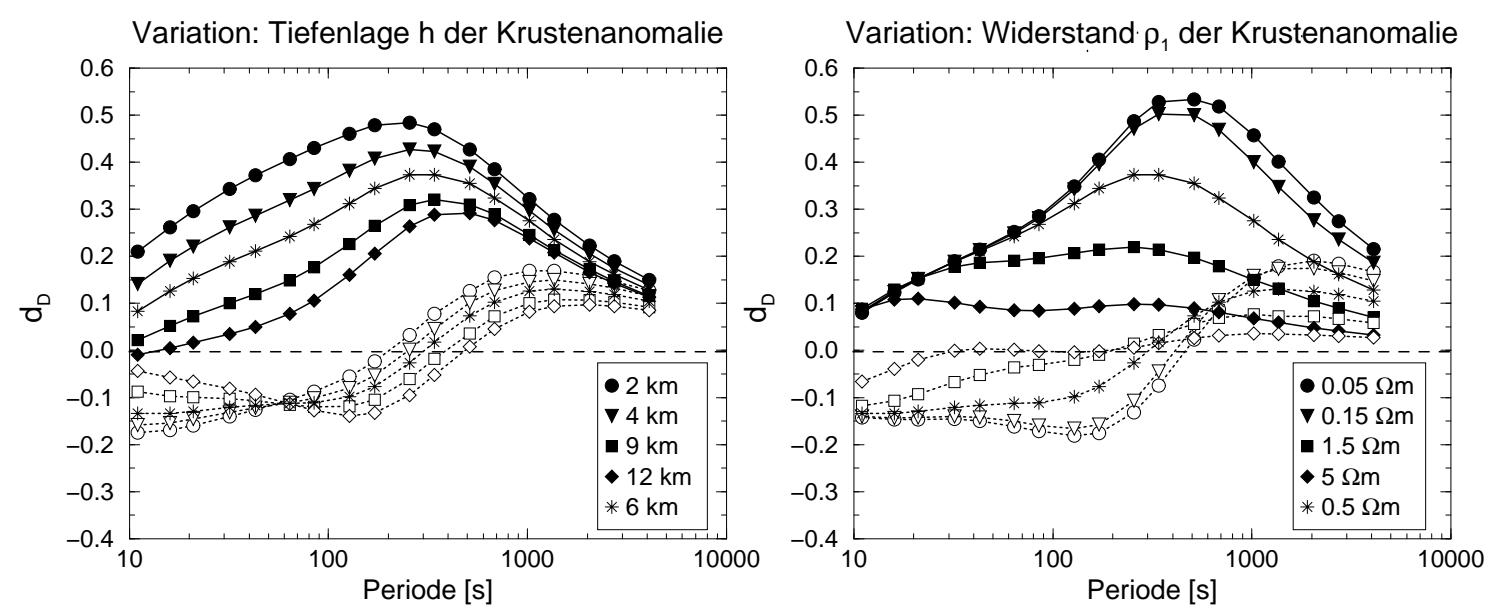

Abbildung 5.12: Variation der Tiefenlage der nordwestlichen Anomalie im Bereich 2 - 12 $\mathrm{km}$ und der integrierten Leitfähigkeit im Bereich $400-40.000 \mathrm{~S}$. Die Frequenzgänge des Grundmodells sind mit Sternen markiert $(\mathrm{h}=6 \mathrm{~km}$, $\rho_{1}=0.5 \Omega \mathrm{m}$ ). Die Mächtigkeit der Anomalie beträgt immer $2000 \mathrm{~m}$.

eine Erhöhung der Leitfähigkeit kompensiert werden, da beide Vorgänge ein Verschieben des Maximums zu längeren Perioden zur Folge haben. Ein positiver Effekt der Erhöhung der Leitfähigkeit ist die verbesserte Anpassung des Gradienten im Frequenzgang sowohl bei kurzen als auch bei langen Perioden.

\subsubsection{Die Anpassung der Phase der Impedanz}

Bei der Nachbildung des Frequenzganges der Phase wird keine spezielle Feldstation zum Vergleich herangezogen. Stattdessen wird versucht, die bei der Mehrzahl der Stationen beobachteten Merkmale zusammenzufassen und in diesem Sinne einen ,,mittleren“ Frequenzgang zu modellieren.

Die folgende Abbildung 5.13 zeigt eine exemplarische Modellantwort an der Station DIE. In unrotierten Koordinaten zeigt der Phasenverlauf das in den Feldaten beobachtete charakteristische Verhalten:

Bis etwa zu einer Periode von etwa $100 \mathrm{~s}$ liegen die Werte deutlich über $45^{\circ}$, das Maximum liegt bei $32 \mathrm{~s}$. Beide Polarisationen spalten dann im richtigen Periodenbereich auf, wobei $\phi_{x y}$ immer $<45^{\circ}$ ist und $\phi_{y x}$ ein Maximum von fast $60^{\circ}$ erreicht. Auch das Abfallen von $\phi_{y x}$ bei langen Perioden, wie es sich bei SEL, DIE, GOA und HIL andeutet, wird modelliert. Nach einer Rotation um $45^{\circ}$ laufen die Phasen bei langen Perioden zusammen und spalten im Einklang mit den Felddaten (vgl. Abbildung 4.14) bei Perioden um $100 \mathrm{~s}$ auf.

Das Frequenzverhalten des phasensensitiven Rotationswinkels wird durch das Modell sehr gut reproduziert (vgl. Abbildung 4.5). Wichtiger als die exakte Wiedergabe des richtigen Winkelbetrags (besonders für Perioden $<100 \mathrm{~s}$ weicht der modellierte Winkel mit 
$45^{\circ}$ von dem beobachteten Mittelwert der Daten $\approx 30^{\circ} \mathrm{ab}^{1}$ ) ist die Modellierung des Übergangsbereiches. Er tritt wie bei den Felddaten im mittleren Periodenbereich 100 -1000 s auf.

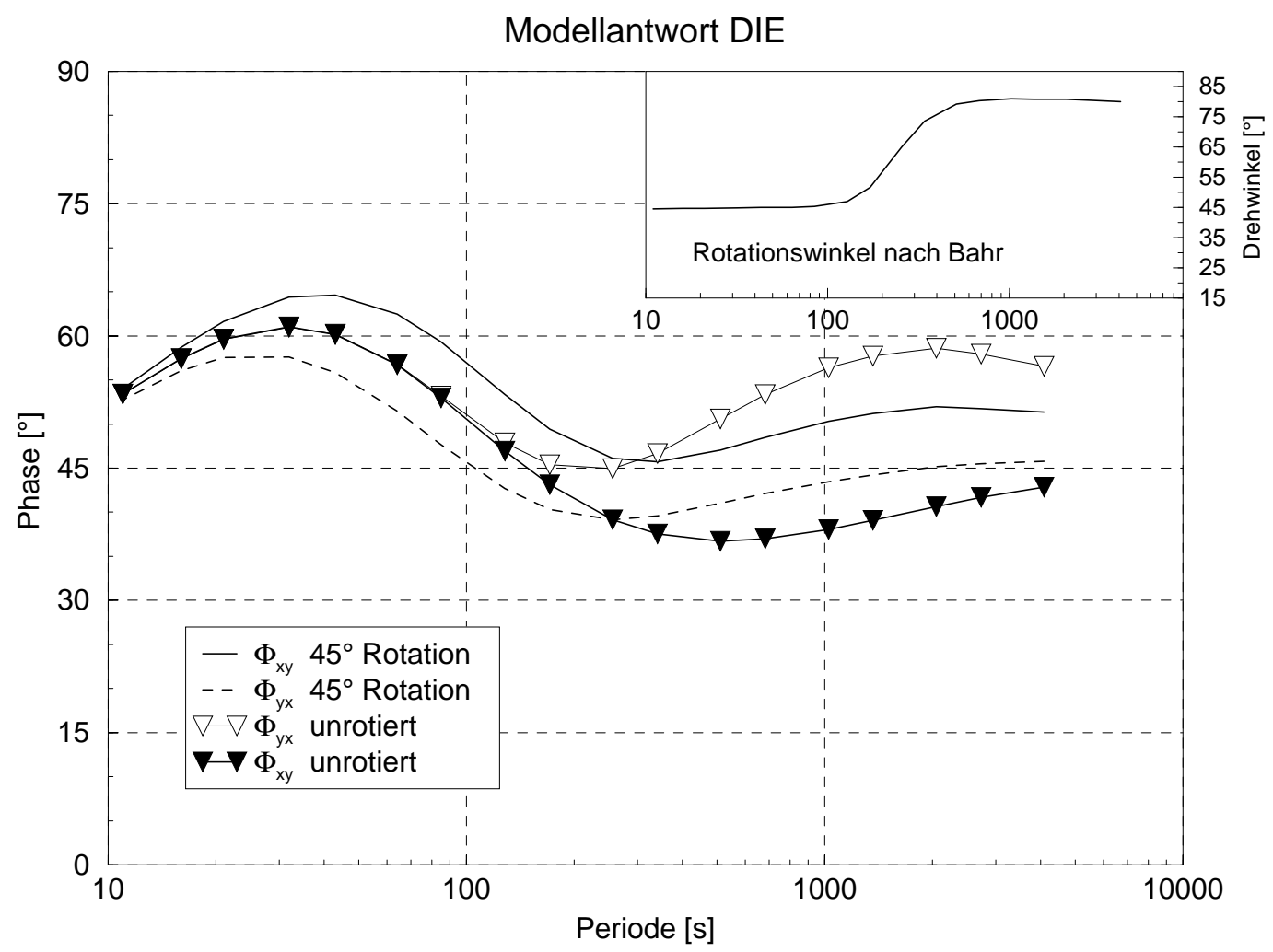

Abbildung 5.13: Der Frequenzgang der Phase der Impedanz und des phasensensitiven Rotationswinkels, berechnet für das Grundmodell an der Station DIE.

Durch das Modell sollte jedoch nicht nur der Frequenzgang, sondern auch das räumliche Verhalten der Phase bei langen Perioden wiedergegeben werden. Beim Vergleich der Felddaten (Abbildung 4.4) mit den Modelldaten in Abbildung 5.14 fällt auf, daß die wesentliche Eigenschaft der Felddaten, nämlich Wert für $\phi_{x y}$, die fast flächendeckend unter $45^{\circ}$ liegen und Phasenwerte $\phi_{y x}$, die von Süden nach Norden ansteigend Werte $>60^{\circ}$ erreichen, reproduziert wird. Laterale Strukturen, die in den Felddaten beobachtet werden, zum Beispiel das lokale Minimum in $\phi_{y x}$ im Bereich der Anomalie in der oberen Kruste, werden jedoch nicht erzeugt.

\footnotetext{
${ }^{1}$ Der Grund für den falschen Wert bei kurzen Perioden ist rein technischer Natur: Zur Modellierung der anisotropen Schicht werden Lamellen mit einheitlicher Richtung verwendet. Bei Verwendung von quadratischen Modellzellen kann so ein Winkel von $45^{\circ}$ am einfachsten konstruiert werden.
} 

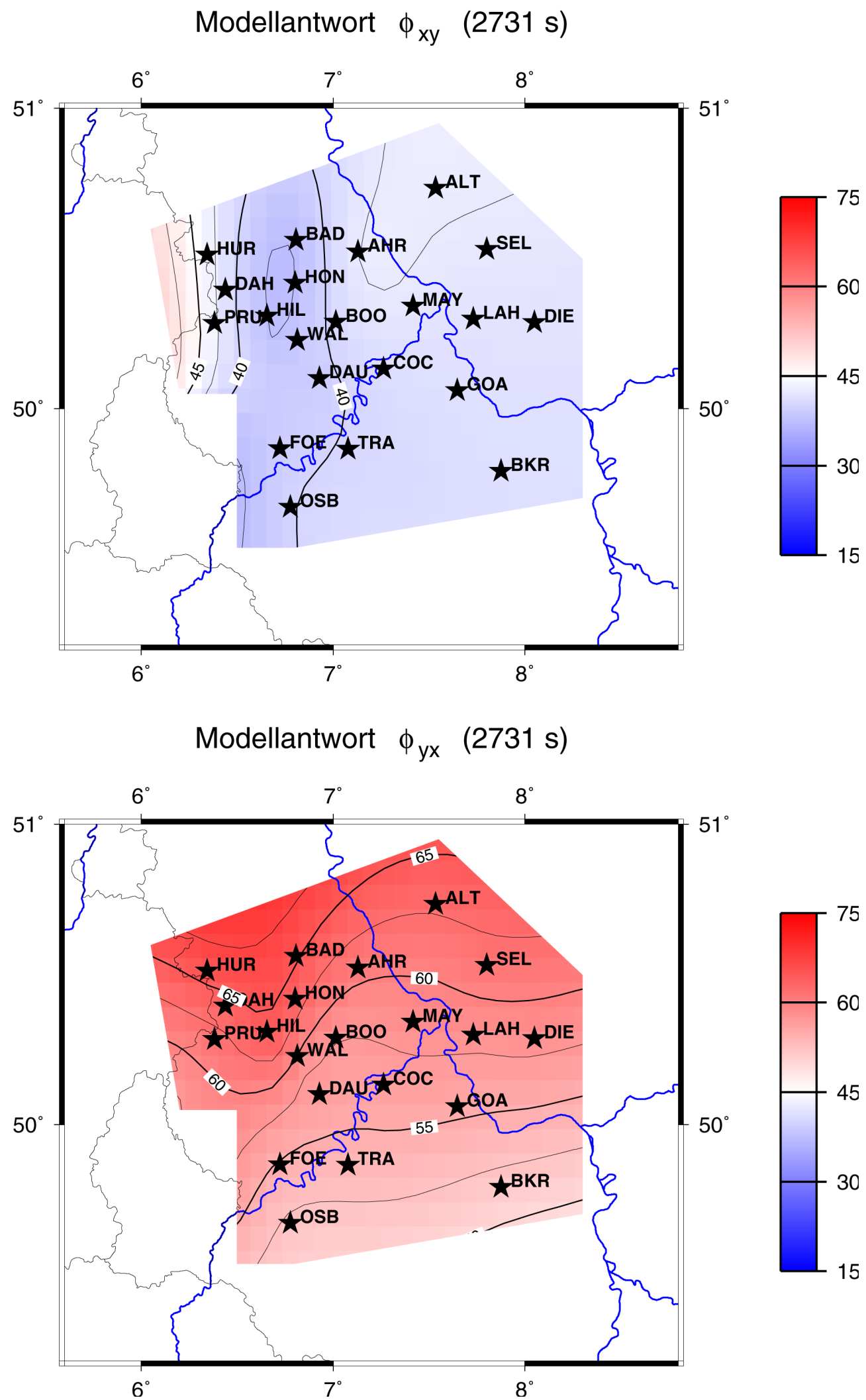

Abbildung 5.14: Flächenhafte Auftragung der Phase der Impedanz, berechnet für das Grundmodell bei einer Periode von 2731 s. 
Um weitere Hinweise auf die Struktur der Anomalie im oberen Mantel zu erhalten, werden einige Parameter, die sich als besonders sensitiv auf den Frequenzgang der Phase bei langen Perioden herausgestellt hatten, ausgehend vom Grundmodell systematisch variiert. Im einzelnen sind dies: Die Oberkante der Mantelstruktur $\mathbf{H}$ und deren integrierte Leitfähigkeit (D.0.5 S/m), der spezifische Widerstand des darüberliegenden Mantelmaterials $\rho_{2}$ sowie der spezifische Widerstand der gutleitenden Phase $\rho_{3}$. Die Ergebnisse sind in Abbildung 5.15 dargestellt.
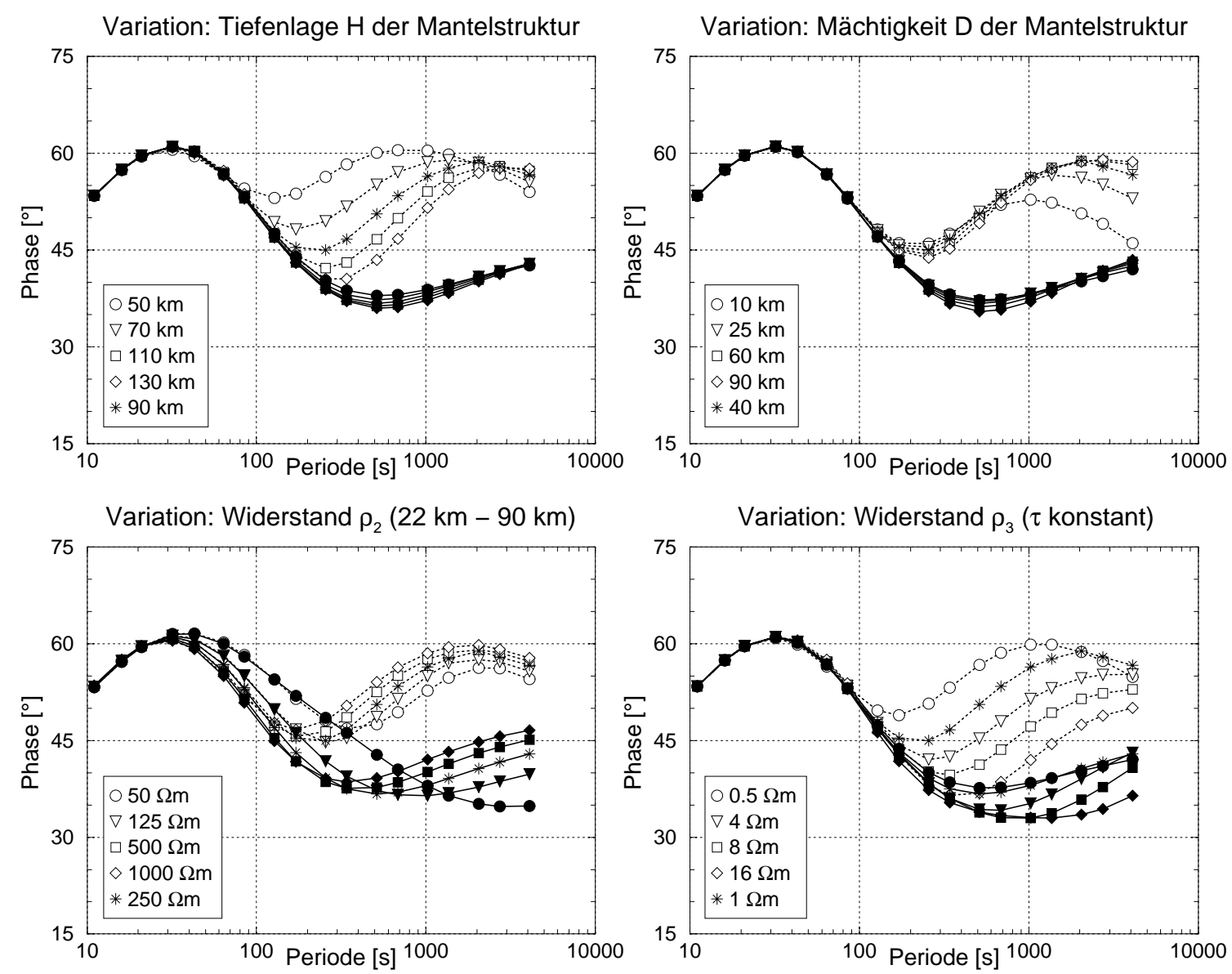

Abbildung 5.15: Einfluß der Variation von 4 Parametern (ausgehend vom Grundmodell) auf den Frequenzgang der Phase: Tiefenlage $\mathrm{H}$ der Mantelstruktur (50 130 km); Mächtigkeit D der Mantelstruktur (10 - 90 km), das entspricht einer Variation der integrierten Leitfähigkeit 5000 - $45.000 \mathrm{~S}$; Widerstand $\rho_{2}$ des obersten Mantels (50 - $1000 \Omega \mathrm{m}$; Widerstand der gutleitfähigen Phase $\rho_{3}(0.5-16 \Omega \mathrm{m})$. Der Frequenzgang des Grundmodells ist mit Sternen markiert. Schwarze Symbole kennzeichnen die $x y$-Polarisation. 
Folgende Abschätzungen können anhand der Ergebnisse gemacht werden:

- Eine Variation der Mächtigkeit D der Struktur im Bereich 10 - 90 km entspricht einer Variation der integrierten Leitfähigkeit der anisotropen Schicht in der Richtung des geringeren Hauptwiderstandes nach Gleichung 5.1 (West-Ostrichtung: $\rho_{\|} \approx 2 \Omega \mathrm{m}$ ) von 5000 - 45.000 S. Ein Einfluß macht sich nur in der $y x$-Polarisation bemerkbar: Bei einer zu geringen integrierten Leitfähigkeit $(\tau<12.500 \mathrm{~S})$ fällt $\phi_{y x}$ bei Perioden $>1000 \mathrm{~s}$ zu schnell ab, die benötigte Phasenaufspaltung wird nicht erreicht.

- Die Variation des spezifischen Widerstandes $\rho_{2}$ des Mantels oberhalb der anomalen Struktur (22 - $90 \mathrm{~km}$ ) hat besonders auf den Frequenzgang von $\phi_{x y}$ Einfluß. Falls die Leitfähigkeit zu hoch $\left(\rho_{2}<100 \Omega \mathrm{m}\right)$ ist, wird kein deutliches Minimum beobachtet, ist $\rho_{2}$ dagegen zu groß $\left(\rho_{2}>500 \Omega m\right)$, so steigt die Kurve von $\phi_{x y}$ bei langen Perioden zu stark an.

- Die Variation der Tiefenlage H der Mantelstruktur bewirkt eine Verschiebung der Periode, ab der beide Polarisationen aufspalten. Der Kurvenverlauf der $x y$-Polarisation ändert sich dabei fast nicht. Liegt die Anomalie in zu geringen Tiefen $(<70 \mathrm{~km})$, so spalten beide Polarisationen zu früh auf. Einen ähnlichen Effekt beobachtet man bei einer Variation des spezifischen Widerstandes der gut leitfähigen Phase (wobei $\tau=20.000 \mathrm{~S}$ bezogen auf $\rho_{\|} \approx 2 \Omega \mathrm{m}$ konstant bleiben soll). Hier wird allerdings auch die Kurve von $\phi_{x y}$ beeinflußt. Wird $\rho_{3}>4 \Omega \mathrm{m}$, so erreicht $\phi_{y x}$ nicht die benötigte Amplitude und auch die Kurve von $\phi_{x y}$ steigt nicht stark genug an.

\subsection{Ein lokales Modell der Leitfähigkeitsverteilung bei Station DIE}

Das im vorherigen Abschnitt vorgestellte Grundmodell beinhaltet zwei Schichten mit richtungsabhängiger Leitfähigkeit, durch die der Frequenzgang der Phase an den meisten Stationen sowie der charakteristische Verlauf der Rotationswinkel befriedigend modelliert werden kann. Eine anisotrope Schicht erzeugt jedoch kein anomales vertikales Magnetfeld, so daß das vorgestellte Modell zur Erklärung der Induktionspfeile nicht ausreicht. Im diesem und im nächsten Kapitel werden einige Vorschläge gemacht, wie das Grundmodell modifiziert werden könnte, um auch die Induktionspfeile zu reproduzieren. Der erste Schritt besteht darin, den Frequenzgang des vertikalen Magnetfeldes an einer einzelnen Station zu modellieren. Das entstandene Modell der lokalen Leitfähigkeitsverteilung bei Station DIE ist in Abbildung 5.16 gezeigt.

In einem zweiten Schritt wird dann versucht, das räumliche Verhalten der lokalen Induktionspfeile nachzubilden. 


\section{Anmerkung}

Das im folgenden beschriebene Modell wurde dazu verwendet, mit dem in Kapitel 2.4.2 vorgestellten Verfahren die magnetischen Übertragungsfunktionen auf eine eindimensionale Referenzstation zu beziehen.

Lokales Modell der Station DIE

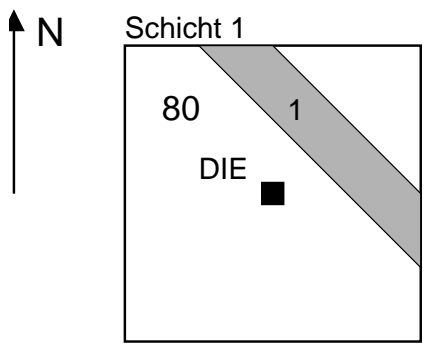

Schicht 3

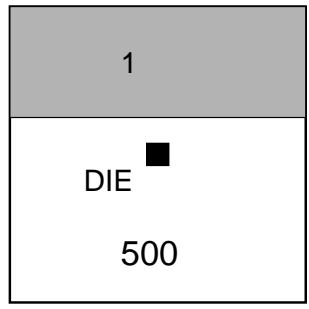

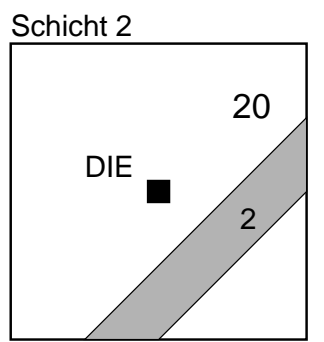

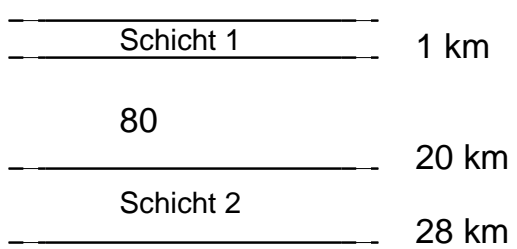

250

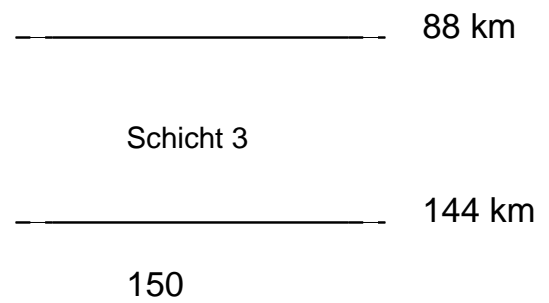

Abbildung 5.16: Lokales Modell der Station DIE. Alle Widerstände sind in $\Omega \mathrm{m}$ angegeben. Die Breite des einzelnen Dykes in Schicht 1 und 2 beträgt etwa 25 $\mathrm{km}$, in Schicht 3 etwa $100 \mathrm{~km}$.

Der wesentliche Unterschied zum Grundmodell (Abbildung 5.9) besteht darin, daß bei dem lokalen Modell keine anisotropen Schichten verwendet werden, sondern einzelne Lamellen, um die beobachteten Vorzugsrichtungen zu simulieren. Eine Lamelle wirkt als eine 2D-Anomalie und erzeugt als solche ein anomales vertikales Magnetfeld bei Anregung in E-Polarisation.

Darüber hinaus weicht das lokale Modell nur in Details ab, um spezielle Eigenheiten der Daten von Station DIE zu erklären. Die inhomogene oberste Schicht dient ausschließlich dazu, das Verhalten der Induktionspfeile bei den kürzesten Perioden zu erklären. Die Tiefenlage des Krustenleiters und der anomalen Mantelstruktur weichen geringfügig vom Grundmodell ab, ebenso die Hauptwiderstände der anisotropen Schicht in der Kruste.

Vergleiche zwischen Felddaten und Modelldaten sind in Abbildung 5.17 (lokale Induktionspfeile) und Abbildung 5.18 (scheinbarer spezifischer Widerstand und Phase der Impedanz) gegeben.

Sowohl die Realpfeile als auch die Imaginärpfeile werden bis auf die kürzesten Perioden durch das vorgeschlagene Modell sehr gut reproduziert. Besonders der Nulldurchgang 

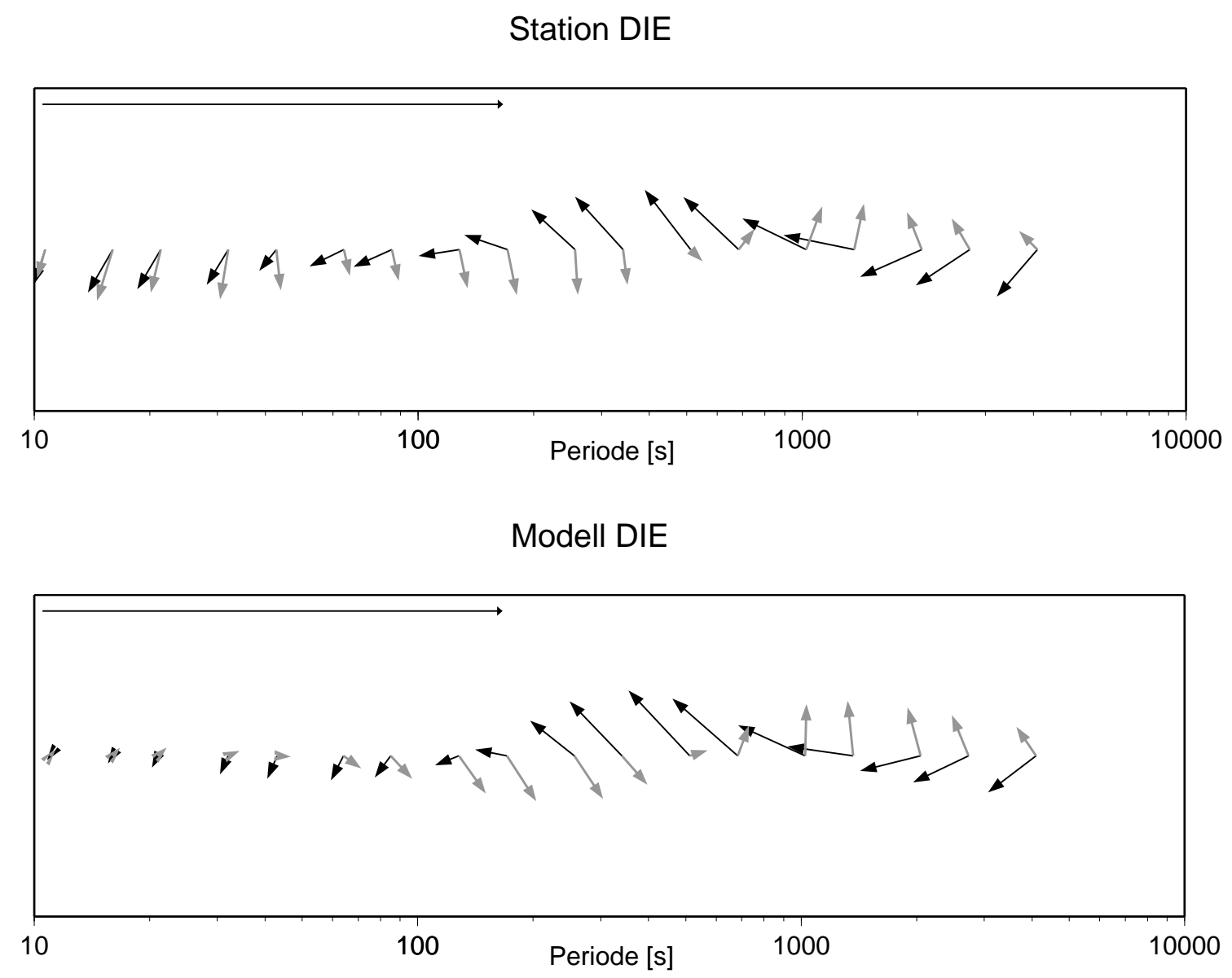

Abbildung 5.17: Station DIE und lokales Modell DIE: Lokale Induktionspfeile. Der Referenzpfeil hat die Länge eins. Die Realpfeile sind schwarz dargestellt.

des Imaginärteils bei $512 \mathrm{~s}$ und der Verlauf der Induktionspfeile bei Perioden über $2000 \mathrm{~s}$ werden fast exakt nachgebildet.

Auch der charakteristische Verlauf des scheinbaren spezifischen Widerstandes wird modelliert. Die Modellkurven sind gegenüber den Meßdaten geringfügig verschoben, zeigen aber ansonsten alle Merkmale der Daten. Hervorzuheben ist die Modellierung des Aufspaltens der beiden Polarisationen bei mittleren Perioden, das Überkreuzen der Kurven bei 1000 s und die anschließende wiederholte Aufspaltung. Der Verlauf der Phase wird in beiden Polarisationen für Perioden $>50 \mathrm{~s}$ gut wiedergegeben.

Die Zone hoher Leitfähigkeit im oberen Mantel besitzt in diesem Modell eine integrierte Leitfähigkeit von 60.000 S. Um abzuschätzen, bei welchen Perioden der Einfluß dieser Struktur abklingt, wurden Modellrechnungen mit sehr langen Perioden bis zum Tagesgang durchgeführt (Tabelle 5.1). Es zeigt sich, daß noch bei Perioden $>8$ h die Phasenaufspaltung beider Polarisationen etwa $10^{\circ}$ beträgt. Der Einfluß der Struktur auf das vertikales Magnetfeld wird ebenfalls noch bei sehr kleinen Frequenzen beobachtet: Bei $8 \mathrm{~h}$ hat der lokale Induktionspfeil eine Länge von 0.1 (Realpfeil). 

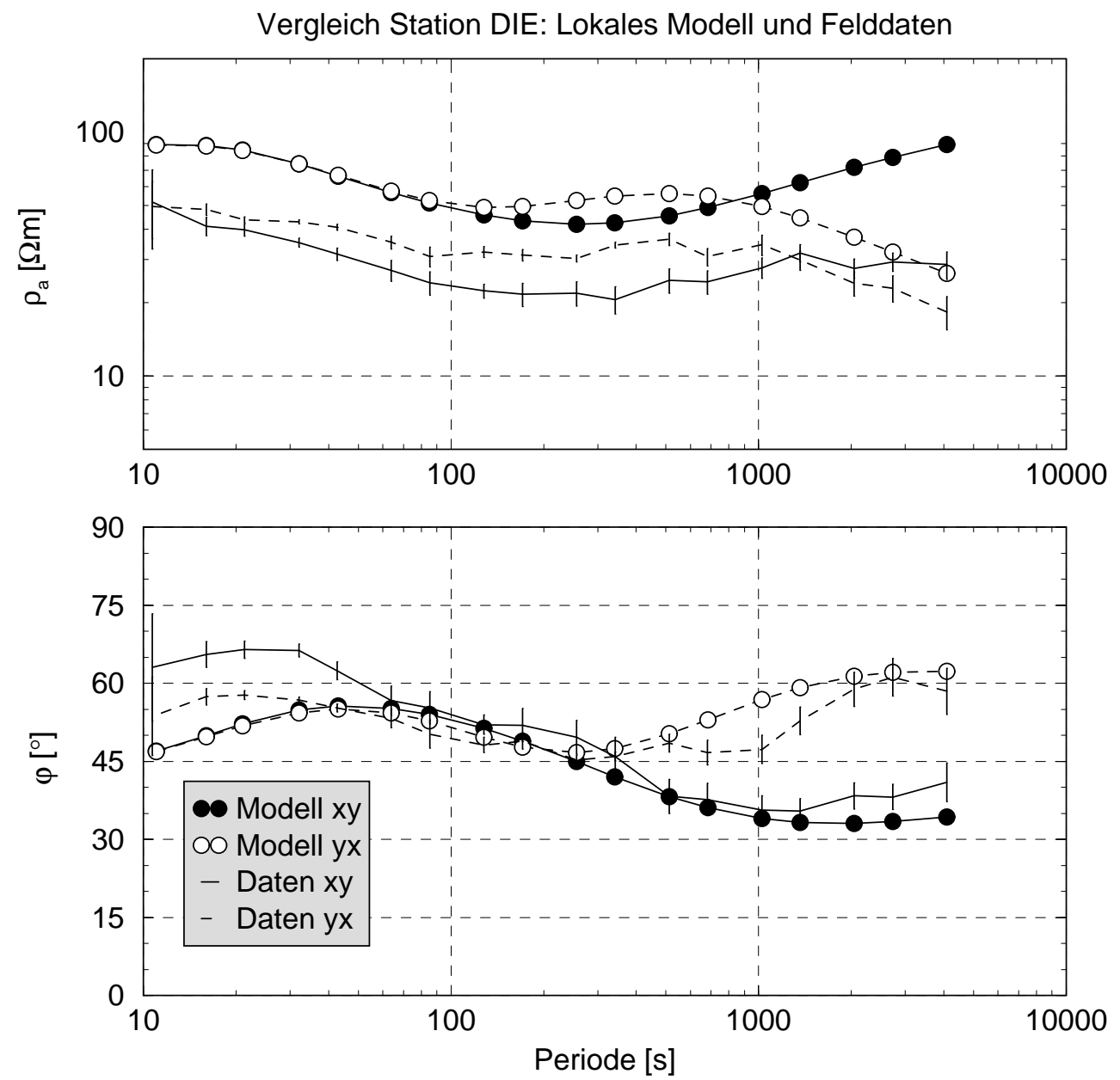

Abbildung 5.18: Station DIE und lokales Modell DIE: Phase der Impedanz und scheinbarer spezifischer Widerstand.

\begin{tabular}{|l|c|c||c|c||c|c|}
\hline Periode & $\phi(x y)\left[^{\circ}\right]$ & $\phi(y x)\left[{ }^{\circ}\right]$ & \multicolumn{2}{|c||}{ Realpfeil } & \multicolumn{2}{|c|}{ Imaginärpfeil } \\
\hline & & & Betrag & Winkel $\left[^{\circ}\right]$ & Betrag & Winkel $\left[^{\circ}\right]$ \\
\hline \hline $2 \mathrm{~h}$ & 34.8 & 59.0 & 0.12 & 228 & 0.06 & 143 \\
\hline $4 \mathrm{~h}$ & 37.1 & 55.4 & 0.11 & 237 & 0.04 & 168 \\
\hline $6 \mathrm{~h}$ & 38.3 & 52.7 & 0.10 & 241 & 0.04 & 181 \\
\hline $8 \mathrm{~h}$ & 39.1 & 50.6 & 0.10 & 243 & 0.03 & 188 \\
\hline $12 \mathrm{~h}$ & 40.0 & 47.6 & 0.09 & 246 & 0.03 & 198 \\
\hline $24 \mathrm{~h}$ & 41.3 & 43.0 & 0.08 & 251 & 0.03 & 211 \\
\hline
\end{tabular}

Tabelle 5.1: Ergebnisse der lokalen Modellierung von Station DIE bei sehr langen Perioden bis zum Tagesgang: Phase der Impedanz und lokale Induktionspfeile. Die Daten sind nicht rotiert. 


\subsection{Flächenhafte Verteilung der Induktionspfeile bei langen Perioden}

Bei der Analyse der Felddaten fiel ein besonders einheitliches räumliches Verhalten der lokalen Induktionspfeile bei langen Perioden auf (Abbildung 4.20). Dieses Verhalten wird durch das in Kapitel 5.4 beschriebene Grundmodell nicht erklärt.

Ein erster Versuch, ein genügend großes vertikales Magnetfeld zu erzeugen, besteht darin, die Lamellen in der untersten Schicht (Schicht 3 in Abbildung 5.1) zu einem einzigen Block von identischem Volumen ,zusammenzuschieben“ .

Bei zwei weiteren Modellen wird der dreidimensionale Modellkern erweitert und die Lamellen und der Block um mehr als das Doppelte der ursprünglichen Größe verlängert. Die Ausdehnung der zweidimensionalen Strukturen bei diesen Modelltypen in WestOstrichtung beträgt mehr als $600 \mathrm{~km}$. Die untere Schicht aller vier Modelle einschließlich des Grundmodells sind in Abbildung 5.19 skizziert.

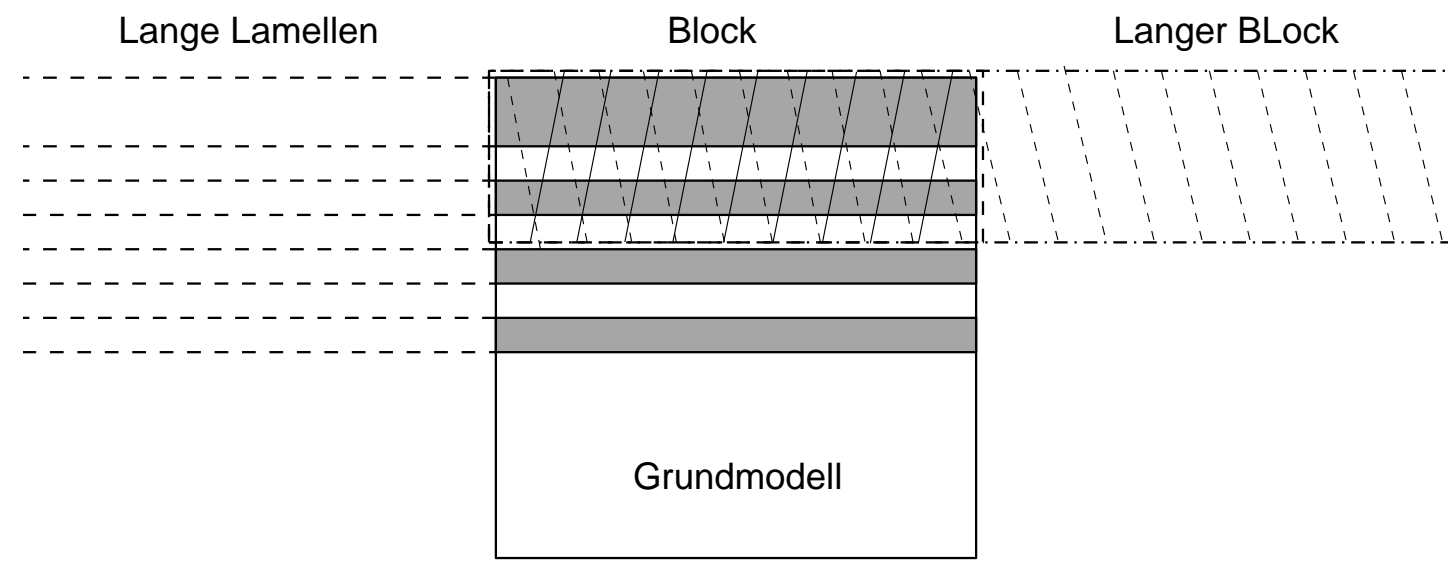

Abbildung 5.19: Aufsicht auf die unterste inhomogene Schicht des Grundmodells und drei weiterer Modellstudien.

Es zeigt sich, daß weder durch das einfache Blockmodell noch durch die verlängerten Lamellen die benötigten Amplituden im vertikalen Magnetfeld erzeugt werden können. Dies gelingt ansatzweise durch den Modelltyp „Langer Block“, wie man in Abbildung 5.20 erkennen kann.

Beim Vergleich mit den Daten (Abbildung 4.20) zeigt sich, daß die lokalen Induktionspfeile im westlichen Teil des Arrays gut reproduziert werden. Im östlichen Teil weichen die Richtungen deutlich ab, die Längen der modellierten Realpfeile erreichen jedoch immerhin bis zu $50 \%$ der tatsächlich beobachteten. 
Modell "Langer Block": Lokale Induktionspfeile (2731 s)

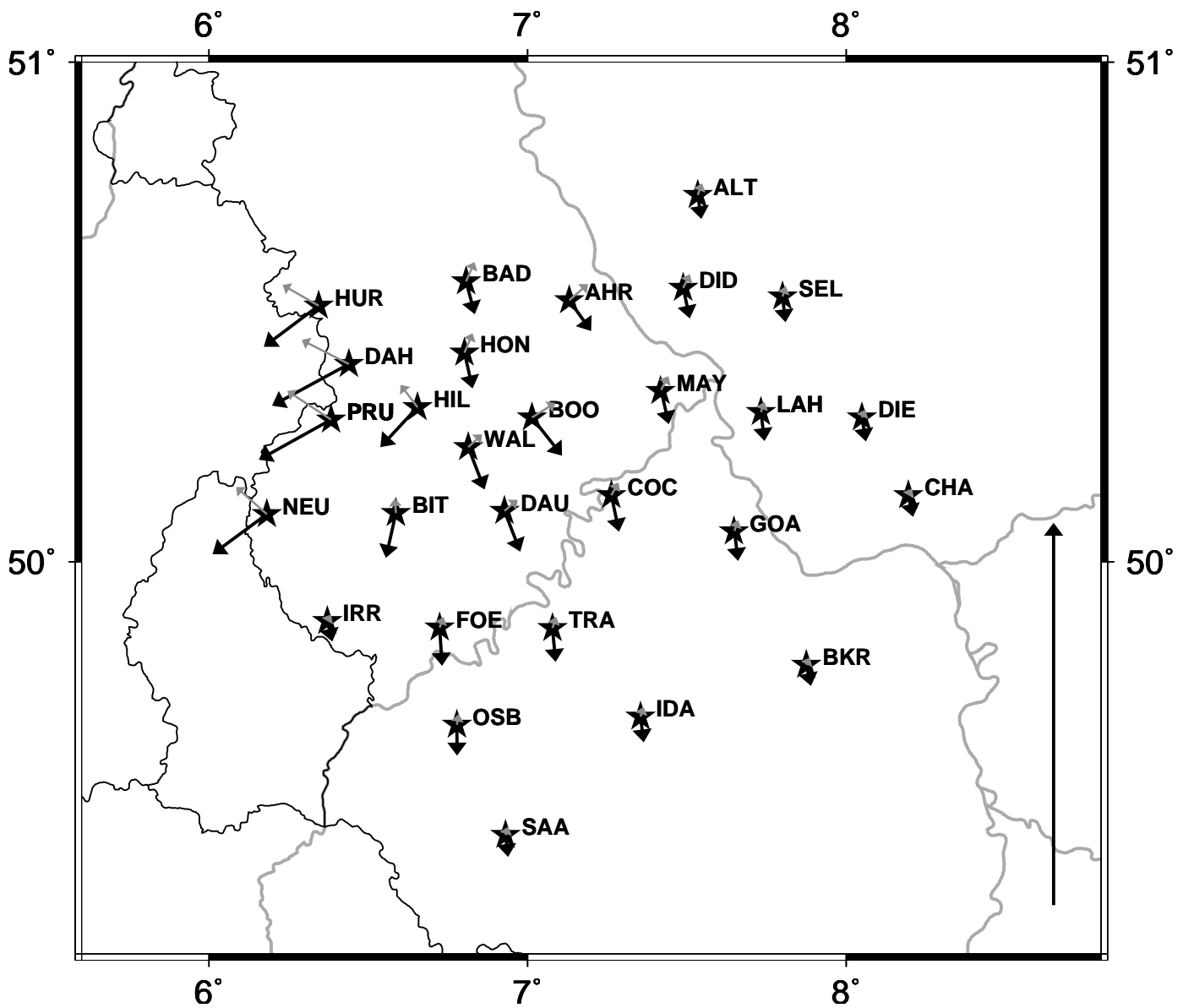

Abbildung 5.20: Flächenhafte Darstellung der lokalen Induktionspfeile, berechnet für das Modell „Langer Block“ bei der Periode 2731 s. Der eingezeichnete Referenzpfeil hat die Länge eins. Die Realpfeile sind schwarz dargestellt.

Ein Nachteil der Modelle vom „Block“-Typ wird in Abbildung 5.21 sichtbar. Hier ist die Phasenaufspaltung $\left(\phi_{y x}-\phi_{x y}\right)$ längs eines Nord-Südprofiles aufgetragen, und zwar die tatsächlich beobachtete und die aus den vier vorgstellten Modellen berechnete.

Die Phasenaufspaltung besitzt im Süden des Meßgebiets (BKR) ihr Minimum $\left(3^{\circ}\right.$ ) und läßt dann einen ansteigenden Trend in Richtung Norden erkennen, obwohl das Maximum etwa in der Mitte des Profils bei Station DIE erreicht wird $\left(23^{\circ}\right)$.

Aus dem Vergleich mit den Modelldaten geht bervor, daß durch einen einzigen Block die benötigte Phasenaufspaltung nicht erzeugt wird. Das verwendete Lamellenmodell ergibt einen vernünftigen Mittelwert, und durch die verlängerten Lamellen ist es möglich, die Aufspaltung noch zu vergrößern. 


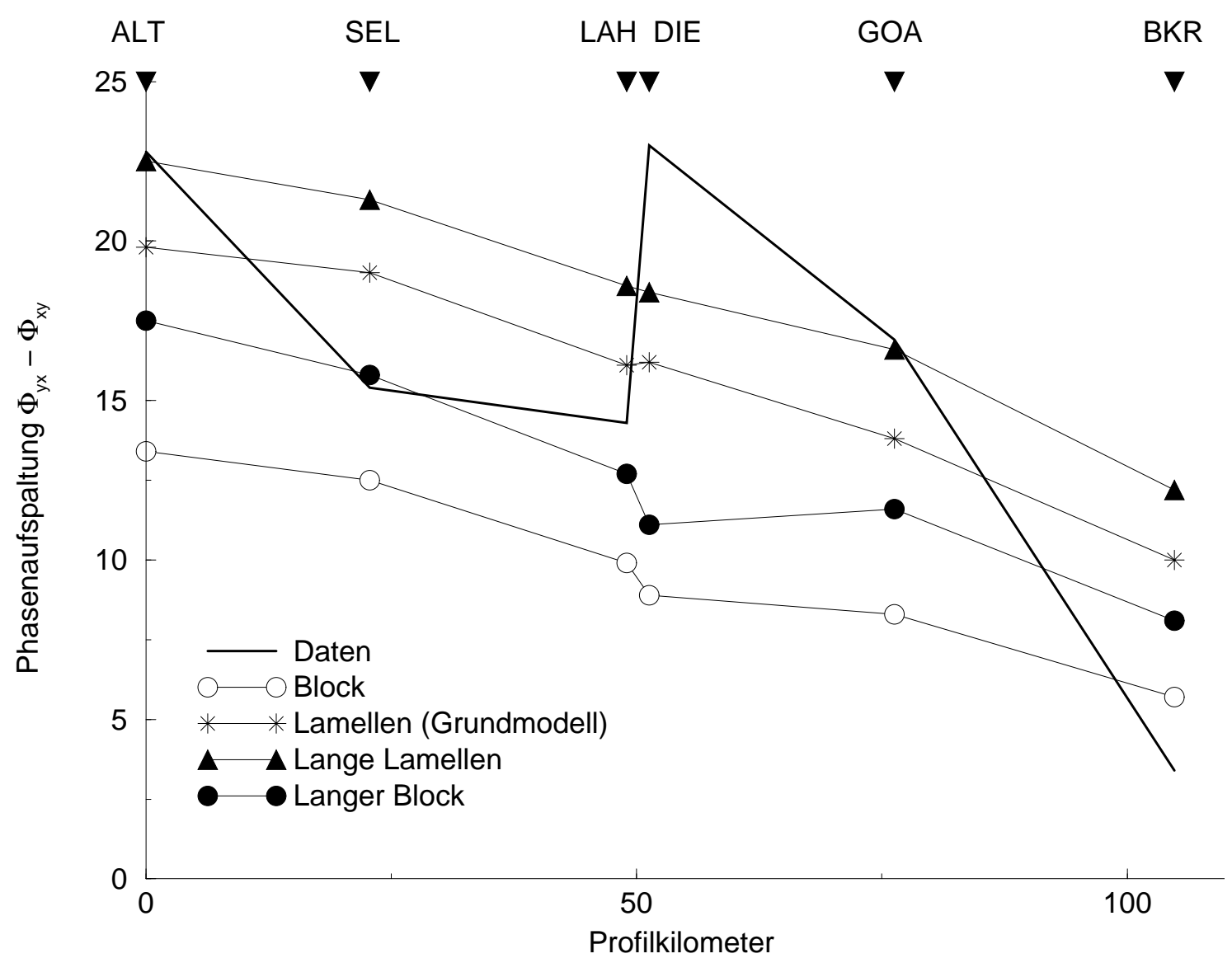

Abbildung 5.21: Phasenaufspaltung längs eines Nord-Süd Profiles, berechnet aus den vier vorgestellten Modellen sowie die tatsächlich beobachteten Werte. Alle Daten sind unrotiert. 


\section{Diskussion}

\section{Die Kruste}

Die eindimensionalen Inversionsrechnungen ergeben deutliche Hinweise auf eine gut leitfähige Zwischenschicht in der unteren Kruste in Tiefen zwischen 15 und 25 km. Mehrere Untersuchungen zeigen, daß eine solche Zone nicht ungewöhnlich ist und sogar weltweit beobachtet wird. Man geht davon aus, daß ein Bereich mit geringem Widerstand in der unteren Kruste eher die Regel als die Ausnahme ist. (HAAK \& HUTTON, 1986; JoNES, 1992). Die beobachteten mittleren Leitfähigkeiten liegen im Bereich 300 - 1500 S. Eine richtungsabhängige Leitfähigkeit, wie sie im Rheinischen Schiefergebirge beobachtet wird, tritt ebenfalls in anderen Gebieten auf (KELLETT et al., 1992; EISEL \& BAHR, 1993).

Die Natur dieser gut leitfähigen Zone ist noch nicht geklärt. Obgleich der spezifische Widerstand stark von der Temperatur abhängt, reicht der Temperaturanstieg in der Tiefe in inaktiven Regionen nicht aus, um die beobachteten Leitfähigkeiten zu erklären.

Allgemein anerkannt ist inzwischen die Modellvorstellung eines hoch leitfähigen Mediums innerhalb einer schlecht leitfähigen Gesteinmatrix (VAN'YAN \& HYNDMAN, 1996). Welches Material für die gut leitfähige Phase in Frage kommt, wird allerdings noch kontrovers diskutiert. Das Interesse konzentriert sich dabei im wesentlichen auf zwei Möglichkeiten:

Salinare Fluide oder hoch leitfähige Minerale wie Graphit, Fe / Ti-Oxide, Sulfide, die verbundene Leiterbahnen bilden (GLOVER \& VINE, 1994).

Als Quelle für freies Wasser in der unteren Kruste schlagen VAN'YAN \& GLIKO (1999) Dehydration von Amphiboliten etwa durch eine Temperaturerhöhung durch einen Mantelplume vor. Eine Akkumulation von freien Fluiden könnte in Zonen mit hoher horizontaler und geringer vertikaler Permeabilität stattfinden (BAILEY, 1990). Einige Autoren vermuten, daß wässrige Fluide in der unteren Kruste sowohl die gut leitfähige Schicht, als auch Zonen erhöhter seismischer Reflektivität erklären könnten (GOUGH, 1986; HYNDMAN \& SHEARER, 1989; MARQUIS \& HYNDMAN, 1992; KISSIN, 1996). Allerdings zeigen SIMPSON \& WARNER (1998), daß diese Bereiche nicht unbedingt übereinstimmen müssen. Darüberhinaus weisen YARDLEY \& VALLEY (1997) und MARKL \& BUCHER (1998) darauf hin, daß eine verbundene Phase freier Fluide aus petrologischen Gründen in der unteren Kruste nicht stabil, bzw. innerhalb geologischer Zeiträume nur in sehr geringen Mengen dauerhaft vorhanden sein kann .

Kohlenstoff wird als alternative Möglichkeit zur Erhöhung der Leitfähigkeit angesehen 
(Frost et al., 1989; MAREschal et al., 1992; ZhAMALETdinov, 1996). Graphit hat eine sehr hohe Leitfähigkeit von $10^{4}-10^{5} \mathrm{~S} / \mathrm{m}$, so daß seine Volumenkonzentration etwa um einen Faktor 100 - 1000 geringer als die des Fluids sein kann, um den gleichen Gesamtwiderstand zu erhalten. Auch im Rheinischen Schiefergebirge wird Graphit zur Erklärung der hohen integrierten Leitfähigkeit in der unteren Kruste herangezogen (JÖDICKE et al., 1983).

In den vorliegenden Daten wird außerdem eine Anisotropie der elektrischen Leitfähigkeit beobachtet. Sie kann mit Hilfe eines Lamellenmodells für die untere Kruste erklärt werden, dessen Hauptwiderstände sich etwa um einen Faktor 5 unterschieden (2000 S in Nordost-Südwestrichtung, $380 \mathrm{~S}$ senkrecht dazu).

Alle Modellvorstellungen des Leitfähigkeitsmechanismus müssen auch eine Erklärung für diesen Sachverhalt bieten. BAHR (1997) betrachtet ein Zweiphasensystem, das aus der Gesteinsmatrix und einem gut leitenden Material besteht und modelliert den Leitfähigkeitsmechanismus mit Hilfe von eingebetteten Netzwerken. Eine Anisotropie der elektrischen Leitfähigkeit kann durch unterschiedliche Konnektivitäten der gutleitenden Phase in verschiedene Richtungen erzeugt werden.

Im Nordwesten des Meßgebiets wird eine sehr markante Anomalie im magnetischen Feld beobachtet. Modellrechnungen zeigen, daß deren Ursache in der oberen Kruste zu suchen ist. Die Oberkante der Leitfähigkeitsanomalie wird wahrscheinlich nicht tiefer als etwa 8 - $10 \mathrm{~km}$ liegen, und ihre integrierte Leitfähigkeit beträgt mindestens 3000 - $4000 \mathrm{~S}$. Die Struktur hat eine Länge von mindestens 100 - $150 \mathrm{~km}$ bei einer Breite von etwa $30 \mathrm{~km}$. Die Mächtigkeit kann nicht genau abgeschätzt werden. Vergleichende Modellrechnungen zeigen aber, daß die Ausdehnung in vertikaler Richtung begrenzt ist. Eine Vergrößerung der Struktur bis in die untere Kruste bei Beibehaltung der integrierten Leitfähigkeit erzeugt beispielsweise nur im Zentrum der Anomalie ein anomales Feld der beobachteten Größenordnung. Man kann daher vermuten, daß die Leitfähigkeitsanomalie eine recht flache Form besitzt.

Als Ursache dieses räumlich klar begrenzten Bereiches hoher Leitfähigkeit kommen grundsätzlich ebenso die oben im Zusammenhang mit dem Krustenleiter dargelegten Möglichkeiten in Frage.

Spannungsmessungen im Linksrheinischen Schiefergebirge ergeben als Richtung der maximalen Dehnung etwa Nordost-Südwest (BAUMAnN \& ILliES, 1983). Risse und Spalten in der Lithosphäre entstehen bevorzugt senkrecht dazu, was als Erklärung für die Orientierung der Vulkanreihen senkrecht zu der variszischen Streichrichtung angesehen wird. Die Krustenanomalie ist wahrscheinlich nicht exakt Nord-Süd ausgerichtet, wenngleich sie vereinfacht so modelliert wurde. Aus der Analyse der magnetischen Daten ergibt sich eine Streichrichtung von $-17^{\circ}$ gegen Nord, was recht gut mit der Orientierung der Vulkanfelder übereinstimmt.

Eine Interpretation als Magmakammer erscheint angesichts des vermuteten Volumens der Leitfähigkeitsanomalie $\left(\approx 9000 \mathrm{~km}^{3}\right)$ unwahrscheinlich. Messungen der Wärmeflußdichte zeigen kein anomales Verhalten im Bereich der Eifel und geben ebenfalls keine Hinweise auf das Vorhandensein von partiellen Schmelzen in der Kruste (CLAUSER, 1998). 
Allerdings zeigen Modellrechnungen am Beispiel einer vermuteten Magmakammer unter dem Laacher See (Volumen $17 \mathrm{~km}^{3}$ ), daß deren Wärmeflußsignal nach 10.000 Jahren die Oberfläche noch nicht erreicht hat (HAENEL, 1983).

Ein ausgedehnter Bereich von heißen, salinaren Fluiden, die sich in Spalten und Rissen sammeln, erscheint als die wahrscheinlichste Erklärung für die Anomalie.

\section{Der obere Mantel}

Der modellierte Widerstand des oberen Mantels im Hintergrundmodell liegt mit $250 \Omega \mathrm{m}$ im Bereich der Leitfähigkeit, die für Olivin unter Mantelbedingungen vorhergesagt wird (HiRsCH \& WANG, 1986; CONSTABLE et al., 1992; XU et al., 1998).

Aus den Daten ergeben sich allerdings Hinweise auf eine Zone im oberen Mantel, die deutlich höhere Leitfähigkeiten aufweist. Solche Bereiche mit geringem Widerstand in Tiefen zwischen $50 \mathrm{~km}$ und $200 \mathrm{~km}$ werden in Europa oft beobachtet (HJELT \& KORJA, 1993) und üblicherweise mit der Übergangszone Lithosphäre/Asthenosphäre in Verbindung gebracht. Für das Rheinische Schiefergebirge geben PRAUS et al. (1990) eine Tiefe von $60 \mathrm{~km}$ an, die sie aus magnetotellurischen und seismologischen Untersuchungen ableiten.

Bei den vorliegenden Daten kann anhand der Phasenaufspaltung im Frequenzgang vermutet werden, daß die Oberkante der Leitfähigkeitsanomalie bei 60 - $70 \mathrm{~km}$ oder tiefer liegt, was mit den oben genannten Ergebnissen in Einklang steht.

Es stehen mehrere Erklärungsmöglichkeiten für eine stark erhöhte Leitfähigkeit im Erdmantel zur Diskussion.

Aufgeschmolzenes Gesteinsmaterial besitzt Leitfähigkeiten von bis zu 10 S/m (S ATO \& IDA, 1984). Allerdings wird in der Regel nicht das gesamte Material aufgeschmolzen sein, sondern in Form von partiellen Schmelzen vorliegen. Deren Leitfähigkeiten hängen vom Volumenanteil der Schmelze und der Interkonnektivität ab. Abschätzungen des effektiven spezifischen Widerstandes von partiellen Schmelzen liefern Werte, die in der Regel im Bereich von $1 \Omega \mathrm{m}$ bis zu einigen $10 \Omega \mathrm{m}$ liegen. (SATO \& IDA, 1984; SCHMELING, 1986; RoBERTS \& TYBURCZY, 1999). Auch Kohlenstoff in amorpher Form oder als Graphit (DUBA \& SHANKLAND, 1982) oder wässrige Fluide (MIBE et al., 1998) können für eine Erhöhung der Mantelleitfähigkeit sorgen, sofern sie als verbundene Phasen vorliegen. Migration von Wasserstoffionen im Olivin kann ebenfalls zu einer Erhöhung der Leitfähigkeit führen (KARATO, 1990; BAI \& KoHLSTEDT, 1992; LIZARRALDE et al., 1995).

Unter dem Rheinischen Schiefergebirge vermuten RAIKES \& BONJER (1983) eine Zone mit partiellen Schmelzen aufgrund von seismologischen Messungen. Dies widerspricht nicht den gravimetrischen Ergebnissen, die im westlichen Rheinischen Schiefergebirge eine negative Bouguer-Anomalie ergeben (JАКОВY et al., 1983). Auch die Daten der vorliegenden Arbeit können mit der Annahme von partiellen Schmelzen im Mantel, das heißt mit Leitfähigkeiten, die $1 \mathrm{~S} / \mathrm{m}$ nicht übersteigen, befriedigend erklärt werden.

Wichtigstes Merkmal des vorliegenden Datensatzes ist jedoch die beobachtete Richtungs- 
abhängigkeit der Leitfähigkeit im oberen Mantel. Entsprechende Beobachtungen in anderen Regionen finden sich bisher nur vereinzelt (JONES, 1993; KURTZ et al., 1993).

Die durchgeführten Modellrechnungen ergeben, daß die integrierte Leitfähigkeit der Zone in West-Ostrichtung mindestens $12.500-15.000 \mathrm{~S}$ beträgt (mit $\rho_{\|} \approx 2 \Omega \mathrm{m}$ und $\rho_{\perp} \approx$ $250 \Omega \mathrm{m})$.

Durch eine Variation des spezifischen Widerstandes und der Schichtmächtigkeiten bei Beibehaltung der integrierten Leitfähigkeit (20.000 S) und deren Einfluß auf den Frequenzgang der Phase kann eine Mindestleitfähigkeit von rund $0.2 \mathrm{~S} / \mathrm{m}$ der gut leitfähigen Phase abgeschätzt werden.

Die im Lamellenmodell vorgegebenen Hauptwiderstände differieren in zueinander senkrechter Richtung um einen Faktor $\approx 100$. Die Leitfähigkeit von Olivinkristallen ist zwar richtungsabhängig, die experimentell beobachtete Anisotropie von trockenem Olivin ist jedoch viel kleiner $(\approx 2)$ (Hirsch \& WANG, 1986; Constable et al., 1992). Möglicherweise könnte die Anisotropie der Diffusivität von Wasserstoff im Olivin hier eine weitere Erklärungsmöglichkeit bieten (HIRSCH, 1990). Sie kann in den verschiedenen Kristallrichtungen um einen Faktor 100 differieren (MACKWELL \& KOHLSTEDT, 1990).

Aus den Daten kann nicht ermittelt werden, ob es sich bei der beobachteten Leitfähigkeitsanomalie um eine anisotrope Schicht handelt, oder um eine großräumige zweidimensionale Struktur. Das Vorhandensein eines markanten, einheitlichen vertikalen Magnetfeldes spricht für die letztere Möglichkeit. Modellrechnungen haben gezeigt, daß sich anomale Magnetfelder der beobachteten Größenordnung bei langen Perioden nur mit ausgedehnten Strukturen von einigen hundert Kilometern Länge erzeugen lassen, es sei denn, man läßt Leitfähigkeiten deutlich $>1 \mathrm{~S} / \mathrm{m}$ zu. Die Ausdehnung des anomalen Bereiches kann mit den Stationen des vorliegenden Meßarrays nicht sicher abgegrenzt werden.

Lokale Studien, die die Daten einer einzelnen Station erklären sollen, machen deutlich, daß Leitfähigkeitskontraste im Mantel in der Größenordnung 500/1 $\Omega \mathrm{m}$ ausreichend sind, um sowohl die beobachteten lokalen Induktionspfeile als auch die magnetotellurischen Parameter zu modellieren. Ein genügend großes vertikales Magnetfeld entsteht jedoch nur bei der Verwendung von einzelnen 2D-Strukturen, wodurch wiederum die Anpassung der flächenhaften Phasenaufspaltung verschlechtert wird.

Zur Erklärung aller Datenmerkmale müßte ein Weg gefunden werden, die Eigenschaften beider Modelltypen zu kombinieren. Möglicherweise gelingt dies durch die Verwendung von unregelmäßigen, gebogenen oder mit unterschiedlichen Leitfähigkeiten versehenen Lamellen. Die beobachteten Induktionspfeile bei langen Perioden könnten darüberhinaus von Strukturen beinflußt sein, die bereits (bzw. auch) außerhalb des modellierten Bereiches liegen ${ }^{2}$.

\footnotetext{
${ }^{2}$ Es ist unwahrscheinlich, daß die beobachteten anomalen vertikalen Magnetfelder von den sehr gut leitenden Sedimenten der norddeutschen Tiefebene (die sogenannte norddeutsche Leitfähigkeitsanomalie) herrühren. Sie erzeugen zwar ein starkes vertikales Magnetfeld. Modellabschätzungen lassen aber vermuten, daß es im Gebiet der Eifel zum größten Teil abgeklungen ist (JUNGE, 1994).
} 
Die Ergebnisse der elektromagnetischen Tiefenforschung lassen eine oder mehrere großräumige Zonen erhöhter elektrischer Leitfähigkeit unter dem Rheinischen Schiefergebirge vermuten. Es wird angenommen, daß sie in groben Zügen eine zweidimensionale Struktur aufweisen, um gleichzeitig eine Aufspaltung der Polarisationen der Phase der Impedanz und ein anomales vertikales Magnetfeld zu erzeugen. Eine intrinsische Anisotropie des Mantelmaterials ist zur Erklärung der Daten nicht unbedingt erforderlich.

Die Frage nach einem Mantelplume unter dem Rheinischen Schiefergebirge kann anhand der vorliegenden Ergebnisse nicht abschließend beantwortet werden. Die Daten schließen das Vorhandensein nicht aus. Jedoch bleibt dann die Frage offen, inwieweit sich die beobachtete Richtungsabhängigkeit der elektrischen Leitfähigkeit im oberen Mantel mit der Vorstellung eines Mantelplumes vereinbaren läßt. 


\section{Zusammenfassung}

Im Rheinischen Schiefergebirge wurden in den Jahren 1997/98 umfangreiche elektromagnetische Messungen durchgeführt, deren Ergebnisse in der vorliegenden Arbeit präsentiert werden.

Insgesamt 30 Stationen deckten ein Gebiet von etwa $140 \mathrm{~km} \times 130 \mathrm{~km}$ ab. Ziel der Untersuchung war die Erstellung eines dreidimensionalen Modells der elektrischen Leitfähigkeitsverteilung unter der Eifel bis in eine Tiefe von rund $100 \mathrm{~km}$. An sämtlichen Stationen wurde sowohl das elektrische als auch das magnetische Feld aufgezeichnet, so daß die Methoden der Magnetotellurik und der erdmagnetischen Tiefensondierung gleichermaßen angewendet wurden.

Letzteres Verfahren erweist sich besonders bei der Untersuchung der Leitfähigkeitsverteilung der Erdkruste als sehr geeignet. Um die Interpretationsmöglichkeiten der magnetischen Daten zu erweitern, werden einige neue methodische Ansätze vorgeschlagen, die zum größten Teil mit Erfolg angewendet werden.

So wird unter der Westeifel eine markante Leitfähigkeitsanomalie gefunden, die sich besonders in einem starken anomalen magnetischen Feld manifestiert. Im Zentrum der Anomalie wird $\operatorname{Re}\left\{d_{D}\right\} \approx 0.4$ bei einer von Periode $256 \mathrm{~s}$ beobachtet, wobei als Referenzfeld eine Station im östlichen Bereich des Rheinischen Schiefergebirges gewählt wird. Die Daten können durch eine Leitfähigkeitsanomalie in der oberen Erdkruste $(6 \mathrm{~km}) \mathrm{mit}$ einer integrierten Leitfähigkeit von $4000 \mathrm{~S}$ und einer Ausdehnung von $150 \mathrm{~km} \times 30 \mathrm{~km}$ (Ausdehnung in Längsrichtung etwa Nord-Süd) erklärt werden.

Auch der Bereich der Hunsrück-Südrand-Störung zeigt sich deutlich als zweidimensionale Anomalie im Magnetfeld $\left(\operatorname{Re}\left\{h_{H}\right\} \approx 0.5\right.$ bei $\left.683 \mathrm{~s}\right)$. Um das Modell der Leitfähigkeitsverteilung nicht zu unübersichtlich zu gestalten, wird auf eine explizite Modellierung dieser Struktur allerdings verzichtet.

Mit den Methoden der Magnetotellurik kann unter dem gesamten Meßgebiet eine gut leitfähige Schicht in der mittleren bis unteren Kruste $(15 \mathrm{~km})$ aufgelöst werden. Sie besitzt eine anisotrope Leitfähigkeitsstruktur, die mit Hilfe eines Lamellenmodells mit den integrierten Hauptleitfähigkeiten 2000 S in Nordost-Südwestrichtung und 380 S senkrecht dazu modelliert wird.

Die Phasenkurven der Impedanz zeigen bei den meisten Stationen für lange Perioden $>1000$ s eine klare Aufspaltung beider Polarisationen. Die lokalen Induktionspfeile weisen bei einer Periode von 2731 s einheitlich in südwestliche Richtung (Realteile). Die Länge nimmt von Osten $(\approx 0.15)$ nach Westen $(\approx 0.3) \mathrm{zu}$.

Als Ursache wird eine großräumige Richtungsabhängigkeit der Leitfähigkeit im oberen 
Mantel vermutet. Die Realisierung einer anisotropen Zone in $90 \mathrm{~km}$ Tiefe mit Hilfe von gut leitfähigen Lamellen (integrierte Hauptleitfähigkeit 20.000 S in West-Ostrichtung) erklärt zwar die Phasenaufspaltung gut, nicht aber das beobachtete Verhalten der lokalen Induktionspfeile.

Auch die Modellierung mit Hilfe eines einzigen gut leitfähigen Blocks im oberen Mantel zur Erzeugung eines vertikalen Magnetfeldes gelingt nur ansatzweise. Insbesondere kann im Rahmen dieser Arbeit die Ausdehnung der Mantelanomalie nicht abgegrenzt werden. Es ist möglich, daß das einheitliche Verhalten der Induktionspfeile auf einen guten Leiter im oberen Mantel zurückzuführen ist, der zum Teil außerhalb des Rheinischen Schiefergebirges liegt.

Die Ergebnisse dieser Arbeit legen die Vorstellung eines großräumig strukturierten oberen Mantels nahe. Eine starke anisotrope Leitfähigkeit des Mantelmaterials selbst ist zur Erklärung der Daten nicht erforderlich. 


\section{Literaturverzeichnis}

Arfken, G., Mathematical Methods for Physicists, Academic Press, Inc., Orlando, 1985.

BAHR, K., Magnetotellurische Messung des elektrischen Widerstandes der Erdkruste und des oberen Mantels in Gebieten mit lokalen und regionalen Leitfähigkeitsanomalien, $\mathrm{Ph}$. D. Thesis, Institut für Geophysik, Universität Göttingen, 1985.

BAHR, K., Interpretation of the magnetotelluric impedance tensor: regional induction and local telluric distortion, J. Geophys., 62, 119-127, 1988.

BAHR, K., Geological noise in magnetotelluric data: a classification of distortion types, Phys. Earth Planet Int., 66, 24-38, 1991.

Bahr, K., Olsen, N. \& Shankland, T. J., On The Combination Of The Magnetotelluric And The Geomagnetic Depthsounding Method For Resolving An Electrical Conductivity Increase At 400 km Depth, Geophys. Res. Lett., 20, 2937-2940, 1993.

BAhr, K., \& Bruton, P., Processing of Magnetotelluric Data and their Interpretation, Geomagnetism and Earth's Interior, 4th Training School, Varanasi, 1994.

BAHR, K., Electrical anisotropy and conductivity distribution functions of fractals random networks and of the crust: the scale effect of connectivity, Geophys. J. Int., 130, 649-660, 1997.

BAI, Q. \& Kohlstedt, D. L., Substantial hydrogen solubility in olivine and implications for water storage in the mantle, Nature, 357, 672-674, 1992.

BANTIN, M., Die elektrische Leitfähigkeit der Erdkruste zwischen Weserbergland und Rheinischem Schiefergebirge - untersucht mit elektromagnetischen Methoden, Diploma Thesis, Institut für Geophysik der Universität Göttingen, 1996.

Bailey, R. C., Trapping Aqueous Fluids In The Deep Crust, Geophys. Res. Lett., 17, 1129-1132, 1990.

Baumann, H. \& Illies J. H., Stress Field and Strain Release in the Rhenish Massif, in: Fuchs, K., von Gehlen, K., Mälzer, H., Murawski, H. \& Semmel, A. (Hrsg.): Plateau Uplift. The Rhenish Shield - A Case History, Springer-Verlag, Berlin, 288-302, 1983.

Berdichevsky, M. N. \& Dmitriev, V. I., Basic Principles Of Interpretation Of Ma- 
gnetotelluric Sounding Curves, in: Ádám, A. (editor): Geoelectric and geothermal studies, Akad. Kiado, Budapest, 165-221, 1976.

Born, M, Optik, Springer, New York, 1933.

Born, M. \& Wolf, E., Principles of Optics, Pergamon, New York, 1980.

ButTKUS, B., Spektralanalyse und Filtertheorie in der angewandten Geophysik, SpringerVerlag, Berlin, 1991.

CAGNIARD, L., Basic theory of the magnetotelluric method of geophysical prospecting, Geophysics, 18, 605-635, 1953.

Chave, A. D. \& Smith, J. T., On electric and magnetic galvanic distortion tensor decompositions, J. Geophys. Res., 99:B3, 4669-4682, 1994.

Clauser, C., The Thermal Regime Of The Rhenish Massif, in: Neugebauer, H. J. (editor): Young tectonics - magmatism - fluids, a case study of the Rhenish Massif, SFB 350, Bonn, 65-67, 1998.

Constable, S., Shankland, T. J. \& Duba, A., The Electrical Conductivity of an Isotropic Olivine Mantle, J. Geophys. Res., 97:B3, 3397-3404, 1992.

DeKKer, D. L. \& HASTIE, L. M., Magneto-telluric impedances of an anisotropic layered earth model, Geophys. J. R. astr. Soc., 61, 11-20, 1980.

Duba, A. G. \& Shankland, T. J., Free Carbon \& Electrical Conductivity in The Earths Mantle, Geophys. Res. Lett., 9, 1271-1274, 1982.

Duncan, R. A., Petersen, N. \& Hargraves, R. B., Mantle Plumes, Movement of the European Plate, and Polar Wandering, Nature, 239, 82-86, 1972.

EGgERs, D. E., An Eigenstate formulation of the magnetotelluric impedance tensor, $J$. Geophys., 47, 1204-1214, 1982.

Eisbacher, G. H., Einführung in die Tektonik, Ferdinand Enke, Stuttgart, 1996.

EISEL, M. \& BAHR, K., Electrical Anisotropy in the Lower Crust of British Columbia: an Interpretation of a Magnetotelluric Profile after Tensor Decomposition, J. Geomag. Geoelectr., 45, 1115-1126, 1993.

EISEL, M., Interpretation magnetotellurischer Messungen im Umfeld der Kontinentalen Tiefbohrung unter besonderer Berücksichtigung lateral anisotroper Leitfähigkeitsstrukturen, Ph. D. Thesis, Freie Universität Berlin, 1995.

EISEL, M. \& HAAK, V., Macro-anisotropy of the electrical conductivity of the crust: a magnetotelluric study of the German Continental Deep Drilling site (KTB), Geophys. J. Int., 136, 109-122, 1999.

FILlOUX, J. H., Instrumentation and experimental methods for oceanic studies, In: Geo- 
magnetism Vol. 1, Jacobs, J. A. (editor), Academic Press, London, 1987

FluCHE, B., Erdmagnetische Tiefensondierung und Magnetotellurik in der Hessischen Senke, Diploma Thesis, Institut für Geophysik der Universität Göttingen, 1983.

Frost, B. R., FyFe, W. S., TAZAKi, K. \& CHAN, T., Grain-boundary graphite in rocks and implications for high electrical conductivity in the lower crust, Nature, 340, 134-136, 1989.

Glover, P. W. J. \& Vine, F. J., Electrical conductivity of the continental crust, Geophys. Res. Lett., 21, 2357-2360, 1994.

Gough, D. I., Seismic reflectors, conductivity, water and stress in the continental crust, Nature, 323, 143-144, 1986.

Granet, M., Wilson, M., \& Achauer, U., Imaging a mantle plume beneath the French Massif Central, Earth Planet. Sci. Lett., 136, 281-296, 1995.

Groom, R. W. \& BAiley, R. C., Decomposition of Magnetotelluric Impedance Tensors in the Presence of Local Three-Dimensional Galvanic Distortion, J. Geophys. Res., 94:B2, 1913-1925, 1989a.

Groom, R. W. \& Bailey, R. C., Some Effects Of Multiple Lateral Inhomogenities In Magnetotellurics, Geophys. Prosp., 37, 697-712, 1989 b.

Groom, R. W. \& BAILEY, R. C., Analytic investigations of the effects of near-surface three-dimensional galvanic scatterers on MT tensor decompositions, Geophysics, 56, 496$518,1991$.

Groom, R. W. \& Bahr, K., Corrections For Near Surface Effects: Decomposition Of The Magnetotelluric Impedance Tensor And Scaling Corrections For Regional Resistivities: A Tutorial, Surv. Geophys., 13, 341-379, 1992.

HAAK, V. \& Hutton, R., Electrical resistivity in continental lower crust, in: Dawson, J. B., Carswell, D. A., Hall, J. \& Wedepohl, K. H. (editors): The Nature of the Lower Continental crust, Geological Society Special Publication No. 24, Oxford, 35-49, 1986.

Habashy, T. M., Groom, R. W. \& SpIES, B. R., Beyond the Born and Rytov Approximations: A Nonlinear Approach to Electromagnetic Scattering, J. Geophys. Res., 98:B2, 1759-1775, 1993.

HAENEL, R., Geothermal Investigations in the Rhenish Massif, in: Fuchs, K., von Gehlen, K., Mälzer, H., Murawski, H. \& Semmel, A. (Hrsg.): Plateau Uplift. The Rhenish Shield A Case History, Springer-Verlag, Berlin, 288-302, 1983.

Hirsch, L. M. \& WANG, C.-Y., Electrical Conductivity of Olivine During HighTemperature Creep, J. Geophys. Res., 91:B10, 10429-10441, 1986.

HiRSCH, L. M., Enhancing mantle conductivity, Nature, 347, 232, 1990. 
HJELT, S.-E. \& KoRJA, T., Lithospheric and upper-mantle structures, results of electromagnetic soundings in Europe, Phys. Earth Planet. Int., 79, 137-177, 1993.

Hoernle, K., Zhang, Y.-S., Graham, D., Seismic and geochemical evidence for large-scale mantle upwelling beneath the eastern Atlantic and western and central Europe, Nature, 374, 34-39, 1995.

HÖNIG, M., Magnetotellurik im Rheinischen Schiefergebirge unter Berücksichtigung von Oberflächenverzerrungen und dem Einfluß der anisotropen mittleren Kruste, Diploma Thesis, Institut für Geophysik der Universität Göttingen, 1998.

Hyndman, R. D. \& Shearer, P. M., Water in the lower continental crust: modelling magnetotelluric and seismic reflection results, Geophys. J. Int., 98, 343-365, 1989.

IRVING, E., Drift of the major continental blocks since the Devonian, Nature, 270, 304309, 1977.

Jakoby, W. R., Joachimi, H. \& Gerstenecker, C., The Gravity Field of the Rhenish Massif, in: Fuchs, K., von Gehlen, K., Mälzer, H., Murawski, H. \& Semmel, A. (Hrsg.): Plateau Uplift. The Rhenish Shield - A Case History, Springer-Verlag, Berlin, 288-302, 1983.

Jantos, C., Audiomagnetotellurische Messungen mit der neuen Göttinger „Midband“ Anlage, Diploma Thesis, Institut für Geophysik der Universität Göttingen, 1998.

Jenkins, G. M. \& Watts, D. G., Spectral Analysis and its Applications, Holden Day, San Francisco, 1968.

Jödicke, H., Untiedt, J., Olgemann, W., Schulte, L. \& Wagenitz, V., Electrical Conductivity Structure of the Crust and Upper Mantle Beneath the Rhenish Massif, in: Fuchs, K., von Gehlen, K., Mälzer, H., Murawski, H. \& Semmel, A. (Hrsg.): Plateau Uplift. The Rhenish Shield - A Case History, Springer-Verlag, Berlin, 288-302, 1983.

Jones, A. G., Electrical conductivity of the continental lower crust, in: Fountain, D. M., Arculus, R. \& Kay, R. W. (editors): Continental Lower Crust, Developments in Geotectonics No. 23, Elsevier, Amsterdam, 81-143, 1992.

Jones, A. G., Groom, R. W. \& Kurtz. R. D., Decomposition and Modelling of the BC87 Dataset, J. Geomag. Geoelectr., 45, 1127-1150, 1993.

Jones, A. G. \& Groom, R. W., Strike-angle determination from the magnetotelluric impedance tensor in the presence of noise and local distortion: rotate at your peril!, Geophys. J. Int., 113, 524-534, 1993.

Junge, A., A new telluric $\mathrm{KCl}$ probe using Filloux's $\mathrm{Ag}-\mathrm{AgCl}$ Electrode. PAGEOPH, 134, 589-598, 1990.

JUNGE, A., Induzierte erdelektrische Felder - neue Beobachtungen in Norddeutschland 
und im Bramwald, Habil. Math. Nat. Fak., Göttingen, 1994.

KARATO, S., The role of hydrogen in the electrical conductivity of the upper mantle, Nature, 347, 272-273, 1990.

Kellett, R. L., Mareschal, M. \& Kurtz, R. D., A model of lower crustal electrical anisotropy for the Pontiac Subprovince of the Canadian Shield, Geophys. J. Int., 111, 141150, 1992.

Kemmerle, K., Magnetotellurik am Alpen-Nordrand mit Diskussion der lokalen Effekte und Darstellung einer Einzeleffekt-Auswertung, Ph. D. Thesis, Fachbereich Geowissenschaften, München, 1977.

KISSIN, I. G., Fluid Saturation, Electrical Conductivity, and Seismicity of the Crust, Izvestiya, Physics of the Solid Earth, 32, 289-298, 1996.

Kong, J. A., Theory Of Electromagnetic Waves, John Wiley \& Sons, New York, 1975.

KURAS, O., Elektromagnetische Arraymessungen im westlichen Rheinischen Schiefergebirge: Simulation des Induktionsprozesses mit optimierten dreidimensionalen Modellen, Diploma Thesis, Institut für Geophysik der Universität Göttingen, 1998.

Kurtz, R. D., Craven, J. A., Niblett, E. R. \& Stevens, R. A., The conductivity of the crust and mantle beneath the Kapuskasing Uplift: electrical anisotropy in the upper mantle, Geophys. J. Int., 113, 483-498, 1993.

LARSEN, J. C., Removal of local surface conductivity effects from low frequency mantle response curves, Acta Geodaet., Geophys. et Montanist. Acad. Sci. Hung., 12, 183-186, 1977.

LEIBECKER, J. \& KuRAS, O., Elektromagnetische Array-Messungen im westlichen Rheinischen Schiefergebirge: Interpretation und Modellierung geomagnetischer Übertragungsfunktionen, ETS Kolloquiumsband, Neustadt a. d. Weinstraße, 1998.

Lippolt, H. J., Distribution of Volcanic Activity in Space and Time, in: Fuchs, K., von Gehlen, K., Mälzer, H., Murawski, H. \& Semmel, A. (Hrsg.): Plateau Uplift. The Rhenish Shield - A Case History, Springer-Verlag, Berlin, 113-119, 1983.

Lizarralde, D., Chave, A., Hirth, G. \& Schultz, A., Northeastern Pacific mantle conductivity profile from long-period magnetotelluric sounding using Hawaii-to-California submarine cable data, J. Geophys. Res., 100:B9, 17837-17854, 1995.

Mackie, R. L., Madden, T. R. \& Wannamaker, P. E., Three-dimensional magnetotelluric modeling using difference equations - Theory and comparisons to integral equation solutions, Geophysics, 58, 215-226, 1993.

MACKIE, R. L., \& MAdDEN, T. R., Conjugate direction relaxation solutions for 3-D magnetotellturic modeling, Geophysics, 58, 1053-1057, 1993. 
Mackie, R. L., Smith, J. T. \& Madden, T. R., Three-dimensional electromagnetic modeling using finite difference equations: The magnetotelluric example, Radio Science, 29, 923-935, 1994.

Mackwell, S. J. \& Kohlstedt, D. L., Diffusion of Hydrogen in Olivine: Implications for Water in the Mantle, J. Geophys. Res., 95:B4, 5079-5088, 1990.

MÄLZER, H., Hein, G. \& Zippelt, K., Height Changes in the Rhenish Massif: Determination and Analysis, in: Fuchs, K., von Gehlen, K., Mälzer, H., Murawski, H. \& Semmel, A. (Hrsg.): Plateau Uplift. The Rhenish Shield - A Case History, Springer-Verlag, Berlin, 113-119, 1983.

Mareschal, M., Fyfe, W. S., Percival, J. \& Chan, T., Grain-boundary graphite in Kapuskasing gneisses and implications for lower-crustal conductivity, Nature, 357, 674676, 1992.

MARKL, G. \& BUChER, K., Composition of fluids in the lower crust inferred from metamorphic salt in lower crustal rocks, Nature, 391, 781-782, 1998.

MARQuis, G., \& HYNDMAn, R. D., Geophysical support for aqueous fluids in the deep crust: seismic and electrical relationships, Geophys. J. Int., 110, 91-105, 1992.

MAURER, H.-M., Elektromagnetische Induktion in anisotropen Leitern, Ph. D. Thesis, Naturwissenschaftliche Fakultät, Technische Universität Braunschweig , 1993.

Meyer, W. et al., Pre-Quaternary Uplift in the Central Part of the Rhenish Massif, in: Fuchs, K., von Gehlen, K., Mälzer, H., Murawski, H. \& Semmel, A. (Hrsg.): Plateau Uplift. The Rhenish Shield - A Case History, Springer-Verlag, Berlin, 9-38, 1983.

Meyer, W., Geologie der Eifel, 3. Aufl., E. Schweizbart'sche Verlagsbuchhandlung, Stuttgart, 1994.

Meyer, W. \& Stets, J., Young Pleistocene To Recent Uplift In The Rhenish Massif, in: Neugebauer, H. J. (editor): Young tectonics - magmatism - fluids, a case study of the Rhenish Massif, SFB 350, Bonn, 7-11, 1998a.

Meyer, W. \& Stets, J., Junge Tektonik im Rheinischen Schiefergebirge und ihre Quantifizierung, Z. dt. geol. Ges., 359-379, 1998b.

Mibe, K. FujiI, T. \& YAsudA, A., Connectivity of aqueous fluid in the Earth's upper mantle, Geophys. Res. Lett., 25, 1233-1236, 1998.

Morgan, W. J., Plate motions and deep mantle convection, Mem. Geol. Soc, Am, 132, $7-22,1972$.

MurAwsKi, H. et al., Regional Tectonic Setting and Geological Structure of the Rhenish Massif, in: Fuchs, K., von Gehlen, K., Mälzer, H., Murawski, H. \& Semmel, A. (Hrsg.): Plateau Uplift. The Rhenish Shield - A Case History, Springer-Verlag, Berlin, 9-38, 1983. 
MurawsKi, H., Geologisches Wörterbuch, Ferdinand Enke Verlag, Stuttgart, 1992.

Neugebauer, H. J., Woidt, W.-D. \& Wallner, H., Uplift, Volcanism and Tectonics: Evidence for Mantle Diapirs at the Rhenish Massif, in: Fuchs, K., von Gehlen, K., Mälzer, H., Murawski, H. \& Semmel, A. (Hrsg.): Plateau Uplift. The Rhenish Shield - A Case History, Springer-Verlag, Berlin, 381-403, 1983.

Oettinger, G., Neubearbeitung und Ergänzung eines Magnetotellurik-Profils in der Eifel, ETS Kolloquiumsband, Hoechst, 1994.

Olsen, N., The electrical conductivity of the mantle beneath Europe derived from Cresponses from 3 to $720 \mathrm{hr}$, Geophys. J. Int, 133, 298-308, 1998.

PARKInSON, W. D., Direction of rapid geomagnetic fluctuations, Geophys. J., 2, 1-14, 1959.

Peter, C., Elektrische Leitfähigkeit der Erdkruste in Südhannover, Nordhessen und im westlichen Thüringen, abgeleitet aus der elektromagnetischen Induktion durch natürliche Felder, $P h$. D. Thesis, Institut für Geophysik, Universität Göttingen, 1994.

Praus, O., PĚčová, J., Petr. V., Babuška, V., \& Plomerová, J., Magnetotelluric and seismological determination of the lithosphere-asthenosphere transition in Central Europe, Phys. Earth Planet Int., 60, 212-228, 1990.

Press, F. \& SiEVER, R., Allgemeine Geologie, Spektrum Akademischer Verlag, Heidelberg, 1995.

Prodehl, C., Mueller, S. \& HaAk, V., The European Cenozoic Rift System, in: Olsen, K. H. (Editor): Continental Rifts: Evolution, Structure, Tectonics, Elsevier, Amsterdam, 133-212, 1995.

RAikes, S. \& Bonjer, K.-P., Large Scale Mantle Heterogenity Beneath the Rhenish Massif and Its Vicinity from Teleseismic P-Residuals Measurements, in: Fuchs, K., von Gehlen, K., Mälzer, H., Murawski, H. \& Semmel, A. (Hrsg.): Plateau Uplift. The Rhenish Shield - A Case History, Springer-Verlag, Berlin, 315-331, 1983.

Ritter, J. R. R., Achauer, U., Christensen, U. R. \& The Eifel Plume Team, The Teleseismic Tomography Experiment in the Eifel Region, Central Europe: Design and First Results, submitted to Seism. Res. Lett., 2000.

RitTer, P., Separation of Local and Regional Information in Geomagnetic Response Functions using Hypothetical Event Analys, Ph. D. Thesis, Dept. of Geology and Geophysics, Univ. of Edinburgh, 1996.

Roberts, J. J. \& Tyburczy, J. A., Partial-melt electrical conductivity: Influence of melt compositon, J. Geophys. Res., 104:B4, 7055-7065, 1999.

SATO, H. \& IDA, Y., Low frequency electrical impedance of partially molten Gabbro: 
The effect of melt geometry on electrical properties, Tectonophysics, 107, 105-134, 1984.

SCHEELKE, I, Magnetotellurische Messungen im Rheingraben und ihre Deutung mit zweidimensionalen Modellen, Gamma, 20, 1972.

Schmeling, H., Numerical models on the influence of partial melt on elastic, anelastic and electrical properties of rocks. Part II: electrical conductivity, Phys. Earth Planet. Int., 43, 123-136, 1986.

Schmincke, H.-U., LoRenz, V. \& SECK, H. A., The Quaternary Eifel Volcanic Fields, in: Fuchs, K., von Gehlen, K., Mälzer, H., Murawski, H. \& Semmel, A. (Hrsg.): Plateau Uplift. The Rhenish Shield - A Case History, Springer-Verlag, Berlin, 139-151, 1983.

SCHMINCKe, H.-U., Vulkanismus, Wissenschaftl. Buchgesellschaft, Darmstadt, 1986.

SchmucKer, U., Anomalies Of Geomagnetic Variations In The Southwestern United States, Bull. Scripps Institution of Oceanography, 13, 1970.

SCHMUCKER, U., Direkte und iterative Verfahren zur Behandlung 2-dimensionaler Leitfähigkeitsmodelle, ETS Kolloquiumsband, Grafrath, 1974.

SChMUCKER, U. \& WeIdelt, P., Electromagnetic Induction In The Earth, Lecture Notes Aarhus, 1975.

SCHMUCKER, U., Magnetic field of the earth, Landolt-Börnstein, Geophysics of the Solid Earth, the Moon and the planets, 2b, 1984.

SCHMUCKER, U., Substitute Conductors for Electromagnetic Response Estimates, PAGEOPH, 125, 341-367, 1987.

SCHMUCKer, U., 2D Modelling with Linearized Integral Equations, J. Geomag. Geoelectr., 45, 1045-1062, 1993.

SCHNEIDER, E., Dreidimensionale Modellrechnungen für elektromagnetische Daten eines Profils über die Göttinger D-Anomalie, Diploma Thesis, Institut für Geophysik der Universität Göttingen, 1998.

SIEMON, B., Ein Interpretationsverfahren für induktiv schwach gekoppelte Leitfähigkeitsanomalien, dargestellt am Beispiel des Salzstockes Wesendorf im Gifhorner Trog, Ph. D. Thesis, Institut für Geophysik, Universität Göttingen, 1991.

SiEmon, B., An interpretation technique for superimposed induction anomalies, Geophys. J. Int., 130, 73-88, 1997.

Simpson, F. \& WARner, M., Coincident magnetotelluric, P-wave and S-wave images of the deep continental crust beneath the Weardale granite, NE England: seismic layering, low conductance and implications against the fluids paradigm, Geophys. J. Int., 133, 419434, 1998. 
Smith, J. T., Estimating galvanic-distortion magnetic fields in magnetotellurics, Geophys. J. Int., 130, 65-72, 1997.

SOYER, W., Elekromagnetische Arraymessungen im westlichen Rheinischen Schiefergebirge: Auswertung langer Perioden mit dem Z:H-Verfahren, Diploma Thesis, Institut für Geophysik der Universität Göttingen, 1998.

Steveling, E. \& LeVen, M., Ein Datenlogger für niederfrequente geophysikalische Messungen, ETS Kolloquiumsband, Borkheide, 1992.

SwIFT, C. M., A Magnetotelluric Investigation Of An Electrical Conductivity Anomaly In The Southwestern United States, Project Report, M. I. T., 1967.

Szarka, L. \& Menvielle, M., Analysis of rotational invariants of the magnetotelluric impedance tensor, Geophys. J. Int., 129, 133-142, 1997.

Ting, S. C. \& Hohmann, G. W., Integral equation modeling of three-dimensional magnetotelluric response, Geophysics, 46, 182-197, 1981.

VAN'YAN, L. L. \& HyNDMAN, R. D., On the Origin of Electrical Conductivity in the Consolidated Crust, Izvestiya, Physics of the Solid Earth, 32, 266-271, 1996.

VAN'YAN, L. L. \& GLIKO, A. O., Seismic and electromagnetic evidence of dehydration as a free water source in the reactivated crust, 137, 159-162, 1999.

Volbers, R., JÖDICKE, H. \& UNTIEDT, J., Magnetotelluric study of the earth's crust along the deep seismic reflection profile DEKORP 2-N, Geologische Rundschau, 79/3, 581-601, 1990.

WAIT, J. R., Propagation of radio waves over a stratified ground, Geophysics, 18, 416422, 1953.

Walter, R., Geologie von Mitteleuropa, 6. Aufl., E. Schweizbart'sche Verlagsbuchhandlung, Stuttgart, 1995.

WATERMAnN, J., Beobachtung korrelierter ULF-Fluktuation im erdmagnetischen Feld und im Phasenweg ionosphärischer HF-Sondierungen, $P h$. D. Thesis, Institut für Geophysik, Universität Göttingen, 1984.

WeAver, J. T., Mathematical Methods for Geo-electromagnetic Induction, Research Studies Press Ltd., New York, 1994.

WeIDELT, P., The Inverse Problem of Geomagnetic Induction, Z.f. Geophysik, 38, 257289, 1972.

WEIDELT, P., Phasenbeziehungen für die B-Polarisation, ETS Kolloquiumsband, Höchst im Odenwald, 1994.

WiEsE, H., Geomagnetische Tiefentellurik II: Die Streichrichtung der Untergrundstruk- 
turen des elektrischen Widerstandes, erschlossen aus geomagnetischen Variationen, Geofisica pura e applicata, 52, 83-103, 1962.

Wilson, J. T., A possible origin of the Hawaiian islands, Can. J. Phys., 41, 863-870, 1963.

Wilson, M. \& Downes, H., Mafic alkaline magmatism associated with the European Cenozoic rift system, Tectonophysics, 208, 173-182, 1992.

Xu, Y., Poe, B. T., Shankland, T. J. \& Rubie, D. C., Electrical Conductivity of Olivine, Wadsleyite, and Ringwoodite Under Upper-Mantle Conditions, Science, 280, 14151418, 1998.

Yardley, B. W. D. \& Valley, J. W., The petrologic case for a dry lower crust, $J$. Geophys. Res, 102:B6, 12173-12185, 1997.

Yee, E. \& Paulson, K. V., The canonical decomposition and its relationship to other forms of the magnetotelluric impedance tensor analysis, J. Geophys., 61, 173-189, 1987.

Zhamaletdinov, A. A., Graphite in the Earth's Crust and Electrical Conductivity Anomalies, Izvestiya, Physics of the Solid Earth, 32, 272-288, 1996.

Zhang, P., Roberts, R. G. \& Pedersen, L. B., Magnetotelluric strike rules, Geophysics, 52, 267-278, 1987.

Zhang, P., Pedersen, L. B., Mareschal, M. \& Chouteau, M., Channeling contribution to tipper vectors: a magnetic equivalent to electrical distortion, Geophys. J. Int., 113, 693-700, 1993. 


\section{A Anhang}

\section{A.1 Stationskoordinaten}

\begin{tabular}{|l|l|l||l|l|l|}
\hline Station & Östl. L. $\left[{ }^{\circ}\right.$ ] & Nördl. B. $\left[{ }^{\circ}\right]$ & Station & Östl. L. $\left[{ }^{\circ}\right]$ & Nördl. B. $\left[{ }^{\circ}\right.$ ] \\
\hline \hline AHR & 7.13008 & 50.52532 & HIL & 6.65483 & 50.31136 \\
\hline ALT & 7.53339 & 50.73582 & HON & 6.80169 & 50.42097 \\
\hline BAD & 6.80689 & 50.56394 & HUR & 6.34412 & 50.51509 \\
\hline BIT & 6.58687 & 50.09800 & IDA & 7.35369 & 49.68488 \\
\hline BKR & 7.87532 & 49.79088 & IRR & 6.37063 & 49.87916 \\
\hline BON & 6.97050 & 50.71219 & LAH & 7.73146 & 50.30146 \\
\hline BOO & 7.01430 & 50.29030 & MAY & 7.41792 & 50.34608 \\
\hline CHA & 8.19551 & 50.13346 & NEU & 6.18116 & 50.09596 \\
\hline COC & 7.26308 & 50.13359 & OSB & 6.77756 & 49.66874 \\
\hline DAH & 6.43897 & 50.39814 & PRU & 6.38231 & 50.28650 \\
\hline DAU & 6.92781 & 50.10211 & SAA & 6.93111 & 49.44373 \\
\hline DID & 7.48746 & 50.55034 & SEL & 7.80044 & 50.53312 \\
\hline DIE & 8.04942 & 50.29019 & TRA & 7.07843 & 49.86483 \\
\hline FOE & 6.72380 & 49.86679 & WAL & 6.81325 & 50.23078 \\
\hline GOA & 7.64850 & 50.06166 & & & \\
\hline
\end{tabular}

Tabelle A.1: Koordinaten der Feldstationen. 


\section{A.2 Elemente des Perturbationstensors nach Wechsel der Bezugsstation}

Gegeben seien zwei Perturbationstensoren der Stationen $A$ und $C$ bezogen auf eine Referenzstation $B: \underline{\mathbf{W}}^{A, B}$ und $\underline{\mathbf{W}}^{C, B}$.

Nach einem Wechsel der Referenzstation nach Gleichung (2.23) lauten die Elemente des Perturbationstensors $\underline{\mathbf{W}}^{A, C}$ der Station $A$ bezogen auf die neue Referenz $C$ in expliziter Form:

$$
\begin{aligned}
h_{H}^{A, C} & =\frac{\left(h_{H}^{A, B}-h_{H}^{C, B}\right)\left(1+d_{D}^{C, B}\right)-\left(h_{D}^{A, B}-h_{D}^{C, B}\right) d_{H}^{C, B}}{\left(1+h_{H}^{C, B}\right)\left(1+d_{D}^{C, B}\right)-h_{D}^{C, B} d_{H}^{C, B}} \\
h_{D}^{A, C} & =\frac{\left(h_{D}^{A, B}-h_{D}^{C, B}\right)\left(1+h_{H}^{C, B}\right)-\left(h_{H}^{A, B}-h_{H}^{C, B}\right) h_{D}^{C, B}}{\left(1+h_{H}^{C, B}\right)\left(1+d_{D}^{C, B}\right)-h_{D}^{C, B} d_{H}^{C, B}} \\
d_{H}^{A, C} & =\frac{\left(d_{H}^{A, B}-d_{H}^{C, B}\right)\left(1+d_{D}^{C, B}\right)-\left(d_{D}^{A, B}-d_{D}^{C, B}\right) d_{H}^{C, B}}{\left(1+h_{H}^{C, B}\right)\left(1+d_{D}^{C, B}\right)-h_{D}^{C, B} d_{H}^{C, B}} \\
d_{D}^{A, C}= & \frac{\left(d_{D}^{A, B}-d_{D}^{C, B}\right)\left(1+h_{H}^{C, B}\right)-\left(d_{H}^{A, B}-d_{H}^{C, B}\right) h_{D}^{C, B}}{\left(1+h_{H}^{C, B}\right)\left(1+d_{D}^{C, B}\right)-h_{D}^{C, B} d_{H}^{C, B}} .
\end{aligned}
$$

\section{A.3 Nachträge zum Prozessing}

\section{A.3.1 Gewichtsfunktionen}

- Hanning-Fenster

$$
w(t)=\left\{\begin{array}{lll}
\frac{1}{2}\left(1+\cos \left(\frac{\pi t}{T}\right)\right) & \text { für } & |t| \leq \frac{T}{2} \\
0 & \text { für } & |t|>\frac{T}{2}
\end{array}\right.
$$

- Parzen-Fenster

$$
P_{i}=P_{0}\left(\frac{\sin \left(i \pi / N_{p}\right)}{i \pi / N_{p}}\right)^{4}
$$

mit

$$
N_{p}=1+\operatorname{Int}\left(\frac{f_{e} N}{2 \Delta f}\right) \quad \text { und } \quad P_{0}=\frac{1.5}{N_{p}} .
$$




\section{A.3.2 Explizite Ausdrücke für alle Übertragungsfunktionen}

- Magnetotellurik

Die Elemente des Impedanztensors inklusive der Übertragungsfunktionen des vertikalen Magnetfeldes lauten:

$$
\begin{aligned}
& Z_{x x}=\left(\left\langle E_{x} B_{x}^{*}\right\rangle\left\langle B_{y} B_{y}^{*}\right\rangle-\left\langle E_{x} B_{y}^{*}\right\rangle\left\langle B_{y} B_{x}^{*}\right\rangle\right) / D \\
& Z_{x y}=\left(\left\langle E_{x} B_{y}^{*}\right\rangle\left\langle B_{x} B_{x}^{*}\right\rangle-\left\langle E_{x} B_{x}^{*}\right\rangle\left\langle B_{x} B_{y}^{*}\right\rangle\right) / D \\
& Z_{y x}=\left(\left\langle E_{y} B_{x}^{*}\right\rangle\left\langle B_{y} B_{y}^{*}\right\rangle-\left\langle E_{y} B_{y}^{*}\right\rangle\left\langle B_{y} B_{x}^{*}\right\rangle\right) / D \\
& Z_{y y}=\left(\left\langle E_{y} B_{y}^{*}\right\rangle\left\langle B_{x} B_{x}^{*}\right\rangle-\left\langle E_{y} B_{x}^{*}\right\rangle\left\langle B_{x} B_{y}^{*}\right\rangle\right) / D \\
& Z_{z x}=\left(\left\langle B_{z} B_{x}^{*}\right\rangle\left\langle B_{y} B_{y}^{*}\right\rangle-\left\langle B_{z} B_{y}^{*}\right\rangle\left\langle B_{y} B_{x}^{*}\right\rangle\right) / D \\
& Z_{z y}=\left(\left\langle B_{z} B_{y}^{*}\right\rangle\left\langle B_{x} B_{x}^{*}\right\rangle-\left\langle B_{z} B_{x}^{*}\right\rangle\left\langle B_{x} B_{y}^{*}\right\rangle\right) / D .
\end{aligned}
$$

Die Vertrauensbereiche der Übertragungsfunktionen werden nach WATERMANN (1984) berechnet:

$$
\begin{aligned}
\Delta Z_{x x} & =\sqrt{f_{2}(\mathrm{v})\left\langle E_{x} E_{x}^{*}\right\rangle\left(1-R_{x}\right) /\left(\left\langle B_{x} B_{x}^{*}\right\rangle(1-U)\right)} \\
\Delta Z_{x y} & =\sqrt{f_{2}(\mathrm{v})\left\langle E_{x} E_{x}^{*}\right\rangle\left(1-R_{x}\right) /\left(\left\langle B_{y} B_{y}^{*}\right\rangle(1-U)\right)} \\
\Delta Z_{y x} & =\sqrt{f_{2}(\mathrm{v})\left\langle E_{y} E_{y}^{*}\right\rangle\left(1-R_{y}\right) /\left(\left\langle B_{x} B_{x}^{*}\right\rangle(1-U)\right)} \\
\Delta Z_{y y} & =\sqrt{f_{2}(\mathrm{v})\left\langle E_{y} E_{y}^{*}\right\rangle\left(1-R_{y}\right) /\left(\left\langle B_{y} B_{y}^{*}\right\rangle(1-U)\right)} \\
\Delta Z_{z x} & =\sqrt{f_{2}(\mathrm{v})\left\langle B_{z} B_{z}^{*}\right\rangle\left(1-R_{z}\right) /\left(\left\langle B_{x} B_{x}^{*}\right\rangle(1-U)\right)} \\
\Delta Z_{z y} & =\sqrt{f_{2}(\mathrm{v})\left\langle B_{z} B_{z}^{*}\right\rangle\left(1-R_{z}\right) /\left(\left\langle B_{y} B_{y}^{*}\right\rangle(1-U)\right)}
\end{aligned}
$$

wobei

$$
D=\left(\left\langle B_{x} B_{x}^{*}\right\rangle\left\langle B_{y} B_{y}^{*}\right\rangle-\left\langle B_{x} B_{y}^{*}\right\rangle\left\langle B_{y} B_{x}^{*}\right\rangle\right) .
$$

$R_{i}$ bezeichnet die quadratische bivariate Kohärenz und $U$ die quadratische univariate Kohärenz:

$$
\begin{aligned}
R_{x} & =\left(Z_{x x}\left\langle B_{x} E_{x}^{*}\right\rangle+Z_{x y}\left\langle B_{y} E_{x}^{*}\right\rangle\right) /\left\langle E_{x} E_{x}^{*}\right\rangle \\
R_{y} & =\left(Z_{y x}\left\langle B_{x} E_{y}^{*}\right\rangle+Z_{y y}\left\langle B_{y} E_{y}^{*}\right\rangle\right) /\left\langle E_{y} E_{y}^{*}\right\rangle \\
R_{z} & =\left(Z_{z x}\left\langle B_{x} B_{z}^{*}\right\rangle+Z_{z y}\left\langle B_{y} B_{z}^{*}\right\rangle\right) /\left\langle B_{z} B_{z}^{*}\right\rangle \\
U & =\left\langle B_{x} B_{y}^{*}\right\rangle\left\langle B_{y} B_{x}^{*}\right\rangle /\left(\left\langle B_{x} B_{x}^{*}\right\rangle\left\langle B_{y} B_{y}^{*}\right\rangle\right) .
\end{aligned}
$$

Außerdem ist

$$
f_{2}(v)=\frac{4}{4-v} F_{4, v-4}(\beta)
$$

wobei $F_{4, v-4}(\beta)$ die Fisher-Verteilung mit $\beta=68 \%$ bezeichnet. $v$ gibt die Anzahl der Freiheitsgrade an. 
- Erdmagnetische Tiefensondierung

Die Elemente des Perturbationstensors inklusive der Übertragungsfunktionen des vertikalen Magnetfeldes lauten:

$$
\begin{aligned}
h_{H} & =\left(\left\langle B_{x} B_{x}^{n *}\right\rangle\left\langle B_{y}^{n} B_{y}^{n *}\right\rangle-\left\langle B_{x} B_{y}^{n *}\right\rangle\left\langle B_{y}^{n} B_{x}^{n *}\right\rangle\right) / D \\
h_{D} & =\left(\left\langle B_{x} B_{y}^{n *}\right\rangle\left\langle B_{x}^{n} B_{x}^{n *}\right\rangle-\left\langle B_{x} B_{x}^{n *}\right\rangle\left\langle B_{x}^{n} B_{y}^{n *}\right\rangle\right) / D \\
d_{H} & =\left(\left\langle B_{y} B_{x}^{n *}\right\rangle\left\langle B_{y}^{n} B_{y}^{n *}\right\rangle-\left\langle B_{y} B_{y}^{n *}\right\rangle\left\langle B_{y}^{n} B_{x}^{n *}\right\rangle\right) / D \\
d_{D} & =\left(\left\langle B_{y} B_{y}^{n *}\right\rangle\left\langle B_{x}^{n} B_{x}^{n *}\right\rangle-\left\langle B_{y} B_{x}^{n *}\right\rangle\left\langle B_{x}^{n} B_{y}^{n *}\right\rangle\right) / D \\
z_{H} & =\left(\left\langle B_{z} B_{x}^{n *}\right\rangle\left\langle B_{y}^{n} B_{y}^{n *}\right\rangle-\left\langle B_{z} B_{y}^{n *}\right\rangle\left\langle B_{y}^{n} B_{x}^{n *}\right\rangle\right) / D \\
z_{D} & =\left(\left\langle B_{z} B_{y}^{n *}\right\rangle\left\langle B_{x}^{n} B_{x}^{n *}\right\rangle-\left\langle B_{z} B_{x}^{n *}\right\rangle\left\langle B_{x}^{n} B_{y}^{n *}\right\rangle\right) / D \\
\Delta h_{H} & =\sqrt{f_{2}(\mathrm{v})\left\langle B_{x} B_{x}^{*}\right\rangle\left(1-R_{x}\right) /\left(\left\langle B_{x}^{n} B_{x}^{n *}\right\rangle(1-U)\right)} \\
\Delta h_{D} & =\sqrt{f_{2}(\mathrm{v})\left\langle B_{x} B_{x}^{*}\right\rangle\left(1-R_{x}\right) /\left(\left\langle B_{y}^{n} B_{y}^{n *}\right\rangle(1-U)\right)} \\
\Delta d_{H} & =\sqrt{f_{2}(\mathrm{v})\left\langle B_{y} B_{y}^{*}\right\rangle\left(1-R_{y}\right) /\left(\left\langle B_{x}^{n} B_{x}^{n *}\right\rangle(1-U)\right)} \\
\Delta d_{D} & =\sqrt{f_{2}(\mathrm{v})\left\langle B_{y} B_{y}^{*}\right\rangle\left(1-R_{y}\right) /\left(\left\langle B_{y}^{n} B_{y}^{n *}\right\rangle(1-U)\right)} \\
\Delta z_{H} & =\sqrt{f_{2}(\mathrm{v})\left\langle B_{z} B_{z}^{*}\right\rangle\left(1-R_{z}\right) /\left(\left\langle B_{x}^{n} B_{x}^{n *}\right\rangle(1-U)\right)} \\
\Delta z_{D} & =\sqrt{f_{2}(\mathrm{v})\left\langle B_{z} B_{z}^{*}\right\rangle\left(1-R_{z}\right) /\left(\left\langle B_{y}^{n} B_{y}^{n *}\right\rangle(1-U)\right)}
\end{aligned}
$$

wobei

$$
\begin{aligned}
D & =\left(\left\langle B_{x}^{n} B_{x}^{n *}\right\rangle\left\langle B_{y}^{n} B_{y}^{n *}\right\rangle-\left\langle B_{x}^{n} B_{y}^{n *}\right\rangle\left\langle B_{y}^{n} B_{x}^{n *}\right\rangle\right) \\
R_{x} & =\left(h_{H}\left\langle B_{x}^{n} B_{x}^{*}\right\rangle+h_{D}\left\langle B_{y}^{n} B_{x}^{*}\right\rangle\right) /\left\langle B_{x} B_{x}^{*}\right\rangle \\
R_{y} & =\left(d_{H}\left\langle B_{x}^{n} B_{y}^{*}\right\rangle+d_{D}\left\langle B_{y}^{n} B_{y}^{*}\right\rangle\right) /\left\langle B_{y} B_{y}^{*}\right\rangle \\
R_{z} & =\left(z_{H}\left\langle B_{x}^{n} B_{z}^{*}\right\rangle+z_{D}\left\langle B_{y}^{n} B_{z}^{*}\right\rangle\right) /\left\langle B_{z} B_{z}^{*}\right\rangle \\
U & =\left\langle B_{x}^{n} B_{y}^{n *}\right\rangle\left\langle B_{y}^{n} B_{x}^{n *}\right\rangle /\left(\left\langle B_{x}^{n} B_{x}^{n *}\right\rangle\left\langle B_{y}^{n} B_{y}^{n *}\right\rangle\right) .
\end{aligned}
$$

\section{A.3.3 Gewichtete Stapelung der geglätteten Spektren}

Zur Qualitätsbewertung der geglätteten Spektren wird die Größe

$$
w=\left(\frac{R_{x}+R_{y}}{2}\right)^{N}
$$

verwendet. Als Standardexponent wird $N=2$ gesetzt. Falls $w<0.3$ ist, werden die entsprechenden Spektren ausgesondert, anderenfalls mit dem Gewicht $w$ gestapelt. 


\section{A.4 Göttinger Datenformate}

\section{A.4.1 *.mtt-Format}

Für jede Meßstation werden für eine gewisse Anzahl Frequenzen Übertragungsfunktionen berechnet, die einem linearem Ansatz

$$
\begin{aligned}
E_{x} & =Z_{x x} B_{x}+Z_{x y} B_{y} \\
E_{y} & =Z_{y x} B_{x}+Z_{y y} B_{y} \\
B_{z} & =Z_{z x} B_{x}+Z_{z y} B_{y}
\end{aligned}
$$

genügen. Sie werden als ASCII-Dateien in folgendem Format gespeichert. Die Frequenz wird in $\mathrm{Hz}$ angegeben, die Elemente des Impedanztensors besitzen die Einheit km/s.

$\begin{array}{llllllll}\text { Frequenz } & \text { Freiheitsgrade } & & & & \\ \operatorname{Re}\left\{Z_{x x}\right\} & \operatorname{Im}\left\{Z_{x x}\right\} & \operatorname{Re}\left\{Z_{x y}\right\} & \operatorname{Im}\left\{Z_{x y}\right\} & \operatorname{Re}\left\{Z_{y x}\right\} & \operatorname{Im}\left\{Z_{y x}\right\} & \operatorname{Re}\left\{Z_{y y}\right\} & \operatorname{Im}\left\{Z_{y y}\right\} \\ \Delta Z_{x x} & \Delta Z_{x y} & \Delta Z_{y x} & \Delta Z_{y y} & \operatorname{Re}\left\{Z_{z x}\right\} & \operatorname{Im}\left\{Z_{z x}\right\} & \operatorname{Re}\left\{Z_{z y}\right\} & \operatorname{Im}\left\{Z_{z y}\right\} \\ \Delta Z_{z x} & \Delta Z_{z y} & R_{x} & R_{y} & R_{z} & & & \end{array}$

\section{A.4.2 *.gdt-Format}

Der lineare Zusammenhang zwischen dem anomalen magnetischen Feld an der Meßstation und dem normalen Feld einer Bezugsstation lautet:

$$
\begin{aligned}
B_{x}^{a} & =h_{H} B_{x}^{n}+h_{D} B_{y}^{n} \\
B_{y}^{a} & =d_{H} B_{x}^{n}+d_{D} B_{y}^{n} \\
B_{z}^{a} & =z_{H} B_{x}^{n}+z_{D} B_{y}^{n} .
\end{aligned}
$$

Das Format ist im übrigen analog zu dem *.mtt-Format aufgebaut. Die magnetischen Übertragungsfunktionen sind dimensionslos.

$\begin{array}{llllllll}\text { Frequenz Freiheitsgrade } & & & & \\ \operatorname{Re}\left\{h_{H}\right\} & \operatorname{Im}\left\{h_{H}\right\} & \operatorname{Re}\left\{h_{D}\right\} & \operatorname{Im}\left\{h_{D}\right\} & \operatorname{Re}\left\{d_{H}\right\} & \operatorname{Im}\left\{d_{H}\right\} & \operatorname{Re}\left\{d_{D}\right\} & \operatorname{Im}\left\{d_{D}\right\} \\ \Delta h_{H} & \Delta h_{D} & \Delta d_{H} & \Delta d_{D} & \operatorname{Re}\left\{z_{H}\right\} & \operatorname{Im}\left\{z_{H}\right\} & \operatorname{Re}\left\{z_{D}\right\} & \operatorname{Im}\left\{z_{D}\right\} \\ \Delta z_{H} & \Delta z_{D} & R_{x} & R_{y} & R_{z} & & & \end{array}$




\section{A.5 Verwendete Programme}

\section{A.5.1 Datenerfassung und Prozessing}

Das Lesen der Daten aus dem statischen Speicherblock des RAP-Loggers und die Weiterverarbeitung in das binäre Speicherformat *. goe erfolgte mit den Programmen

- ramtra [STEVELING]

- congoe [STEVELING].

Zur Verarbeitung der Zeitreihen und Bestimmung der Übertragungsfunktionen wurden die Programme

- dezidezi [EISEL, BAHR, STEVELING]

- select [GU, BAHR]

- analys [BAHR]

- spetra [EISEL, BAHR]

verwendet.

\section{A.5.2 Berechnung der Darstellungsgrößen mit emdisp}

Die Berechnung sämtlicher Darstellungsgrößen aus den Übertragungsfunktionen $\underline{\mathbf{Z}}$ und $\underline{\mathbf{W}}$ geschah mit dem Programm emdisp, welches im Rahmen dieser Arbeit erstellt wurde.

Damit ist es möglich, die mtt- oder gdt-Files beliebig vieler Stationen inklusive Koordinaten einzulesen und gleichzeitig zu verarbeiten. Das Programm gestattet dann die Berechnung verschiedener Darstellungsgrößen, die aus den eingelesenen Übertragungsfunktionen abgeleitet werden. Dies sind unter anderem:

\section{Magnetotellurik}

- Scheinbarer spezifischer Widerstand $\rho_{a}$

- Phase der Impedanz $\phi$

- $\rho^{*}\left(z^{*}\right)$ 
- Lokale Induktionspfeile

- Rotationswinkel und Schiefe nach BAHR und SWIFT sowie Winkel der maximalen Phasenaufspaltung

- Rotationsinvariante von $\underline{\mathbf{Z}}$

\section{Erdmagnetische Tiefensondierung}

- Magnetische Übertragungsfunktionen

- Perturbationspfeile

- Rotationsinvariante von $\underline{\mathbf{W}}$

Eine Rotation sämtlicher Übertragungsfunktionen ist möglich. Die Ausgabe erfolgt in Form von einfachen Tabellen, die sowohl eine Darstellung der Parameter in Abhängigkeit der Periode erlauben, als auch eine flächenhafte Auftragung gestatten. 



\section{DANKSAGUNG}

An dieser Stelle möchte ich allen danken, die mich bei der Anfertigung dieser Arbeit unterstützt und zu ihrem Gelingen beigetragen haben.

Mein Dank gehört vor allem Herrn Prof. Dr. Karsten Bahr, der die Arbeit betreut hat und ihre Entstehung immer mit wertvollen Hinweisen und fachlicher Unterstützung begleitet hat. Er stellte während ihrer Anfertigung meine Finanzierung durch eine Stelle als wissenschaftlicher Angestellter sicher.

Herrn Prof. Dr. Ulrich Christensen danke ich für die Übernahme des Korreferats.

Wolfgang Soyer, Oliver Kuras und Mark Hönig (,MT-Team“) bin ich für die erstklassige Zusammenarbeit während der gesamten Meßkampagne sehr dankbar. Die anregenden Gespräche waren immer hilfreich, und die Zeit außerhalb aller fachlichen Diskussionen hat viel Spaß gemacht.

Alexander Gatzemeier und Mark Hönig danke ich für die kritische Durchsicht des Manuskriptes.

Allen Mitarbeiterinnen und Mitarbeitern des Instituts für Geophysik sei für die vielseitige Unterstützung gedankt. Vor allem sind hier Herr Dr. Erich Steveling und Herr Wilfried Steinhoff zu nennen. Sie haben mich bei auftretenden Schwierigkeiten hinsichtlich der Meßgeräte und bei der Durchführung und Organisation der Meßkampagne immer tatkräftig unterstützt. Frau Erika Eggert-Heise danke ich recht herzlich für ihre Hilfsbereitschaft bei der Bewältigung von verwaltungstechnischen Problemen aller Art.

Desweiteren bin ich Herrn Hans-Jürgen Cruse, Herrn Rudolph Deppe, Herrn Rainer Hennings, Herrn Dr. Martin Leven, Herrn Jörg Schliebe und Herrn Dr. Peter Spitta für ihre Mithilfe sehr dankbar. Christiane Jantos, Gunnar Jahnke, Christian Auth und Edgar Schneider danke ich für die Mithilfe bei den Messungen.

Herrn Manfred Herden danke ich ganz besonders für seine Hilfsbereitschaft, wenn es darum ging, nicht fachspezifische Probleme in den Griff zu bekommen.

Der „Kellerbelegschaft“ Cristiane Jantos, Alexander Gatzemeier und Edgar Schneider möchte ich für die vielen unterhalsamen Stunden während meiner Arbeit sehr danken.

Allen Forstbeamten der Eifel und Umgebung danke ich für ihre freundliche Unterstützung. Vielen Dank an die Mitarbeiterinnen und Mitarbeiter der FU Berlin, des GFZ Potsdam und der FU Frankfurt, die bei den Messungen mitgeholfen haben.

Besonders dankbar bin ich Herrn Prof. Dr. Ulrich Schmucker, mit dem ich viele Diskussionen geführt habe, die nicht nur in fachlicher Hinsicht wertvoll waren.

Abschließend möchte ich mich bei meinen Eltern bedanken, die mir das Studium ermöglicht und mich fortwährend unterstützt haben. Darüberhinaus ein dickes Dankeschön für dies und das an meine Schwester Nicole. Meinem Vater danke ich außerdem für die Mithilfe bei der Meßkampagne.

$\mathrm{Zu}$ guter Letzt möchte ich ganz besonders Andrea danken, die mir immer hilfreich zur Seite stand und mir viele Hinweise beim Formulieren der Arbeit gab. 



\section{LEBENSLAUF}

Jörg Leibecker

geb. am 12.5.1970 in Osnabrück

Seit 1.7.1997

1.11 .1996

7.4.1993

5.4 .1993

24.9.90

$1989-1990$

Wissenschaftlicher Angestellter

am Institut für Geophysik

der Universität Göttingen

Diplomprüfung

Titel der Diplomarbeit:

„Elektromagnetische Induktion durch Längstwellensender -

Untersuchung einer geologischen Struktur und Entwicklung

eines Verfahrens zur Bestimmung der tensoriellen Impedanz"

Immatrikulation an der

Universität Göttingen, Fach Physik

Diplomvorprüfung

Immatrikulation an der

Universität Osnabrück, Fach Physik

10.5.1989

$1982-1989$

Wehrdienst

$1980-1982$

$1976-1980$

Abitur

Gymnasium Bramsche

Orientierungsstufe Rulle

Grundschule Wallenhorst 

Natürlich gibt es eine jenseitige Welt. Die Frage ist nur: wie weit ist sie von der Innenstadt entfernt, und wie lange hat sie offen?

Woody Allen 